\title{
A Discrete Variable Representation for Electron-Hydrogen Atom Scattering
}

\author{
Lionel Francis Gaucher \\ Ph.D. Thesis \\ Department of Chemistry \\ University of California
}

and

Chemical Sciences Division Lawrence Berkeley Laboratory

University of California

Berkeley, California 94720

August 1994

This work was supported in part by the Director, Office of Energy Research, Office of Basic Energy Sciences, U. D. Department of Energy under Contract No. DE-AC03-76SF00098, Chemical Sciences Division of the U.S. Department of Energy under Contract
and in part by the National Science Foundation. 


\section{DISCLAIMER}

This report was prepared as an account of work sponsored by an agency of the United States Government. Neither the United States Government nor any agency thereof, nor any of their employees, make any warranty, express or implied, or assumes any legal liability or responsibility for the accuracy, completeness, or usefulness of any information, apparatus, product, or process disclosed, or represents that its use would not infringe privately owned rights. Reference herein to any specific commercial product, process, or service by trade name, trademark, manufacturer, or otherwise does not necessarily constitute or imply its endorsement, recommendation, or favoring by the United States Government or any agency thereof. The views and opinions of authors expressed herein do not necessarily state or reflect those of the United States Government or any agency thereof. 


\section{A Discrete Variable Representation for Electron-Hydrogen Atom Scattering}

Copyright $@ 1994$

by Lionel Francis Gaucher

The U.S. Department of Energy has to right to use this thesis for any purpose whatsoever including the right to reproduce all or any part thereof 
A Discrete Variable Representation for
Electron-Hydrogen Atom Scattering

by

Lionel Francis Gaucher

S.B. (Massachusetts Institute of Technology) 1986

\author{
DISSERTATION \\ Submitted in partial satisfaction of the requirements for the degree of \\ DOCTOR OF PHILOSOPHY \\ in \\ CHEMISTRY \\ in the \\ GRADUATE DIVISION \\ of the \\ UNIVERSITY of CALIFORNIA at BERKELEY
}

Committee in charge:

\author{
Professor William H. Miller, Chair \\ Professor William A. Lester, Jr. \\ Professor Charles L. Schwartz
}


Abstract

\title{
A Discrete Variable Representation for Electron-Hydrogen Atom Scattering
}

by

\author{
Lionel Francis Gaucher \\ Doctor of Philosophy in Chemistry \\ University of California at Berkeley \\ Professor William H. Miller, Chair
}

A discrete variable representation (DVR) suitable for treating the quantum scattering of a low energy electron from a hydrogen atom is presented. The benefits of DVR techniques (e.g. the removal of the requirement of calculating multidimensional potential energy matrix elements and the availability of iterative sparse matrix diagonalization/inversion algorithms) have for many years been applied successfully to studies of quantum molecular scattering. Unfortunately, the presence of a Coulomb singularity at the electrically unshielded center of a hydrogen atom requires high radial grid point densities in this region of the scattering coordinate, while the presence of finite kinetic energy in the asymptotic scattering electron also requires a sufficiently large radial grid point density at moderate distances from the nucleus. The constraints imposed by these two length scales have made application of current DVR methods to this scattering event difficult.

Chapter 2 of this thesis is a short aside demonstrating the superiority of the $S$ matrix formulation of the Kohn variational principle over the Schwinger variational principle for elastic S-wave scattering of an electron from a hydrogen atom within the static exchange approximation. It is shown that while the Schwinger principle suffers from the same spurious non-physical singularities usually associated with the 
K matrix formulation of the Kohn principle, the $\mathrm{S}$ matrix formulation demonstrates no such defect.

Chapter 3 introduces the main work of this thesis. A DVR for low energy electron-hydrogen atom scattering is developed which creates a radial grid point density well-suited to the two length scale constraints previously mentioned. This is accomplished through the diagonalization of the position operator within a truncated basis set of reference potential eigenstates. The reference potential chosen is the effective local potential for scattering from a static hydrogen $\phi_{2 s}$ state since this demonstrates properly-scaled Coulomb behavior at the atomic nucleus and Yukawa behavior asymptotically. The multi-dimensional DVR is composed of a direct product of two such radial DVRs and one anguiar Gauss-Legendre DVR, partitioned into the proper irreducible group representations.

Chapter 4 combines this composite DVR in the $S$ matrix formulation of the Kohn variational principle to calculate $S$ matrix elements and eigenphase sums for electron-hydrogen atom scattering for $J_{\text {total }}=0$ between the $n=2$ and $n=3$ thresholds. Results are compared to those of other theoretical methods.

Chapter 5 proposes an important improvement in the technique. While the condition number of the multi-dimensional Hamiltonian is found to be too large to permit application of low-memory iterative matrix inversion technology, a method is proposed which greatly reduces the condition number of the Hamiltonian by effectively filtering out high energy elements of the $L^{2}$ basis.

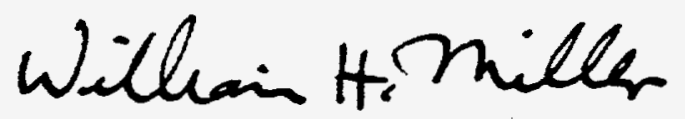




\section{Contents}

List of Figures $\quad \mathbf{v}$

List of Tables $\quad$ vii

Acknowledgements viii

1 Introduction 1

1.1 Classic Studies of $e^{-}+H$ Scattering . . . . . . . . . . . 1

1.2 Discrete Variable Representations ................ . . 3

1.3 Outline ........................ 7

2 On the Absence of Anomalous Singularities... 9

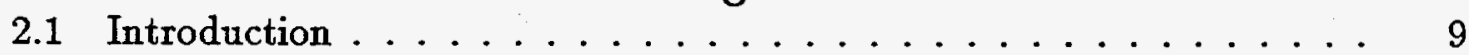

2.2 S Matrix Kohn Variational Principle .............. 11

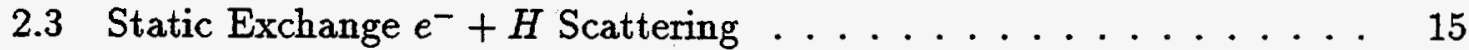

2.4 Results and Discussion .................. 16

3 The Theory 22

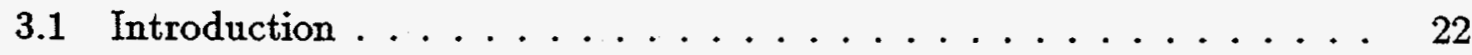

3.2 Hamiltonian and Coordinate System . . . . . . . . . . . . 23

3.3 Review of S Matrix Kohn Variational Principle . . . . . . . . 24

3.4 DVR for the Interaction Region . . . . . . . . . . . . . . . . 26

3.4.1 Necessary Characteristics . . . . . . . . . . . . . 26

3.4.2 Sturmian Functions and Potential Optimized DVR . . . . . 27

3.4.3 Radial DVR for Screened Coulomb Potentials . . . . . . . . . 29

3.4.4 Gauss-Legendre DVR for Angular Coordinate . . . . . . . . 40

3.5 Form of the Symmetrized Hamiltonian . . . . . . . . . . . . 41

3.5.1 Benefits of Symmetrization . . . . . . . . . . . . . 41

3.5.2 Calculation of the Hamiltonian Matrix Elements . . . . . . 43

3.6 Representation of the Free Functions . . . . . . . . . . . . 45

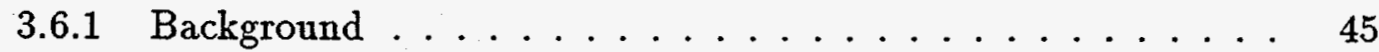

3.6.2 Derivation of the Free Functions . . . . . . . . . . 50 
3.7 Calculation of the Bound-Free Matrix Elements . . . . . . . . . 64

3.7.1 The Extended Radial DVR ................... 67

3.7.2 Symmetrization of the Free Functions . . . . . . . . . . 69

3.8 Calculation of the Free-Free Matrix Elements . . . . . . . . 70

4 Results and Discussion $\quad 73$

4.1 Setting Parameter Values ......................... 73

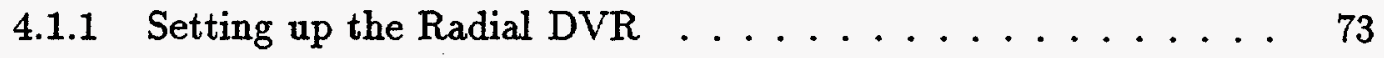

4.1.2 Setting up the Free Function Expansion . . . . . . . . . 79

4.2 Singlet and Triplet Scattering Calculations . . . . . . . . . . . 84

4.2.1 Convergence of Singlet Transition Probabilities . . . . . . . 85

4.2 .2 Convergence of Singlet S Matrices . . . . . . . . . . . . . . . 104

4.2.3 Transition Probabilities and S Matrices . . . . . . . . . . . 114

4.2 .4 Cross Sections . . . . . . . . . . . . . . . . . 129

4.2.5 "Distinguishable" Electrons ............... . . 132

4.3 Comparisons with other Theoretical Results . . . . . . . . . 135

4.3.1 Cross Section Comparisons . . . . . . . . . . . 135

4.3.2 Eigenphase Sum Comparisons ............. 138

5 Improvements $\quad 142$

$\begin{array}{ll}\text { Bibliography } & \mathbf{1 4 7}\end{array}$ 


\section{List of Figures}

$2.1 \tan \delta$ as a function of $\alpha$ for singlet $(S=0)$ scattering at $k=1$ au $\ldots \quad 17$

2.2 Same quantity as in previous figure for a wider $\alpha$ range $\ldots \ldots \ldots 18$

$2.3 \tan \delta$ as a function of $k$ for singlet $(S=0)$ scattering with $\alpha=0.94 \quad, \quad 19$

2.4 Same quantity as in previous figure but with $\alpha=4.0 \ldots \ldots 20$

$2.5 \tan \delta$ as a function of $k$ for triplet $(S=1)$ scattering with $\alpha=0.5 \ldots 21$

3.1 Effective potential for $e^{-}$scattering from a static $\phi_{1 s}$ or $\phi_{2 s} \mathrm{H}$ state . 32

$3.2 \operatorname{Re}\left\langle\phi_{1 s}|\hat{H}-\hat{E}| \Psi_{0(2 s)}\right\rangle$ for $E=0.78 \operatorname{Ryd} \ldots \ldots \ldots \ldots \ldots$

$3.3 \operatorname{Re}\left\{\phi_{2 s}|\hat{H}-\hat{E}| \Psi_{0(2 s)}\right\rangle$ for $E=0.78 \operatorname{Ryd} \ldots \ldots \ldots \ldots \ldots$

$3.4 \operatorname{Re}\left\langle\phi_{2 p}|\hat{H}-\hat{E}| \Psi_{0(2 s)}\right\rangle$ for $E=0.78 \operatorname{Ryd} \ldots \ldots \ldots \ldots \ldots . \ldots \ldots$

$3.5 \operatorname{Re}\left\langle\phi_{1 s}|\hat{H}-\hat{E}| \Psi_{0(2 s)}\right\rangle$ for $E=0.82 \operatorname{Ryd} \ldots \ldots \ldots \ldots \ldots \ldots$

$3.6 \operatorname{Re}\left\langle\phi_{2 s}|\hat{H}-\hat{E}| \Psi_{0(2 s)}\right\rangle$ for $E=0.82 \operatorname{Ryd} \ldots \ldots \ldots \ldots \ldots$

$3.7 \operatorname{Re}\left\langle\phi_{2 p}|\hat{H}-\hat{E}| \Psi_{0(2 s)}\right\rangle$ for $E=0.82 \operatorname{Ryd} \ldots \ldots \ldots \ldots \ldots$

$3.8 \operatorname{Re}\left\langle\phi_{1 s}|\hat{H}-\hat{E}| \Psi_{0(2 s)}\right\rangle$ for $E=0.88 \operatorname{Ryd} \ldots \ldots \ldots \ldots \ldots \ldots$

$3.9 \operatorname{Re}\left\langle\phi_{2 s}|\hat{H}-\hat{E}| \Psi_{0(2 s)}\right\rangle$ for $E=0.88 \operatorname{Ryd} \ldots \ldots \ldots \ldots \ldots$

$3.10 \operatorname{Re}\left\langle\phi_{2 p}|\hat{H}-\hat{E}| \Psi_{0(2 s)}\right\rangle$ for $E=0.88 \operatorname{Ryd} \ldots \ldots \ldots \ldots \ldots$

3.11 Mixing of $H$ bound states for asymptotic $\phi_{2 s}$ state . . . . . . . 59

3.12 Mixing of $\mathrm{H}$ bound states for Asymptotic $\phi_{2 p}$ state $\ldots \ldots \ldots 60$

4.1 Radial DVR distributions . . . . . . . . . . . . 77

4.2 Radial DVR distributions within the interaction region . . . . . 78

4.3 Radial DVR function toward the end of the interaction region . . . 79

4.4 Radial DVR function within the interaction region . . . . . . . 80

4.5 Radial DVR function toward the end of the extended DVR region . . 81

4.6 Cutoff function $f$ in comparison to $H$ bound states . . . . . . 82

4.7 Projection of $F_{\mathbf{n} i}$ for $\mathbf{n}=1$ and $i=1$ for $E=0.88 \mathrm{Ryd}$ ( $30 \hat{x}$ eigenstates) 83

4.8 Projection of $F_{\mathbf{n} i}$ for $\mathbf{n}=1$ and $i=1$ for $E=0.88 \mathrm{Ryd}$ ( $35 \hat{x}$ eigenstates) 84

4.9 Projection of $F_{\mathbf{n} i}$ for $\mathbf{n}=1$ and $i=1$ for $E=0.88$ Ryd ( $40 \hat{x}$ eigenstates) 85

4.10 Projection of $F_{\mathbf{n} i}$ for $\mathbf{n}=1$ and $i=1$ for $E=0.88$ Ryd (45 $\hat{x}$ eigenstates) 86

$4.11 \alpha$ convergence of $\phi_{1 s} \rightarrow \phi_{1 s}$ transition probability $\ldots \ldots \ldots 88$

$4.12 \alpha$ convergence of $\phi_{1 s} \rightarrow \phi_{2 s}$ transition probability $\ldots \ldots \ldots . \ldots 89$ 
$4.13 \alpha$ convergence of $\phi_{1 s} \rightarrow \phi_{2 p}$ transition probability . . . . . . . 89

$4.14 \alpha$ convergence of $\phi_{2 s} \rightarrow \phi_{2 s}$ transition probability . . . . . . . 90

$4.15 \alpha$ convergence of $\phi_{2 s} \rightarrow \phi_{2 p}$ transition probability . . . . . . . . 90

$4.16 \alpha$ convergence of $\phi_{2 p} \rightarrow \phi_{2 p}$ transition probability ......... 91

$4.17 n_{\gamma}$ convergence of $\phi_{1 s} \rightarrow \phi_{1 s}$ transition probability . . . . . . . . 93

$4.18 n_{\gamma}$ convergence of $\phi_{1 s} \rightarrow \phi_{2 s}$ transition probability . . . . . . . . . 93

$4.19 n_{\gamma}$ convergence of $\phi_{1 s} \rightarrow \phi_{2 p}$ transition probability ......... 94

$4.20 n_{\gamma}$ convergence of $\phi_{2 s} \rightarrow \phi_{2 s}$ transition probability ......... 94

$4.21 n_{\gamma}$ convergence of $\phi_{2 s} \rightarrow \phi_{2 p}$ transition probability ......... 95

$4.22 n_{\gamma}$ convergence of $\phi_{2 p} \rightarrow \phi_{2 p}$ transition probability . . . . . . . . 95

$4.23 R_{\max }$ convergence of $\phi_{1 s} \rightarrow \phi_{1 s}$ transition probability ....... 96

$4.24 R_{\max }$ convergence of $\phi_{1 s} \rightarrow \phi_{2 s}$ transition probability . . . . . . 97

$4.25 R_{\max }$ convergence of $\phi_{1 s} \rightarrow \phi_{2 p}$ transition probability . . . . . . 97

$4.26 R_{\max }$ convergence of $\phi_{2 s} \rightarrow \phi_{2 s}$ transition probability . . . . . . 98

$4.27 R_{\max }$ convergence of $\phi_{2 s} \rightarrow \phi_{2 p}$ transition probability . . . . . . 98

$4.28 R_{\max }$ convergence of $\phi_{2 p} \rightarrow \phi_{2 p}$ transition probability . . . . . . 99

$4.29 p_{\max }$ convergence of $\phi_{1 s} \rightarrow \phi_{1 s}$ transition probability . . . . . . . 101

$4.30 p_{\max }$ convergence of $\phi_{1 s} \rightarrow \phi_{2 s}$ transition probability . . . . . . . 101

$4.31 p_{\max }$ convergence of $\phi_{1 s} \rightarrow \phi_{2 p}$ transition probability . . . . . . . . 102

$4.32 p_{\max }$ convergence of $\phi_{2 s} \rightarrow \phi_{2 s}$ transition probability . . . . . . . . 102

$4.33 p_{\max }$ convergence of $\phi_{2 s} \rightarrow \phi_{2 p}$ transition probability . . . . . . . 103

$4.34 p_{\max }$ convergence of $\phi_{2 p} \rightarrow \phi_{2 p}$ transition probability . . . . . . . . 103

$4.35 n_{R}^{e}$ convergence of $\phi_{1 s} \rightarrow \phi_{1 s}$ transition probability . . . . . . . 105

$4.36 n_{R}^{e}$ convergence of $\phi_{1 s} \rightarrow \phi_{2 s}$ transition probability . . . . . . . 105

$4.37 n_{R}^{e}$ convergence of $\phi_{1 s} \rightarrow \phi_{2 p}$ transition probability . . . . . . 106

$4.38 n_{R}^{e}$ convergence of $\phi_{2 s} \rightarrow \phi_{2 s}$ transition probability . . . . . . . . 106

$4.39 n_{R}^{e}$ convergence of $\phi_{2 s} \rightarrow \phi_{2 p}$ transition probability . . . . . . . . 107

$4.40 n_{R}^{e}$ convergence of $\phi_{2 p} \rightarrow \phi_{2 p}$ transition probability . . . . . . . 107

4.41 Converged transition probabilities for singlet scattering from $\phi_{1 s}$ state 124

4.42 Converged transition probabilities for singlet scattering from $\phi_{2 s}$ state 125

4.43 Converged transition probabilities for singlet scattering from $\phi_{2 p}$ state 125

4.44 Converged transition probabilities for triplet scattering from $\phi_{2 s}$ state 130

4.45 Converged transition probabilities for triplet scattering from $\phi_{2 p}$ state 131

4.46 Elastic cross section $\sigma_{1 s \rightarrow 1 s} \ldots \ldots \ldots \ldots \ldots 131$

4.47 Inelastic cross sections $\sigma_{1 s \rightarrow 2 s}$ and $\sigma_{1 s \rightarrow 2 p} \ldots \ldots \ldots \ldots$. . . . . . . 132

4.48 Direct and exchange transition probabilities $P_{11}^{\text {dir }}$ and $P_{11}^{e x} \ldots \ldots$. . . 133

4.49 Direct and exchange transition probabilities $P_{22}^{\text {dir }}$ and $P_{22}^{e x} \ldots \ldots . . .134$

4.50 Direct and exchange transition probabilities $P_{23}^{d i r}$ and $P_{23}^{e x} \ldots \ldots$. . . 134

4.51 Direct and exchange transition probabilities $P_{33}^{\text {dir }}$ and $P_{33}^{\text {ex }} \ldots \ldots$

5.1 Effect on $\mathrm{V}$ of transforming angular DVR to FBR . . . . . . . 145 


\section{List of Tables}

4.1 Convergence of singlet $\operatorname{Re}\left(\mathbf{S} \cdot \mathbf{S}^{\dagger}\right)_{11}$ with respect to $\alpha \ldots \ldots$. . . . . 109

$4.2 R_{\max }$ convergence of singlet $S_{1 x}$ matrix elements at lower kinetic energies 111

4.3 $R_{\max }$ convergence of singlet $S_{1 x}$ matrix elements at medium kinetic energies .......................... 112

4.4 $R_{\max }$ convergence of singlet $S_{1 x}$ matrix elements at higher kinetic energies 113

4.5 Converged S matrix elements for singlet scattering . . . . . . . . . 115

4.6 Converged S matrix elements for singlet scattering . . . . . . . . . . . 116

4.7 Converged S matrix elements for singlet scattering . . . . . . . . . . 117

4.8 Converged S matrix elements for singlet scattering . . . . . . . . . 118

4.9 Converged S matrix elements for singlet scattering . . . . . . . . . 119

4.10 Converged S matrix elements for singlet scattering . . . . . . . . . . 120

4.11 Converged S matrix elements for singlet scattering . . . . . . . . . . . 121

4.12 Converged S matrix elements for singlet scattering . . . . . . . . . . 122

4.13 Converged S matrix elements for singlet scattering . . . . . . . . . . 123

4.14 Converged S matrix elements for triplet scattering . . . . . . . . . . 126

4.15 Converged S matrix elements for triplet scattering . . . . . . . . . . 127

4.16 Converged S matrix elements for triplet scattering . . . . . . . . . 128

4.17 Comparison of singlet excitation cross sections . . . . . . . . . . . 136

4.18 Comparison of singlet eigenphase sums . . . . . . . . . . . . . . . . . . . . . . . . . . . . .

4.19 Comparison of triplet eigenphase sums ............. . . 141 
viii

\section{Acknowledgements}

An endeavor such as this does not come to fruition without a great deal of assistance from many people in many different ways. I would first like to thank my research director Professor William $H$. Miller for providing the intellectual guidance and financial support which made this work possible, and for allowing me the scientific freedom to explore a variety of ideas. His patience and understanding made success in a challenging research project attainable. I would also like to thank Cheryn Gliebe for her administrative skill in keeping the wheels of the Miller Group rolling, and for her friendship.

The graduate students and postdoctoral fellows who have worked in the Miller Group during the last several years have been a great scientific and social resource for me. The Group's unique mix of intelligence, diligence, humor, and interesting personalities has made it a special environment in which to work. And it is only now that I am leaving that I realize how lucky I have been to have shared this time with them. I would like to specifically thank Professor Nancy Makri for introducing me to the Group. Professor John Zhang provided me with much needed guidance during my first research project. Dan Gezelter and Ward Thompson are to be commended for their computer system administration work. Dr. Gerrit Groenenboom was a source of great scientific insight during many lengthy conversations, as was Dr. Scott Auerbach. Dr. Rigoberto Hernandez provided valuable assistance in using $\operatorname{IAT}_{\mathrm{E}} \mathrm{X}$ to format this thesis.

I would be remiss if I were to not single out for special praise Professor Claude Leforestier of Laboratoire de Chimie Théorique, Université de Paris-Sud. His sabbatical leave spent here at the University of California was a tremendously positive 
influence on my research effort. And his considerable knowledge, energy, and concern have made a lasting impression. I will always be in his debt.

My friendships with certain members of the "old guard" of my incoming class have continued to endure. John Gehlen and Art Mahoney have been my closest friends and confidants during this program, and they continue to be so. And I have always regretted Andrew Bennett's early departure from the program, as he was one of the most colorful people to grace the theory floor during my stay here. Barb Bryan has been a frequent and welcome voice of reason. Kevin Leung and I have had a special bond that only hopelessly nocturnal graduate students can share. (Fortunately Bruce Spath will remain behind to carry on the tradition.) And Dr. Jonathan Wise has been an excellent friend and kindred spirit over the years, despite our recent separation by hundreds of miles. Such friendships are worth their weight in lead, as I'm sure he would agree.

Many of my old friendships from my undergraduate days at MIT have survived and thrived here in California. Denis Gulsen, Chris Racicot, Andy Brockman, and Dan Mittleman have been loyal friends. They have provided a welcome relief from the pressures of graduate school (as well as an occasional foosball game).

Martin Isaacson and Srihari Keshavamurthy have thankfully proven that some people can tolerate living with me for an extended period of time and Luis Porcelli was arguably the best neighbor I've ever had. Drs. Johanna Weber, Allison Sekuler, Jim Crowell, and Lisa Shannon have been a great source of entertainment over the years. Johanna, in particular, has been a good friend during occasionally trying times. And George Atkins has been...well...unique. Nick and Dr. Mindy Lai have been generous friends and have far too much class for their own good. They've allowed me to see graduate school from a different perspective. Barbara Lee has been much more than a close friend and confidante. And Aimee Powell has meant more to me than she will ever know or admit.

I must offer special thanks to my Calculus teacher at Saint John High School, Mr. Stephen Gregory. He took great interest in me as a student and selflessly showed me a path to academic achievement. His encouragement has greatly influenced my life. 
Finally, and most importantly, I would like to thank my parents, Ronald and Phyllis Gaucher. Their love, encouragement, and support have given me the strength to push forward during difficult and trying times. They, more than any others, have experienced my joys and pains with me. This thesis is therefore dedicated with my love to them.

This work was supported under the Director, Office of Energy Research, Office of Basic Energy Sciences, Chemical Sciences Division of the U. S. Department of Energy under Contract No. DE-AC03-76SF00098, and also by the National Science Foundation under Grant No. CHE-8920690. 


\section{Chapter 1}

\section{Introduction}

\subsection{Classic Studies of $e^{-}+H$ Scattering}

The development of the theory of quantum mechanics ushered in a new era in the study of basic physical phenomena, and the theory of scattering processes has been influenced greatly by it. Extensive reviews can be found in Wu and Ohmura [1], Mott and Massey [2], Newton [3], Geltman [4], Smith [5], Joachain [6], and Taylor [7].

It is not surprising that a quantum mechanical treatment of scattering phenomena would find its first applications in the most basic of scattering processes. Due to its high degree of symmetry and simplicity, the scattering of a low energy electron from a hydrogen atom is obviously in this category. Consequently, a tremendous amount of theoretical study has been brought to bear on this most simple of atomic scattering events in an effort to test various theoretical methods.

An initial approach to this problem usually begins with the so-called coupledchannel expansion [8], in which the total two-electron wavefunction is expanded in a properly symmetrized sum of projections onto various hydrogen bound states to give

$$
\Psi\left(R_{1}, R_{2}, \gamma\right)=\sum_{i} \hat{\mathcal{A}} F_{i}\left(R_{1}\right) \phi_{i}\left(R_{2}, \gamma\right)
$$

where the operator $\hat{\mathcal{A}}$ provides the proper symmetrization. (A concise review of this expansion is given by Lester [9].) Unfortunately, completeness in such an expansion is 
not approached without inclusion of a non-denumerable set of continuum functions, and proper treatment of the polarizability of the hydrogen atom becomes very difficult [10]. This renders ineffective expansions which are restricted to physical eigenstates, necessitating the inclusion of so-called pseudo-states. These can be thought of as closed-channel virtual states which exist in the continuum, and they are often derived by diagonalization of the physically correct Hamiltonian for the system within a large set of $L^{2}$ functions. The resulting coupled differential equations (which become integro-differential equations after the proper symmetrization) are then solved through a numerical integration algorithm in which proper boundary conditions are imposed on the total spatial wavefunction. An excellent review of early use of this class of methods is provided by Burke and Smith [11].

Unfortunately, the solution of the integro-differential equations through the use of these numerical integration methods has proven to be a difficult and complicated undertaking. Not surprisingly, instabilities are encountered during the numerical propagation procedure which require special treatment. And when electron exchange is included in the total wavefunction, the integro-differential equations must be solved iteratively until self-consistency is achieved.

Variational basis set methods have proven to be extremely useful alternatives to numerical integration algorithms, and excellent reviews of these methods as applied to electron-atom scattering are provided by Callaway [12] and Nesbet [13]. Several different variational methods exist, but all are based on the existence of a functional which is stationary with respect to arbitrary variations in the wavefunction.

One of the oldest variational principles associated with quantum scattering theory is the Schwinger variational principle [14] which is based on the LippmannSchwinger integral equation [15]. This formalism has been used extensively to study electron-atom scattering phenomena, and has also been applied to electron-molecule scattering (e.g. Lima et al. [16]). Unfortunately, despite the fact that the Schwinger principle naturally incorporates the proper scattering boundary conditions into the calculation, this variational principle requires the expensive calculation of matrix elements of the scattering Green's function $G_{0}$ between pairs of $L^{2}$ basis functions.

The Kohn variational principle [17] provides an alternative to the Schwinger 
principle. And although it does require the calculation of a small number of energydependent integrals, it only requires integrals of the Hamiltonian rather than of the scattering Green's function. This variational principle combined with $\mathrm{K}$ matrix boundary conditions provided the basis for an initial application of the method to elastic S-wave electron-hydrogen scattering by Schwartz [18]. Schwartz used twoelectron $L^{2}$ functions of the type employed by Hylleraas [19] in previously performed bound state calculations:

$$
\chi=\frac{1}{4 \pi \sqrt{2}} \sum_{l, m, n \geq 0} C_{l m n} e^{-(\kappa / 2)\left(R_{1}+R_{2}\right)} R_{12}^{l}\left(R_{1}^{m} R_{2}^{n} \pm R_{1}^{n} R_{2}^{m}\right) .
$$

Schwartz found that one of the defects of the Kohn variational principle was the presence of spurious singularities in the calculation of phase shifts as a function of the non-linear scale parameter $\kappa$. Analysis of the origin of these anomalous singularities led to the development of several alternative Kohn formalisms which have been reviewed by Truhlar et al. [20] and Callaway [12]. The various incarnations of the Kohn principle, together with a wide range of various pseudo-state $L^{2}$ basis sets, have been widely applied in the study of electron-hydrogen scattering as well as electron scattering from multi-electron atoms [21]. Kohn calculations have also been performed on electron-molecule systems with success [22], and the development by Miller $[23,24]$ of a Kohn formulation with complex boundary conditions has provided a method free of the anomalous singular behavior found in the $\mathrm{K}$ matrix formulation.

\subsection{Discrete Variable Representations}

Computational scattering theory has from its beginning been limited by the speed and memory requirements of its computations. And while modern computational technology has improved quite rapidly in both respects, the aspirations and imaginations of theoretical physicists and chemists constantly look beyond the prevailing technological limitations. The development of the discrete variable representation (DVR) has provided an extremely useful tool for extending the applicability of current technology to physical problems previously thought to be unapproachable. 
The main purpose of this thesis is the adaptation of the modern DVR to the basic study of quantum electron-atom scattering, beginning with the simplest example of low energy electron-hydrogen scattering. As will shortly be discussed, discrete variable representations have characteristics which make them very useful for this endeavor.

The genesis of DVR begins with the landmark work of Harris, Engerholm, and Gwinn [25], in which a method is presented which utilizes a quadrature approximation in the calculation of matrix elements of quantum mechanical operators. The method is simple:

1. The matrix representation of the position operator $\hat{x}$ is calculated within a particular basis set $\left\{\phi_{n}\right\}$.

2. A unitary-similarity transformation $U$ is derived within this basis which diagonalizes the position operator to give the new representation of the position operator $\mathbf{x}^{\prime}=\mathbf{U}^{-1} \cdot \mathbf{x} \cdot \mathbf{U}$.

3. This transformation defines a new basis set $\left\{\phi_{n}^{\prime}\right\}$ in which the position operator is diagonal. The potential energy matrix $V^{\prime}$ in this new representation is approximated by setting its diagonal elements to the values of the potential at the eigenvalues of the original $\mathrm{x}$ matrix: $V_{i i^{\prime}}^{\prime}=V\left(x_{i i^{\prime}}^{\prime}\right) \delta_{i i^{\prime}}$.

4. Other quantum mechanical operators $\hat{Q}$ are placed in the new representation by the same transformation: $Q^{\prime}=U^{-1} \cdot Q \cdot U$.

The approximation of the potential matrix in this new representation is most accurate when the potential function $V(x)$ can be easily represented by a short Taylor expansion in $x$, and its accuracy increases with the number of $\mathbf{x}$ eigenfunctions used.

Dickenson and Certain [26] showed that the $\mathrm{V}$ matrix elements generated by this method are equivalent to Gaussian quadrature approximations [27] when the original basis set $\left\{\phi_{n}\right\}$ is composed of properly weighted orthogonal polynomials. Formal application of these techniques to the coupled-channel equations of quantum scattering was done by Lill, Parker, and Light [28], and formalization of the gener- 
alized DVR in which the set of grid points $\left\{x_{i}\right\}$ can be chosen independent of the original basis set $\left\{\phi_{n}\right\}$ was performed by Light, Hamilton, and Lill [29].

The benefits of the DVR over a more conventional variational basis representation (VBR) utilizing standard basis functions became clear for multi-dimensional problems. Instead of a direct product of sets of spatially-diffuse basis functions, the multi-dimensional basis set could be represented by a direct product of grid point basis sets. The calculation of potential matrix elements was now reduced to the calculation of function values at points in multi-dimensional space, rather than expensive multi-dimensional integrations. The DVR provided a potential matrix $V$ which was diagonal.

But another great benefit is realized for Hamiltonian matrices $\mathbf{H}$ which are highly separable in the individual spatial coordinates. The resulting Hamiltonian in the DVR is sparse. This is due entirely to the fact that the potential matrix $V$ in the DVR is diagonal. For an ordinary spatially-diffuse basis set representation, the multi-dimensional kinetic energy matrix $T$ is usually sparse due to the separability of the operator.

$$
T_{i_{1} i_{2} i_{2} \ldots i_{1}^{\prime} i_{2}^{\prime} i_{3}^{\prime} \ldots}=t_{i_{1} i_{1}^{\prime}} \delta_{i_{2} i_{2}^{2}} \delta_{i_{3} i_{3}^{\prime}} \ldots+t_{i_{2} i_{2}^{\prime}} \delta_{i_{1} i_{1}^{\prime}} \delta_{i_{3} i_{3}^{\prime}} \ldots+\ldots
$$

But the potential energy matrix $\mathrm{V}$ in a conventional basis is usually not sparse at all. It is full, and therefore the entire Hamiltonian $H$ is full. This means that not only must expensive multi-dimensional integrations be performed, but the entire matrix must be stored in order to calculate its eigenvalues or to perform inversion of the Hamiltonian (or some function of the Hamiltonian). This is a tremendous liability for multi-dimensional problems since the storage requirements of the Hamiltonian grow very quickly as its dimensionality increases. But usually the complete storage of the Hamiltonian is not necessary in the DVR. One need only store the values of the potential at the composite grid points and the values of the one-dimensional matrices making up the remainder of the Hamiltonian (kinetic energy and centrifugal barrier terms).

The development of eigenvalue and matrix inversion algorithms specifically designed for sparse matrices has enabled performance of these operations on extremely 
large matrices which demand far too much memory to permit complete storage. This offers an attractive alternative to decomposition/Gaussian elimination algorithms. For example, the algorithms developed by Lanczos [30, 31, 32] merely require a subroutine which multiplies the particular matrix $\mathbf{A}$ being inverted or diagonalized by an input column vector:

$$
\mathbf{y}=\mathbf{A} \cdot \mathbf{x} .
$$

The algorithm generates solution vectors (or eigenvectors in the case of a diagonalization) within a subspace called the Krylov space [33] defined by

$$
\mathcal{K}^{N}=\left\{\mathbf{x}_{\mathbf{0}}, \mathbf{A} \mathbf{x}_{0}, \mathbf{A}^{2} \mathbf{x}_{0}, \ldots, \mathbf{A}^{N} \mathbf{x}_{0}\right\}
$$

in which $\mathbf{x}_{0}$ is a random seed vector and $N$ is the number of $\mathbf{A}$ multiplications which have been performed during the iterative inversion (or diagonalization).

The benefit of such algorithms for sparse matrices is obvious. If the vast majority of matrix elements in the matrix $\mathbf{A}$ are zero, the operation of matrix multiplication can be made computationally inexpensive. In addition, the multiplication can be accomplished without storing the entire matrix. It is only necessary to store "bookkeeping" information which identifies non-zero matrix elements within a row of the matrix A.

The applications of discrete variable representations in the chemical literature are quite numerous. Lill, Parker, and Light [34] have presented the application of a combination of a finite basis representation (FBR) with a DVR for atom-diatom collisions. Choi and Light [35] have successfully applied DVR to the determination of bound and quasibound states of the $\mathrm{Ar}-\mathrm{HCl}$ van der Waals complex. Leforestier [36] has presented a grid representation for rotating triatomic molecules using the generalized DVR of Light, Hamilton, and Lill [29], and a DVR based on Gauss-Lobatto quadrature $[37$, p. 888] and Lagrangian interpolation polynomials was developed by Manolopoulos and Wyatt [38] for use in studying quantum reactive molecular scattering within the formulation of Miller [39]. A potential-optimized DVR representation in which the placement of the grid points is dictated by the shape of the potential energy surface can be found in the calculation of the quantum mechanical rate constant for the reaction $\mathrm{H}+\mathrm{O}_{2} \rightarrow \mathrm{OH}+\mathrm{O}$ by Leforestier and Miller [40]. 
Colbert and Miller [41] have developed an extremely general DVR which provides equally-spaced grid points (see also Schwartz [42]) and has proven useful in reactive molecular scattering [43]. Seideman and Miller [44] have computed cumulative reaction probabilities using this DVR by augmenting it with a steadily increasing complex (optical) potential in the asymptotic region of the scattering coordinate. Thompson and Miller [45] have used a similar formalism to calculate initial state-selected and state-to-state molecular reaction probabilities. A technique for reformulating the calculation of the cumulative molecular reaction probability as an eigenvalue problem using the DVR of Colbert and Miller [41] and the complex potential of Seideman and Miller [44] is presented by Manthe and Miller [46] and has been used to calculate the rate constant for the reaction $\mathrm{H}_{2}+\mathrm{OH} \rightarrow \mathrm{H}_{2} \mathrm{O}+\mathrm{H}$ [47].

Time-dependent methods which apply discrete variable representations in the direct calculation of the time-dependent wavefunction through operation of the quantum mechanical time propagator on an initial state, indicated by

$$
\psi(\mathbf{R}, t)=\exp \left(-\frac{i \hat{H} t}{\hbar}\right) \psi(\mathbf{R}, 0)
$$

are quite popular and extensively used. An excellent review is given by Kosloff [48].

\subsection{Outline}

The general subject of this thesis is the study of quantum electron-hydrogen scattering at low energies. Chapter 2 [49] is a short aside in which it is discovered that although the Schwinger variational principle has been shown by Apagyi, Lévay, and Ladányi [50] to produce spurious singularities when applied to S-wave electronhydrogen scattering within the static exchange approximation, the $\mathrm{S}$ matrix formulation [24] of the Kohn variational principle has no such singularities when applied to the same scattering system.

But the main focus of this thesis is the application of discrete variable representation methods to the problem of low energy electron-atom scattering, specifically to the simplest case of electron-hydrogen scattering for $J_{\text {total }}=0$. Unfortunately, the application of specific DVR techniques which have proven to be useful in quantum 
molecular scattering do not provide adequate treatment of quantum electron-atom scattering. Chapter 3 presents the development of a DVR for electron-atom scattering which is tailored specifically to the physics of these events and alleviates the particular mathematical problems which result from the scattering of electrons from atoms. Chapter 4 applies this theory to the quantum scattering of an electron from a hydrogen atom at energies between the $n=2$ and $n=3$ thresholds for $J_{\text {total }}=0$. Chapter 5 presents an improvement to the theory which would permit the application of low memory iterative matrix inversion algorithms to the calculation. 


\section{Chapter 2}

\section{On the Absence of Anomalous}

\section{Singularities in the $\mathbf{S}$ Matrix}

Version of the Kohn Variational

\section{Principle}

\subsection{Introduction}

One of the simplest variational principles associated with quantum scattering theory is the Kohn Variational Principle [17]. It shows a striking similarity to the Raleigh-Ritz Principle, which is commonly used in the variational calculation of eigenvalues in standard quantum mechanical bound state problems. The presence of an inhomogeneous boundary condition in the scattering problem merely introduces a surface term into the formula, so that the functional which is extremized in the Kohn principle takes the form

$$
F=\tilde{F}+\langle\tilde{\psi}|\hat{H}-\hat{E}| \tilde{\psi}\rangle
$$

where $\tilde{\psi}$ is the trial wavefunction which is extremized and satisfies the proper boundary conditions, $\hat{H}$ is the Hamiltonian for the system, and $E$ is the total energy of the system. The inhomogeneous term $\tilde{F}$ is the boundary condition term which depends 
upon which formulation of the Kohn principle is being employed.

The application of the Kohn principle in actual potential scattering is quite simple. One need merely calculate matrix elements of the Hamiltonian as a function of a particular basis set. Unfortunately, past applications of the Kohn principle with $\mathrm{K}$ matrix boundary conditions $[18,13]$ have demonstrated the presence of non-physical anomalous singularities. Oddly enough, the $S$ matrix formulation (using complex basis functions rather than the purely real sine and cosine functions) of the Kohn principle provides a mechanism by which these "Kohn anomalies" can be avoided [23]. The $S$ matrix formulation [24] has provided a useful means to solve molecular reactive scattering problems $[51,52,53,54]$ as well as electron-atom/molecule scattering problems $[55,56,22,57]$.

Another variational principle widely used in the study of scattering phenomena is the Schwinger Variational Principle [14, 58]. It has found wide application in the study of electron-atom scattering $[59,16]$. It is more difficult to apply than the Kohn principle because it requires the calculation of matrix elements of the energy-dependent operator $\hat{V} \hat{G}_{0} \hat{V}$ where $V$ is the scattering potential and $G_{0}$ is the energy-dependent Green's function for a convenient reference problem.

One of the desirable features of the Schwinger principle is that until recently it was thought that it provided an "anomaly-free" scattering calculation. But Apagyi et al. [50] have applied the Schwinger principle to the problem of S-wave electronhydrogen scattering within a static exchange approximation and found the existence of exactly such non-physical singularities when the non-local portion of the static exchange potential is included. (The anomalous singularities are not found when only the local, non-exchange portion of the potential is included.)

This has important implications for applications to quantum mechanical reactive scattering theory. The rearrangement of atoms in a reactive collision of molecules has been shown by Miller [39] to generate similar non-local exchange terms in the applicable coupled-channel equations. As such, anomalous singularites could in principle occur in these calculations too. It is the purpose of this chapter to apply the $S$ matrix version of the Kohn variational principle to the same physical system studied by Apagyi et al in an effort to determine whether this method suffers from 
the same unfortunate characteristic.

Section 2 of this chapter provides a short review of the $S$ matrix formulation of the Kohn variational principle and compares it to the $\mathrm{K}$ matrix formulation, while demonstrating why the $S$ matrix formulation is free from the aforementioned nonphysical anomalies. Section 3 presents the results of applying the $S$ matrix formulation to the S-wave static exchange approximation of $e^{-}+H$ scattering and compares these results to the results of Apagyi et al. (It will be noted that the same basis set is used in both calculations.)

\subsection{S Matrix Kohn Variational Principle}

In this section, the main points in the derivation of the $S$ matrix formulation of the Kohn variational principle are reviewed. A more detailed description is provided by Zhang, Chu, and Miller [24]. Atomic units are used throughout this section ( $\hbar=$ $\left.m_{e}=1\right)$.

In the $\mathrm{S}$ matrix formulation of the Kohn principle, the functional in (2.1) which is treated variationally is the $\mathbf{S}$ matrix itself associated with a trial wavefunction $\tilde{\psi}:$

$$
\mathbf{S}[\tilde{\psi}]=\tilde{\mathbf{S}}+i\langle\tilde{\psi}|\hat{H}-\hat{E}| \tilde{\psi}\rangle
$$

Here, $\tilde{\psi}$ is a trial function which is treated variationally and has (for the case of elastic scattering involving only one open channel) the asymptotic form

$$
\lim _{R \rightarrow+\infty} \tilde{\psi}(R)=k^{-\frac{1}{2}}\left(-e^{-i k R}+\tilde{S} e^{i k R}\right)
$$

As this point, it is important to state a convention which is used throughout this chapter. The wavefunction in the "bra" portion of bra-ket notation is not complexconjugated. This provides for a simplification of notation in more complicated formulae. (A more detailed explanation of this general aspect of the Kohn principle is provided by Mott and Massey [2, p. 116].) Also, elastic scattering is assumed throughout the remainder of this chapter when the $\mathbf{S}$ matrix is not printed in boldface type. 
The trial function $\tilde{\psi}$ is expanded in a linear combination of $L^{2}$ basis functions as well as two non- $L^{2}$ functions employed to enforce the inhomogeneous boundary condition as $R \rightarrow+\infty$ :

$$
\tilde{\psi}(R)=-u_{0}(R)+\sum_{l=1}^{N} c_{l} u_{l}(R) .
$$

The function $u_{0}$ is regular at the origin $(R=0)$ and has the proper asymptotic form for the particular scattering energy:

$$
\lim _{R \rightarrow+\infty} u_{0}(R)=k^{-\frac{1}{2}} e^{-i k R}
$$

The regularization at the origin is usually accomplished through the use of a multiplicative switching function $f$ which has the asymptotic forms

$$
f(R)= \begin{cases}0 & \text { if } R=0 \\ 1 & \text { as } R \rightarrow+\infty\end{cases}
$$

The function $u_{1}(R)=u_{0}^{*}(R)$, which provides the out-going wave boundary condition. The remaining set of functions $\left\{u_{l}\right\}$, where $l=2,3,4, \ldots, N$, are simply the basis of $L^{2}$ functions chosen to span the interaction region.

The variational procedure determines the coefficients $\left\{c_{l}\right\}$. This is accomplished by substituting (2.4) into (2.2) and determining the extremum of $\tilde{S}$ by solving the system of simultaneous linear equations given by

$$
\frac{\partial}{\partial c_{l}} S[\tilde{\psi}]=0
$$

Having found the expansion coefficients $\left\{c_{l}\right\}$, these are then substituted back into (2.2) to give the final value for the $S$ matrix. When this procedure is done in its general form, the formula for $S$ becomes

$$
S=i\left(M_{00}-\mathbf{M}_{0}^{T} \cdot \mathbf{M}^{-1} \cdot \mathrm{M}_{0}\right)
$$

where

$$
\begin{aligned}
M_{00} & =\left\langle u_{0}|\hat{H}-\hat{E}| u_{0}\right\rangle \\
\left(\mathbf{M}_{0}\right)_{l} & =\left\langle u_{l}|\hat{H}-\hat{E}| u_{0}\right\rangle \\
\left(\mathbf{M}_{l l^{\prime}}\right. & =\left\langle u_{l}|\hat{H}-\hat{E}| u_{l^{\prime}}\right\rangle
\end{aligned}
$$


and $l, l^{\prime}=1, \ldots, N$.

Unfortunately, the matrix inversion $\mathrm{M}^{-1}$ in (2.8) is dependent on the scattering energy. In order to provide a more practical calculation, the energy-dependent portion of the $\mathbf{M}$ matrix is partitioned out using the Löwdin-Feshbach partitioning identity

$$
\left(\mathbf{M}^{-1}\right)_{Q Q}=\mathbf{M}_{Q Q}^{-1}+\mathbf{M}_{Q Q}^{-1} \mathbf{M}_{Q P}\left(\mathbf{M}_{P P}-\mathbf{M}_{P Q} \mathbf{M}_{Q Q}^{-1} \mathbf{M}_{Q P}\right)^{-1} \mathbf{M}_{P Q} \mathbf{M}_{Q Q}^{-1}
$$

in which the "P" subscript represents the partition containing energy-dependent $u_{1}$ function. This results in a more practical expression for $S$ given by

$$
S=i\left(B-\frac{C^{2}}{B^{*}}\right)
$$

where

$$
\begin{aligned}
& B=M_{00}-\mathbf{M}_{0}^{T} \cdot \mathbf{M}^{-1} \cdot \mathbf{M}_{0} \\
& C=M_{10}-\mathbf{M}_{0}^{* T} \cdot \mathbf{M}^{-1} \cdot \mathbf{M}_{0}
\end{aligned}
$$

in which

$$
M_{10}=\left\langle u_{1}|\hat{H}-\hat{E}| u_{0}\right\rangle
$$

and the matrices $\mathbf{M}_{0}$ and $\mathbf{M}$ are restricted to index values $l=2, \ldots, N$.

As mentioned previously, the $\mathrm{K}$ matrix formulation of the Kohn variational principle is known to be plagued by anomalous non-physical singularities, whereas the $\mathrm{S}$ matrix formulation presents no such problem. The reason for this difference is based on the different ways that the two methods impose the proper boundary conditions on the scattering wavefunction. A short discussion of the $\mathrm{K}$ matrix formulation is useful.

The proper expression for the functional in (2.1) when applied with $\mathrm{K}$ matrix boundary conditions is give by

$$
K[\tilde{\psi}]=\tilde{K}-2\langle\tilde{\psi}|\mathbf{H}-\mathbf{E}| \tilde{\psi}\rangle
$$

Here, the trial wavefunction $\tilde{\psi}$ is also regular at $R=0$, but the asymptotic form is given as

$$
\lim _{R \rightarrow+\infty} \tilde{\psi}(R)=k^{-\frac{1}{2}}[\sin (k R)+\tilde{K} \cos (k R)]
$$


There is a corresponding difference in the non- $L^{2}$ portion of the basis set. An expansion of the wavefunction similar to that given in (2.4) is employed, but the functions $u_{0}$ and $u_{1}$ are replaced with functions $\tilde{u}_{0}$ and $\tilde{u}_{1}$, respectively. These functions are also regularized at the origin, but have the asymptotic forms

$$
\begin{aligned}
& \lim _{R \rightarrow+\infty} \tilde{u}_{0}(R)=k^{-\frac{1}{2}} \sin (k R) \\
& \lim _{R \rightarrow+\infty} \tilde{u}_{1}(R)=k^{-\frac{1}{2}} \cos (k R) .
\end{aligned}
$$

Using the same technique described above for calculating the $S$ matrix, the $\mathrm{K}$ matrix is found to be

$$
K=-2\left(M_{00}-\mathbf{M}_{0}^{T} \cdot \mathbf{M}^{-1} \cdot \mathbf{M}_{0}\right)
$$

where the new matrices $M_{00}, \mathbf{M}_{0}$, and $\mathbf{M}$ are now calculated using $\tilde{u}_{0}$ and $\tilde{u}_{1}$ instead of $u_{0}$ and $u_{1}$.

Singularities in such a computation will occur when the matrix $\mathbf{M}$ is singular and cannot be inverted. It is immediately apparent that the matrix $M$ found in the $\mathrm{K}$ matrix formulation is real symmetric, and hence all of its eigenvalues are real. These eigenvalues are those of the matrix $\mathbf{H}-\mathbf{E}$. And so singularities occur when the total scattering energy $E$ corresponds to one of the eigenvalues of $\mathbf{H}$.

On the other hand, in the $S$ matrix formulation the matrix $M$ of $(2.8)$ is complex-symmetric. Since the eigenvalues of the corresponding Hamiltonian $\mathbf{H}$ are not real, singularities as a function of scattering energy $E$ cannot occur regardless of the parameters used in this calculation. The values of $E$ which cause the $\mathrm{S}$ matrix formulation of $\mathbf{M}$ to be singular are the solutions to the equation

$$
|\mathbf{M}|=0
$$

These values of $E$ are the Siegert eigenvalues [60] which characterize scattering resonances. These are complex energy poles of the $S$ matrix which have a finite imaginary part $-i \Gamma / 2$ indicating the lifetime $1 / \Gamma$ of the resonance. Therefore, the $S$ matrix formulation is free of the anomalous singularities which plague the $\mathrm{K}$ matrix formulation. 


\subsection{Static Exchange $e^{-}+H$ Scattering}

In this section, the $\mathrm{S}$ matrix formulation of the Kohn variational principle is applied to S-wave elastic scattering of $e^{-}+H$ where the hydrogen atom is in a $\phi_{1 s}$ state and a static exchange approximation is used. Again, atomic units are assumed. The static exchange approximation assumes that the bound electron remains in a $\phi_{1 s}$ state without any distortion (i.e. a mean field approximation), while still assuming a properly symmetrized form for the total spatial wavefunction based upon the electron spin state of the system, $S=0$ (singlet), or $S=1$ (triplet). (A review of this approximation can be found in reviews by Mott and Massey [2, p. 522], and Burke and Smith [11, p. 471].)

The reduced dimensionality Schrödinger equation for the scattering electron is found to be

$$
\left[-\frac{1}{2} \frac{d^{2}}{d R^{2}}+V-E_{1}\right] \psi(R)=0
$$

where $E_{1}$ is the asymptotic translational energy of the scattering electron and therefore $E_{\text {total }}=E_{1}+\epsilon_{1 s}$ is the total energy of the system. $\left(\epsilon_{1 s}=-\frac{1}{2}\right.$ is the energy of a 1s hydrogen atom.) The potential $V=V_{0}+W$ is composed of a local portion $V_{0}$ given by

$$
V_{0}(R)=-\left(1+\frac{1}{R}\right) e^{-2 R}
$$

together with a non-local exchange portion $W$ which operates on a general function to give

$$
W f(R)=\int_{0}^{\infty} w\left(R, R^{\prime}\right) f\left(R^{\prime}\right) d R^{\prime}
$$

in which the kernal $w\left(R, R^{\prime}\right)$ is given by

$$
w\left(R, R^{\prime}\right)=-4(-1)^{S}\left(E_{1}-\epsilon_{1 s}-\frac{1}{R_{>}}\right) R e^{-R} R^{\prime} e^{-R^{\prime}} .
$$

Here, $S=0$ for singlet scattering and $S=1$ for triplet scattering.

The choice of $L^{2}$ basis set is identical to that of Apagyi, Lévay, and Ladányi [50]:

$$
u_{l}(R)=R^{l-1} e^{-\alpha R}
$$


where $\alpha$ is a non-linear tunable parameter and $l=2,3, \ldots, N$. The non- $L^{2}$ functions are chosen to be

$$
\begin{aligned}
& u_{0}(R)=k^{-\frac{1}{2}}\left(1-e^{-\alpha R}\right) e^{-\mathrm{i} k R} \\
& u_{1}(R)=u_{0}^{*}(R)
\end{aligned}
$$

where the simple function $f(R)=1-e^{-\alpha R}$ is chosen as a regularizing switching function.

\subsection{Results and Discussion}

The calculation of Apagyi, Lévay, and Ladányi [50] studied elastic scattering of $e^{-}+H$ using the Schwinger variational principle with a static exchange approximation. Figure 2.1 shows values of $\tan \delta$ for singlet scattering at $k=1$ au for various values of the non-linear scale parameter $\alpha$. Figure 2.1a shows the results of the Schwinger variational study of Apagyi et al. [50] ; Figure 2.1b shows the results of the present $S$ matrix Kohn method. As indicated in the caption, basis set sizes are identical in both cases. Anomalous singularities are readily apparent in Figure 2.1a, increasing in number as the size of the basis set is increased. By contrast, the results of the $\mathrm{S}$ matrix Kohn calculation are quite stable over a wide range of $\alpha$ values. Figure 2.2 shows results for the same Kohn calculation over a wider range of $\alpha$. The stability of the calculation occurs over a wider range of $\alpha$ as the size of the basis set increases.

Figure 2.3 once again compares singlet results from the Schwinger calculation of Apagyi (Figure 2.3a) to those of the present $S$ matrix Kohn calculation (Figure 2.3b), this time holding the value of the non-linear parameter $\alpha$ constant while varying the asymptotic kinetic energy. Once again, the Schwinger principle reveals the presence of non-physical singularities while the Kohn calculation does not.

The singularity located at $k=0.28$ au is a "natural" singularity resulting from the phase shift $\delta$ passing through a multiple of $\frac{\pi}{2}$. This demonstrates the 

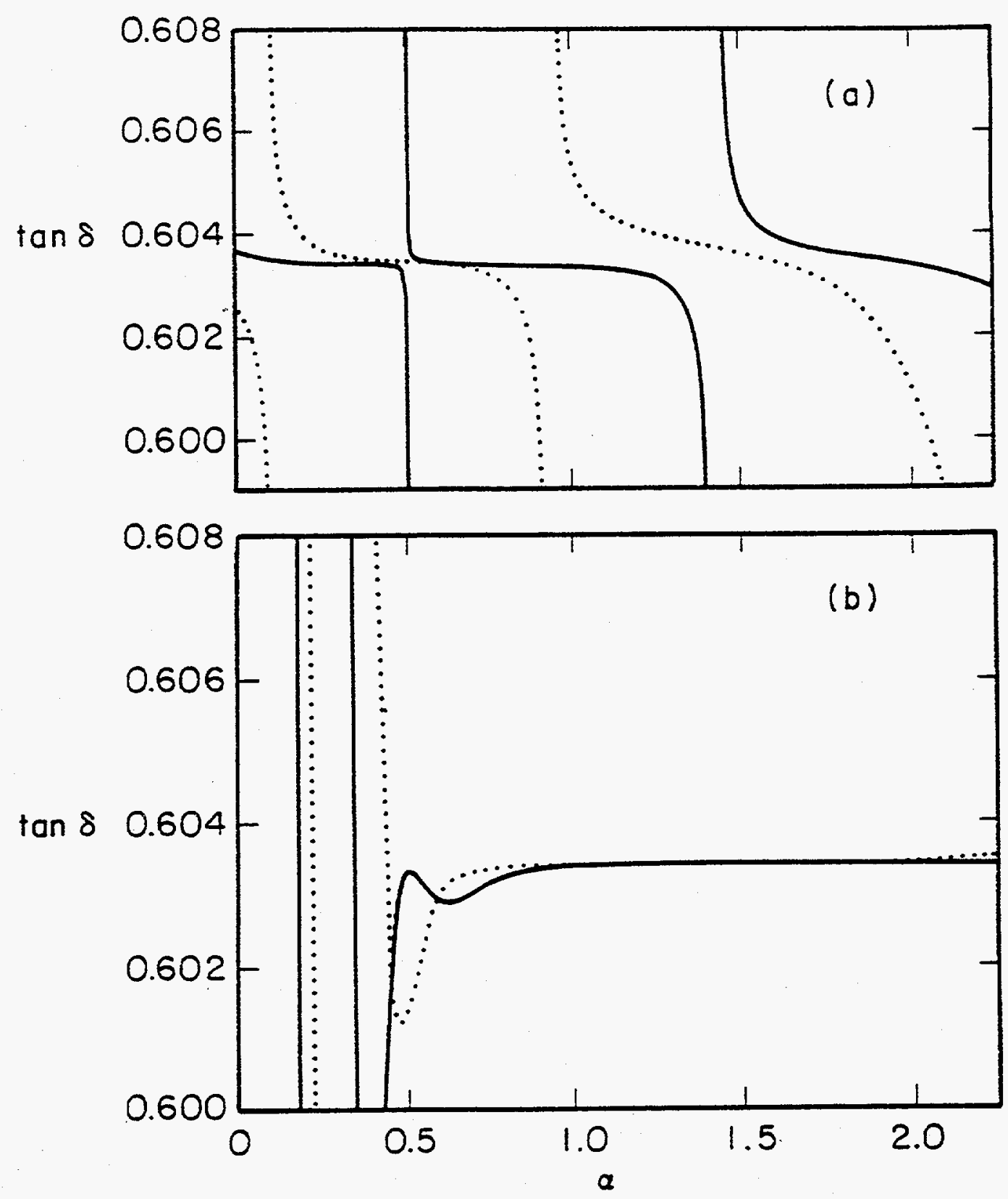

Figure 2.1: $\tan \delta$ as a function of $\alpha$ for $S=0$ scattering at $k=1$ au. (a) Schwinger principle; (b) S matrix Kohn principle. Dotted line: $N=5 L^{2}$ functions; Solid line: $N=6 L^{2}$ functions. Curves in (a) for $\alpha<0.5$ are continuous (see Figure 2.2) and have no singularities. 


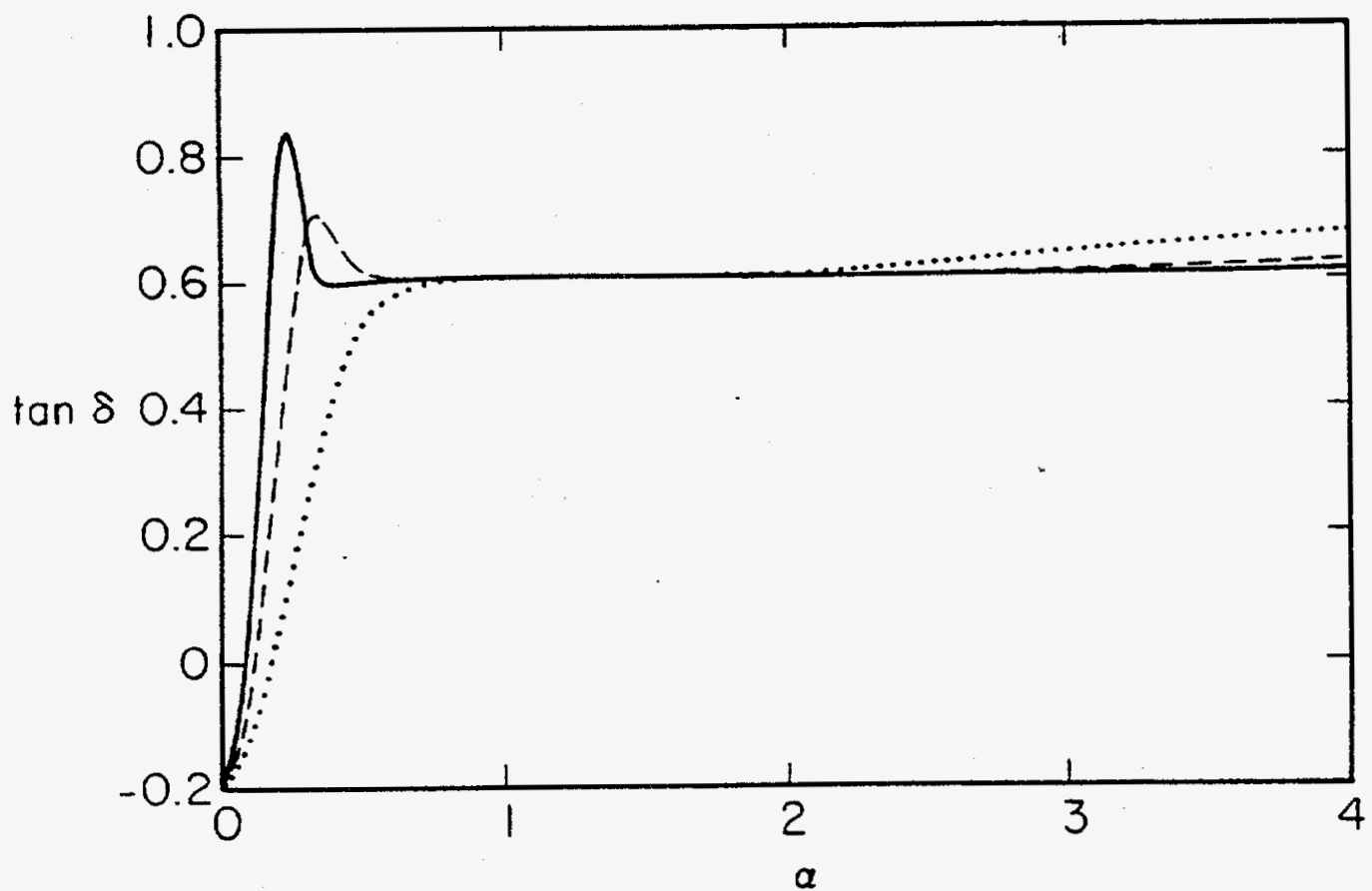

Figure 2.2: Same quantity as in Figure 2.1 (for $\mathrm{S}$ matrix Kohn method only) over a wider range of $\alpha$. Dotted line: $N=2$; Dashed line: $N=4$; Solid line: $N=6$.

difficulty of performing calculations in which anomalous singularities can appear haphazardly, particularly where a fully-converged basis set of great size cannot be employed. It becomes difficult to differentiate between fictitious singularities and actual resonance behavior.

Figure 2.4 demonstrates the stability of the $\mathrm{S}$ matrix Kohn calculation over a wide range of energy with a moderately-sized basis set and a fixed value of the nonlinear parameter $\alpha$. As one can see, convergence is achieved rather quickly. Finally, Figure 2.5 shows results from the application of the $S$ matrix Kohn method to triplet scattering at a constant value of $\alpha$ for many different basis set sizes. Once again, there is a singularity at $k=0.83$ an which is not anomalous. (The results of Apagyi et al. are not shown because they are indistinguishable from the present results when $N=8$.)

The discovery of anomalous singularities in the Schwinger variational principle is an interesting development. Studies by Winstead and McKoy [60], and Ladányi, Lévay, and Apagyi [61] have shown that these non-physical singularities occur when 

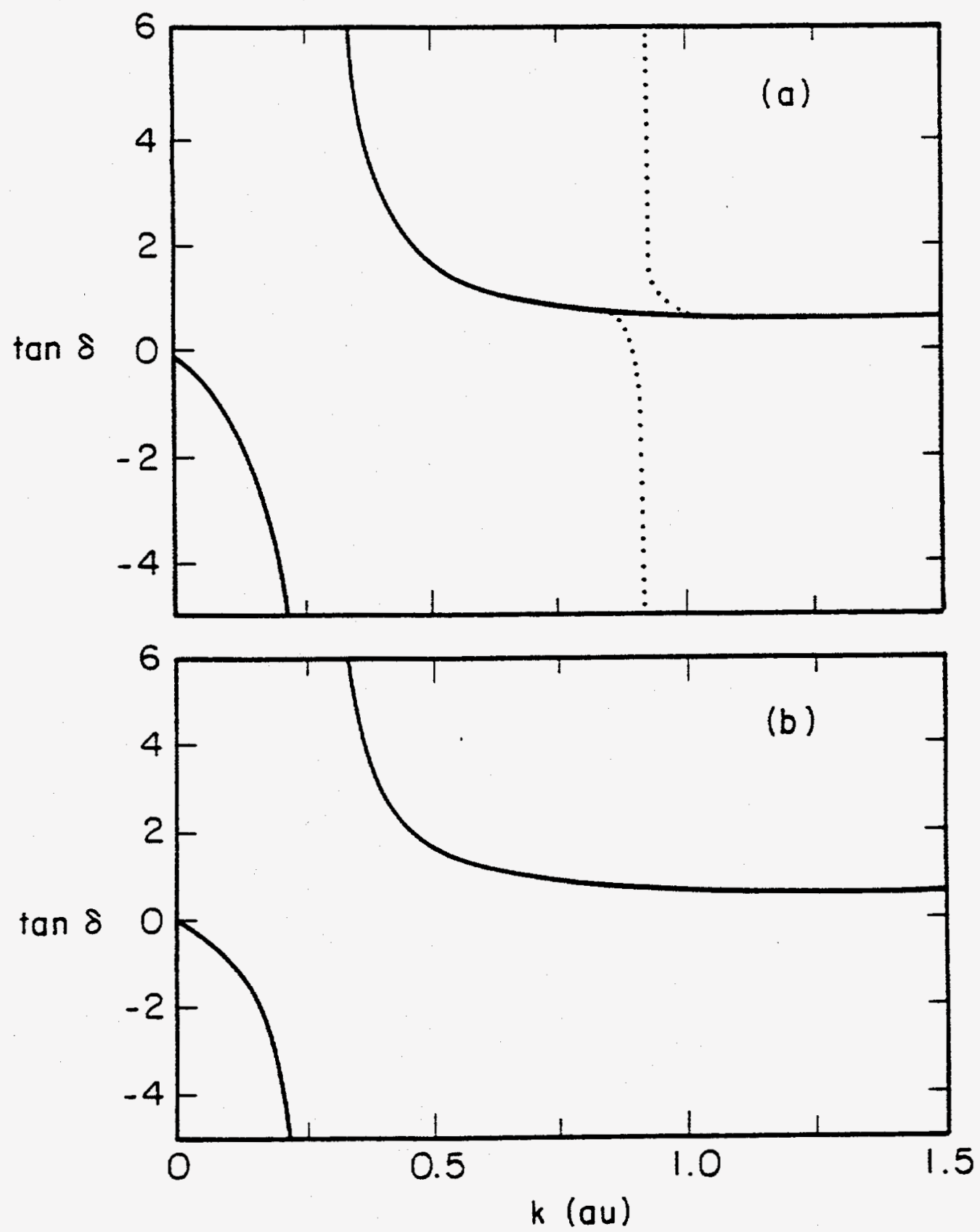

Figure 2.3: $\tan \delta$ as a function of $k$ for $S=0$ scattering with $\alpha=0.94$. (a) Schwinger principle; (b) S matrix Kohn principle. Dotted line: $N=5$; Solid line: $N=6$. (Dotted and solid lines are coincident in Figure 2.3b.) 


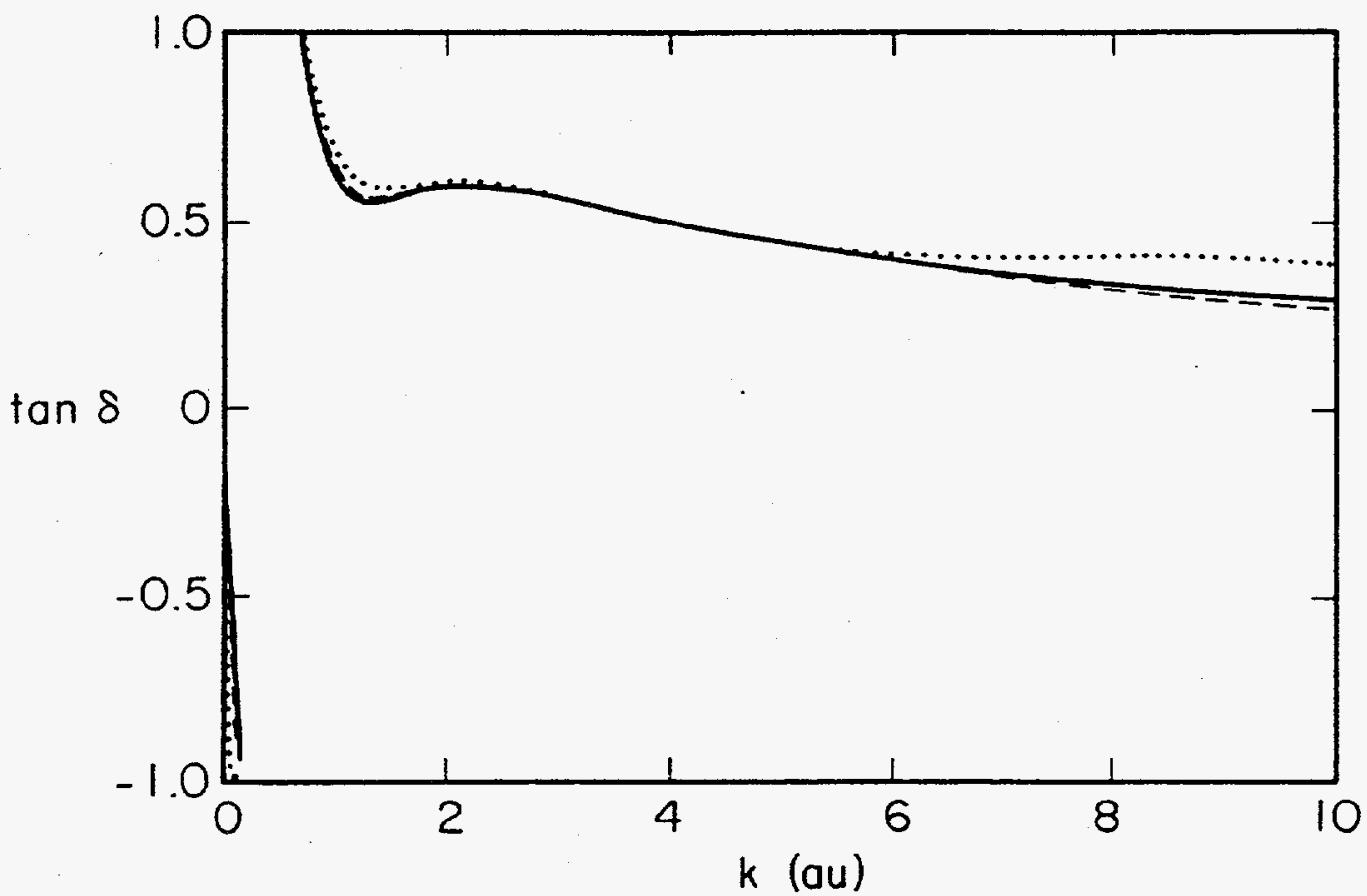

Figure 2.4: Same quantity as in Figure 2.3 for $S$ matrix Kohn method with $\alpha=4.0$ over a wider range of $k$. Dotted line: $N=2$; Dashed line: $N=4$; Dot-dash line: $N=6$; Solid line: $N=10$. The resonance singularity in $\tan \delta$ seen at $k=0.28$ au is the same one seen in Figure 2.3. 


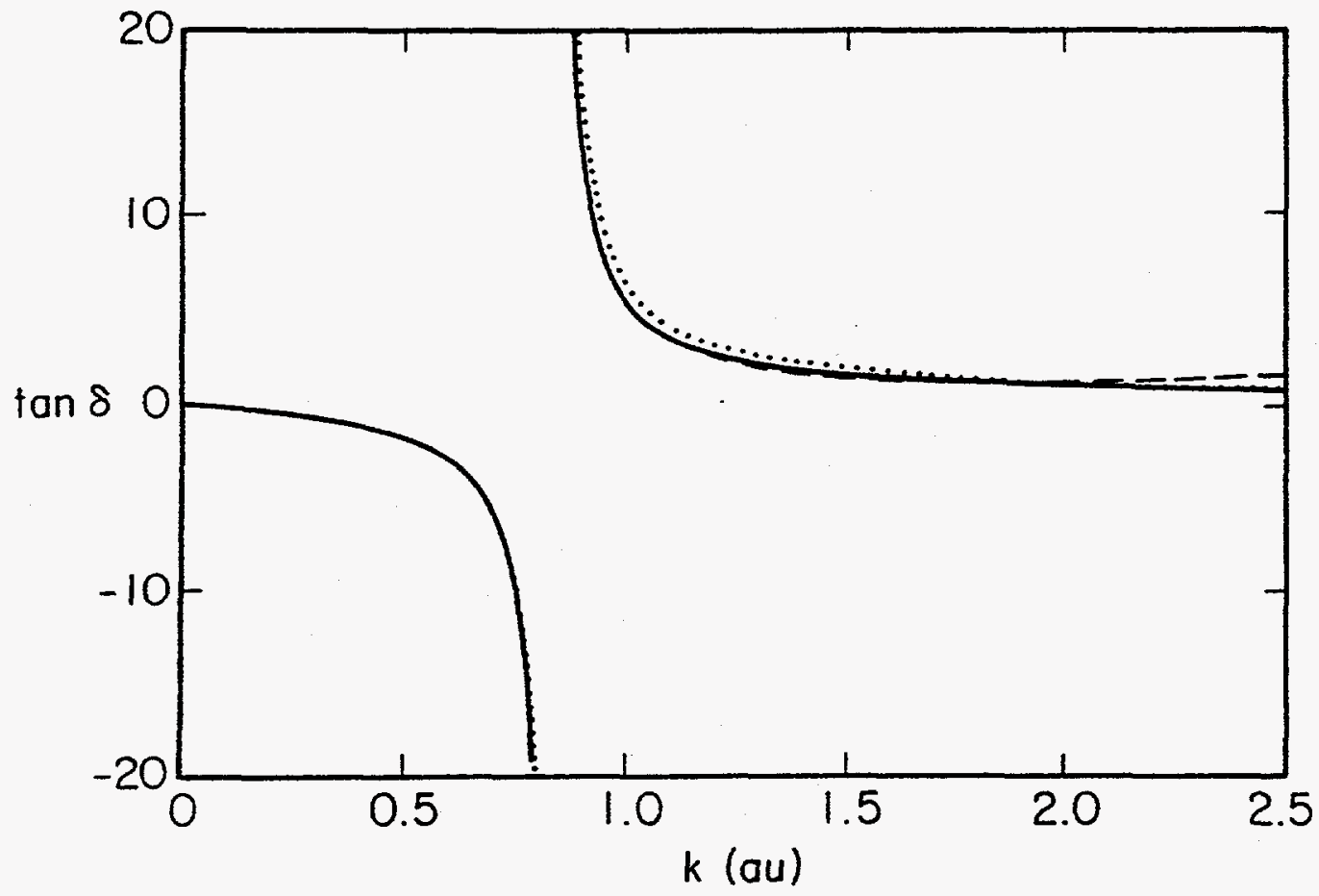

Figure 2.5: $\tan \delta$ as function of $k$ for triplet $(S=1)$ scattering with $\alpha=0.5$ for the $\mathrm{S}$ matrix Kohn method.

one of the eigenvalues of the potential matrix $\mathrm{V}$ vanishes. While one may construct any number of numerical procedures to avoid such a situation, the $\mathrm{S}$ matrix formulation of the Kohn variational principle does not require such effort. It therefore provides a more reliable numerical method for the study of quantum reactive scattering. 


\section{Chapter 3}

\section{The Theory}

\subsection{Introduction}

There exist a wide variety of methods by which discretized grids can be used to study the quantum mechanical wavefunctions of electrons in atoms. Baye and Heenen [63] discuss applications of generalized mesh methods for quantum mechanical problems, focusing first on grids associated with orthogonal polynomials and then generalizing to other grids. The technique involves the generation of interpolating Lagrange functions [42] derived from a set of chosen grid points. The discussion is confined to applications to bound state problems. For systems involving electrons bound in atoms, however, the presence of Coulomb or centrifugal forces at finite distances greatly weakens the accuracy of techniques based on any Gaussian quadrature rules. Vincke, Malegat, and Baye [64] present a technique which provides for regularization of these singularities to ensure an accurate quadrature approximation.

Unfortunately, the distribution of grid points which may be ideal for representing the wavefunction of an electron bound in an atom is probably not ideal for representing an electron scattering from an atom. Bound wavefunctions decay exponentially at moderate distances and do not require as high a grid density asymptotically as do free electrons with finite kinetic energy. But by the same token, there must be a sufficient density of grid points close to the origin in order to accurately approximate the usually crucial contribuution of S-wave scattering. Reconciling these 
two length scales presents a challenge. Unfortunately, grids which provide proper point densities for molecular scattering [41] do not provide adequate density near the Coulomb singularity region. And grids derived from orthogonal polynomials which provide high density near the Coulomb singularity region (such as a DVR derived from Laguerre functions) do not provide adequate density in the asymptotic region.

Recently, Botero and Shertzer [65] have used the finite-element method (FEM) $[66,67]$ to study elastic scattering of an electron from a hydrogen atom using radial grids which satisfy the constraints of both of these length scales. Poet [68] and Wang and Callaway [69] have used a Numerov propagation method to study electron-hydrogen scattering up to the $n=3$ threshold. In this chapter, a radial DVR is developed which satisfies the constraints resulting from the two length scales in the electron-hydrogen scattering system. In the next chapter, the scattering of an electron from a hydrogen atom $\left(J_{\text {total }}=0\right)$ between the $n=2$ and $n=3$ thresholds is studied with this DVR. The $\mathbf{S}$ matrix for the scattering system is calculated using the $\mathrm{S}$ matrix formulation of the Kohn variational principle derived by Zhang, Chu, and Miller [24].

\subsection{Hamiltonian and Coordinate System}

The objective is to study the scattering of a low energy electron from a hydrogen atom, restricted to a total energy $E$ between the $n=2$ and $n=3$ principal quantum states of the atom. The study is also restricted to a total angular momentum of $J=0$. As before, atomic units will be assumed throughout the discussion. Because the mass of the hydrogen atom nucleus is so much larger than those of the two electrons, it is assumed that the nucleus is infinitely large in this treatment. The choice of Jacobi coordinates is then equivalent to choosing the two radial coordinates $R_{1}$ and $R_{2}$ as the distances from each of the electrons to the nucleus. The single angle in the system $\gamma$, which results from eliminating the three extraneous Euler angles after restricting the total angular momentum to $J=0$, is the angle made by the two electrons with the nucleus as the center of rotation. 
The Hamiltonian for this system is

$$
\hat{H}=-\frac{1}{2} \frac{\partial^{2}}{\partial R_{1}^{2}}-\frac{1}{2} \frac{\partial^{2}}{\partial R_{2}^{2}}+\frac{\hat{L}^{2}}{2 R_{1}^{2}}+\frac{|\hat{J}-\hat{L}|^{2}}{2 R_{2}^{2}}-\frac{1}{R_{1}}-\frac{1}{R_{2}}+\frac{1}{\left|\mathbf{R}_{1}-\mathbf{R}_{2}\right|} .
$$

where $R_{1}$ and $R_{2}$ are arbitrarily taken to be the radial coordinates of the bound and scattering electrons (respectively). Here, $\hat{J}$ is the total angular momentum operator associated with both electrons and $\hat{L}$ is the angular momentum operator associated with the scattering electron. Setting $J$ equal to zero gives the form

$$
\hat{H}=-\frac{1}{2} \frac{\partial^{2}}{\partial R_{1}^{2}}-\frac{1}{2} \frac{\partial^{2}}{\partial R_{2}^{2}}+\left(\frac{1}{2 R_{1}^{2}}+\frac{1}{2 R_{2}^{2}}\right) \hat{L}^{2}-\frac{1}{R_{1}}-\frac{1}{R_{2}}+\frac{1}{\left|\mathbf{R}_{1}-\mathbf{R}_{2}\right|} .
$$

Here, $\hat{L}^{2}$ is the square of the orbital angular momentum operator associated with both electrons given by

$$
\hat{L}^{2}=-\left(\frac{\partial^{2}}{\partial \gamma^{2}}+\frac{1}{\tan \gamma} \frac{\partial}{\partial \gamma}\right)
$$

where $\gamma$ is the angle described previously at the beginning of the chapter. It will be noticed that the $\phi$ (azimuthal angle) dependence in this angular momentum operator can be ignored since $J=0$.

As has already been mentioned, it would be most useful to create a discrete variable representation (DVR) conducive to studying such a physical system in order to take advantage of the resultant sparcity of the Hamiltonian matrix as well as the simplicity of calculating potential matrix elements.

\subsection{Review of S Matrix Kohn Variational Princi- ple}

The $\mathbf{S}$ matrices (and accompanying state-to-state transition probabilities) are calculated using the complex version of the Kohn variational principle developed by Zhang, Chu, and Miller[24]. The multi-channel formulae which determine the computational procedure are given here without proof, as they are a simple generalization of the formulae derived in the previous chapter. The $\mathbf{S}$ matrix is given by

$$
\mathbf{S}=i\left(\mathbf{B}-\mathbf{C}^{T} \cdot \mathbf{B}^{*-1} \cdot \mathbf{C}\right)
$$


where $\mathbf{S}, \mathbf{B}$, and $\mathbf{C}$ are complex matrices whose dimension is equal to the number of open channels and $T$ denotes the transpose of a matrix. The individual matrices $B$ and $\mathbf{C}$ are given by

$$
\begin{aligned}
& \mathbf{B}=\mathbf{M}_{00}-\mathbf{M}_{0}^{T} \cdot \mathbf{M}^{-1} \cdot \mathbf{M}_{0} \\
& \mathbf{C}=\mathbf{M}_{10}-\mathbf{M}_{0}^{* T} \cdot \mathbf{M}^{-1} \cdot \mathbf{M}_{0}
\end{aligned}
$$

where $\mathbf{M}_{00}$ and $\mathbf{M}_{10}$ are also complex matrices whose dimension is equal to the number of open channels, and which are given by

$$
\begin{aligned}
& \left(\mathbf{M}_{\mathbf{0 0}}\right)_{\mathbf{n} \mathbf{n}^{\prime}}=\left\langle\Psi_{\mathbf{0 n}}|\hat{H}-\hat{E}| \Psi_{0 \mathbf{n}^{\prime}}\right\rangle, \\
& \left(\mathbf{M}_{10}\right)_{\mathbf{n n}^{\prime}}=\left\langle\Psi_{\mathbf{1 n}}|\hat{H}-\hat{E}| \Psi_{0 \mathbf{n}^{\prime}}\right\rangle .
\end{aligned}
$$

As was said before, the convention is used in which the "bra" is not complex conjugated in the Dirac notation. The $\Psi_{0 n}$ wavefunction is a so-called "free" function which has the asymptotic form

$$
\Psi_{0 \mathbf{n}}\left(R_{1}, R_{2}, \gamma\right) \sim k_{\mathbf{n}}^{-1 / 2} e^{-i\left[k_{\mathbf{n}} R_{1}-\left(\pi l_{\mathbf{n}} / 2\right)\right]} \phi_{\mathbf{n}}\left(R_{2}, \gamma\right) \quad \text { as } R_{1} \rightarrow+\infty .
$$

$k_{\mathbf{n}}$ is the magnitude of the asymptotic wavevector for the $\mathbf{n} t h$ open channel given by $k_{\mathbf{n}}=\sqrt{2\left(E-\epsilon_{\mathbf{n}}\right)}$ where $\epsilon_{\mathbf{n}}$ is the energy of the $\mathbf{n} t h$ hydrogen bound state. $l_{\mathbf{n}}$ is the orbital angular momentum quantum number for the $\mathbf{n} t h$ open channel, and $\phi_{\mathbf{n}}$ is the wavefunction for the hydrogen bound state associated with this channel. $\Psi_{1 \mathrm{n}}$ is simply the complex conjugate of $\Psi_{0 n}$. There is also the requirement that both of these wavefunctions vanish at the origin. The $M$ matrix is the so-called "bound-bound" matrix:

$$
\mathbf{M}_{\mathbf{t} \mathbf{t}^{\prime}}=\left\langle u_{\mathbf{t}}|\hat{H}-\hat{E}| u_{\mathbf{t}^{\prime}}\right\rangle
$$

in which the $u_{t}$ functions are $L^{2}$ functions which span the full dimensionality of the interaction region. As such, the $t$ indices are composite in nature and actually represent three separate indices (one for each spatial degree of freedom present in the system), so that $\mathbf{t}=\left\{t_{1}, t_{2}, t_{3}\right\}$. The $\mathbf{M}_{0}$ matrix is the "bound-free" matrix which provides the connection between the $L^{2}$ functions and the free functions. It is a rectangular matrix with the form

$$
\left(\mathbf{M}_{0}\right)_{\mathbf{t n}}=\left\langle u_{\boldsymbol{t}}|\hat{H}-\hat{E}| \Psi_{0 \mathbf{n}}\right\rangle .
$$




\subsection{DVR for the Interaction Region}

\subsubsection{Necessary Characteristics}

This section discusses the characteristics of the interaction region's discrete variable representation which are necessary for an accurate treatment of the $\mathrm{H}+\mathrm{e}^{-}$ scattering system. The most important feature of the DVR which must be kept in mind for this particular system is that the only manifestation of both the potential energy surface and the form of the free functions present within the interaction region occurs at the DVR grid points and nowhere else. Herein lies the inherently approximate nature of DVR methods.

As was mentioned previously in the introductory chapter, this is particularly a problem in electron-atom scattering because of the presence of grid density requirements both near the origin and at the edge of the interaction region. A high density of grid points is needed near the origin in order that the DVR approximation of the electron-nuclear potential will accurately reflect the strongly singular behavior of an attractive Coulomb interaction. This is particularly crucial in the case of S-wave scattering since the lack of a centrifugal barrier in such a case allows very high electron densities near the origin. The singularity of the potential at the origin ensures that there will be a very high local kinetic energy near this region. The rapid oscillations of the wavefunction in this region demand that a high enough density of DVR points be present in order to provide an accurate representation.

A counterpart of this constraint is that the Coulomb repulsion between the electrons must also be accurately reflected by the choice of DVR points. Of course, the regions of the potential energy surface where there is very strong repulsion between the electrons will not figure too prominently in the calculation since the amplitude of the total wavefunction in these regions is very likely to be zero. Therefore, composite DVR points which are located in these regions can be safely omitted from the calculation.

A more important constraint on the choice of the DVR grid involves the requirement of a certain minimum density of points at the edge of the interaction region where the free electron is not located within the density cloud of the bound 
electron. The density of grid points required in this region is strongly dependent on the asymptotic kinetic energy of the free electron, since it is this which determines the frequency of oscillation in the wavefunction in this region. This is a constraint which in some ways is unique to scattering systems and their accompanying inhomogeneous boundary conditions. Although the size of the interaction region in the similar bound state system is comparable, the form of the wavefunction at the edge of the interaction region is invariably in the form of a decaying exponential, which is more effectively approximated by a section of DVR points which is locally sparse. Unfortunately this is generally not adequate in the case of a scattering system.

A more global consideration which needs to be taken into account in choosing an optimal DVR grid is the actual extent of the interaction region in the system, since it is throughout this entire region that the grid needs to adequately represent the wavefunction. Unfortunately, the presence of charged species in the $H+e^{-}$system introduces long-range forces into the potential energy surface which serve to increase the interaction region (and hence also increase the required size of the grid). The Coulomb interactions between the species make the potential energy surface considerably less well-behaved than that of a typical molecular scattering system in which the scattering atomic species are often uncharged and therefore have weaker interactions between each other (such as van der Waals interactions between molecules). But these considerations tend to dictate the choice of free functions used rather than the actual form of the DVR grid in the interaction region, since the general pattern of point densities present is generally not sensitive to the size of the interaction region. This point will be discussed later.

\subsubsection{Sturmian Functions and Potential Optimized DVR}

One of the factors which once plagued physicists trying to calculate energy levels of atoms (even ones with few electrons, such as helium) was the fact that basis sets composed of simple hydrogen atom eigenfunction expansions would offer only slowly converging results. Unfortunately, a basis set composed only of such functions is not complete without inclusion of continuum states $[18,70]$. 
The historical development of a set of functions which have proven to be an excellent basis set for attaining energy levels of simple atoms such as hydrogen is now examined. So-called "Sturmian" functions (a term coined by Rotenberg [71]) have been shown to offer an excellent alternative [72] to conventional hydrogen eigenfunction expansions [73] in the calculation of ground state helium eigenvalues. Their usefulness when combined with discrete variable representation methods in calculating eigenstates of molecular vibrational potentials [74] and of molecular van der Waals complexes [35] has also been demonstrated.

Sturmian functions associated with the Coulomb potential [75] solve the second-order differential equation

$$
\left(-\frac{1}{2} \frac{d^{2}}{d R^{2}}+\frac{l(l+1)}{2 R^{2}}-\frac{\alpha_{n l}}{R}-E\right) S_{n l}(R)=0
$$

in which the individual $S_{n l}(R)$ functions satisfy the standard boundary conditions

$$
\begin{aligned}
S_{n l}(0) & =0 \\
\lim _{R \rightarrow \infty} S_{n l}(R) & =0
\end{aligned}
$$

and $\alpha_{n l}=k n$ where $k=\sqrt{-2 E}$. The analytical form taken by the Sturmian function is

$$
S_{n l}=N_{n l} e^{-k R}(2 k R)^{l+1} L_{n-l-1}^{(2 l+1)}(2 k R)
$$

where

$$
N_{n l}=\left(\frac{k}{n} \frac{(n-l-1) !}{(n+l) !}\right)^{\frac{1}{2}}
$$

and $L_{n-l-1}^{(2 l+1)}$ is a Laguerre polynomial defined by [37, p. 775$]$

$$
L_{n-l-1}^{(2 l+1)}(x)=\sum_{m=0}^{n-l-1}(-1)^{m}\left(\begin{array}{c}
n+l \\
n-l-1-m
\end{array}\right) \frac{x^{m}}{m !} .
$$

Of course, there is an immediately apparent similarity found in the radial portion of the hydrogen atom eigenfunctions given by

$$
u_{n l}=\frac{1}{n} \sqrt{\frac{(n-l-1) !}{(n+l) !}} \rho^{l+1} e^{-\rho / 2} L_{n-l-1}^{(2 l+1)}(\rho)
$$


where $\rho=2 R / n$. The most apparent difference between (3.15) and (3.18) is the presence of a scaling factor in the argument of the Laguerre polynomial which is dependent upon the principal quantum number $n$. It is then seen that the effect of the parameter $\alpha_{n l}$ on the "eigenfunctions" of the "Coulomb" potential in (3.12) is to "pull" the corresponding $S_{n l}$ particle density much closer to the origin. This has the desirable effects of including continuum states in the Sturmian expansion which would not be present in a simple hydrogen atom eigenstate expansion, as well as confining the particle density of the basis set to a region more representative of the spatial extent of electrons bound within atoms.

Just as a variable "strength" parameter multiplying an ordinary Coulomb potential can be seen to "pull" its eigenfunctions closer to the origin, one may surmise that a similar multiplicative factor applied to a typical electron-atom scattering potential would have a similar effect. This is the subject of the next section.

\subsubsection{Radial DVR for Screened Coulomb Potentials}

Just as the usefulness of Sturmian functions as a basis set for calculating energy levels of atoms may be seen as resulting from their connection to a Coulomb potential, it should be possible to determine a potential more representative of an electron-atom scattering system and then determine eigenfunctions of that potential for use as a basis set in such scattering calculations. Since this work focuses on low energy electron scattering from a hydrogen atom in either the $1 \mathrm{~s}, 2 \mathrm{~s}$, or $2 \mathrm{p}$ states, it is worthwhile to confine the search for such a potential accordingly.

In the previous chapter a calculation was performed which examined elastic scattering of an electron from a hydrogen atom in its ground state. The static exchange approximation [76] was used in an effort to eliminate the radial degree of freedom associated with the bound electron, which was assumed to be fixed in the $\phi_{1 s}$ state. The local portion of the potential in this calculation

$$
V_{l o c a l}(R)=-e^{-2 R}\left(1+\frac{1}{R}\right)
$$

appears to offer an attractive alternative to the Coulomb potential for the purposes of correctly representing the most important physical characteristics of the scattering 
system. The scattering electron feels the full strength of the nucleus when it has penetrated to within infinitesimal distances from the nucleus. This is reflected in $V_{\text {local }}(R)$ since

$$
\lim _{R \rightarrow 0^{+}} V_{\text {local }}(R) \sim-\left(\frac{1}{R}\right) .
$$

This characteristic is certainly a necessary one for any potential which is chosen to determine the basis set. But we see that $V_{l o c a l}(R)$ also has very desirable characteristics in the asymptotic region as well, since

$$
\lim _{R \rightarrow \infty} V_{\text {local }}(R) \sim-e^{-2 R} .
$$

This Yukawa-like behavior reflects the shielding effect of the bound electron on the scattered electron. This characteristic ensures that eigenfunctions of this potential will exhibit free particle behavior in the near asymptotic region. Therefore, it seems that finding eigenvalues of $V_{\text {local }}(R)$ would provide an excellent basis for an electronatom scattering calculation.

But there are two main difficulties with this approach. First, the eigenstates of $V_{l o c a l}(R)$ are not $L^{2}$ functions above the ionization threshold. Secondly, only scattering from a $\phi_{1 s}$ state will be accurately represented by such a basis. An electron scattering from either the $\phi_{2 s}$ or $\phi_{2 p}$ states will feel an attractive potential at values of the scattering coordinate $R$ at which $V_{\text {local }}(R)$ is already in the Yukawa-like region. This is obviously because the shielding effect of the $\phi_{1 s}$ electron is sustained at distances where both the $\phi_{2 s}$ and $\phi_{2 p}$ electron clouds have been penetrated.

Solving the first problem is simple. It is sufficient to choose a basis set for the eigenvalue calculation which is composed solely of $L^{2}$ functions whose extent is no greater than a predetermined limit (presumably no greater than the interaction region for the scattering system). This has the effect of imposing a "hard wall" at the predetermined limit, causing successively higher eigenfunctions to emulate particle-ina-box eigenfunctions, and thereby attain greater oscillation in the interaction region than if low-index non- $L^{2}$ continuum functions were permitted.

The obvious solution to the second problem is to use the $V_{\text {local }}(R)$ associated with scattering from a static $\phi_{2 s}$ state for the eigenfunction calculation. For any given 
value of the scattering coordinate $R$, the potential energy $V_{\text {local }(2 s)}(R)$ will be lower than that of $V_{\text {local }(1 s)}(R)$ and therefore the local kinetic energy will be higher. This will only serve to increase the frequency range spanned by the $\phi_{2 s}$-derived basis relative to that spanned by the $\phi_{1 s}$-derived basis.

To derive the local potential associated with scattering from a static $\phi_{2 s}$ state, one begins with the proper radial wavefunction

$$
\phi_{2 s}(R)=\frac{1}{2 \sqrt{2}} e^{-R / 2}\left(2 R-R^{2}\right) .
$$

Exchange is ignored, and the form of the two electron wavefunction is then

$$
\Psi\left(R_{1}, R_{2}\right)=\phi_{2 s}\left(R_{1}\right) f\left(R_{2}\right)
$$

Using the familiar Hamiltonian

$$
\hat{H}=-\frac{1}{2} \frac{\partial^{2}}{\partial R_{1}^{2}}-\frac{1}{2} \frac{\partial^{2}}{\partial R_{2}^{2}}+\frac{\hat{L}^{2}}{2 R_{1}^{2}}+\frac{|\hat{J}-\hat{L}|^{2}}{2 R_{2}^{2}}-\frac{1}{R_{1}}-\frac{1}{R_{2}}+\frac{1}{\left|\mathbf{R}_{1}-\mathbf{R}_{2}\right|}
$$

one sets $J$ and $L$ equal to zero and integrates out the static $R_{1}$ degree of freedom (together with the only angle $\gamma$ ) to get

$$
\left[-\frac{1}{2} \frac{d^{2}}{d R_{2}^{2}}-\frac{1}{R_{2}}+\left\langle\phi_{2 s}\left(R_{1}, \gamma\right)\left|\frac{1}{\left|\mathbf{R}_{1}-\mathbf{R}_{2}\right|}\right| \phi_{2 s}\left(R_{1}, \gamma\right)\right\rangle-\left(E-\epsilon_{2 s}\right)\right] f\left(R_{2}\right)=0
$$

where $\epsilon_{2 s}$ is the energy of the $\phi_{2 s}$ state. A multipole expansion on the inter-electron potential gives

$$
\frac{1}{\left|\mathbf{R}_{1}-\mathbf{R}_{2}\right|}=\sum_{l=0}^{\infty} \frac{R_{<}^{l}}{R_{>}^{l+1}} P_{l}(\cos \gamma)
$$

The spherical symmetry of the $\phi_{2 s}$ state ensures that only the $l=0$ term of the expansion contributes, giving

$$
\left[-\frac{1}{2} \frac{d^{2}}{d R_{2}^{2}}-e^{-R_{2}}\left(\frac{1}{R_{2}}+\frac{3}{4}+\frac{1}{4} R_{2}+\frac{1}{8} R_{2}^{2}\right)-\left(E-\epsilon_{2 s}\right)\right] f\left(R_{2}\right)=0 .
$$

The effective potential for scattering from a hydrogen atom in a static $2 \mathrm{~s}$ state is then

$$
V_{2 s}(R)=-e^{-R_{2}}\left(\frac{1}{R_{2}}+\frac{3}{4}+\frac{1}{4} R_{2}+\frac{1}{8} R_{2}^{2}\right)
$$

The effective static potentials for scattering from $\phi_{1 s}$ and $\phi_{2 s}$ hydrogen states are shown in Figure 3.1 below. 


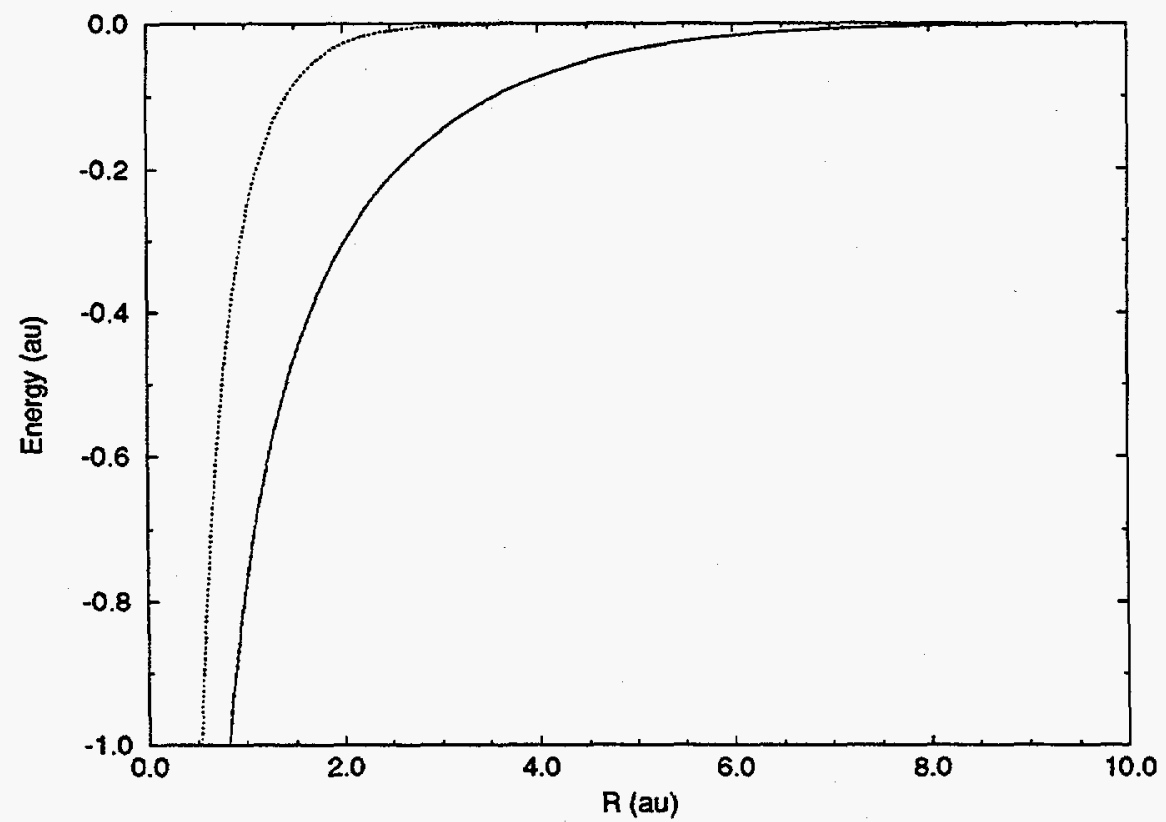

Figure 3.1: Effective potential for $e^{-}$scattering from a static $\phi_{1 s}$ or $\phi_{2 s} \mathrm{H}$ state. Dotted line: $V_{1 s} ;$ Solid line: $V_{2 s}$.

Just as the Coulomb potential (multiplied by a physically suitable scaling parameter $\alpha_{n l}$ ) can be diagonalized to provide Sturmian functions as an effective basis for bound state atom calculations, the previously derived effective static 2 s potential ought to provide an equally suitable basis set for electron-atom scattering calculations if the multiplicative scaling parameter $\alpha$ is chosen with care. Such a potential would then have the form

$$
\tilde{V}_{2 s}(R)=-\alpha e^{-R_{2}}\left(\frac{1}{R_{2}}+\frac{3}{4}+\frac{1}{4} R_{2}+\frac{1}{8} R_{2}^{2}\right) .
$$

This basis set can then be used to derive a potential-optimized DVR [77] ideal for electron-atom scattering. But a primary basis set must first be chosen with which the diagonalization of the static 2 s potential can be efficiently performed. The obvious choice for such a primary basis is Sturmian functions, mainly because of the great similarity between the Coulomb potential and the static 2 s potential near the origin.

The diagonalization begins with the designation of a set of orthogonalized 
(non-normalized) Sturmians of the form

$$
\phi_{n}(x)=e^{-x / 2} x L_{n}(x)
$$

where the $n$ index of the Laguerre polynomials is redefined from that in (3.17) and $\alpha=2$ such that

$$
L_{n}(x)=\sum_{k=0}^{n} \frac{(-1)^{k}}{k !}\left(\begin{array}{c}
n+2 \\
k+2
\end{array}\right) x^{k} .
$$

Recurrence relations governing these polynomials are given $[37, \mathrm{p} .782-3]$ as

$$
\begin{aligned}
(n+1) L_{n+1} & =(2 n+3-x) L_{n}-(n+2) L_{n-1} \\
x \frac{d}{d x} L_{n} & =n L_{n}-(n+2) L_{n-1} .
\end{aligned}
$$

A simple integration by parts gives the one-dimensional radial kinetic energy matrix as

$$
\hat{T}_{n m}=\frac{1}{2} \int_{0}^{\infty} d x \phi_{n}^{\prime}(x) \phi_{m}^{\prime}(x)
$$

where

$$
\phi_{n}^{\prime}(x)=-\frac{1}{2} e^{-x / 2} x L_{n}(x)+e^{-x / 2} L_{n}(x)+e^{-x / 2} x \frac{d}{d x} L_{n}(x) .
$$

Using the recurrence relations (3.33) this integral is given in terms of weighted moments of the Laguerre polynomials as

$$
\begin{aligned}
\int_{0}^{\infty} d x \phi_{n}^{\prime}(x) \phi_{m}^{\prime}(x)= \\
\quad \frac{1}{4}(n, 2, m)-\frac{1}{2}(n+1)(n, 1, m)+\frac{1}{2}(n+2)(n-1,1, m) \\
\quad-\frac{1}{2}(m+1)(n, 1, m)+(n+1)(m+1)(n, 0, m) \\
\quad-(n+2)(m+1)(n-1,0, m)+\frac{1}{2}(m+2)(n, 1, m-1) \\
\quad-(n+1)(m+2)(n, 0, m-1)+(n+2)(m+2)(n-1,0, m-1)
\end{aligned}
$$

where

$$
(n, t, m)=\int_{0}^{\infty} d x e^{-x} x^{t} L_{n}(x) L_{m}(x)
$$


The higher moments of the weighted Laguerre polynomials may be calculated by using (3.33) if the zeroth order moment $(n, 0, m)$ is known. Using (3.31) one obtains

$$
\begin{aligned}
(n, 0, m) & =\int_{0}^{\infty} d x e^{-x} L_{n}(x) L_{m}(x) \\
& =\sum_{k^{\prime}=0}^{m} \frac{(-1)^{k^{\prime}}}{k^{\prime} !}\left(\begin{array}{c}
m+2 \\
k^{\prime}+2
\end{array}\right) \sum_{k=0}^{n} \frac{(-1)^{k}}{k !}\left(\begin{array}{c}
n+2 \\
k+2
\end{array}\right)\left(k+k^{\prime}\right) ! \\
& =\sum_{k^{\prime}=0}^{m} C_{m k^{\prime}} \sum_{k=0}^{n} D_{n k k^{\prime}} .
\end{aligned}
$$

But

$$
\begin{aligned}
D_{n k k^{\prime}} & =\sum_{k=0}^{n} \frac{(-1)^{k}}{k !}\left(\begin{array}{c}
n+2 \\
k+2
\end{array}\right)\left(k+k^{\prime}\right) ! \\
& =\sum_{k=-2}^{n}(-1)^{k}\left(\begin{array}{c}
n+2 \\
k+2
\end{array}\right)\left[(k+1)(k+2) \cdots\left(k+k^{\prime}\right)\right] \\
& =\sum_{q=0}^{n+2}(-1)^{q}\left(\begin{array}{c}
n+2 \\
q
\end{array}\right)\left[(q-1)(q+0) \cdots\left(q+k^{\prime}-2\right)\right] \quad \text { if } k^{\prime} \geq 2 .
\end{aligned}
$$

But $\sum_{q=0}^{S}(-1)^{q}\left(\begin{array}{l}S \\ q\end{array}\right) q^{N}=0$ if $S-1 \geq N \geq 0$ with $N$ a non-negative integer $[78, \mathbf{p}$. 4]. Therefore, only the terms $D_{n k 0}$ and $D_{n k 1}$ are finite. It can easily be shown that

$$
\begin{aligned}
& \sum_{k=0}^{n} D_{n k 0}=n+1 \quad \text { and } \\
& \sum_{k=0}^{n} D_{n k 1}=1 .
\end{aligned}
$$

It is then found that for $n \geq m$

$$
\begin{aligned}
(n, 0, m) & =\sum_{k^{\prime}=0}^{1} C_{m k^{\prime}} \sum_{k=0}^{n} D_{n k k^{\prime}} \\
& =\frac{(-1)^{0}}{0 !}\left(\begin{array}{c}
m+2 \\
2
\end{array}\right)(n+1)+\frac{(-1)^{1}}{1 !}\left(\begin{array}{c}
m+2 \\
3
\end{array}\right) \cdot 1+0+0+\cdots \\
& =\frac{1}{6}(m+1)(m+2)(3 n-m+3)
\end{aligned}
$$


Using this zeroth order moment, the first order moment for the Laguerre polynomials is easily calculated for the case $(n \geq m)$ by

$$
\begin{aligned}
(n, 1, m) & =-(n+1)(n+1,0, m)+(2 n+3)(n, 0, m)-(n+2)(n-1,0, m) \\
& =\frac{1}{2}(m+1)(m+2) \quad(n \geq m) .
\end{aligned}
$$

A similar calculation gives the second order moment as

$$
(n, 2, m)=(m+1)(m+2) \delta_{n m} .
$$

Substituting into (3.33) and (3.36) gives

$$
\begin{aligned}
& \hat{T}_{n m}=\frac{1}{24}(m+1)(m+2)(4 m+3) \quad(n=m) \\
& \hat{T}_{n m}=\frac{1}{12}(m+1)(m+2)(2 m+3) \quad(n>m) .
\end{aligned}
$$

The second order moment gives the overlap integral for the non-normalized basis functions, and can be used to provide a normalization constant. Inclusion of a radial scale parameter $s$ and re-indexing the basis functions such that $n=1$ refers to the first function gives

$$
\phi_{n}(x)=\sqrt{\frac{s}{n(n+1)}} e^{-s x / 2} s x L_{n}(s x)
$$

where

$$
\begin{aligned}
n L_{n+1} & =(2 n+1-x) L_{n}-(n+1) L_{n-1} \quad \text { and } \\
x L_{n} & =-n L_{n+1}+(2 n+1) L_{n}-(n+1) L_{n-1}
\end{aligned}
$$

with $L_{1}=1, L_{2}=3-x$, etc. The recurrence relation for the entire basis function is

$$
\phi_{n+1}(x)=\frac{2 n+1-s x}{\sqrt{n(n+2)}} \phi_{n}(x)-\sqrt{\frac{(n-1)(n+1)}{n(n+2)}} \phi_{n-1}(x) .
$$

The final formulae for the one-dimensional radial kinetic energy in this Sturmian basis set is then given as

$$
T_{n m}^{S T U}= \begin{cases}\frac{s^{2}}{24}(4 n-1) & \text { if } n=m \\ \frac{s^{2}}{12}(2 m+1) \sqrt{\frac{m(m+1)}{n(n+1)}} & \text { if } n>m\end{cases}
$$


The overlap integral is simply

$$
O_{n m}^{S T U}=\delta_{n m} .
$$

The determination of a suitable radial scale parameter $s$ is dependent ultimately on how much spatial extent the primary basis will require in order to provide adequate coverage of the interaction region in the particular scattering system. This must ultimately be determined empirically through convergence tests within the final basis, but certainly the end of the interaction region provides a lower bound on the spatial extent of the primary basis. With this in mind, a cue is taken from the procedure used for calculating the abscissae in Gaussian quadrature formulae. Since the primary basis set being used in this diagonalization is composed of Laguerre polynomials multiplied by their proper weight functions, it seems reasonable to assume that choosing the scale factor such that the final Gaussian quadrature point $x_{n}$ (which would be used in integrating products of these basis functions) were placed just beyond the edge of the interaction region would ensure that the primary basis set put $L^{2}$ density in the proper location for the scattering system. The $n$ Gaussian quadrature points $[79$, p. 970] used for exactly integrating products of the primary basis functions are simply the zeroes of the Laguerre polynomial of degree $(n+1)$. Requiring that the final $n^{\text {th }}$ zero be placed at a particular value of the scattering coordinate determines the value of the scale parameter.

Since the radial kinetic energy has been determined analytically for the Sturmian basis, one must now calculate the potential matrix elements for the strengthened static 2 s potential. Recall that the analytic form of this potential is given by (3.29) as

$$
\tilde{V}_{2 s}(x)=-\alpha e^{-x}\left(\frac{1}{x}+\frac{3}{4}+\frac{1}{4} x+\frac{1}{8} x^{2}\right) .
$$

Since the lowest power of $x$ multiplying the decaying exponential in each of the functions of the primitive basis is $x^{1}$, it is easily seen that in spite of the presence of a $x^{-1}$ term in the potential, the potential matrix elements can be calculated exactly by Gauss-Laguerre quadrature. 


$$
\begin{aligned}
\tilde{V}_{n m}= & \int_{0}^{\infty} d x\left[\sqrt{\frac{s}{n(n+1)}} e^{-s x / 2} s x L_{n}(s x)\right] \cdot\left[-\alpha e^{-x}\left(\frac{1}{x}+\frac{3}{4}+\frac{1}{4} x+\frac{1}{8} x^{2}\right)\right] . \\
& {\left[\sqrt{\frac{s}{m(m+1)}} e^{-s x / 2} s x L_{m}(s x)\right] } \\
\propto & \int_{0}^{\infty} d x e^{-(s+1) x} P_{q}(x)
\end{aligned}
$$

where $P_{q}(x)$ is simply a polynomial in $x$ of degree $q \leq 2 \max (n, m)+2$. This technique forms the essential background for the regularization of unshielded Coulomb potentials in currently popular Laguerre mesh DVRs for atom eigenvalue calculations [64] .

As was mentioned previously, just as the Coulomb potential (multiplied by a strength parameter $\alpha_{n l}$ ) can be used to derive Sturmian functions as a basis set for atom eigenfunction calculations, the static 2 s potential can be used in the same manner to derive a secondary basis set suitable for electron-atom scattering calculations. But there is a difference which needs to be addressed. The strength parameter $\alpha_{n l}$ used in the derivation of the Sturmian functions is dependent on the "quantum" numbers of the resulting eigenfunctions. It would not be efficient to diagonalize the static $2 \mathrm{~s}$ potential for a whole host of strength parameters, especially since there may be a need for many eigenfunctions forming the secondary basis set for a given scattering problem. It would be preferred if a single strength parameter $\alpha$ could be chosen which offers a satisfactory compromise. Eigenfunctions would then be found for the potential energy function given by (3.29)

$$
\tilde{V}_{2 s}(R)=-\alpha e^{-R}\left(\frac{1}{R}+\frac{3}{4}+\frac{1}{4} R+\frac{1}{8} R^{2}\right) .
$$

The criteria for choosing such a parameter are now discussed. Obviously, choosing $\alpha=0$ would result in a series of eigenfunctions closely resembling particlein-a-box eigenfunctions (sine functions). This would be satisfactory if the system being studied did not have regions of the scattering coordinate in which the local kinetic energy of either the bound or scattering electron could become very large, since the Fourier transform of the resulting eigenfunctions would vanish above a certain wavelength. This would not be a serious constraint for scattering calculations 
involving potential energy surfaces which behave in a fairly benevolent fashion, such as many surfaces associated with molecular scattering.

But for electron-atom systems this is certainly not the case. In order to accurately represent the local kinetic energies present near the origin (when nuclear shielding is all but non-existent), the number of $\alpha=0$ eigenfunctions required would be quite large since only the highest such functions would have rapid enough oscillations near the origin to accurately represent the high kinetic energy there. In the same way that increasing the value of the $\alpha_{n l}$ parameter in the calculation of Sturmian functions "pulls" the eigenfunctions closer to the origin, thus enabling the basis set to span the needed continuum $[18,10]$, a "strengthened" static $2 \mathrm{~s}$ potential is needed to provide inclusion of the continuum for scattering systems.

On the other hand, if $\alpha$ is too large the eigenfunctions resulting from diagonalizing the strengthened static $2 s$ potential will have too much particle density near the origin and will not accurately span the region of the scattering coordinate at the edge of the interaction region. $\alpha$ could in fact be so strong that very few of the eigenfunctions will have eigenvalues above $V=0$. This would mean that the region of the scattering coordinate at the edge of the interaction region would be represented mainly by functions whose functional form was mainly decaying exponential in nature.

Obviously a scattering wavefunction with an inhomogeneous boundary condition will not be well-represented by such a basis set. In this sense one can think of $\alpha$ as a "tuning" parameter which is designed to reconcile the competing interests and requirements of the strong Coulomb behavior near the unshielded region of the scattering coordinate with the shielded Yukawa-like behavior of the potential felt by the scattering electron at the edge of the interaction region. Ultimately the choice of $\alpha$ is determined by which value gives the most converged results with the fewest eigenfunctions making up the secondary basis set. More will be discussed about the most efficient values for the $\alpha$ strength parameter later.

Assuming that an optimal $\alpha$ strength parameter has been found, the static 2s radial potential is diagonalized within the previously described Sturmian basis set. The orthogonal transformation matrix $Z_{1}$ which effects this diagonalization is then 
stored, as it provides the formulae for the eigenfunctions in terms of the Sturmian basis.

$$
\chi_{n}(x)=\sum_{t=1}^{N}\left(Z_{1}^{T}\right)_{n t} \phi_{t}(x)
$$

where $N$ is the total number of Sturmians used in the diagonalization and $T$ denotes transposition. (In actuality, only as many rows $n$ of $Z_{1}^{T}$ are stored as there are numbers of radial DVR points anticipated to be needed in the final calculation.)

The transformation of the secondary static 2 s eigenfunction basis into the tertiary radial DVR basis is now discussed. As has been discussed previously, the development of a diagonal representation of the potential energy matrix [25] has been well-characterized $[26,29]$. The more specific method of diagonalizing the position operator $\hat{x}$ within a particular basis to provide a DVR basis has been shown to provide excellent results [80], and it is used here also.

It is necessary to calculate the $\hat{x}$ matrix within the secondary basis set of static 2 s eigenfunctions. First, the $\hat{x}$ matrix is calculated within the primary basis set of Sturmian functions and then transformed into the secondary representation with the $Z_{1}$ matrix. In order to calculate the $\hat{x}$ matrix within the primary Sturmian basis, the third position moment of the previously-derived Laguerre functions is calculated. Using the recurrence expression derived for the Sturmian functions (3.47) and the fact that the second moment of the Laguerre functions is simply the overlap integral (3.49), it is a simple matter to derive that

$$
x_{n m}^{(1)}= \begin{cases}-\frac{1}{s} \sqrt{n^{2}-1} & \text { if } m=n-1 \\ \frac{1}{s}(2 n+1) & \text { if } m=n \\ -\frac{1}{s} \sqrt{n(n+2)} & \text { if } m=n+1 \\ 0 & \text { if }|n-m|>1\end{cases}
$$

where the superscript 1 indicates the representation of $\hat{x}$ in the primary Sturmian basis. Transformation of $\hat{x}$ into the static 2 s eigenfunction representation is simply accomplished with the $Z_{1}$ transformation matrix by

$$
\hat{\mathbf{x}}^{(2)}=\mathrm{Z}_{1}^{T} \cdot \hat{\mathbf{x}}^{(1)} \cdot \mathrm{Z}_{1}
$$

The position operator $\hat{\mathbf{x}}^{(2)}$ is then diagonalized within the secondary static 2 s eigenfunction basis to obtain a second transformation matrix $Z_{2}$. The product of these 
two transformation matrices

$$
\mathrm{Z}_{12}=\mathrm{Z}_{1} \cdot \mathrm{Z}_{2}
$$

is the only matrix which needs to be finally stored, and gives the transformation from the primary Sturmian basis to the final one-dimensional radial DVR basis. The explicit formula for a radial DVR basis function in terms of $x$ is then

$$
\varphi_{n}(x)=\sum_{t=1}^{N}\left(Z_{12}^{T}\right)_{n t} \phi_{t}(x) .
$$

Calculation of the one-dimensional radial kinetic energy in this new basis is then simply accomplished with

$$
\mathbf{T}^{D V R}=\mathbf{Z}_{12}^{T} \cdot \mathbf{T}^{S T U} \cdot \mathbf{Z}_{12}
$$

where $T^{S T U}$ is calculated with (3.48).

\subsubsection{Gauss-Legendre DVR for Angular Coordinate}

Although the single angular degree of freedom present in the system also contains singularities in the inter-electron potential at $\gamma=0$, no attempt was made to develop a specialized DVR for this degree of freedom, mainly because the cusp behavior at $\gamma=0$ is a repulsive cusp and it was anticipated that the quadrature approximation afforded by simple Gauss-Legendre quadrature would be adequate. The finite basis representation (FBR) functions chosen for the angular degree of freedom were spherical harmonics in which the azimuthal angle is integrated away:

$$
\psi_{n 0}(\gamma)=\sqrt{\frac{2 n-1}{2}} P_{n-1}(\cos \gamma)
$$

where $P_{n}$ is a Legendre polynomial $[37, \mathrm{p} .775]$ generated by the standard recurrence relation

$$
n P_{n}(x)=(2 n-1) x P_{n-1}(x)-(n-1) P_{n-2}(x)
$$

where $P_{0}(x)=1, P_{1}(x)=x$, etc. Notice that a re-indexing similar to that performed on the Laguerre polynomials has been done here too. Within this indexing convention, 
the squared angular momentum operator in the finite basis representation takes the form

$$
\begin{aligned}
\left(\mathbf{T}_{c}^{F B R}\right)_{n n^{\prime}} & =\left(\hat{L}^{2}\right)_{n n^{\prime}} \\
& =n(n-1) \delta_{n n^{\prime}}
\end{aligned}
$$

where the subscript " $c$ " indicates "centrifugal". The orthogonal matrix which transforms the angular functions into the localized Gauss-Legendre DVR representation is

$$
\begin{aligned}
A_{n i} & =\sqrt{\omega_{i}} \psi_{n 0}(\gamma) \\
& =\sqrt{\omega_{i}} \sqrt{\frac{2 n-1}{2}} P_{n-1}(\cos \gamma)
\end{aligned}
$$

where $\omega_{i}$ is the Gaussian quadrature weight associated with the $i^{\text {th }}$ DVR point. The localized angular DVR functions are then given as

$$
\xi_{n}(\gamma)=\sum_{t=1}^{N}\left(A^{T}\right)_{n t} \psi_{t 0}(\gamma)
$$

and the centrifugal energy term in the localized DVR representation is

$$
\mathbf{T}_{c}^{D V R}=\mathbf{A}^{T} \cdot \mathbf{T}_{c}^{F B R} \cdot \mathbf{A}
$$

where $T_{c}^{F B R}$ is given by (3.58).

\subsection{Form of the Symmetrized Hamiltonian}

\subsubsection{Benefits of Symmetrization}

The exploitation of symmetry $[81,82]$ within the representation of the Hamiltonian of the scattering system provides great benefits. Starting with the form of the Hamiltonian already given in (3.2), it is seen that it is symmetric with respect to exchange of the coordinates of each electron. In other words,

$$
\hat{\mathcal{X}}_{12} \hat{H}=\hat{H}
$$


The total wavefunction for the two electron system can be expanded in eigenstates of the total spin operator $\hat{S}$ to give

$$
\begin{aligned}
& \Psi_{T}\left(R_{1}, R_{2}, \gamma ; s_{1}, s_{2}\right)= \\
& \quad \sum_{s_{1}, s_{2}} \Psi_{+}\left(R_{1}, R_{2}, \gamma ; s_{1}, s_{2}\right) \sigma_{+}\left(s_{1}, s_{2}\right)+\Psi_{-}\left(R_{1}, R_{2}, \gamma ; s_{1}, s_{2}\right) \sigma_{-}\left(s_{1}, s_{2}\right)
\end{aligned}
$$

where the summation has been split into singlet and triplet groups, respectively,

$$
\begin{aligned}
& \sigma_{+}=\frac{1}{\sqrt{2}}\left[\alpha_{1} \beta_{2}-\beta_{1} \alpha_{2}\right] \\
& \sigma_{-}=\left\{\begin{array}{l}
\alpha_{1} \alpha_{2} \\
\frac{1}{\sqrt{2}}\left[\alpha_{1} \beta_{2}+\beta_{1} \alpha_{2}\right] \\
\beta_{1} \beta_{2} .
\end{array}\right.
\end{aligned}
$$

Electrons satisfy Fermi-Dirac statistics. Since the singlet spin state $\sigma_{+}$is antisymmetric with respect to exchange, the spatial function associated with it must be symmetric. Likewise, the three triplet spin states $\sigma_{-}$must be paired with antisymmetric spatial functions. Therefore

$$
\begin{aligned}
& \hat{\mathcal{X}}_{12} \Psi_{+}=\Psi_{+} \text {and } \\
& \hat{\mathcal{X}}_{12} \Psi_{-}=-\Psi_{-} .
\end{aligned}
$$

Using (3.63) and the fact that $\left[\hat{\mathcal{X}}_{12}, \hat{H}\right]=0$ it is seen that

$$
\begin{aligned}
\left\langle\Psi_{+}|\hat{H}| \Psi_{-}\right\rangle & =\left\langle\Psi_{+}\left|\hat{\mathcal{X}}_{12} \hat{H}\right| \Psi_{-}\right\rangle \\
& =\left\langle\Psi_{+}\left|\hat{H} \hat{\mathcal{X}}_{12}\right| \Psi_{-}\right\rangle \\
& =-\left\langle\Psi_{+}|\hat{H}| \Psi_{-}\right\rangle \\
& =0 .
\end{aligned}
$$

Therefore, isolating singlet and triplet portions of the spatial basis set block diagonalizes the Hamiltonian. Since the most computationally intensive part of the calculation of the $\mathbf{S}$ matrix is the inversion of the bound-bound Hamiltonian matrix in (3.5) and (3.6), the reduction in dimensionality of the Hamiltonian into two smaller matrices offers a considerable savings. 


\subsubsection{Calculation of the Hamiltonian Matrix Elements}

Since the Hamiltonian being used ignores spin-orbit coupling and has no dependence on the spins of the two electrons it is sufficient to restrict our basis set to functions of a purely spatial character. The most general expansion of the total wavefunction within the restricted $L^{2}$ space is then

$$
\Psi\left(R_{1}, R_{2}, \gamma\right)=\sum_{i j k} c_{i j k} \varphi_{i}\left(R_{1}\right) \varphi_{j}\left(R_{2}\right) \xi_{k}(\gamma)
$$

where $\varphi_{i}$ is defined by (3.54) and $\xi_{k}$ is defined by (3.61). Then

$$
\begin{aligned}
\hat{\mathcal{X}}_{12} \Psi\left(R_{1}, R_{2}, \gamma\right) & =\sum_{i j k} c_{i j k} \varphi_{i}\left(R_{2}\right) \varphi_{j}\left(R_{1}\right) \xi_{k}(\gamma) \\
& =\sum_{i j k} c_{j i k} \varphi_{i}\left(R_{1}\right) \varphi_{j}\left(R_{2}\right) \xi_{k}(\gamma)
\end{aligned}
$$

since $\xi_{k}(\gamma)$ is composed of Legendre polynomials which are even in $\gamma$. Therefore,

$$
c_{i j k}= \pm c_{j i k}
$$

where the upper "+" state corresponds to singlet scattering and the lower "-" state corresponds to triplet scattering. It is then possible to truncate the basis set to give

$$
\Psi_{s}\left(R_{1}, R_{2}, \gamma\right)=\sum_{k} \sum_{i \geq j} c_{i j k}\left[\varphi_{i}\left(R_{1}\right) \varphi_{j}\left(R_{2}\right) \pm \varphi_{j}\left(R_{1}\right) \varphi_{i}\left(R_{2}\right)\right] \xi_{k}(\gamma)
$$

where the subscript $s$ indicates symmetrization of the function. The expansion coefficients $c_{i j k}$ are chosen so as to ensure orthonormality of the three dimensional basis functions. For singlet scattering the spatial $L^{2}$ basis functions are (in bra/ket notation)

$$
|i, j, k\rangle_{s}= \begin{cases}\varphi_{i}\left(R_{1}\right) \varphi_{j}\left(R_{2}\right) \xi_{k}(\gamma) & \text { if } i=j \\ \frac{1}{\sqrt{2}}\left[\varphi_{i}\left(R_{1}\right) \varphi_{j}\left(R_{2}\right)+\varphi_{j}\left(R_{1}\right) \varphi_{i}\left(R_{2}\right)\right] \xi_{k}(\gamma) & \text { if } i>j\end{cases}
$$

where the order of indices in the ket determines the spatial independent variable. For the triplet case the only form is

$$
|i, j, k\rangle_{s}=\frac{1}{\sqrt{2}}\left[\varphi_{i}\left(R_{1}\right) \varphi_{j}\left(R_{2}\right)-\varphi_{j}\left(R_{1}\right) \varphi_{i}\left(R_{2}\right)\right] \xi_{k}(\gamma)
$$


The kinetic energy in this basis is diagonal in the angular degree of freedom:

$$
{ }_{s}\left\langle i j k\left|\hat{T}_{1}+\hat{T}_{2}\right| i^{\prime} j^{\prime} k^{\prime}\right\rangle_{s}={ }_{s}\left\langle i j\left|\hat{T}_{1}+\hat{T}_{2}\right| i^{\prime} j^{\prime}\right\rangle_{s} \delta_{k k^{\prime}}
$$

Utilizing the orthogonality of the one-dimensional DVR functions gives

$$
\begin{aligned}
{ }_{s}\left\langle i j k\left|\hat{T}_{1}+\hat{T}_{2}\right| i^{\prime} j^{\prime} k^{\prime}\right\rangle_{s}= & \left(t_{i i^{\prime}} \delta_{j j^{\prime}}+t_{j j^{\prime}} \delta_{i i^{\prime}} \pm t_{j i^{\prime}} \delta_{i j^{\prime}} \pm t_{i j^{\prime}} \delta_{j i^{\prime}}\right) \delta_{k k^{\prime}} \\
& \text { if } i>j \text { and } i^{\prime}>j^{\prime} \\
{ }_{s}\left\langle i i k\left|\hat{T}_{1}+\hat{T}_{2}\right| i^{\prime} j^{\prime} k^{\prime}\right\rangle_{s}= & \sqrt{2}\left(t_{i i^{\prime}} \delta_{i j^{\prime}}+t_{i j^{\prime}} \delta_{i i^{\prime}}\right) \delta_{k k^{\prime}} \\
& \text { if } i=j \text { and } i^{\prime}>j^{\prime}, \text { and } \\
{ }_{s}\left\langle i i k\left|\hat{T}_{1}+\hat{T}_{2}\right| i^{\prime} i^{\prime} k^{\prime}\right\rangle_{s}= & 2 t_{i i^{\prime}} \delta_{i i^{\prime}} \delta_{k k^{\prime}} \\
& \text { if } i=j \text { and } i^{\prime}=j^{\prime}
\end{aligned}
$$

where $t_{i j^{\prime}}$ is the one-dimensional radial kinetic energy matrix for the DVR $\left\{\varphi_{i}\right\}$ basis set. The orbital angular momentum terms in the Hamiltonian are given by

$$
\begin{aligned}
{ }_{s}\left\langle i j k\left|\frac{1}{2}\left(\frac{1}{R_{1}^{2}}+\frac{1}{R_{2}^{2}}\right) \hat{L}^{2}\right| i^{\prime} j^{\prime} k^{\prime}\right\rangle_{s}= & \frac{1}{2} t_{k k^{\prime}}\left(\frac{1}{R_{i}^{2}}+\frac{1}{R_{j}^{2}}\right) \delta_{i i^{\prime}} \delta_{j j^{\prime}} \\
& \text { if } i>j \text { and } i^{\prime}>j^{\prime} \\
{ }_{s}\left\langle i i k\left|\frac{1}{2}\left(\frac{1}{R_{1}^{2}}+\frac{1}{R_{2}^{2}}\right) \hat{L}^{2}\right| i^{\prime} j^{\prime} k^{\prime}\right\rangle_{s}= & 0 \\
& \text { if } i=j \text { and } i^{\prime}>j^{\prime}, \text { and } \\
{ }_{s}\left\langle i i k\left|\frac{1}{2}\left(\frac{1}{R_{1}^{2}}+\frac{1}{R_{2}^{2}}\right) \hat{L}^{2}\right| i^{\prime} i^{\prime} k^{\prime}\right\rangle_{s}= & \frac{1}{R_{i}^{2}} t_{k k^{\prime}} \delta_{i i^{\prime}} \\
& \text { if } i=j \text { and } i^{\prime}=j^{\prime}
\end{aligned}
$$

where $t_{i j^{\prime}}$ is the one-dimensional angular kinetic energy matrix for the DVR $\left\{\xi_{i}\right\}$ basis set. The potential energy and overlap matrices have the following simple form:

$$
\begin{aligned}
{ }_{s}\left\langle i j k|\hat{V}| i^{\prime} j^{\prime} k^{\prime}\right\rangle_{s} & =V_{i j k} \delta_{i i^{\prime}} \delta_{j j^{\prime}} \delta_{k k^{\prime}} \\
{ }_{s}\left\langle i j k|\hat{O}| i^{\prime} j^{\prime} k^{\prime}\right\rangle_{s} & =\delta_{i i^{\prime}} \delta_{j j^{\prime}} \delta_{k k^{\prime}}
\end{aligned}
$$

where

$$
V_{i j k}=-\frac{1}{R_{i}}-\frac{1}{R_{j}}+\left(R_{i}^{2}+R_{j}^{2}-2 R_{i} R_{j} \cos \gamma_{k}\right)^{-1 / 2}
$$


where $R_{i}$ is the $i^{\text {th }}$ eigenvalue obtained in the diagonalization of $\hat{\mathbf{x}}^{(2)}$ defined in (3.52). Combining all terms of the Hamiltonian together results in the following functional forms. First, in the singlet $(S=0)$ case

$$
\begin{aligned}
H_{i j k i^{\prime} j^{\prime} k^{\prime}}= & \left(t_{i i^{\prime}} \delta_{j j^{\prime}}+t_{j j^{\prime}} \delta_{i i^{\prime}}+t_{j i^{\prime}} \delta_{i j^{\prime}}+t_{i j^{\prime}} \delta_{j i^{\prime}}\right) \delta_{k k^{\prime}}+ \\
& \frac{1}{2} t_{k k^{\prime}}\left(\frac{1}{R_{i}^{2}}+\frac{1}{R_{j}^{2}}\right) \delta_{i i^{\prime}} \delta_{j j^{\prime}}+V_{i j k} \delta_{i i^{\prime}} \delta_{j j^{\prime}} \delta_{k k^{\prime}} \\
& \text { if } i>j \text { and } i^{\prime}>j^{\prime}, \\
H_{i j k i^{\prime} j^{\prime} k^{\prime}}= & \sqrt{2}\left(t_{i i^{\prime}} \delta_{i j^{\prime}}+t_{i j^{\prime}} \delta_{i i^{\prime}}\right) \delta_{k k^{\prime}} \\
& \text { if } i=j \text { and } i^{\prime}>j^{\prime}, \\
H_{i j k k^{\prime} j^{\prime} k^{\prime}}= & \sqrt{2}\left(t_{i i^{\prime}} \delta_{j i^{\prime}}+t_{j i^{\prime}} \delta_{i i^{\prime}}\right) \delta_{k k^{\prime}} \\
& \text { if } i>j \text { and } i^{\prime}=j^{\prime}, \text { and } \\
H_{i j k k^{\prime} j^{\prime} k^{\prime}}= & 2 t_{i i^{\prime}} \delta_{i i^{\prime}} \delta_{k k^{\prime}}+t_{k k^{\prime}}\left(\frac{1}{R_{i}^{2}}\right) \delta_{i i^{\prime}}+V_{i i k} \delta_{i i^{\prime}} \delta_{k k^{\prime}} \\
& \text { if } i=j \text { and } i^{\prime}=j^{\prime} .
\end{aligned}
$$

And in the triplet $(S=1)$ case

$$
\begin{aligned}
H_{i j k i^{\prime} j^{\prime} k^{\prime}}= & \left(t_{i i^{\prime}} \delta_{j j^{\prime}}+t_{j j^{\prime}} \delta_{i i^{\prime}}+t_{j i^{\prime}} \delta_{i j^{\prime}}+t_{i j^{\prime}} \delta_{j i^{\prime}}\right) \delta_{k k^{\prime}}+ \\
& \frac{1}{2} t_{k k^{\prime}}\left(\frac{1}{R_{i}^{2}}+\frac{1}{R_{j}^{2}}\right) \delta_{i i^{\prime}} \delta_{j j^{\prime}}+V_{i j k} \delta_{i i^{\prime}} \delta_{j j^{\prime}} \delta_{k k^{\prime}} \\
& \text { if } i>j \text { and } i^{\prime}>j^{\prime} .
\end{aligned}
$$

The sparse nature of the Hamiltonian matrix is readily apparent from these formulae.

\subsection{Representation of the Free Functions}

\subsubsection{Background}

The form of the so-called "free" functions used in this study is of crucial importance in contributing to the success of the calculation. These functions were roughly described previously in terms of their asymptotic behavior.

$$
\begin{aligned}
& \Psi_{0 \mathbf{n}}\left(R_{1}, R_{2}, \gamma\right) \sim k_{\mathbf{n}}^{-1 / 2} e^{-i\left[k_{\mathbf{n}} R_{1}-\left(\pi l_{\mathbf{n}} / 2\right)\right]} \phi_{\mathbf{n}}\left(R_{2}, \gamma\right) \quad \text { as } R_{1} \rightarrow+\infty \\
& \Psi_{1 \mathbf{n}}\left(R_{1}, R_{2}, \gamma\right) \sim k_{\mathbf{n}}{ }^{-1 / 2} e^{i\left[k_{\mathbf{n}} R_{1}-\left(\pi l_{\mathbf{n}} / 2\right)\right]} \phi_{\mathbf{n}}\left(R_{2}, \gamma\right)
\end{aligned}
$$


Since the region near $R=0$ is within the interaction region (and therefore is primarily the domain of the $L^{2}$ DVR basis) the boundary condition applied to $\Psi_{\text {on }}$ and $\Psi_{1 \mathrm{n}}$ here is considerably less restrictive than that in the asymptotic region, mainly because the $L^{2}$ DVR basis can in some sense "pick up the slack". Ordinarily in a simple coupledchannel expansion one would impose the boundary condition

$$
\Psi_{\text {on }}\left(R_{1}, R_{2}, \gamma\right) \sim R_{1}^{l_{n}+1} \text { as } R_{1} \rightarrow 0
$$

due to the presence of a centrifugal barrier. But in this study the wavefunction is actually represented by a linear combination of the free function and radial DVR functions multiplied by the bound state. As a result, as long as the free function $\Psi_{\text {on }}\left(R_{1}, R_{2}, \gamma\right)$ approaches zero rapidly enough at the origin that the resulting freefree matrix elements remain finite, this will be an adequate constraint since the radial DVR functions will provide the proper rectification of the boundary condition. As will be seen later, the introduction of a multiplicative cutoff function into the free function (in order to cause the free-free exchange matrix elements to vanish) will cause the free function to actually vanish within a finite distance from the nucleus anyway.

On the face of it, these boundary conditions appear to permit considerable freedom of choice in deciding upon the actual functional form of the free functions. But the computational reality of the situation is quite a bit less forgiving. The separation of the total wavefunction into that part existing inside the interaction region and that part existing outside requires that the $L^{2}$ DVR basis functions must provide finite particle density in any region of the scattering coordinate at which the free functions do not satisfy the Schrödinger equation. The free functions chosen should therefore solve the applicable Schrödinger equation as close to $R=0$ as possible in order to minimize the extent of the scattering coordinate which must be spanned by the $L^{2}$ functions. On the other hand, an exact solution of the Schrödinger equation is likely to have a complicated analytical form (or none at all in the case of a purely numerical solution) and therefore makes the calculation of free-free and bound-free integrals more computationally time-consuming. A balanced approach must therefore be reached. 
Ideally it would have been convenient if it were possible to use the free particle wavefunction associated with the proper centrifugal barrier and regularized at $R_{1}=0$.

$$
\Psi_{\text {on }}\left(R_{1}, R_{2}, \gamma\right)=f\left(R_{1}\right) k_{\mathbf{n}}^{-1 / 2} e^{-i\left[k_{\mathbf{n}} R_{1}-\left(\pi l_{\mathbf{n}} / 2\right)\right]} \phi_{\mathbf{n}}\left(R_{2}, \gamma\right)
$$

where $f\left(R_{1}\right)$ is a smooth cutoff function designed to keep $\Psi_{0 n}\left(R_{1}, R_{2}, \gamma\right)$ regular at the origin and has the asymptotic form

$$
f\left(R_{1}\right) \sim\left\{\begin{array}{ll}
0 & \text { as } R_{1} \rightarrow 0, \text { and } \\
1 & \text { as } R_{1} \rightarrow \infty
\end{array} .\right.
$$

This choice of free function has been used with success by Colbert and Miller [41] in the molecular scattering system $\mathrm{H}+\mathrm{H}_{2}$ utilizing a DVR with the S matrix formulation of the Kohn variational principle.

A better approximation to the free function is that of a spherical Hankel function [37, p. 437] multiplied by a regularizing cutoff function,

$$
\Psi_{\text {on }}\left(R_{1}, R_{2}, \gamma\right)=-f\left(R_{1}\right) i k_{\mathbf{n}} R_{1} h_{l_{\mathbf{n}}}^{(2)}\left(k_{\mathbf{n}} R_{1}\right) \phi_{\mathbf{n}}\left(R_{2}, \gamma\right)
$$

In this case the cutoff function must be strong enough at the origin to regularize the divergent Neumann solution contained in the spherical Hankel function. This function has been used successfully by Zhang and Miller in the $F+H_{2}$ [52] and $D+H_{2}$ [54] scattering systems.

Unfortunately, the strengths of the interactions between electrons and target atoms are larger than those present in typical atom-molecule scattering systems. For atoms scattering from diatomic molecules with no permanent dipole moment, the strongest long range forces are the so-called dispersion forces [83, p. 103] which have the asymptotic form

$$
V(R) \sim-R^{-6}
$$

In contrast, electron-atom scattering calculations must contend with the net charge present on the electron together with the polarizability of the target atom, resulting in much longer range interactions which prevent the use of simple complex exponentials and spherical Hankel functions as free functions. The actual analytical form for the 
asymptotic wavefunction is discussed in the literature [84] and in standard textbooks $[4$, p. 160].

In order to more clearly see the source of this difficulty, the coupled-channel equations giving the form of the asymptotic wavefunction outside the exchange region for the scattering of a low energy electron from a hydrogen atom $(J=0)$ are given as

$$
\left[-\frac{1}{2} \frac{d^{2}}{d R_{1}^{2}}+\frac{l_{i}\left(l_{i}+1\right)}{2 R_{1}^{2}}-\frac{1}{R_{1}}-\frac{k_{i}^{2}}{2}\right] F_{\mathbf{n} i}\left(R_{1}\right)+\sum_{j}\left[F_{\mathbf{n} j}\left(R_{1}\right)\left\langle\phi_{i}\left|\frac{1}{\left|\mathbf{R}_{1}-\mathbf{R}_{2}\right|}\right| \phi_{j}\right\rangle{ }_{R_{2} \gamma}\right]=0
$$

where the total wavefunction is expanded as

$$
\Psi_{0 \mathbf{n}}\left(R_{1}, R_{2}, \gamma\right)=\sum_{i} F_{\mathbf{n} i}\left(R_{1}\right) \phi_{i}\left(R_{2}, \gamma\right)
$$

where $\mathbf{n}$ indicates the asymptotic quantum state index, $k_{i}^{2}$ is the asymptotic kinetic energy associated with the $i^{\text {th }}$ channel, and the subscripts $R_{2}$ and $\gamma$ on the "ket" indicate that the integration is only performed over those variables. Using the multipole expansion in (3.26) and combining the $l=0$ term of the summation with the electron-nuclear interaction potential gives

$$
\frac{d^{2}}{d R_{1}^{2}} F_{\mathbf{n} i}\left(R_{1}\right)=\sum_{j} V_{i j}\left(R_{1}\right) F_{\mathbf{n} j}\left(R_{1}\right)
$$

where

$$
V_{i j}\left(R_{1}\right)=\left[-k_{i}^{2}+\frac{l_{i}\left(l_{i}+1\right)}{R_{1}^{2}}\right] \delta_{i j}+\sum_{l=1}^{\infty} \frac{a_{i j l}}{R_{1}^{l+1}}
$$

and

$$
a_{i j l}=2\left\langle\phi_{i}^{r}\left|R^{l}\right| \phi_{j}^{r}\right\rangle \frac{\sqrt{\left(2 l_{i}+1\right)\left(2 l_{j}+1\right)}}{2 l+1}\left[C\left(l_{i} l_{j} l ; 000\right)\right]^{2} \text {. }
$$

Here, the superscript " $r$ " on the $\phi$ functions signifies that only the radial portion of the bound function is present. The $C\left(l_{i} l_{j} l ; 000\right)$ factor is the usual Clebsch-Gordan coefficient defined using the convention of Rose [85, p. 39]. A cursory examination of the definition of the $a_{i j l}$ term reveals that it is finite whenever $l_{i}, l_{j}$, and $l$ satisfy the triangle inequality

$$
\left|l_{i}-l_{j}\right| \leq l \leq l_{i}+l_{j}
$$

If only spherically symmetric bound states are included in the coupled-channel expansion, $l_{i}=0$ and $l_{j}=0$. There is then no $R_{1}$ dependence in the potential coupling 
term $V_{i j}\left(R_{1}\right)$. But when non-spherically symmetric bound states are included in the expansion, the potential coupling term acquires a strong $R^{-2}$ component which acts to alter the centrifugal potential in the second-order differential equation which the free functions satisfy. Use of the spherical Hankel functions as free functions for such a scattering calculation would cause the region of the scattering coordinate which must be covered by the $L^{2}$ DVR functions to be prohibitively large. It is therfore necessary to either make a more judicious choice of free function, or alter the $L^{2}$ basis in order to keep the calculation tractable.

Various methods of dealing with this difficulty have been reviewed [12, pp. 116-7] in the literature. For scattering above the $n=2$ threshold Seiler et al. [86] and Callaway and Wooten [87] included as part of the $L^{2}$ basis set functions of the form

$$
\left(1-e^{-\beta R}\right)^{l+2} \frac{1}{R^{2}} \sin \left(k_{\mathbf{n}} R\right) \text { and }\left(1-e^{-\beta R}\right)^{l+2} \frac{1}{R^{2}} \cos \left(k_{\mathbf{n}} R\right) .
$$

where the $\left(1-e^{-\beta R}\right)^{l+2}$ factor was included to provide adequate regularization of the functions at the origin. Although the inclusion of these functions causes an improvement in the performance of the variational calculation, it has the disadvantage that the $L^{2}$ basis is energy-dependent and therefore requires that the bound-bound Hamiltonian must be recalculated at each scattering energy.

Oberoi and Nesbet [88] have used a more effective means of including the long-range potential terms in the form of their free functions. The scattering coordinate is divided into two regions, $R>R_{0}$ and $R<R_{0}$, where $R_{0}$ is a demarcation point outside the exchange region of the scattering system. In the $R>R_{0}$ region the non-local operators present in the Hamiltonian (resulting from the imposition of proper electron wavefunction symmetry) do not manifest themselves and can therefore be ignored. The resulting simplified coupled differential equations can be solved numerically by various techniques, such as that of Burke and Schey [89]. Since these solutions will in general not have the correct asymptotic behavior at $R=0$, they are fitted at $R=R_{0}$ to two functions which do have the correct asymptotic behavior at $R=0$. The two coefficients on these functions are supplied by matching the functions and their first derivatives at $R=R_{0}$. The functional form of the free function 
components is then

$$
F_{\mathbf{n} i}(R)= \begin{cases}c_{\mathbf{n} i 1} h_{1}(R)+c_{\mathbf{n} i 2} h_{2}(R) & \text { for } R<R_{0} \\ H_{\mathbf{n} i}(R) & \text { for } R>R_{0}\end{cases}
$$

The only restrictions on the two functions $h_{1}$ and $h_{2}$ are that they be linearly independent and regular at the origin. The integrations of the free-free and bound-free integrals arising in the Kohn variational principle are performed over the finite interval $\left(0, R_{0}\right)$ since the components of the free function outside this region exactly solve the coupled-channel equations.

\subsubsection{Derivation of the Free Functions}

In the present work, a variation of this latter technique is utilized which enables one to exercise more freedom in choosing the functional form of the free function. The free function components outside the interval $\left(0, R_{0}\right)$ are assumed to take the form of an expansion in reciprocal powers of $R$ as originally done by Burke and Schey.

$$
F_{\mathbf{n} i}(R)=\sum_{\kappa=1}^{c_{0}} \sum_{p=0}^{p_{\max }}\left[\alpha_{p}^{\mathbf{n} i \kappa} \sin \left(k_{\kappa} R\right)+\beta_{p}^{\mathbf{n} i \kappa} \cos \left(k_{\kappa} R\right)\right] R^{-p}
$$

where $\mathbf{n}$ is the composite open channel index associated with the free function, $i$ is the bound state index, $c_{o}$ is the total number of open channels present, and $k_{\kappa}$ is the magnitude of the asymptotic wave vector for the $\kappa^{\text {th }}$ open channel. Substitution of this functional form into (3.97) and equating coefficients of $R^{-p} \sin \left(k_{\kappa} R\right)$ and $R^{-p} \cos \left(k_{\kappa} R\right)$ gives a set of recurrence relations for the $\alpha_{p}^{\text {ni }}$ and $\beta_{p}^{\text {nik }}$ expansion coefficients.

$$
\begin{aligned}
\left(k_{i}^{2}-k_{\kappa}^{2}\right) \alpha_{p}^{\mathbf{n} i \kappa}= & -2 k_{\kappa}(p-1) \beta_{p-1}^{\mathbf{n} i \kappa}+\left[l_{i}\left(l_{i}+1\right)-(p-1)(p-2)\right] \alpha_{p-2}^{\mathbf{n} i \kappa}+ \\
& \sum_{j=1}^{c_{o}} \sum_{l=1}^{\infty} a_{i j l} \alpha_{p-l-1}^{\mathbf{n} j \kappa} \\
\left(k_{i}^{2}-k_{\kappa}^{2}\right) \beta_{p}^{\mathbf{n} i \kappa}= & 2 k_{\kappa}(p-1) \alpha_{p-1}^{\mathbf{n} i \kappa}+\left[l_{i}\left(l_{i}+1\right)-(p-1)(p-2)\right] \beta_{p-2}^{\mathbf{n} i \kappa}+ \\
& \sum_{j=1}^{c_{o}} \sum_{l=1}^{\infty} a_{i j l} \beta_{p-l-1}^{\mathbf{n} j \kappa}
\end{aligned}
$$

It will be noticed that Burke and Schey's corresponding formulae do not have the extra $l_{i}\left(l_{i}+1\right)$ term present, probably because they absorbed the centrifugal potential into 
the associated $a_{i j l}$ factor. Adopting the hydrogen bound state indexing convention that

$$
\begin{aligned}
& \phi_{1}(R, \gamma)=\phi_{1 s}(R, \gamma) \\
& \phi_{2}(R, \gamma)=\phi_{2 s}(R, \gamma) \\
& \phi_{3}(R, \gamma)=\phi_{2 p}(R, \gamma)
\end{aligned}
$$

along with the formulae

$$
\begin{aligned}
\phi_{1 s}(R, \gamma) & =2 R e^{-R} \psi_{10}(\gamma) \\
\phi_{2 s}(R, \gamma) & =\frac{1}{2 \sqrt{2}} R(2-R) e^{-R / 2} \psi_{10}(\gamma) \\
\phi_{2 p}(R, \gamma) & =\frac{1}{2 \sqrt{6}} R^{2} e^{-R / 2} \psi_{20}(\gamma),
\end{aligned}
$$

where $\psi_{n 0}(\gamma)$ is defined by (3.56), one obtains by substitution into (3.99) that

$$
\begin{aligned}
& a_{11 l}=\frac{(l+2) !}{2^{l+1}} \\
& a_{12 l}=-\frac{1}{\sqrt{2}}\left(\frac{2}{3}\right)^{l+4} l(l+2) ! \\
& a_{13 l}=\frac{1}{\sqrt{6}}\left(\frac{2}{3}\right)^{l+4}(l+3) ! \\
& a_{22 l}=\frac{1}{8}\left(l^{2}+3 l+4\right)(l+2) ! \\
& a_{23 l}=-\frac{1}{8 \sqrt{3}}(l+2)(l+3) ! \\
& a_{33 l}=\frac{1}{24}(l+4) !
\end{aligned}
$$

Because of the $\left(k_{i}^{2}-k_{\kappa}^{2}\right)$ factor multiplying $\alpha_{p}^{\text {ni }}$ and $\beta_{p}^{\text {ni }}$ in the recurrence relations, the actual relations determining both of these quantities are different in the two cases where $k_{i}=k_{\kappa}$ and $k_{i} \neq k_{\kappa}$. In the case where $k_{i} \neq k_{\kappa}$,

$$
\begin{aligned}
\alpha_{p}^{\mathbf{n} i \kappa}= & \frac{1}{k_{i}^{2}-k_{\kappa}^{2}}\left\{-2 k_{\kappa}(p-1) \beta_{p-1}^{\mathbf{n} i \kappa}+\left[l_{i}\left(l_{i}+1\right)-(p-1)(p-2)\right] \alpha_{p-2}^{\mathbf{n} i \kappa}+\right. \\
& \left.\sum_{j=1}^{c_{0}} \sum_{l=1}^{\infty} a_{i j l} \alpha_{p-l-1}^{\mathbf{n} j \kappa}\right\}, \text { and } \\
\beta_{p}^{\mathbf{n} i \kappa}= & \frac{1}{k_{i}^{2}-k_{\kappa}^{2}}\left\{2 k_{\kappa}(p-1) \alpha_{p-1}^{\mathbf{n} i \kappa}+\left[l_{i}\left(l_{i}+1\right)-(p-1)(p-2)\right] \beta_{p-2}^{\mathbf{n} i \kappa}+\right.
\end{aligned}
$$




$$
\left.\sum_{j=1}^{c_{0}} \sum_{l=1}^{\infty} a_{i j l} \beta_{p-l-1}^{\mathbf{n} j \kappa}\right\}
$$

And when $k_{i}=k_{\kappa}$,

$$
\begin{aligned}
& \alpha_{p}^{\mathbf{n} i \kappa}=\frac{1}{2 k_{\kappa} p}\left\{\left[p(p-1)-l_{i}\left(l_{i}+1\right)\right] \beta_{p-1}^{\mathbf{n} i \kappa}-\sum_{j=1}^{c_{0}} \sum_{l=1}^{\infty} a_{i j l} \beta_{p-l}^{\mathbf{n j \kappa}}\right\}, \quad \text { and } \\
& \beta_{p}^{\mathbf{n} i \kappa}=\frac{-1}{2 k_{\kappa} p}\left\{\left[p(p-1)-l_{i}\left(l_{i}+1\right)\right] \alpha_{p-1}^{\mathbf{n} i \kappa}-\sum_{j=1}^{c_{0}} \sum_{l=1}^{\infty} a_{i j l} \alpha_{p-l}^{\mathbf{n} j \kappa}\right\}
\end{aligned}
$$

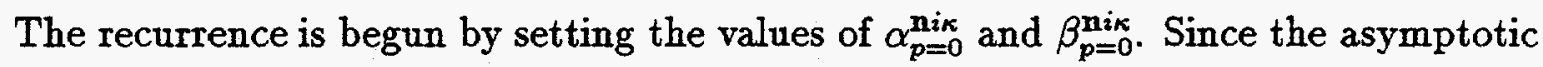
wavefunction is in a pure bound state only $\alpha_{0}^{\mathrm{n} n n}$ and $\beta_{0}^{\mathrm{nnn}}$ are finite. Combining (3.9), (3.103), and (3.96) one can derive the values of $\alpha_{0}^{\mathbf{n} n n}$ and $\beta_{0}^{\text {nnn }}$. Using the equation

$$
\alpha_{p}^{\mathbf{n} i \kappa} \sin k_{\kappa} R+\beta_{p}^{\mathbf{n} i \kappa} \cos k_{\kappa} R=\bar{\alpha}_{p}^{\mathbf{n} i \kappa} e^{-i\left(k_{\kappa} R-\frac{\pi l_{\mathbf{n}}}{2}\right)}+\bar{\beta}_{\boldsymbol{p}}^{\mathbf{n} i \kappa} e^{i\left(k_{\kappa} R-\frac{\pi l \mathbf{n}}{2}\right)}
$$

and the identities

$$
\sin u=\frac{1}{2 i}\left(e^{u}-e^{-u}\right) \quad \text { and } \quad \cos u=\frac{1}{2}\left(e^{u}+e^{-u}\right)
$$

the intialization conditions are derived as

$$
\begin{aligned}
& \alpha_{0}^{\mathbf{n} n n}=-i^{l \mathbf{n}+1} k_{\mathbf{n}}^{-1 / 2}, \\
& \beta_{0}^{\mathbf{n} n n}=i^{\ln } k_{\mathbf{n}}^{-1 / 2} .
\end{aligned}
$$

The recurrence is performed for $p=0,1,2,3, \ldots, p_{\max }$. Obviously, if $p_{\max }=0$ the expanded free function is then just a simple free particle wavefunction.

It is interesting to note that this recurrence procedure generates a free wavefunction which (assuming proper convergence of the expansion) could also be generated through the use of a conventional Numerov propagation algorithm in which the coupled-channel expansion in each of the three open channels is solved numerically in a step-wise fashion beginning in the asymptotic region of the scattering coordinate and proceding inward toward (but not into) the exchange region. In some sense, the present method can be thought of as an analytical Numerov algorithm which takes advantage of the simple analytical form of the potential energy function. 
The accuracy of successive approximations of the free function is demonstrated by examination of projections of $(\hat{H}-\hat{E}) \Psi_{0 n}\left(R_{1}, R_{2}, \gamma\right)$ onto each of the bound states $\phi_{i}\left(R_{2}, \gamma\right)$ included in the expansion used to solve the coupled-channel equations (3.97). This quantity is a function of the scattering coordinate $R_{1}$ and should vanish outside of the exchange region for the exact, physically correct wavefunction. Theoretically, the projection of $(\hat{H}-\hat{E}) \Psi_{0 n}$ onto any function (including closed channels and continuum functions) should vanish outside of the exchange region since $(\hat{H}-\hat{E}) \Psi_{\text {on }}$ vanishes for the correct $\Psi_{\text {on }}$.

Unfortunately, recurrence relations which result from inclusion of closed channels in the coupled-channel expansion [89] cannot be solved by setting their corresponding $p=0$ coefficients to a known value, since these decaying exponential components of the free function vanish as $R_{1} \rightarrow \infty$. Techniques for including closed channels in asymptotic expansions are available $[5,90,91,92]$, but in the present work the assumption is made that projections of $(\hat{H}-\hat{E}) \Psi_{0 n}$ onto the closed channel space are very small and are therefore safely ignored. Of course, this assumption breaks down when the scattering energy is just below the threshold for opening another channel, since the exponential tail for the $R_{1}$-dependent coefficient of that closed channel will be quite long and extend far beyond the exchange region.

Figures 3.2 through 3.10 show the previously described projections for a calculated free function which corresponds to an asymptotic $\phi_{2 s}$ state. Projections onto each of the three open channels $\phi_{1 s}, \phi_{2 s}$, and $\phi_{2 p}$ are shown as a function of different values of $p_{\max }$ for three different representative total energies - one just above the $n=2$ threshold, one just below the $n=3$ threshold, and one roughly in the middle of this energy region.

Clearly, the expansion works best at the energy just below the $n=3$ threshold, with rapid convergence for even small values of $R_{1}$. Of course, this behavior is deceptive since this is the energy region in which the omission of the $n=3$ closed channels begins to have a negative effect on the accuracy of the coupled-channel expansion. But the expansion is clearly strained at the energy just above the $n=2$ threshold, requiring a large value of $p_{\max }$ to converge the free function at even moderate values of $R_{1}$. This results from the inability of the sine and cosine expansion 


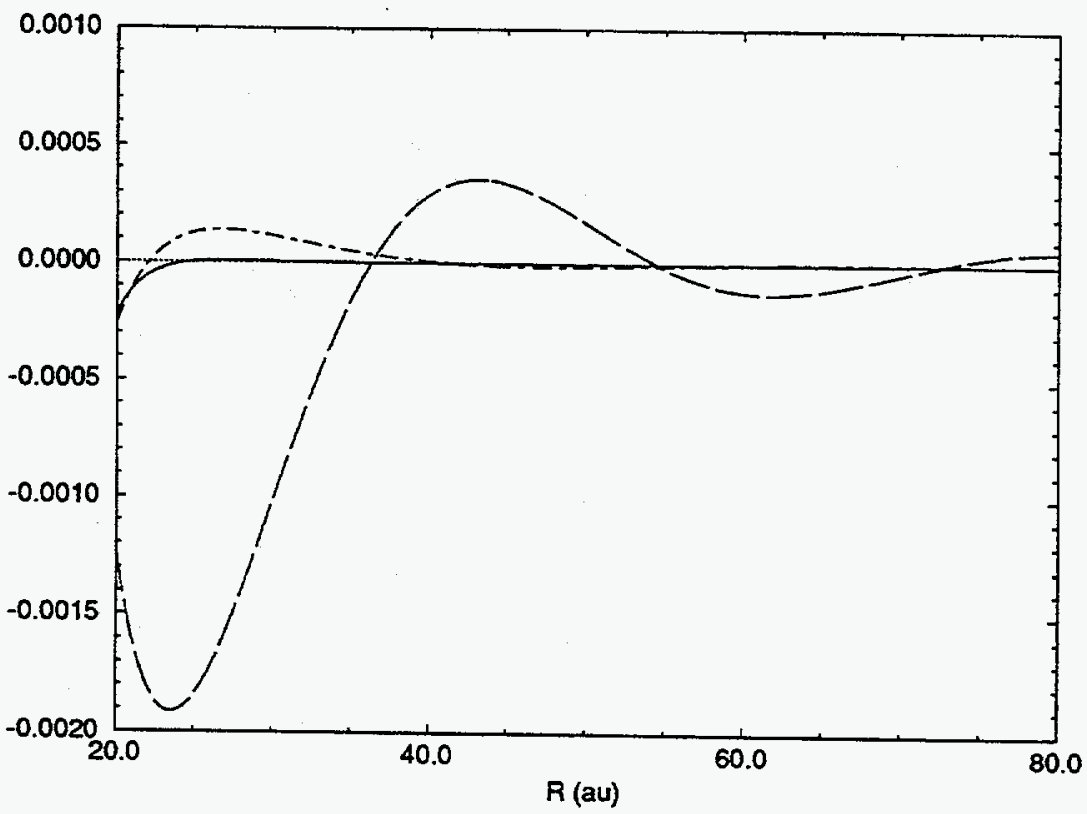

Figure 3.2: $\operatorname{Re}\left\langle\phi_{1 s}|\hat{H}-\hat{E}| \Psi_{0(2 s)}\right\rangle$ for $E=0.78$ Ryd. Dotted line: $p_{\max }=0$; Dashed line: $p_{\max }=1$; Long-dashed line: $p_{\max }=2$; Dot-dashed line: $p_{\max }=3$; Solid line: $p_{\max }=8$.

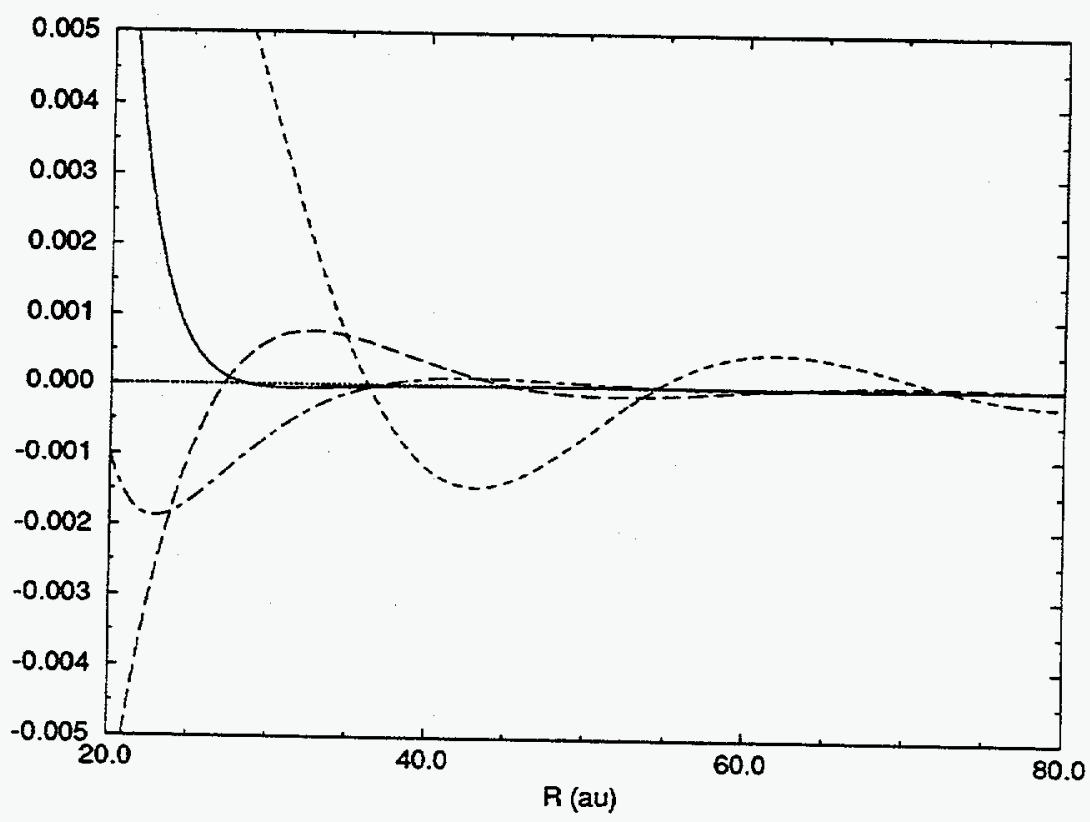

Figure 3.3: $\operatorname{Re}\left\langle\phi_{2 s}|\hat{H}-\hat{E}| \Psi_{0(2 s)}\right\rangle$ for $E=0.78$ Ryd. Dotted line: $p_{\max }=0$; Dashed line: $p_{\max }=1$; Long-dashed line: $p_{\max }=2$; Dot-dashed line: $p_{\max }=3$; Solid line: $p_{\max }=8$. 


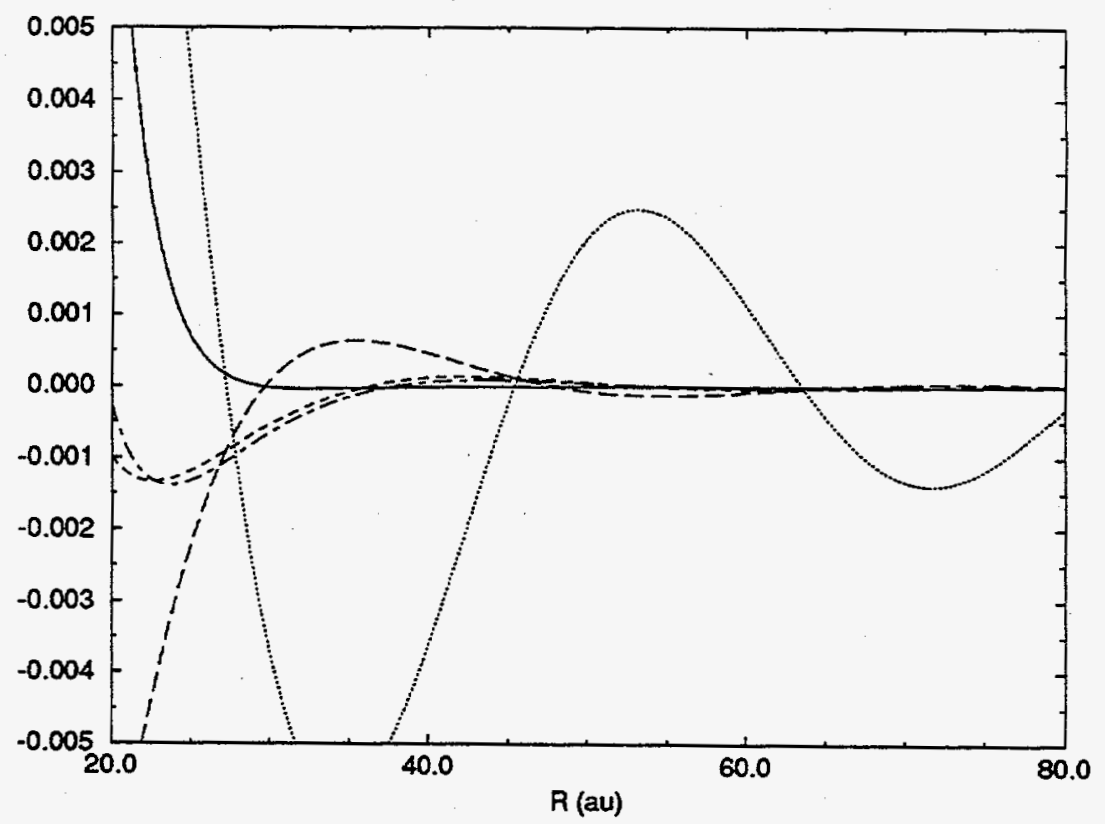

Figure 3.4: $\operatorname{Re}\left\langle\phi_{2 p}|\hat{H}-\hat{E}| \Psi_{0(2 s)}\right\rangle$ for $E=0.78$ Ryd. Dotted line: $p_{\max }=0$; Dashed line: $p_{\max }=1$; Long-dashed line: $p_{\max }=2$; Dot-dashed line: $p_{\max }=3$; Solid line: $p_{\max }=8$.

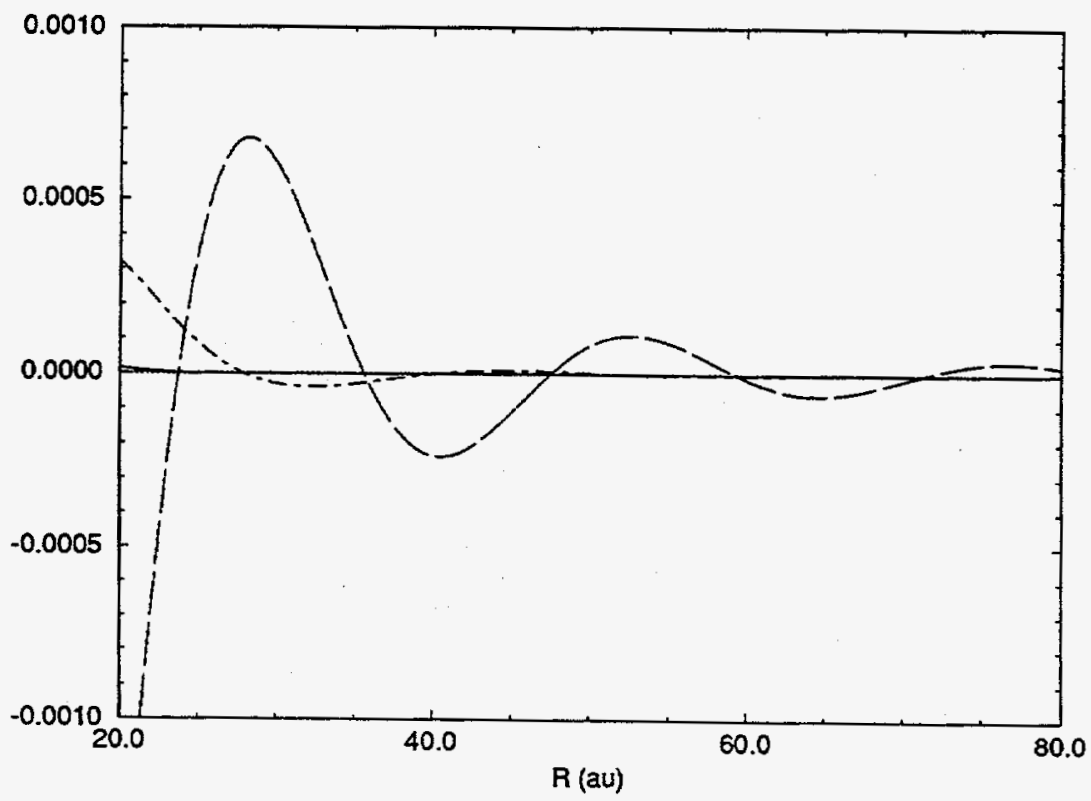

Figure 3.5: $\operatorname{Re}\left\langle\phi_{1 s}|\hat{H}-\hat{E}| \Psi_{0(2 s)}\right\rangle$ for $E=0.82$ Ryd. Dotted line: $p_{\max }=0$; Dashed line: $p_{\max }=1$; Long-dashed line: $p_{\max }=2$; Dot-dashed line: $p_{\max }=3$; Solid line: $p_{\max }=8$. 


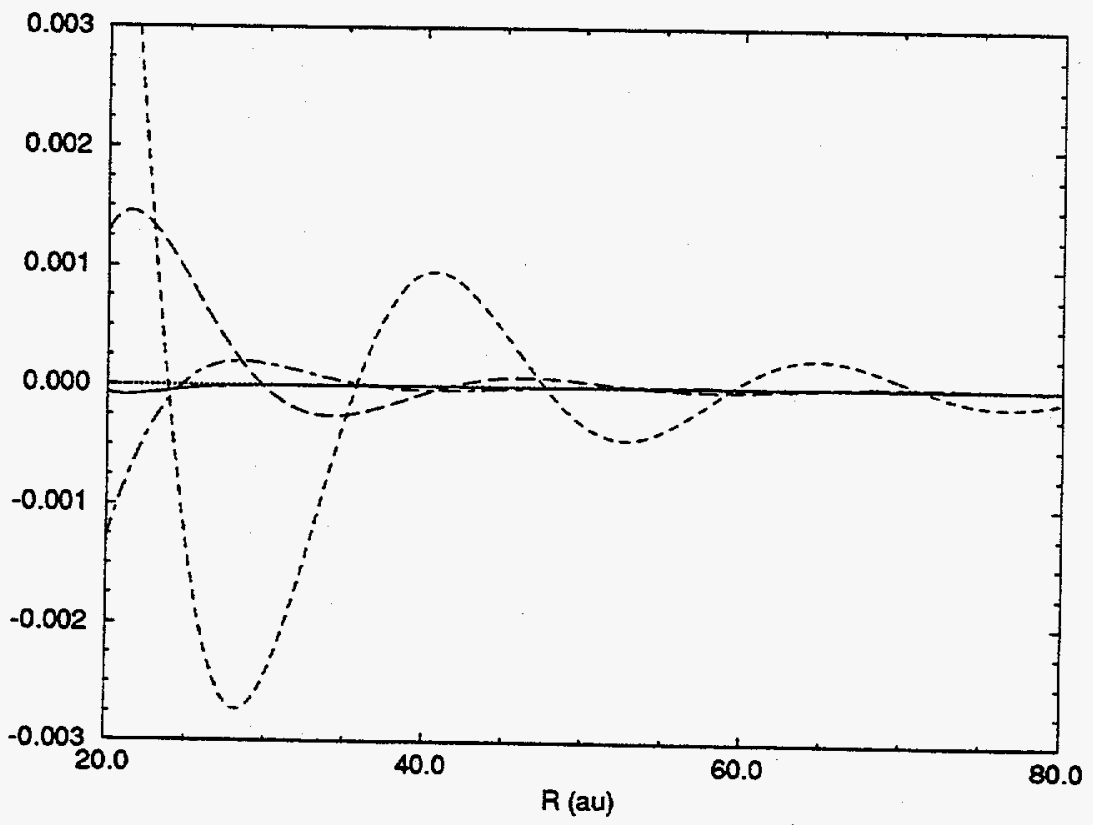

Figure 3.6: $\operatorname{Re}\left\langle\phi_{2 s}|\hat{H}-\hat{E}| \Psi_{0(2 s)}\right\rangle$ for $E=0.82$ Ryd. Dotted line: $p_{\max }=0$; Dashed line: $p_{\max }=1$; Long-dashed line: $p_{\max }=2$; Dot-dashed line: $p_{\max }=3$; Solid line: $p_{\max }=8$.

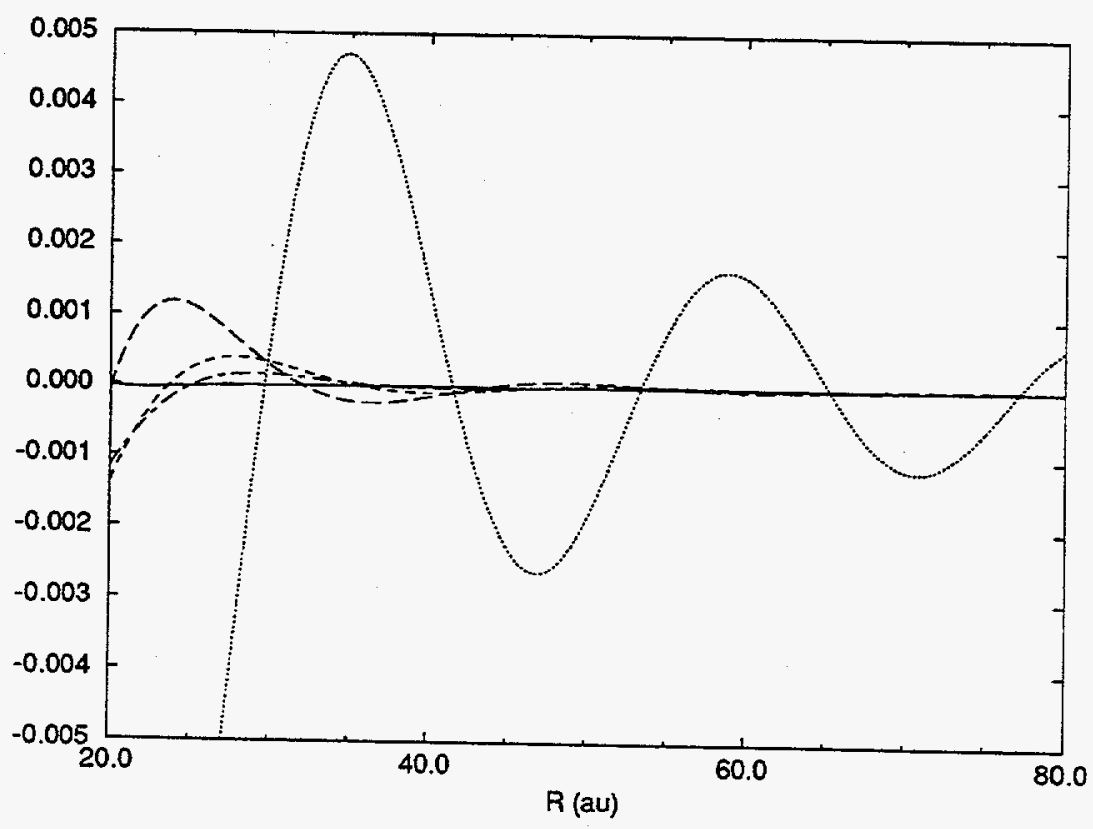

Figure 3.7: $\operatorname{Re}\left\langle\phi_{2 p}|\hat{H}-\hat{E}| \Psi_{(2 s)}\right\rangle$ for $E=0.82$ Ryd. Dotted line: $p_{\max }=0$; Dashed line: $p_{\max }=1$; Long-dashed line: $p_{\max }=2$; Dot-dashed line: $p_{\max }=3$; Solid line: $p_{\max }=8$. 


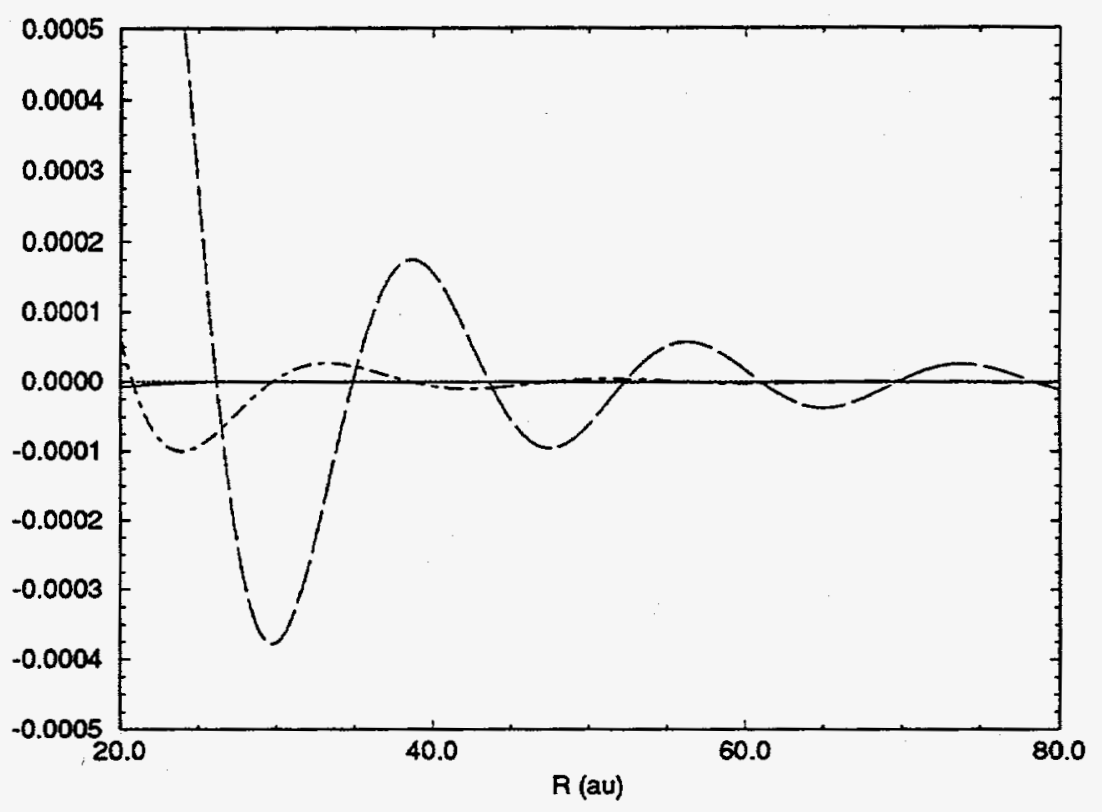

Figure 3.8: $\operatorname{Re}\left\langle\phi_{1 s}|\hat{H}-\hat{E}| \Psi_{0(2 s)}\right\rangle$ for $E=0.88 \mathrm{Ryd}$. Dotted line: $p_{\max }=0$; Dashed line: $p_{\max }=1$; Long-dashed line: $p_{\max }=2$; Dot-dashed line: $p_{\max }=3$; Solid line: $p_{\max }=8$.

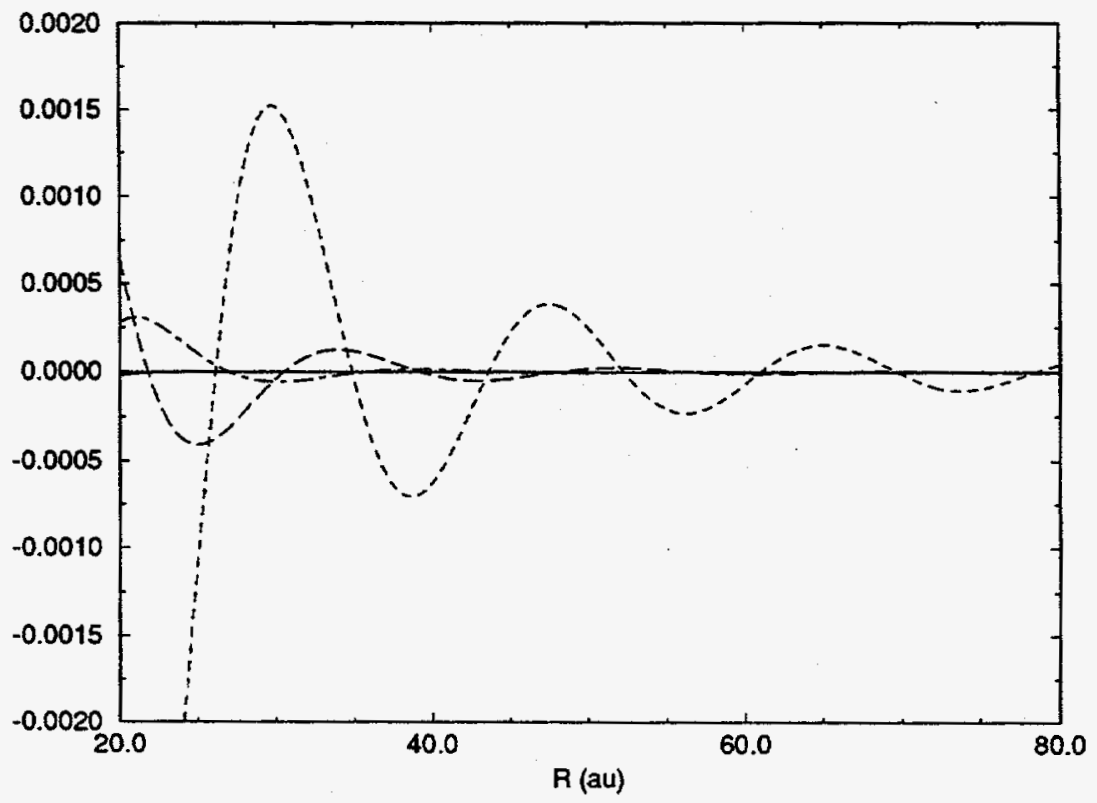

Figure 3.9: $\operatorname{Re}\left\langle\phi_{2 s}|\hat{H}-\hat{E}| \Psi_{0(2 s)}\right\rangle$ for $E=0.88 \mathrm{Ryd}$. Dotted line: $p_{\max }=0 ;$ Dashed line: $p_{\max }=1$; Long-dashed line: $p_{\max }=2$; Dot-dashed line: $p_{\max }=3$; Solid line: $p_{\max }=8$. 


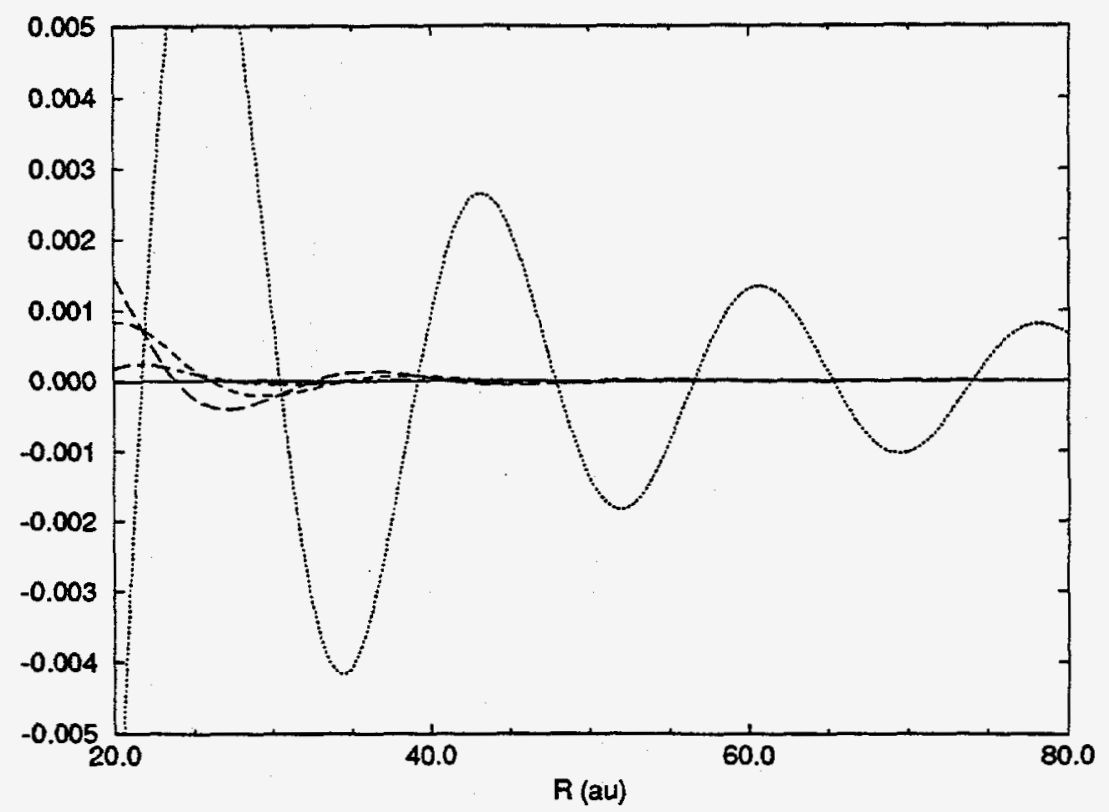

Figure 3.10: $\operatorname{Re}\left\langle\phi_{2 p}|\hat{H}-\hat{E}| \Psi_{0(2 s)}\right\rangle$ for $E=0.88$ Ryd. Dotted line: $p_{\max }=0$; Dashed line: $p_{\max }=1$; Long-dashed line: $p_{\max }=2$; Dot-dashed line: $p_{\max }=3$; Solid line: $p_{\max }=8$.

to represent a wavefunction with such low kinetic energy as is present just above an opened threshold. Not surprisingly, it is in the middle energy region between the $n=2$ and $n=3$ thresholds that the expansion seems to work best.

It is probably also instructive to examine how ineffective this expansion would be if only $\alpha_{p}^{\mathbf{n} i \kappa}$ and $\beta_{p}^{\mathbf{n} i \kappa}$ coefficients diagonal in $\mathbf{n}$ and $i$ were kept. (This would correspond to free functions which project only onto a single bound hydrogen state outside of the exchange region, a form of free function often used in molecular scattering calculations involving neutral species.) Figures 3.11 and 3.12 show components of the free function for three different energies between the $n=2$ and $n=3$ thresholds corresponding to asymptotic $\phi_{2 s}$ and $\phi_{2 p}$ states respectively where the asymptotic wavefunction is defined as before in (3.96).

$$
\Psi_{0 \mathbf{n}}\left(R_{1}, R_{2}, \gamma\right)=\sum_{i} F_{\mathbf{n} i}\left(R_{1}\right) \phi_{i}\left(R_{2}, \gamma\right)
$$

The $\phi_{2 s}$ and $\phi_{2 p}$ asymptotic states are shown here because these particular functions show the greatest amount of "mixing" far from the interaction region. 


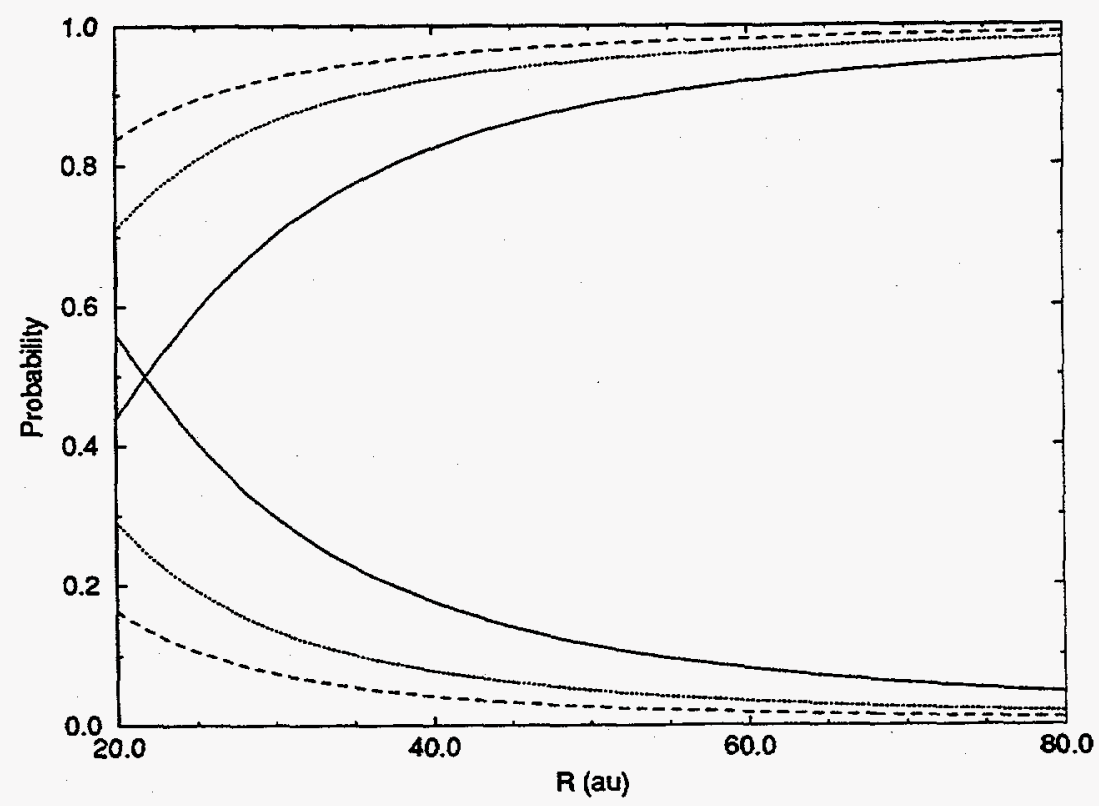

Figure 3.11: Mixing of $\mathrm{H}$ bound states for asymptotic $\phi_{2 s}$ state. $\left|F_{\mathbf{n}, i}\right|^{2} / \sum_{j}\left|F_{\mathbf{n}, j}\right|^{2}$ for $\mathbf{n}=\phi_{2 s}$ and $p_{\max }=8$. Solid line: $E=0.78 \mathrm{Ryd}$; Dotted line: $E=0.82 \mathrm{Ryd}$; Dashed line: $E=0.88$ Ryd. Curves approaching 1 asymptotically represent square moduli of $\phi_{2 s}$ coefficients. Curves approaching 0 asymptotically represent square moduli of $\phi_{2 p}$ coefficients. Square moduli of $\phi_{1 s}$ coefficients are very small and not visible on this scale.

Obviously, inclusion of the full compliment of bound states in the asymptotic expansion is required in order to accurately represent the asymptotic region (and therefore adequately reduce the size of the interaction region which must be spanned by the $L^{2}$ basis set.) This is particularly crucial in the case of the low kinetic energies associated with the $\phi_{2 s}$ and $\phi_{2 p}$ channels when the total energy is located just above the $n=2$ threshold.

While the behavior of this expansion in reciprocal powers of $R_{1}$ is quite adequate outside of the exchange region, it is considerably worse within the exchange region. This is not surprising since the derivation of the recurrence relations (3.104) and (3.105) assumed $R_{>}=R_{1}$ and $R_{<}=R_{2}$ prior to integrating out the $R_{2}$ and $\gamma$ degrees of freedom. One might assume that the inclusion of the cutoff function $f\left(R_{1}\right)$ previously described in (3.92) could be made sufficiently strong enough to ensure that the free function is well-behaved throughout the entire exchange region. Since the 


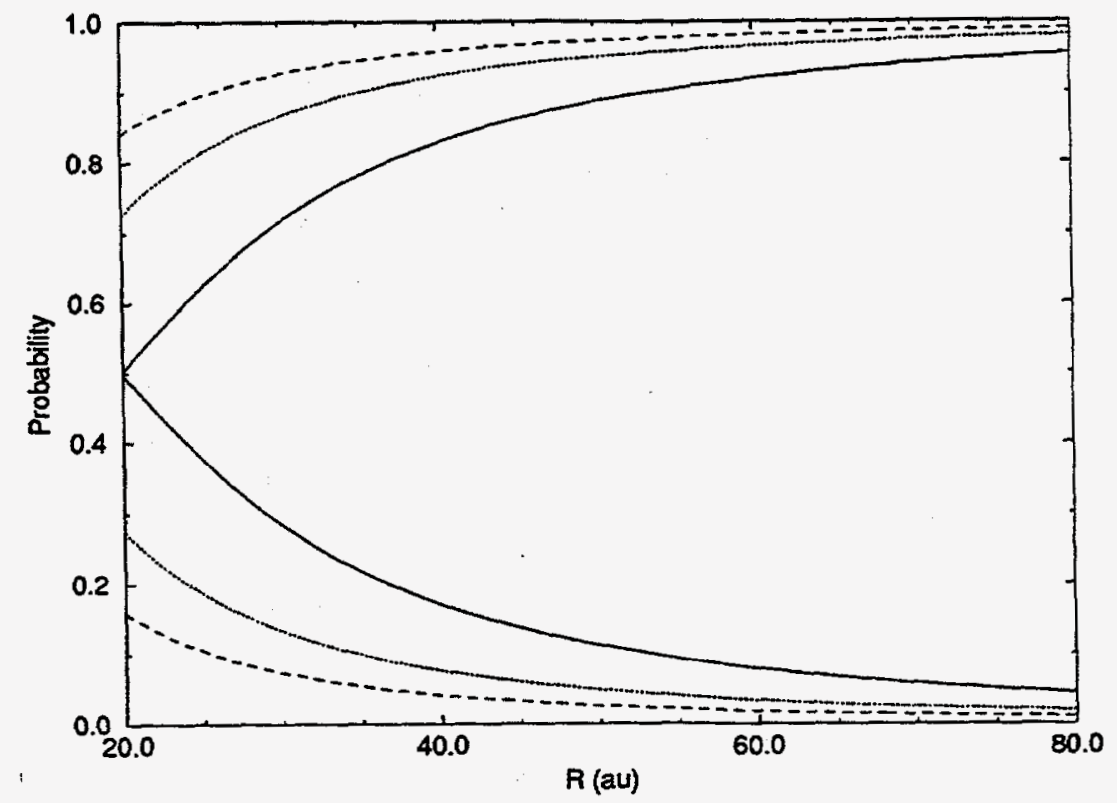

Figure 3.12: Mixing of $\mathrm{H}$ bound states for Asymptotic $\phi_{2 p}$ state. $\left|F_{\mathbf{n}, i}\right|^{2} / \sum_{j}\left|F_{\mathbf{n}, j}\right|^{2}$ for $\mathbf{n}=\phi_{2 p}$ and $p_{\max }=8$. Solid line: $E=0.78 \mathrm{Ryd}$; Dotted line: $E=0.82 \mathrm{Ryd}$; Dashed line: $E=0.88$ Ryd. Curves approaching 1 asymptotically represent square moduli of $\phi_{2 p}$ coefficients. Curves approaching 0 asymptotically represent square moduli of $\phi_{2 s}$ coefficients. Square moduli of $\phi_{1 s}$ coefficients are very small and not visible on this scale.

reciprocal $R_{1}$ expansion has only "removable" singularities, it is quite possible to find a large number of cutoff functions which would ensure regularity at the origin. The function

$$
f(R)=\left(1-e^{-\beta R}\right)^{l+2}
$$

used by Seiler et al. [86] and Callaway and Wooten [87] would suffice with a large enough value for $l$.

Unfortunately, relying solely on the cutoff function to ensure good behavior of the reciprocal $R_{1}$ expansion within the exchange region is not sufficient. This is because there continue to be parts of the exchange region in which the local kinetic energy of the free function is extremely high. Since these regions do not accurately reflect the correct physical situation within the exchange region, it is then up to the $L^{2}$ DVR functions to correct for this error. This would require a prohibitively large density of DVR points in this region, and it would be best to avoid such a requirement 
in the interest of reducing the size of the matrix inversion required in the calculation. As was mentioned previously, Oberoi and Nesbet [88] performed a spline fit of the asymptotic free function to a linear combination of two functions which have the proper boundary condition at the origin. A discussion of this method is provided just prior to (3.102). The method used in this treatment is very similar. But rather than restrict the spline fit to only two functions (and hence only allow a matching of $0^{\text {th }}$ and $1^{\text {st }}$ order derivatives at the demarcation parameter), a linear combination of four linearly-independent functions is used. This has the advantage of permitting a matching of $0^{\text {th }}, 1^{\text {st }}, 2^{\text {nd }}$, and $3^{\text {rd }}$ order derivatives, thereby permitting the use of simple automated integration routines in the calculation of the free-free matrix elements. The regions of integration then need not be split between exchange and non-exchange regions since the integrand (in spite of the presence of the $2^{\text {nd }}$ derivative kinetic energy operator) will be not only continuous, but will also have no cusps. This permits the efficient use of automated integration routines without the need of frequent function evaluations within "difficult" areas.

The choice of linearly-independent functions is now discussed. To allow maximum freedom of choice the requirement that these functions be regular at the origin is temporarily suspended. Instead, the entire free function (in both the $R_{1}>R_{0}$ and $R_{1}<R_{0}$ regions) is to be multiplied later by an external cutoff function which provides the proper regularization at the origin and also forces the exchange freefree matrix elements to vanish. (This aspect of the computation will be discussed later.) Certain characteristics of the four linearly-independent functions are desirable. When combined with the asymptotic free function they should emulate the correct local kinetic energy at the demarcation point $R_{0}$. The spline fit will ensure this. They should also be well-bounded within the exchange region in order to keep the coefficients of the $L^{2}$ DVR functions reasonably sized during the matrix inversion. They should be limited in bandwidth so that within any part of the exchange region which has a relatively low local kinetic energy it would not be necessary to provide a high density of DVR points to "undo the damage" done by the free function.

This suggests that a linear combination of sine and cosine functions would be most appealing. Choosing two frequencies is sufficient to determine the four linearly- 
independent functions. An examination of the following identities provides a clue in choosing the two frequencies.

$$
\begin{aligned}
& \sin x+\sin y=2 \sin \frac{1}{2}(x+y) \cos \frac{1}{2}(x-y) \\
& \cos x+\cos y=2 \cos \frac{1}{2}(x+y) \cos \frac{1}{2}(x-y)
\end{aligned}
$$

In each of these identities, when $x \approx y$ the righthand side of the equation is composed of a trigonometric function whose frequency is the average of $x$ and $y$, multiplied by a low-frequency cosine function which acts as a "modulation" envelope. Using this as a guide, the two frequencies needed to provide the two additional degrees of freedom needed in the spline fit are chosen such that they are nearly equal to each other and their average corresponds to the correct asymptotic frequency for the particular open channel being represented. The general form for the individual free function components within the exchange region is then taken to be

$$
F_{\mathbf{n} i}\left(R_{1}\right)=c_{\mathbf{n} i 1} \sin k_{a}\left(R_{1}\right)+c_{\mathbf{n} i 2} \cos k_{a}\left(R_{1}\right)+c_{\mathbf{n} i 3} \sin k_{b}\left(R_{1}\right)+c_{\mathbf{n} i 4} \cos k_{b}\left(R_{1}\right) .
$$

It is now necessary to determine the proper coefficients $c_{\text {nix }}$. The $0^{\text {th }}, 1^{\text {st }}$, $2^{\text {nd }}$, and $3^{\text {rd }}$ order derivatives of the asymptotic free function $F_{\mathbf{n} i}\left(R_{1}\right)$ are determined analytically from (3.103). These are matched to the corresponding derivatives of the free functions in the exchange region, resulting in the simultaneous equations

$$
\mathbf{A} \cdot \mathbf{c}=\mathbf{F}
$$

where

$$
\begin{aligned}
& \mathbf{A}=\left(\begin{array}{cccc}
\sin k_{a} R_{0} & \cos k_{a} R_{0} & \sin k_{b} R_{0} & \cos k_{b} R_{0} \\
k_{a} \cos k_{a} R_{0} & -k_{a} \sin k_{a} R_{0} & k_{b} \cos k_{b} R_{0} & -k_{b} \sin k_{b} R_{0} \\
-k_{a}^{2} \sin k_{a} R_{0} & -k_{a}^{2} \cos k_{a} R_{0} & -k_{a}^{2} \sin k_{b} R_{0} & -k_{a}^{2} \cos k_{b} R_{0} \\
-k_{a}^{3} \cos k_{a} R_{0} & k_{a}^{3} \sin k_{a} R_{0} & -k_{b}^{3} \cos k_{b} R_{0} & k_{b}^{3} \sin k_{b} R_{0}
\end{array}\right) \\
& \mathbf{c}=\left(\begin{array}{c}
c_{\mathbf{n} i 1} \\
c_{\mathbf{n} i 2} \\
c_{\mathbf{n} i 3} \\
c_{\mathbf{n} i 4}
\end{array}\right), \text { and }
\end{aligned}
$$




$$
\mathrm{F}=\left(\begin{array}{l}
F_{\mathrm{ni}}^{(0)}\left(R_{0}\right) \\
F_{\mathbf{n} i}^{(1)}\left(R_{0}\right) \\
F_{\mathbf{n} i}^{(2)}\left(R_{0}\right) \\
F_{\mathbf{n} i}^{(3)}\left(R_{0}\right)
\end{array}\right)
$$

Here, $k_{a} \approx k_{b}$ and $\frac{1}{2}\left(k_{a}+k_{b}\right)=k_{\mathbf{n}}$ where $\mathbf{n}$ is the $\mathbf{n}^{t h}$ open channel index. $F_{\mathbf{n} i}^{(j)}$ is the $j^{\text {th }}$ derivative of $F_{\mathbf{n} i}$.

The regularization of these "composite" free functions is accomplished by multiplying the entire function (in both the exchange and non-exchange regions) by a cutoff function $f(R)$. The function used for this purpose was developed by Groenenboom [93] and is defined by

$$
f(R)=\frac{1}{\sqrt{\pi}} \int_{-\infty}^{u} e^{-t^{2}} d t
$$

where

$$
\begin{aligned}
& u=x+C x^{3}, \quad \text { and } \\
& x=A(R-B) .
\end{aligned}
$$

$A, B$, and $C$ are parameters chosen to impart a physically reasonable character to the cutoff function, where $B$ is the center of the switching region, and $A$ and $C$ are strength parameters which determine the rate of switching. The final definition of $\Psi_{0 \mathrm{n}}$ is then given by (3.96). The use of the spline fit for the free function (rather than using the reciprocal $R_{1}$ expansion for the entire range of the scattering coordinate) allows maximum freedom in choosing the cutoff function parameters. Good use of this is made in the later discussion dealing with the computation of the free-free matrix elements. 


\subsection{Calculation of the Bound-Free Matrix Ele- ments}

The use of the $\mathrm{S}$ matrix Kohn variational principle requires the calculation of the bound-free matrix elements given by (3.11),

$$
\left(\mathbf{M}_{\mathbf{0}}\right)_{\mathrm{tn}}=\left\langle u_{\mathbf{t}}|\hat{H}-\hat{E}| \Psi_{\mathbf{0 n}}\right\rangle
$$

where as before $t$ is a three-dimensional composite DVR index and $\mathbf{n}$ is an open channel index. (For simplicity it is assumed in this section that the $L^{2}$ DVR functions $u_{\mathrm{t}}$ are not yet put in their symmetrized singlet and triplet irreducible representations.)

The procedure by which these matrix elements are calculated is now discussed. In the previous section, the method by which an analytical form for the free functions is calculated was described. The operation of the Hamiltonian given in (3.1) upon the free function is then a simple matter which can be performed analytically and inexpensively, especially in light of the simplicity of the potential energy function. Following this, the usual procedure used in calculating the bound-free matrix elements within a discrete variable representation involves explicitly calculating the quantity $(\hat{H}-\hat{E}) \Psi_{0 n}$ as a function of all the independent variables (in this case $R_{1}$, $R_{2}$, and $\gamma$ ) and multiplying this quantity by the corresponding quadrature weight factors which characterize the various DVR's used in each degree of freedom. So in the present system the bound-free matrix element would be given as

$$
\left(\mathbf{M}_{0}\right)_{\mathbf{t n}}=\sqrt{W_{\mathbf{t}}}\left\langle u_{\mathbf{t}}|\hat{H}-\hat{E}| \Psi_{\mathbf{0 n}}\right\rangle
$$

where

$$
\begin{aligned}
& W_{\mathbf{t}}=\omega_{1 t_{1}} \omega_{2 t_{2}} \omega_{3 t_{2}}, \quad \text { and } \\
& \left\langle u_{\mathbf{t}}\right|=\left\langle R_{1 t_{1}}, R_{2 t_{2}}, \gamma_{t_{3}}\right|
\end{aligned}
$$

where (as before) $\mathbf{t}=\left\{t_{1}, t_{2}, t_{3}\right\}$ and $\omega_{i t_{i}}$ represents the Gaussian quadrature weighting factor associated with the $i^{\text {th }}$ degree of freedom and the $t_{i}^{\text {th }}$ one-dimensional DVR 
point within that degree of freedom. In the case of a DVR derived from sets of orthogonal polynomials, these weighting factors are the corresponding Gaussian quadrature weights $[35,29,34,28]$. In the case of the equally-spaced sinc-function DVR $[41,44,94]$, where

$$
\operatorname{sinc}(x)=\frac{\sin x}{x}
$$

the weights logically correspond to $\Delta x$, the distance between DVR points.

Unfortunately, there is no easy prescription for determining the weighting factors to be used in the presently employed potential-optimized DVR. The finite basis representation most directly associated with this radial DVR (the eigenfunctions of the hydrogen 2 static potential) are not associated with any Gaussian quadrature scheme. Therefore the matrix of weights $\Delta$ (in the notation of Light [29, p. 1403]) which is associated with its quadrature is not diagonal in the point index and unique weights are not able to be assigned to each DVR point. Without such an assignment the simple evaluation of (3.139) cannot be used to provide values for $\mathbf{M}_{0}$. And since (in general) the number of composite DVR points is quite large, the number of elements in $\mathbf{M}_{0}$ prohibits a computationally intensive method of evaluating these multi-dimensional integrals.

The key to evaluating $\mathbf{M}_{0}$ lies in the realization that the DVR representation of the Hamiltonian given by (3.83) through (3.87) is inherently sparse. This means that the multiplicative application of the Hamiltonian operator on a state vector is a computationally inexpensive operation. (This, of course, is one of the primary reasons for working in a DVR representation.) Assuming that the composite potential-optimized DVR is sufficiently complete, unity can be inserted into (3.11) to get

$$
\left(\mathbf{M}_{0}\right)_{\mathbf{t n}}=\sum_{\mathbf{t}^{\prime}}\left\langle u_{\mathbf{t}}|\hat{H}-\hat{E}| u_{\mathbf{t}^{\prime}}\right\rangle\left\langle u_{\mathbf{t}^{\prime}} \mid \Psi_{0 \mathbf{n}}\right\rangle
$$

Now if the factor $\left\langle u_{t}|\hat{H}-\hat{E}| u_{t^{\prime}}\right\rangle$ is assumed to be available in the form of a subroutine which takes advantage of the inherent sparsity of the Hamiltonian to enable efficient multiplicative operation of the Hamiltonian onto a given column vector of the same dimensionality, the calculation of $\mathbf{M}_{0}$ is then reduced to calculating and storing the values of the projections $\left\langle u_{\mathbf{t}^{\prime}} \mid \Psi_{0 n}\right\rangle$. It is not apparent at first that any real progress 
has been made, except when one realizes that the analytical form for $\Psi_{0 n}$ is given by (3.96) as

$$
\Psi_{0 \mathbf{n}}\left(R_{1}, R_{2}, \gamma\right)=\sum_{i} F_{\mathbf{n} i}\left(R_{1}\right) \phi_{i}\left(R_{2}, \gamma\right)
$$

Therefore $\Psi_{0 n}$ is a simple sum of products of one-dimensional functions. (Of course, this is not in general true for $(\hat{H}-\hat{E}) \Psi_{0 n}$ because of the eigenstate mixing effect of the inter-electron potential.) The three-dimensional projection given by $\left\langle u_{\mathbf{t}^{\prime}} \mid \Psi_{0 \mathbf{n}}\right\rangle$ is easily calculated in terms of its component factors $\left\langle\varphi_{t_{1}} \mid F_{\mathbf{n} i}\right\rangle,\left\langle\varphi_{t_{2}} \mid \phi_{i}^{R}\right\rangle$, and $\left\langle\xi_{t_{3}} \mid \phi_{i}^{A}\right\rangle$ where $\varphi$ and $\xi$ are the radial and angular DVR functions defined by (3.54) and (3.61) respectively, $\phi_{i}^{R}$ and $\phi_{i}^{A}$ are the radial and angular portions of the $i^{\text {th }}$ open channel respectively. The three-dimensional projection is then given as

$$
\left\langle u_{\mathbf{t}} \mid \Psi_{0 \mathbf{n}}\right\rangle=\sum_{i}\left\langle\varphi_{t_{1}} \mid F_{\mathbf{n}_{i}}\right\rangle\left\langle\varphi_{t_{2}} \mid \phi_{i}^{R}\right\rangle\left\langle\xi_{t_{3}} \mid \phi_{i}^{A}\right\rangle
$$

Evaluation of the one-dimensional radial projections is accomplished by calculating the projections of $F_{\mathbf{n} i}$ and $\phi_{i}^{R}$ onto the original Sturmian basis functions described by (3.44) using Gauss-Laguerre quadrature, and using the transformation matrix $\mathbf{Z}_{12}$ defined by (3.53) to calculate the projections onto the one-dimensional DVR functions. Evaluating the one-dimensional angular projections is even simpler given that the angular portion of the hydrogen bound states corresponds exactly to the finite basis representation used to generate the Gauss-Legendre DVR. Projections are calculated by using (3.60). It is then a simple matter to calculate the quantity $\left\langle u_{\mathbf{t}^{\prime}} \mid \Psi_{0 \mathbf{n}}\right\rangle$ and store it in a column vector.

This procedure for calculating the bound-free elements is very much in the spirit of utilizing Lanczos methods for performing matrix inversions in the solution of simultaneous equations [32]. The generation of a Krylov space in such a procedure involves many multiplications of an initial column vector by the Hamiltonian matrix. In this case, the initial column vectors are simply the representations of the free functions $\Psi_{0 n}$ within the space spanned by the DVR. A single Hamiltonian multiplication (along with the total energy term) is sufficient to give $(\hat{H}-\hat{E}) \Psi_{0 n}$. 


\subsubsection{The Extended Radial DVR}

A cursory glance at (3.5) and (3.6)

$$
\begin{aligned}
& \mathbf{B}=\mathbf{M}_{00}-\mathbf{M}_{0}^{T} \cdot \mathbf{M}^{-1} \cdot \mathbf{M}_{0} \\
& \mathbf{C}=\mathbf{M}_{10}-\mathbf{M}_{0}^{* T} \cdot \mathbf{M}^{-1} \cdot \mathbf{M}_{0}
\end{aligned}
$$

together with the definitions of $\mathbf{M}_{00}, \mathbf{M}_{10}, \mathbf{M}_{\mathbf{0}}$, and $\mathbf{M}$ given in (3.7), (3.8), (3.10), and (3.11)

$$
\begin{aligned}
\left(\mathbf{M}_{00}\right)_{\mathbf{n n}^{\prime}} & =\left\langle\Psi_{0 \mathbf{n}}|\hat{H}-\hat{E}| \Psi_{0 \mathbf{n}^{\prime}}\right\rangle \\
\left(\mathbf{M}_{10}\right)_{\mathbf{n n}^{\prime}} & =\left\langle\Psi_{1 \mathbf{n}}|\hat{H}-\hat{E}| \Psi_{0 \mathbf{n}^{\prime}}\right\rangle \\
\mathbf{M}_{\mathbf{t} \mathbf{t}^{\prime}} & =\left\langle u_{\mathbf{t}}|\hat{H}-\hat{E}| u_{\mathbf{t}^{\prime}}\right\rangle \\
\left(\mathbf{M}_{0}\right)_{\mathbf{t n}} & =\left\langle u_{\mathbf{t}}|\hat{H}-\hat{E}| \Psi_{0 \mathbf{n}}\right\rangle
\end{aligned}
$$

reveal that if the DVR basis set used to represent $M$ is exactly the same as that used to generate $\mathbf{M}_{0}$ by the multiplicative method described in the previous section, the values of $\mathbf{B}$ and $\mathbf{C}$ generated will necessarily vanish, assuming that $L^{2}$ DVR basis is complete. Direct substitution into $(3.5)$ gives

$$
\begin{aligned}
(\mathbf{B})_{\mathbf{n} \mathbf{n}^{\prime}}= & \left(\mathbf{M}_{\mathbf{0 0}}\right)_{\mathbf{n} \mathbf{n}^{\prime}}-\left(\mathbf{M}_{0}^{T} \cdot \mathbf{M}^{-1} \cdot \mathbf{M}_{0}\right)_{\mathbf{n} \mathbf{n}^{\prime}} \\
= & \left\langle\Psi_{0 \mathbf{n}}|\hat{H}-\hat{E}| \Psi_{0 \mathbf{n}^{\prime}}\right\rangle- \\
& \sum_{\mathbf{t} \mathbf{t}^{\prime}}\left(\Psi_{0 \mathbf{n}}|\hat{H}-\hat{E}| u_{\mathbf{t}}\right\rangle\left\langle u_{\mathbf{t}}\left|(\hat{H}-\hat{E})^{-1}\right| u_{\mathbf{t}^{\prime}}\right\rangle\left\langle u_{\mathbf{t}^{\prime}}|\hat{H}-\hat{E}| \Psi_{0 \mathbf{n}^{\prime}}\right\rangle \\
= & \left\langle\Psi_{0 \mathbf{n}}|\hat{H}-\hat{E}| \Psi_{0 \mathbf{n}^{\prime}}\right\rangle- \\
& \left\langle\Psi_{0 \mathbf{n}}\left|(\hat{H}-\hat{E})(\hat{H}-\hat{E})^{-1}(\hat{H}-\hat{E})\right| \Psi_{0 \mathbf{n}^{\prime}}\right\rangle \\
= & 0 .
\end{aligned}
$$

The matrix $C$ vanishes in a similar way. It is fairly easy to see what the difficulty is. The quantity $(\hat{H}-\hat{E}) \Psi_{0 n}$ vanishes for $R_{1} \rightarrow \infty$ (since the reciprocal $R_{1}$ expansion for $\Psi_{0 n}$ solves the coupled-channel equations in the asymptotic region), but this is obviously not true for $\Psi_{0 n}$ itself. Therefore any attempt to represent $\Psi_{0 n}$ in an $L^{2}$ representation is certain to fail since the proper boundary condition for the free function cannot be achieved. This difficulty appears frequently in scattering calculations using basis sets and is discussed elsewhere by Jang and Light [95]. 
But in actuality, for the purposes of calculating the bound-free matrix element $M_{0}$ it isn't necessary that $\Psi_{0 n}$ be represented correctly throughout the entire scattering coordinate. It is merely necessary that $\Psi_{0 n}$ be accurately represented throughout the interaction region being spanned by the $L^{2}$ DVR grid basis. Now, the DVR grid basis is obviously composed of very localized functions. And the functions which have their particle density the furthest from the origin are not likely to adequately represent either $\Psi_{0 n}$ or its second derivative (arising from the kinetic energy operator) without additional contributions from DVR basis functions in neighborhoods around themselves. But this is not possible since it has been posited that these DVR basis functions were the ones with density furthest from the origin. The solution to this dilemma is presented here.

1. Create a different (more radially-extensive) DVR basis set to represent the free function $\Psi_{0 n}$ as well as the Hamiltonian matrix $\mathbf{H}$.

2. Multiply this expanded Hamiltonian matrix by a column matrix representing the free function $\Psi_{0 n}$ in the same expanded representation.

3. Project the resulting column matrix into the original DVR basis set.

Of course, there is nothing which says that the expanded $L^{2}$ basis set must be a DVR basis set. It is only necessary that it span the required space. But there are many benefits to using another DVR basis set which includes in it the original restricted DVR basis set. The required multiplication of the Hamiltonian $H$ by the column matrix representing $\Psi_{0 \mathbf{n}}$ is much less time-consuming if the Hamiltonian is sparse, and storage requirements are greatly minimized. In addition, the process of projecting the column matrix representing $(\hat{H}-\hat{E}) \Psi_{0 n}$ in the expanded space into the more restricted space involves merely setting certain elements in the original column vector to zero (or even omitting them). This is much easier than using stored overlap matrices to calculate the projection into a dissimilar basis set. Also, the bookkeeping routines used for generating parameters needed to perform the sparse Hamiltonian multiplication are very computationally inexpensive and it is very easy to reuse them multiple times for different sets of DVR functions. Finally, merely extending the 
DVR basis further in the scattering coordinate preserves the same density of DVR points in this degree of freedom. Since the same range of kinetic energies needs to be represented by both the restricted and extended DVR basis sets, it is logical that these densities should be the same.

To summarize in equation form, the new forms for the $\mathbf{B}$ and $\mathbf{C}$ matrices are given as

$$
\begin{aligned}
& \mathbf{B}=\mathbf{M}_{00}-\left(\tilde{\mathbf{v}}^{T} \cdot \tilde{\mathbf{M}} \cdot \hat{\mathbf{P}}^{T}\right) \cdot \mathbf{M}^{-1} \cdot(\hat{\mathbf{P}} \cdot \tilde{\mathbf{M}} \cdot \tilde{\mathbf{v}}), \quad \text { and } \\
& \mathbf{C}=\mathbf{M}_{10}-\left(\tilde{\mathbf{v}}^{* T} \cdot \tilde{\mathbf{M}} \cdot \hat{\mathbf{P}}^{T}\right) \cdot \mathbf{M}^{-1} \cdot(\hat{\mathbf{P}} \cdot \tilde{\mathbf{M}} \cdot \tilde{\mathbf{v}})
\end{aligned}
$$

where $\hat{\mathbf{P}}$ is a left-handed projection operator which projects from the extended DVR space into the restricted DVR space. The tilde indicates representation in the extended DVR basis set such that $\tilde{\mathbf{M}}$ is $\mathbf{H}-\mathbf{E}$ in the extended DVR space, and $\tilde{\mathbf{v}}$ is the column matrix for the free function $\Psi_{\text {on }}$ whose elements are defined as

$$
\begin{aligned}
(\tilde{\mathbf{v}})_{\tilde{t}_{n}} & =\left\langle u_{\tilde{\mathbf{t}}} \mid \Psi_{0 \mathbf{n}}\right\rangle \\
& =\sum_{i}\left\langle\varphi_{\tilde{t}_{1}} \mid F_{\mathbf{n} i}\right\rangle\left\langle\varphi_{\tilde{t}_{2}} \mid \phi_{i}^{R}\right\rangle\left\langle\xi_{\tilde{t}_{3}} \mid \phi_{i}^{A}\right\rangle
\end{aligned}
$$

(It is again temporarily assumed that the $L^{2}$ DVR functions $u_{\mathbf{t}}$ are not symmetrized.)

\subsubsection{Symmetrization of the Free Functions}

By the same arguments presented in the section dealing with the symmetrization of the $L^{2}$ Hamiltonian matrix elements, incorporation of proper spatial symmetry into the free functions offers a complete separation of the singlet $(S=0)$ and triplet $(S=1)$ computations. Symmetrization of the free functions $\Psi_{\text {on }}$ block diagonalizes the $\mathbf{M}_{\mathbf{0 0}}$ and $\mathbf{M}_{10}$ matrices defined by (3.7) and (3.8), thereby block diagonalizing the matrices $\mathbf{B}$ and $\mathbf{C}$ defined by (3.5) and (3.6). This in turn block diagonalizes the $\mathbf{S}$ matrix of (3.4). After incorporating a normalization factor which preserves the particle flux associated with the free functions, symmetrization produces the correct form for the free function

$$
\Psi_{0 \mathbf{n}}\left(R_{1}, R_{2}, \gamma\right)=\sum_{i} \frac{1}{\sqrt{2}}\left[F_{\mathbf{n} i}\left(R_{1}\right) \phi_{i}\left(R_{2}, \gamma\right) \pm F_{\mathbf{n} i}\left(R_{2}\right) \phi_{i}\left(R_{1}, \gamma\right)\right]
$$


Incorporating the properly derived symmetrization of the $L^{2}$ DVR functions given in (3.71) and (3.72) gives the projection formulae

$$
(\tilde{\mathbf{v}})_{\tilde{t}_{n}}=\sum_{i}\left[\left\langle\varphi_{\tilde{t}_{1}} \mid F_{\mathbf{n} i}\right\rangle\left\langle\varphi_{\tilde{t}_{2}} \mid \phi_{i}^{R}\right\rangle\left\langle\xi_{\tilde{t}_{3}} \mid \phi_{i}^{A}\right\rangle \pm\left\langle\varphi_{\tilde{t}_{2}} \mid F_{\mathbf{n} i}\right\rangle\left\langle\varphi_{\tilde{t}_{1}} \mid \phi_{i}^{R}\right\rangle\left\langle\xi_{\tilde{t}_{3}} \mid \phi_{i}^{A}\right\rangle\right]
$$

for the case where $\tilde{t}_{1}>\tilde{t}_{2}$, and

$$
(\tilde{\mathbf{v}})_{\tilde{\mathbf{t}}_{\mathfrak{n}}}=\sum_{i} \sqrt{2}\left\langle\varphi_{\tilde{t}_{1}} \mid F_{\mathbf{n} i}\right\rangle\left\langle\varphi_{\tilde{t}_{2}} \mid \phi_{i}^{R}\right\rangle\left\langle\xi_{\tilde{t}_{3}} \mid \phi_{i}^{A}\right\rangle
$$

for the singlet scattering case where $\tilde{t}_{1}=\tilde{t}_{2}$. (Once again, the tilde indicates representation in the extended DVR space.) For the triplet scattering case where $\tilde{t}_{1}=\tilde{t}_{2}$, this matrix element vanishes. As before, the " + " option indicates singlet scattering and the "-" option indicates triplet scattering.

\subsection{Calculation of the Free-Free Matrix Elements}

Since the form of the cutoff function presented in (3.135) is of a somewhat complicated form, an analytical solution to the free-free matrix elements is not practically possible. And while the extended radial DVR method which was used in the calculation of the bound-free matrix $M_{0}$ may also be used in the calculation of the free-free matrices $M_{00}$ and $M_{10}$ defined by (3.7) and (3.8), the number of open channels present in this calculation is only three and so the dimensionality of the $\mathbf{M}_{00}$ and $\mathbf{M}_{10}$ matrices is quite small. Instead, these matrices were calculated by the use of automated numerical integration routines provided by NAG (Numerical Algorithms Group). The calculation of the free-free matrix elements begins with (3.154)

$$
\Psi_{0 \mathbf{n}}\left(R_{1}, R_{2}, \gamma\right)=\sum_{i} \frac{1}{\sqrt{2}}\left[F_{\mathbf{n} i}\left(R_{1}\right) \phi_{i}\left(R_{2}, \gamma\right) \pm F_{\mathbf{n} i}\left(R_{2}\right) \phi_{i}\left(R_{1}, \gamma\right)\right] .
$$

Since the form of the Hamiltonian given in (3.2) is invariant to exchange of $R_{1}$ and $\mathbf{R}_{2}$, substitution into (3.7) gives

$$
\begin{aligned}
\left(\mathbf{M}_{00}\right)_{\mathbf{n n}^{\prime}} & =\left\langle\Psi_{0 \mathbf{n}}|\hat{H}-\hat{E}| \Psi_{0 \mathbf{n}^{\prime}}\right\rangle \\
& =\sum_{i i^{\prime}}\left[\left\langle F_{\mathbf{n}_{i}} \phi_{i}|\hat{H}-\hat{E}| F_{\mathbf{n}^{\prime} i^{\prime}} \phi_{i^{\prime}}\right\rangle \pm\left\langle F_{\mathbf{n} i} \phi_{i}|\hat{H}-\hat{E}| \phi_{i^{\prime}} F_{\mathbf{n}^{\prime} i^{\prime}}\right\rangle\right]
\end{aligned}
$$


in which the first term in the summand is the direct term and the second term is the exchange term. As before, the order of the factors within the bra and ket determine whether the factor is a function of $R_{1}$ or $R_{2}$. It would be computationally advantageous to be able to assume that the exchange term vanishes, since this would cut in half the computational effort required for the calculation of each free-free matrix element.

Various methods have been proposed for the elimination of the free-free exchange terms. Rescigno and Schneider [96] perform an explicit Gramm-Schmidt orthogonalization of the free functions $\Psi_{0 n}$ to the composite $L^{2}$ basis set. Now, the action of the Hamiltonian $\hat{H}$ upon the free functions $\Psi_{0 \mathrm{n}}$ is likely to create components outside of this $L^{2}$ space. These are explicitly removed with the projection operator

$$
\hat{P}=\sum_{\mathbf{t}}\left|\Lambda_{\mathbf{t}}\right\rangle\left\langle\Lambda_{\mathbf{t}}\right|
$$

where $\Lambda_{t}$ is a composite function in the $L^{2}$ space. This ensures that the exchange matrix elements vanish.

Here, a more physically intuitive approach is taken. The form chosen for the cutoff function given in (3.135) is quite general. Because the hydrogen eigenfunctions are of limited spatial extent, the parameters composing the cutoff function can be chosen such that the cutoff function vanishes for the vast majority of the region of the scattering coordinate in which the hydrogen eigenfunctions have finite density. Because each of the operators in the Hamiltonian is either of a multiplicative form or a second derivative form, the action of $\hat{H}$ upon the free functions is unable to introduce particle density into the region where the cutoff function vanishes. It is therefore sufficient to introduce such parameters $A, B$, and $C$ into the cutoff function of (3.135) such that the function has negligible overlap with the hydrogen eigenfunctions. A glance at (3.158) indicates that this is sufficient to force the exchange term to vanish. This computationally simplifying approach is also utilized by Zhang and Miller [54].

The Hamiltonian is given in (3.2) as

$$
\hat{H}=-\frac{1}{2} \frac{\partial^{2}}{\partial R_{1}^{2}}-\frac{1}{2} \frac{\partial^{2}}{\partial R_{2}^{2}}+\left(\frac{1}{2 R_{1}^{2}}+\frac{1}{2 R_{2}^{2}}\right) \hat{L}^{2}-\frac{1}{R_{1}}-\frac{1}{R_{2}}+\frac{1}{\left|\mathbf{R}_{1}-\mathbf{R}_{2}\right|} .
$$


For each term in the Hamiltonian other than that describing the inter-electron interaction, integrating out the $R_{2}$ and $\gamma$ degrees of freedom is a relatively simple matter to perform analytically since the analytical form of the hydrogen eigenfunctions is very simple. (Here, it is arbitrarily assumed that $\phi_{i}$ is a function of $R_{2}$ and not $R_{1}$.) For these terms this enables one to obtain a simple form for the integrand in the one degree of freedom remaining $-R_{1}$. For the term describing the inter-electron degree of freedom, the summand of the integrand for the $R_{1}$ integration is given as

$$
\left\langle F_{\mathbf{n} i} \phi_{i}\left|\frac{1}{\left|\mathbf{R}_{1}-\mathbf{R}_{2}\right|}\right| F_{\mathbf{n}^{\prime} i^{\prime}} \phi_{i^{\prime}}\right\rangle_{R_{2} \gamma}=F_{\mathbf{n} i}\left(R_{1}\right) F_{\mathbf{n}^{\prime} i^{\prime}}\left(R_{1}\right)\left\langle\phi_{i}\left|\frac{1}{\left|\mathbf{R}_{1}-\mathbf{R}_{2}\right|}\right| \phi_{i^{\prime}}\right\rangle_{R_{2} \gamma}
$$

where (as described previously) the subscript $R_{2} \gamma$ indicates the variables over which integration is to be performed. The standard multipole expansion then gives

$$
\left\langle\phi_{i}\left|\frac{1}{\left|\mathbf{R}_{1}-\mathbf{R}_{2}\right|}\right| \phi_{i^{\prime}}\right\rangle_{R_{2} \gamma}=\sum_{l=0}^{l_{\max }} \frac{\sqrt{\left(2 l_{i}+1\right)\left(2 l_{i^{\prime}}+1\right)}}{2 l+1}\left[C\left(l_{i} l_{i^{\prime}} ; 000\right)\right]^{2}\left\langle\phi_{i}^{r}\left|\frac{R_{<}^{l}}{R_{>}^{l+1}}\right| \phi_{i^{\prime}}^{r}\right\rangle_{R_{2}}
$$

where the angle $\gamma$ has been integrated out and notation similar to that in (3.99) is used. The integration over $R_{2}$ present in the last term on the right-hand side is calculated numerically using an automated NAG routine. This is performed for each applicable value of $R_{1}$ to obtain and integrand dependent only upon $R_{1}$.

Using this routine for the calculation of the integrand as a function of $R_{1}$, the free-free matrix elements of $M_{00}$ and $M_{10}$ are determined using another NAG automated integration routine. It will be noticed that no discontinuous or cusp behavior is to be found in the $R_{1}$ integrand since the spline fit used in calculating the free function $F_{\mathbf{n} i}$ matched $0^{\text {th }}$ through $3^{\text {rd }}$ order derivatives at the demarcation point between the reciprocal $R_{1}$ expansion and the two-frequency sine/cosine expansion used in (3.130) for the interaction region. This lack of bad behavior in the $R_{1}$ integrand provides wider freedom in deciding which automated integration routines can be used effectively, and there is no need to split the integration into separate regions. 


\section{Chapter 4}

\section{Results and Discussion}

\subsection{Setting Parameter Values}

From the previous chapter detailing the theoretical background used in developing a discrete variable representation (DVR) useful for treating electron-atom collisions, it can be seen that there are numerous parameters involved in such a calculation. Certainly, there are optimum values for each of these parameters which maximize the efficiency of the calculation. Some of the values of these parameters were determined based upon reasonable physical determinations. Other values were used as convergence parameters, whereby values of the particular parameter were varied until stable values for $\mathbf{S}$ matrices and cross sections were achieved. This section describes the procedures by which the parameters present in the scattering calculations were determined.

\subsubsection{Setting up the Radial DVR}

As was described within the previous theoretical chapter, the development of the radial DVR involves a series of two diagonalizations. The first diagonalization involves calculating eigenfunctions of the direct portion of the strengthened static hydrogen $2 s$ potential energy surface given by (3.29) as

$$
\tilde{V}_{2 s}(R)=-\alpha e^{-R}\left(\frac{1}{R}+\frac{3}{4}+\frac{1}{4} R+\frac{1}{8} R^{2}\right)
$$


where $\alpha$ is the strength parameter. A primitive basis set of Sturmian functions is used in this diagonalization. These Sturmian functions are defined in (3.44) as

$$
\phi_{n}(x)=\sqrt{\frac{s}{n(n+1)}} e^{-s x / 2} s x L_{n}(s x)
$$

where $s$ is a scale parameter. It is necessary to determine the total number of such Sturmian functions to include in the primitive basis set, as well as the value of the scale parameter $s$. An insufficient number of primitive basis functions will prevent the radial DVR from spanning a sufficient amount of the radial $L^{2}$ space. More specifically, high kinetic energy components of the scattering wave function near the origin will be badly represented. And since the zeroes of the Laguerre polynomials become widely spaced at large values of $R$, a large number of such functions (together with a large scale parameter) are needed to adequately represent the oscillations of the wavefunction at the edge of the interaction region. So it would seem that one should use as many primitive Sturmians as numerically feasible since the derivation of the radial DVR is only performed once for several scattering energies. In these calculations, the number of primitive Sturmians was set at 380. Attempts to use more than this resulted in numerical difficulties stemming from poor evaluations of $\tilde{V}_{2 s}$ matrix elements needed for the first diagonalization, most likely caused by poor evaluations of Gauss-Laguerre quadrature points and weights by the NAG routine employed.

The determination of the Sturmian scale parameter $s$ was found to be somewhat of a difficult point requiring some empirical examination and experimentation. As described previously, in the interest of computational efficiency it is worthwhile to confine the particle density of the radial DVR basis to only that region of the scattering coordinate which requires it (the interaction/exchange region). As such, it would seem that confining the particle density of the primitive Sturmian functions to the same region would be the natural thing to do. But it has actually been found that the best radial DVR results from allowing the primitive Sturmian functions to extend radially well beyond the maximum desired reach of the radial DVR functions.

With some reflection it is not difficult to understand why this is the case. For any particular set of primitive Sturmian functions $\left\{\phi_{n}\right\}$, only the function with 
the largest index $n$ will have appreciable particle density at the largest possible values of the scattering coordinate $R$. If the farthest reach of the highest indexed Sturmian function also corresponds to a region of the scattering coordinate which needs to be represented by the radial DVR, it is reasonable to assume that the DVR functions centered at high values of the scattering coordinate are not likely to be well-formed since they would need to be represented by the relatively small number of primitive Sturmians with particle density in this region.

It is particularly important that the highly localized radial DVR functions be well-formed. Since the kinetic energy matrix of the primitive Sturmians is known exactly by (3.48), the one-dimensional kinetic energy matrix for the DVR functions will also be exact. But, as in all discrete variable representations, the potential matrix elements of the composite DVR functions are approximated by the values of the potential energy surface at the composite DVR point. In order for this quadrature approximation to be accurate, it is important that the DVR functions be locally symmetrical. It has been found in this set of calculations that the only way to ensure that this requirement is met is to allow the primitive Sturmian basis to extend well beyond the anticipated interaction region.

Previously a prescription was suggested which determined the scale parameter $s$ by requiring that the final Gauss-Laguerre quadrature point used for calculating $\tilde{V}_{2 s}$ matrix elements of the primitive Sturmian functions be located at a particular value of the scattering coordinate. This prescription is followed in these calculations and the value of the scattering coordinate used for the final quadrature point has been set at $R=61 \mathrm{au}$, well beyond the end of the interaction region for all of the scattering energies studied in these calculations.

Naturally, the use of the extended radial DVR (which was previously described for the projection calculation of the bound-free matrix elements of $M_{0}$ ) also requires that the primitive Sturmian basis provide particle density beyond the furthest reach of the extended radial DVR. But it is found that the extended radial DVR need not provide much more than one or two extra radial DVR functions in order to generate an accurate approximation to $\mathrm{M}_{0}$, and the value of $R=61$ au previously given has been found to be quite adequate. 
Having determined the correct primitive Sturmian basis set, it is then necessary to determine the optimum multiplicative strength parameter $\alpha$ associated with the strengthened static 2 s potential given by (3.29)

$$
\tilde{V}_{2 s}(R)=-\alpha e^{-R}\left(\frac{1}{R}+\frac{3}{4}+\frac{1}{4} R+\frac{1}{8} R^{2}\right) \text {. }
$$

As was mentioned previously, one wishes to avoid choosing a value for $\alpha$ which is too close to zero since this will generate a radial DVR which is composed of equally-spaced points which will not adequately sample the region of the potential energy surface close to the origin where the Coulomb interaction is strongest. Likewise, choosing a value for $\alpha$ which is too large will generate a radial DVR which has too sparse a distribution of points at the edge of the interaction region, such as in standard GaussLaguerre DVR. This might be adequate in a bound state calculation where the edge of the interaction region is dominated by decaying exponential wavefunctions. But it would certainly not be so in a scattering calculation at typical asymptotic kinetic energies.

A clue is suggested by the form of (3.12), which gives the differential equation satisfied by the Sturmian functions:

$$
\left(-\frac{1}{2} \frac{d^{2}}{d R^{2}}+\frac{l(l+1)}{2 R^{2}}-\frac{\alpha_{n l}}{R}-E\right) S_{n l}(R)=0 .
$$

In this equation, $\alpha_{n l}$ is taken to be proportional to $n$. In the same way, one can expect the proportionality constant $\alpha$ associated with the strengthened 2 s static potential $\tilde{V}_{2 s}$ to give better results when its value is roughly proportional to the number of radial DVR functions to be derived from the two successive diagonalizations used in generating the DVR. It would be instructive at this point to examine different distributions of radial DVR points derived from various values of the strength parameter $\alpha$. Figures 4.1 and 4.2 show such distributions for 50 radial DVR points.

It is apparent here that as the strength parameter $\alpha$ is increased, the density of DVR points near the origin increases greatly. (Notice that the equally-spaced DVR resulting from setting $\alpha=0$ places its first radial DVR point more than a full atomic unit from the origin.) It is also apparent that since the total number of radial DVR points within the given range is constant, increasing the density of DVR points near 


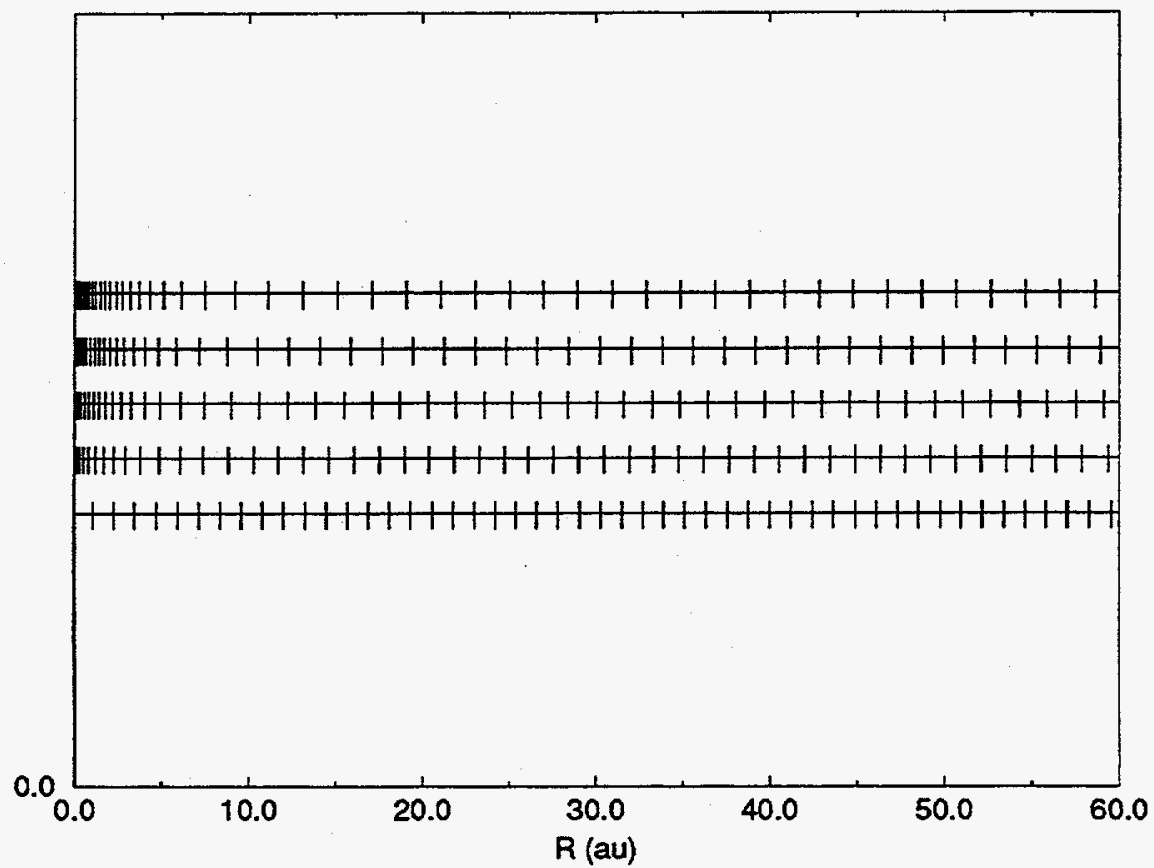

Figure 4.1: Radial DVR distributions for different values of the strength parameter $\alpha$. From bottom to top: $\alpha=0, \alpha=40, \alpha=80, \alpha=120, \alpha=160$.

the origin necessarily decreases the density in the asymptotic region. In this way, the strength parameter $\alpha$ can be thought of as a tuning parameter which can provide a compromise between equally-spaced DVR and a DVR with greater variations in point density (such as Gauss-Laguerre DVR). The strength parameter $\alpha$ will be thoroughly examined as a convergence parameter later.

It is useful at this point to actually examine the functional form of the localized DVR functions, both deep within the interaction region where the strengthened static $2 s$ potential $\tilde{V}_{2 s}$ has a strong Coulombic character

$$
\tilde{V}_{2 s} \sim-\frac{\alpha}{R},
$$

and also within the more electronically shielded environment outside of the particle density of the bound hydrogen electron where

$$
\tilde{V}_{2 s} \sim-\frac{\alpha}{8} R^{2} e^{-R} .
$$




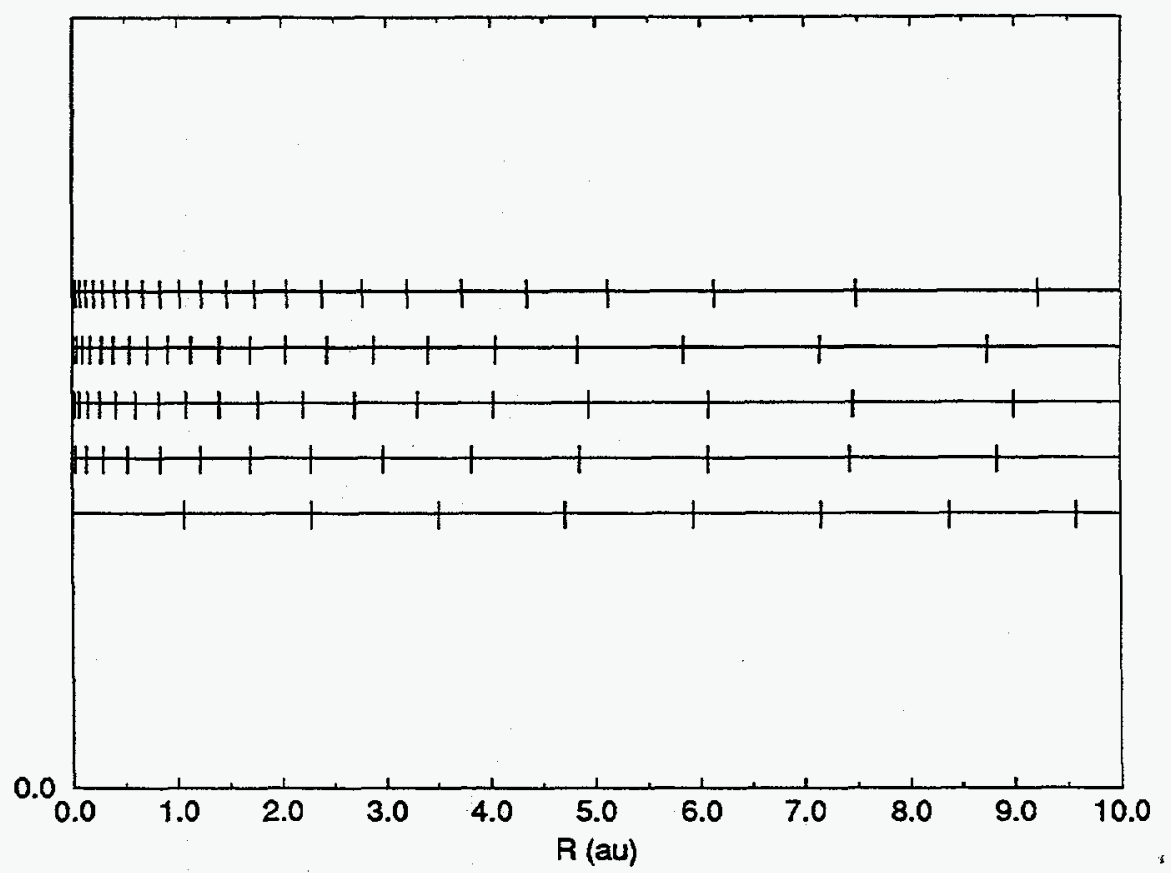

Figure 4.2: Radial DVR distributions within the interaction region for different values of the strength parameter $\alpha$. From bottom to top: $\alpha=0, \alpha=40, \alpha=80, \alpha=120$, $\alpha=160$.

Figure 4.3 shows that the radial DVR functions located in the shielded region of the scattering coordinate look very similar to the sinc-functions of (3.142) which form the basis for the DVR of Colbert and Miller [41]. In fact, the one-dimensional kinetic energy matrix elements formed between pairs of these calculated asymptotic DVR functions are almost identical to those resulting from this sinc function DVR. This is to be contrasted with Figure 4.4 which shows an expanded view of one of the calculated DVR functions located well within the interaction region of the scattering coordinate where the shielding of the charge of the proton is low. This function is similar to the sinc function in that its amplitude vanishes (or nearly so) at the values of the associated DVR points. But of course the density of the DVR points is not constant within this region.

Figure 4.5 shows the calculated DVR function associated with a point near the end of the region covered by the primitive Sturmian function basis set. It can be seen here that while the section of greatest particle density for this function appears 


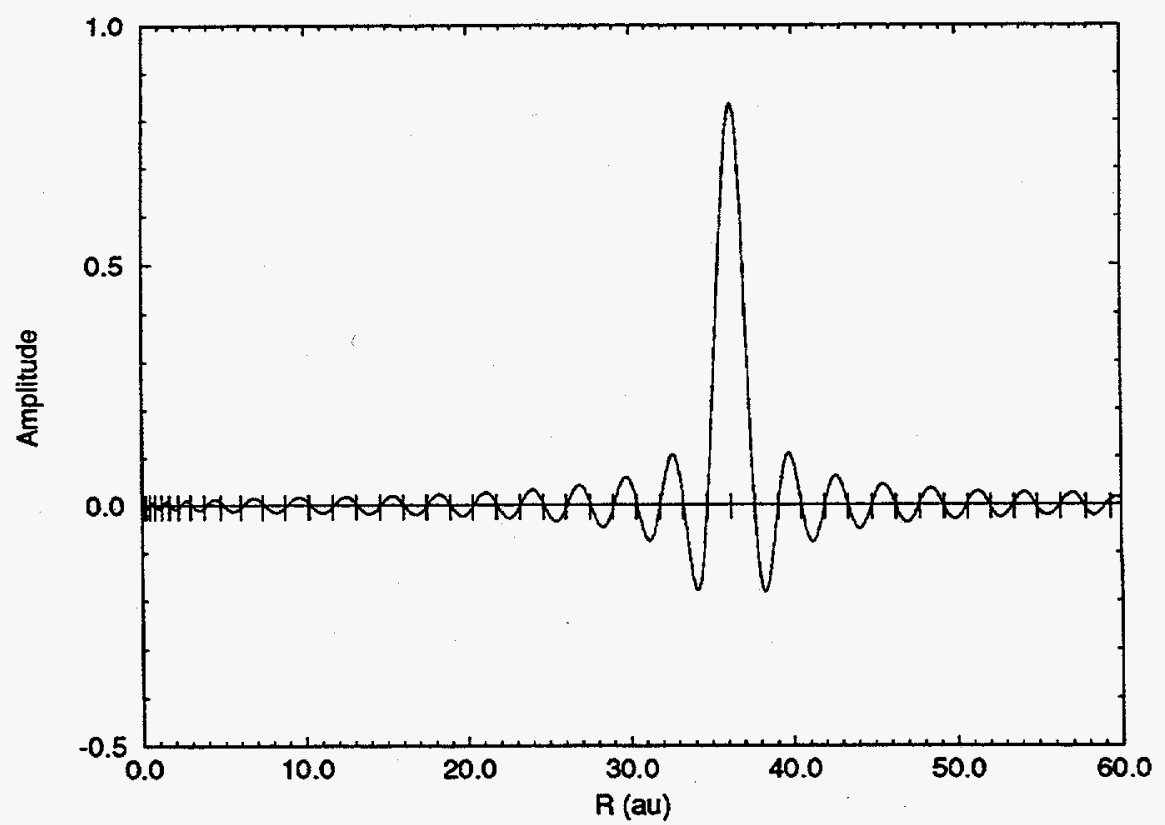

Figure 4.3: Radial DVR function toward the end of the interaction region. Small vertical lines indicate positions of radial DVR points computed with strength parameter $\alpha=40$.

to be relatively well-behaved and symmetrical, the remainder of the domain of the function is extremely irregular. This is the main reason for requiring that the primitive Sturmian basis span a physical space extending well beyond the anticipated domain of both the restricted radial DVR (used in the Hamiltonian matrix inversion) and the extended radial DVR (used in calculating the free-free matrix elements). The irregularities present in the function shown in Figure 4.5 ensure that this function would not offer an accurate quadrature approximation of the potential energy at any composite DVR point which depends upon this one-dimensional function.

\subsubsection{Setting up the Free Function Expansion}

In this section the development of the free function $\Psi_{0 n}$ is discussed. While there are a moderately large number of parameters associated with these functions, many of them can be set simply on the basis of physical constraints and need not be used as convergence parameters. By way of review, the free function is given in 


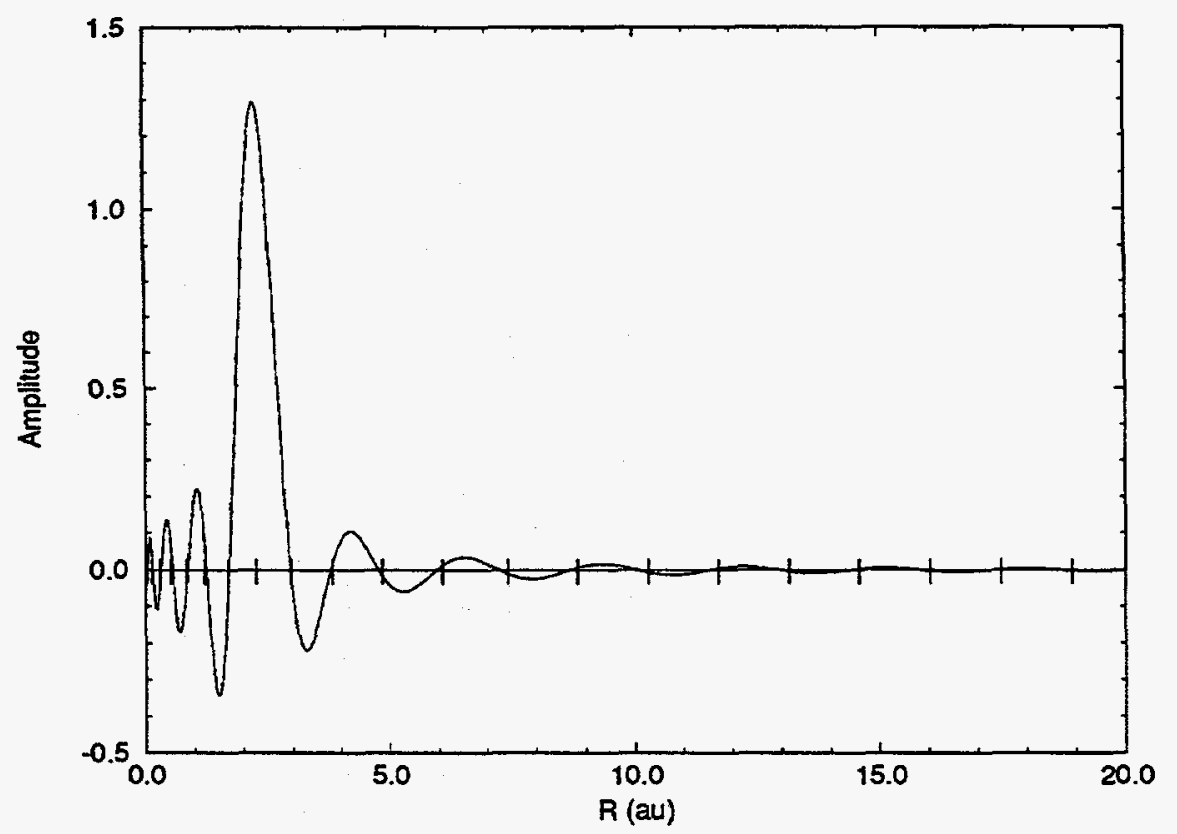

Figure 4.4: Radial DVR function within the interaction region. Small vertical lines indicate positions of radial DVR points computed with strength parameter $\alpha=40$.

(3.96) the form

$$
\Psi_{0 \mathbf{n}}\left(R_{1}, R_{2}, \gamma\right)=\sum_{i} F_{\mathbf{n} i}\left(R_{1}\right) \phi_{i}\left(R_{2}, \gamma\right)
$$

where $\mathbf{n}$ is the open channel index, $\phi_{i}$ is the $i^{\text {th }}$ hydrogen atom quantum state, and $F_{\mathbf{n} i}$ is the one-dimensional free function associated with this state. As described previously, the functions $F_{\mathbf{n} i}$ take the form of (3.103) multiplied by a cutoff function giving

$$
F_{\mathbf{n} i}(R)=f(R) \sum_{\kappa=1}^{c_{o}} \sum_{p=0}^{p_{\max }}\left[\alpha_{p}^{\mathbf{n} i \kappa} \sin \left(k_{\kappa} R\right)+\beta_{p}^{\mathbf{n} i \kappa} \cos \left(k_{\kappa} R\right)\right] R^{-p}
$$

when $R>R_{0}$ ( $R_{0}$ being a convenient demarcation point separating the exchange region from the asymptotic region which has been set at 20 au in these calculations) and $f(R)$ is the cutoff function defined by (3.135). Within the exchange region when $R<R_{0}, F_{\mathbf{n} i}$ takes the form of the spline fit in (3.130) multiplied by the same cutoff function, giving

$$
F_{\mathbf{n} i}(R)=f(R)\left[c_{\mathbf{n} i 1} \sin k_{a}(R)+c_{\mathbf{n} i 2} \cos k_{a}(R)+c_{\mathbf{n} i 3} \sin k_{b}(R)+c_{\mathbf{n} i 4} \cos k_{b}(R)\right] .
$$




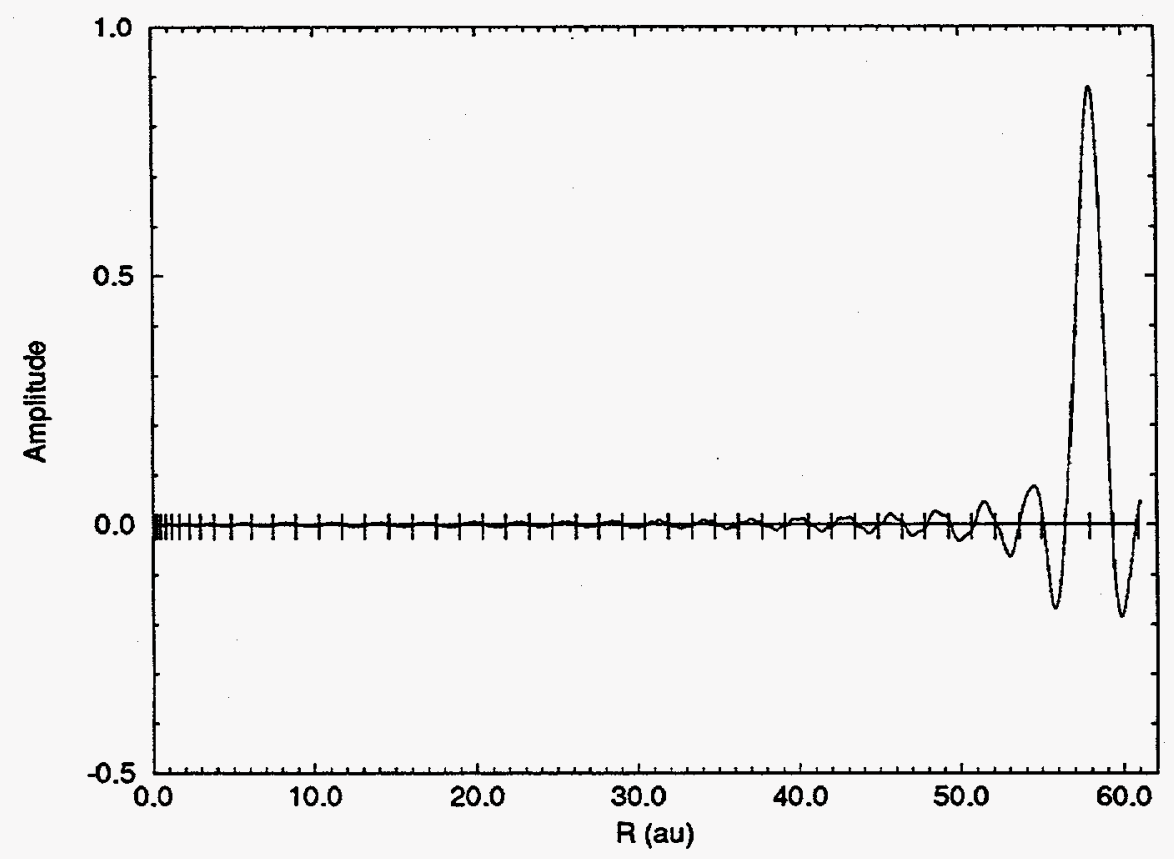

Figure 4.5: Radial DVR function toward the end of the extended DVR region. Small vertical lines indicate positions of radial DVR points computed with strength parameter $\alpha=40$.

The definition of the cutoff function given in (3.135) defines $f$ as

$$
f(R)=\frac{1}{\sqrt{\pi}} \int_{-\infty}^{u} e^{-t^{2}} d t
$$

where

$$
\begin{aligned}
& u=x+C x^{3}, \quad \text { and } \\
& x=A(R-B) .
\end{aligned}
$$

There are certain physical constraints which need to be satisfied for optimum results. The symmetrized form for the free-free matrix elements is given in (3.158) as

$$
\left(\mathbf{M}_{\mathbf{0 0}}\right)_{\mathbf{n} \mathbf{n}^{\prime}}=\sum_{i i^{\prime}}\left[\left\langle F_{\mathbf{n} i} \phi_{i}|\hat{H}-\hat{E}| F_{\mathbf{n}^{\prime} i^{\prime}} \phi_{i^{\prime}}\right\rangle \pm\left\langle F_{\mathbf{n} i} \phi_{i}|\hat{H}-\hat{E}| \phi_{i^{\prime}} F_{\mathbf{n}^{\prime} i^{\prime}}\right\rangle\right]
$$

In order to be able to assume that the exchange term in this equation vanishes it is necessary to choose a combination of parameters $A, B$, and $C$ such that the overlap of the individual components $F_{\mathbf{n} i}$ of the free function have no overlap with 
the radial portions of the set of hydrogen bound state wavefunctions $\left\{\phi_{i}\right\}$. It is also desirable that the range of the cutoff function in which the value switches from 0 to 1 be minimal, since a long switching distance increases the size of the region of the scattering coordinate which needs to be covered by the DVR function space. Unfortunately, if the switching distance is too short this requires a high density of DVR points in this region. Optimally, the switching distance is then roughly proportional to the shortest wavelength which needs to be represented by the DVR grid. In this way, the physical wavefunction remains the factor which determines the density of DVR points, rather than the artificial cutoff function $f$. Figure 4.6 shows the spatial extent of the cutoff function used in this study together with the radial extent of the three hydrogen bound states present.

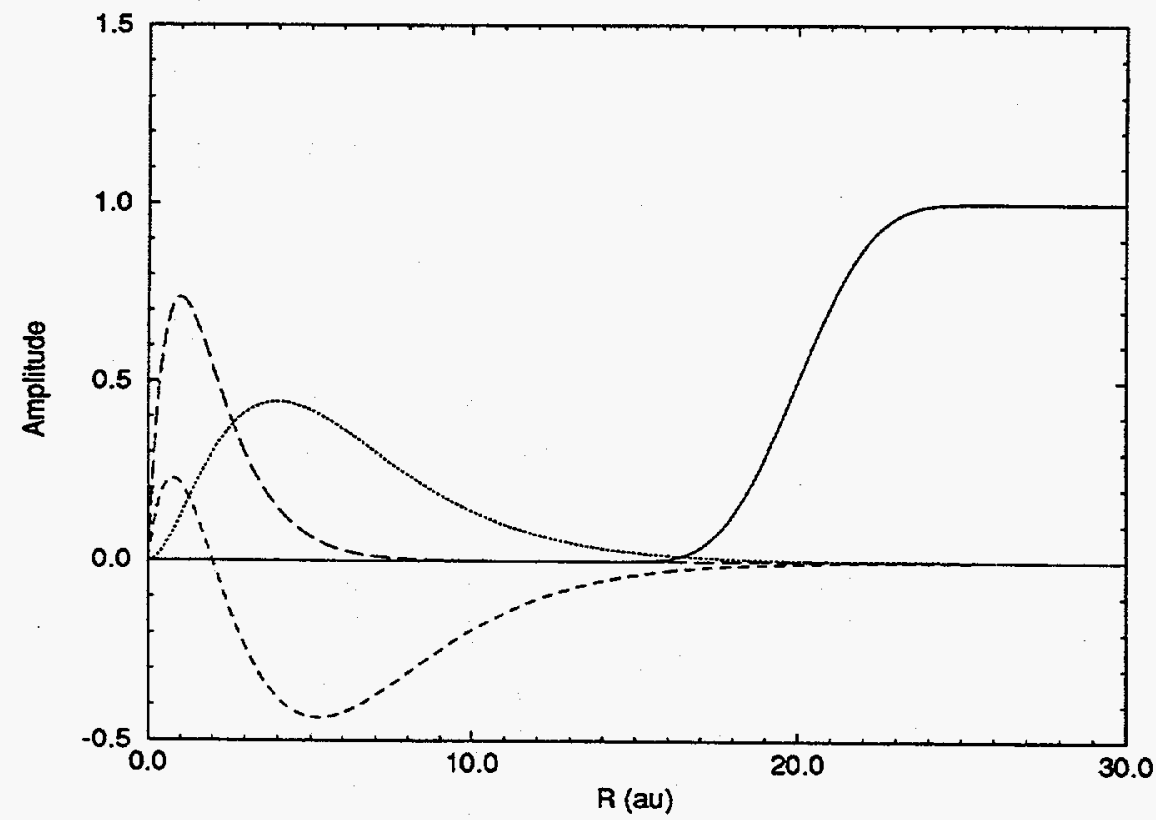

Figure 4.6: Cutoff function $f$ in comparison to $H$ bound states. Parameter values are $A=0.39, B=20.0$, and $C=0.05$. Solid line: $f(R)$; Long-dashed line: radial $\phi_{1 s}$ wavefunction; Dashed-line: radial $\phi_{2 s}$ wavefunction; Dotted-line: radial $\phi_{2 p}$ wavefunction.

Having set the parameters which determine the form of the cutoff function $f$, an examination of the representation of the free functions within the extended radial DVR is worthwhile since it offers an approximate idea of the density of radial DVR 
points required in the calculation. Since the density of radial DVR points required is directly related to the shortest wavelength present in the system, projections of free functions onto the radial DVR space are examined for the highest kinetic energies present, since these energies present the greatest challenge to the projection technique. For this study, this corresponds to the free function component associated with a $\phi_{1 s}$ state for an asymptotic $\phi_{1 s}$ state scattering at a total energy just below the $n=3$ threshold. The number of extended radial DVR points used equals the number of eigenvalues of the strengthened static $2 s$ potential $\left(\tilde{V}_{2 s}\right)$ used in the diagonalization of the position coordinate. Figures 4.7 through 4.10 show these projections for various numbers of extended radial DVR points.

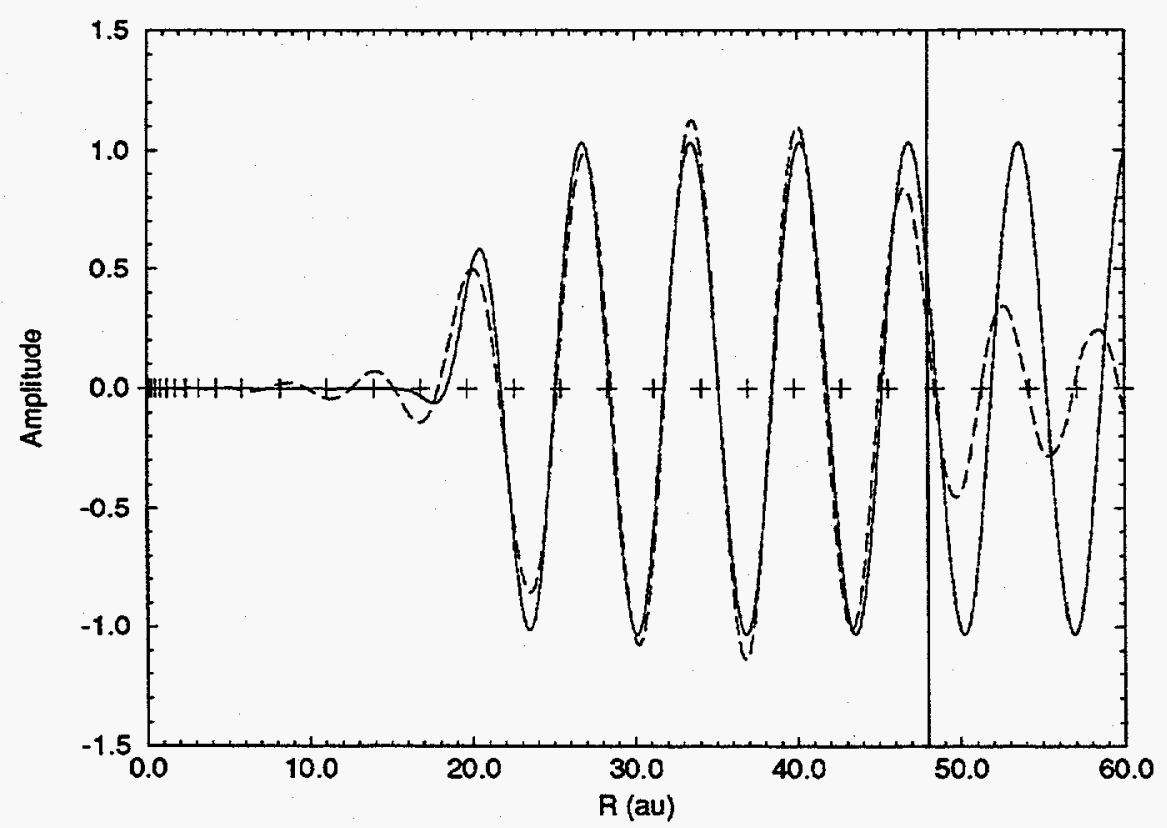

Figure 4.7: Projection of $F_{\mathbf{n} i}$ for $\mathbf{n}=1$ and $i=1$ for $E=0.88 \mathrm{Ryd}$ (30 $\hat{x}$ eigenstates). Solid line: analytical $F_{\mathbf{n} i}$; Dashed line: the projection approximation. Plus signs indicate radial DVR points and the long vertical line indicates the end of the extended radial DVR grid.

As can plainly be seen, the accuracy of the projection of the free function onto the extended DVR grid begins to become accurate when approximately four radial DVR points are made available per wavelength. The region where the analytical free function component vanishes (inside the interaction region) appears to be the 


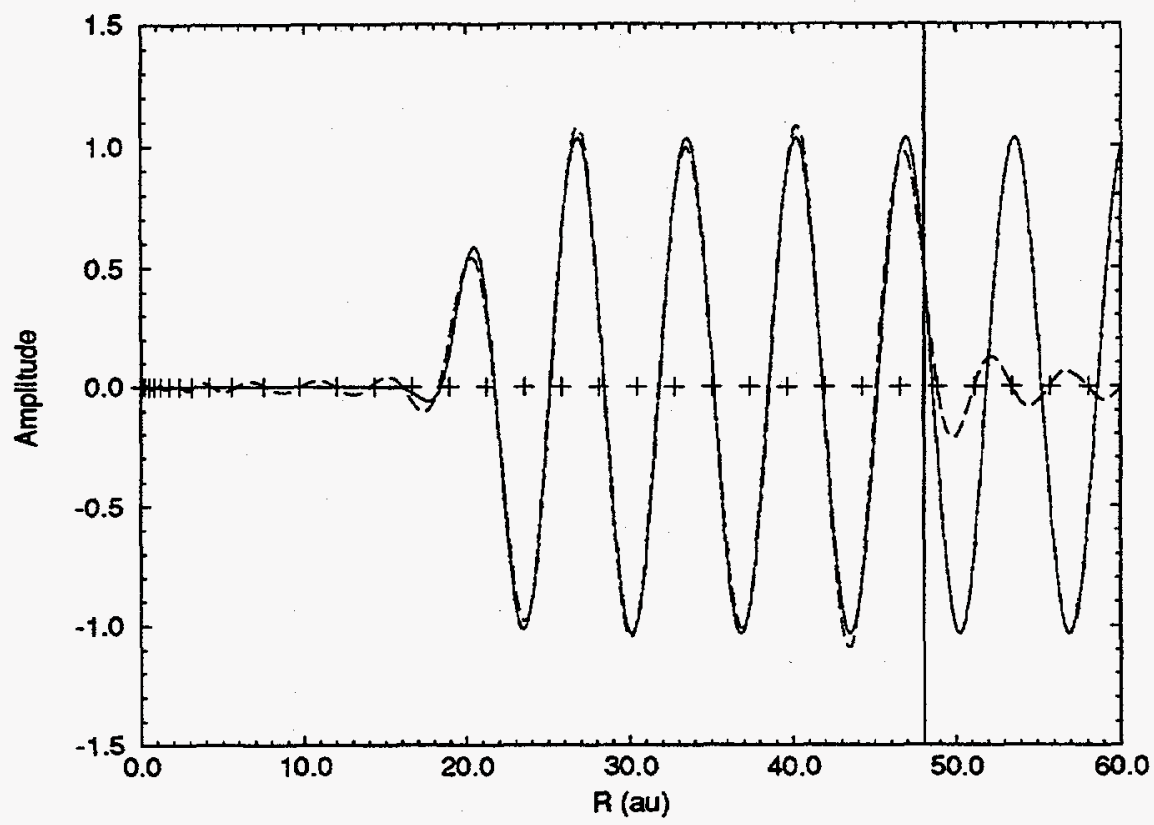

Figure 4.8: Projection of $F_{\mathbf{n} i}$ for $\mathbf{n}=1$ and $i=1$ for $E=0.88 \mathrm{Ryd}$ (35 $\hat{x}$ eigenstates). Solid line is analytical $F_{\mathbf{n} i}$; dashed line is the projection approximation. Plus signs indicate radial DVR points and the long vertical line indicates the end of the extended radial DVR grid.

most difficult region to emulate with the projection technique.

\subsection{Singlet and Triplet Scattering Calculations}

The calculations presented in this section were performed in double precision on a Sun SPARCstation 10. For reasons described in the next chapter of this thesis, the inversion of the $M$ matrix described in (3.10) was performed using a full-storage LU decomposition (with partial pivoting) followed by Gaussian elimination. Solution vectors were iteratively refined to achieve machine accuracy. Calculation of the $\mathbf{S}$ matrix for a given energy required approximately 25 minutes of CPU time and $120 \mathrm{MB}$ of core memory. The singlet calculations were performed using a matrix composed of 2380 composite DVR points; the triplet calculations were performed with 2520 composite DVR points. 


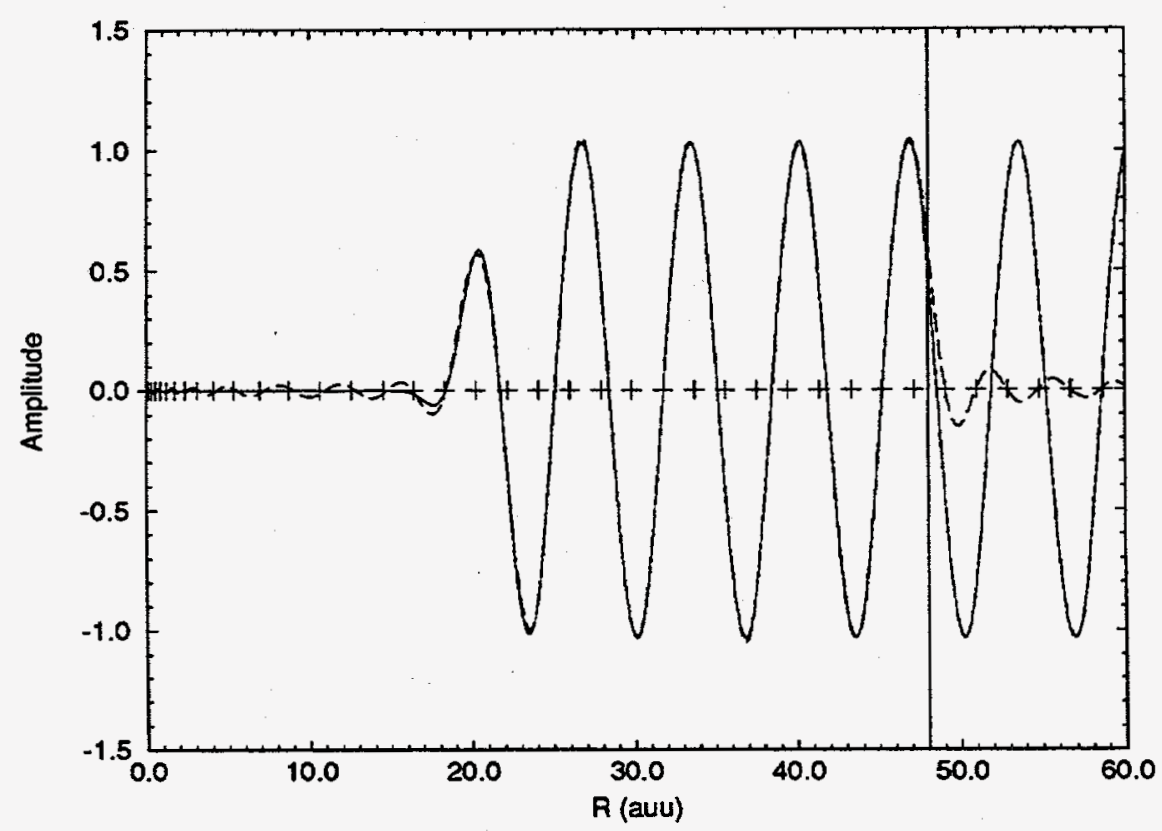

Figure 4.9: Projection of $F_{\mathbf{n} i}$ for $\mathbf{n}=1$ and $i=1$ for $E=0.88$ Ryd ( $40 \hat{x}$ eigenstates). Solid line is analytical $F_{n}$; dashed line is the projection approximation. Plus signs indicate radial DVR points and the long vertical line indicates the end of the extended radial DVR grid.

\subsubsection{Convergence of Singlet Transition Probabilities}

This section deals with the convergence of the calculation of transition probabilities for the singlet $(S=0)$ symmetry. Many of the convergence parameters determined here are used again in a later section without change for the calculation of transition probabilities for the triplet $(S=1)$ symmetry. To facilitate the reporting of these results, the values of parameters in the final results are presented with the understanding that the intermediate convergence tests (which examine only one parameter at a time) differ from the final parameter values only in the value of the parameter being studied in that particular convergence test.

As was mentioned previously, not all the parameters present in these calculations were used as convergence parameters. In particular, parameters associated with the formulation of the free functions (including those determining the cutoff function) were fixed at the previously reported values since these were seen to be physically reasonable and it was judged (correctly) that the calculation would not 


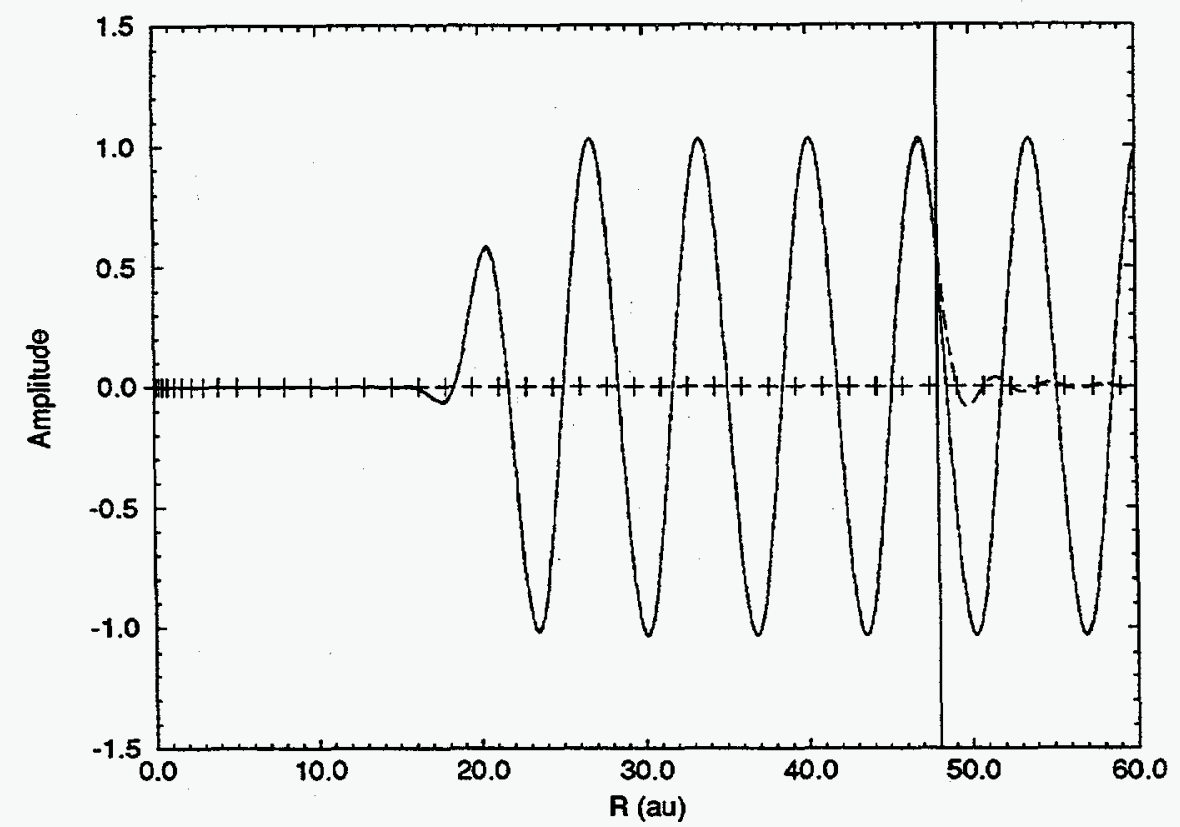

Figure 4.10: Projection of $F_{\mathbf{n} i}$ for $\mathbf{n}=1$ and $i=1$ for $E=0.88 \mathrm{Ryd}$ (45 $\hat{x}$ eigenstates). Solid line is analytical $F_{\mathbf{n} i}$; dashed line is the projection approximation. Plus signs indicate radial DVR points and the long vertical line indicates the end of the extended radial DVR grid.

be very sensitive to small variations in these values. As a result, only the parameter determining the number of terms associated with the reciprocal $R$ expansion $\left(p_{\max }\right)$ was varied. Convergence tests were performed for five important parameters, which are described here:

- $\alpha$ : the multiplicative strength parameter associated with the strengthened static 2s potential $\tilde{V}_{2 s}$ defined by (3.29). The converged calculation uses the value $\alpha=80$.

- $n_{\gamma}$ : the number of Gauss-Legendre DVR points used in the angular degree of freedom. The converged calculation uses the value $n_{\gamma}=4$.

- $R_{\max }$ : determines the value of the scattering coordinate associated with the radial DVR point located furthest from the nucleus. Convergence tests associated with this parameter also vary the positions of the furthest extended radial DVR point (used in the calculation of the bound-free matrix elements) and the fur- 
thest reach of the primitive Sturmian functions such that the furthest extended radial DVR point is 13 au from the furthest radial DVR point actually used in the Hamiltonian inversion, and the zero of the highest-index Sturmian is set to be 13 au further still. The converged calculation uses the value $R_{\max }=35$ au.

- $p_{\max }$ : the absolute value of the most negative $R$ exponent present in the reciprocal $R$ expansion which defines the free functions in (3.103). The converged calculation uses the value $p_{\max }=8$.

- $n_{R}^{e}$ : the number of eigenvalues of the strengthened static $2 \mathrm{~s}$ potential $\tilde{V}_{2 s}$ used in the diagonalization of the position operator $\tilde{x}$. This parameter and $R_{\max }$ therefore determine the density of radial DVR points used in the calculation. The superscript " $e$ " indicates that it is not strictly equal to the number of radial DVR points used, but instead indicates the number of eigenvalues used in their calculation. The converged calculation uses the value $n_{R}^{e}=50$.

Because the transition probability matrix $\mathbf{P}$ (where $P_{i j}=\left|\mathbf{S}_{i j}\right|^{2}$ ) is symmetric, the following convergence plots only show transition probabilities for $\phi_{1 s} \rightarrow \phi_{1 s}$, $\phi_{1 s} \rightarrow \phi_{2 s}, \phi_{1 s} \rightarrow \phi_{2 p}, \phi_{2 s} \rightarrow \phi_{2 s}, \phi_{2 s} \rightarrow \phi_{2 p}$, and $\phi_{2 p} \rightarrow \phi_{2 p}$ transitions. It will be immediately noticed that the energy scale in these convergence plots is identical, but that the probability scale varies widely in order to show the most detail possible. The vertical line in all these convergence plots indicates the $n=3$ transition threshold, while the beginning of the energy scale indicates the $n=2$ threshold.

A few general features in these convergence plots are worth noting. First, it is immediately obvious that the amount of mixing between the $\phi_{1 s}$ state and the two $n=2$ states $\left(\phi_{2 s}\right.$ and $\left.\phi_{2 p}\right)$ is quite small. Second, the mixing between the $\phi_{2 s}$ and $\phi_{2 p}$ states is very strong and energy-dependent between the $n=2$ and $n=3$ thresholds. Third, there is an obvious resonance structure located just above 0.86 Ryd which manifests itself in nearly all the convergence studies.

Convergence of transition probabilities between the three open channels $\phi_{1 s}$, $\phi_{2 s}$, and $\phi_{2 p}$ with respect to the $\alpha$ strength parameter are examined in Figures 4.11 through 4.16. (The convention is introduced in which the $\phi_{1 s}, \phi_{2 s}$, and $\phi_{2 p}$ states are 
referred to by the indices " 1 ", "2", and "3" respectively.) Here, stronger strength parameters $\alpha$ have the effect of skewing the placement of radial DVR points so as to place a higher density of them closer to the origin, while slightly decreasing their density in the asymptotic region of the scattering coordinate. As one would expect

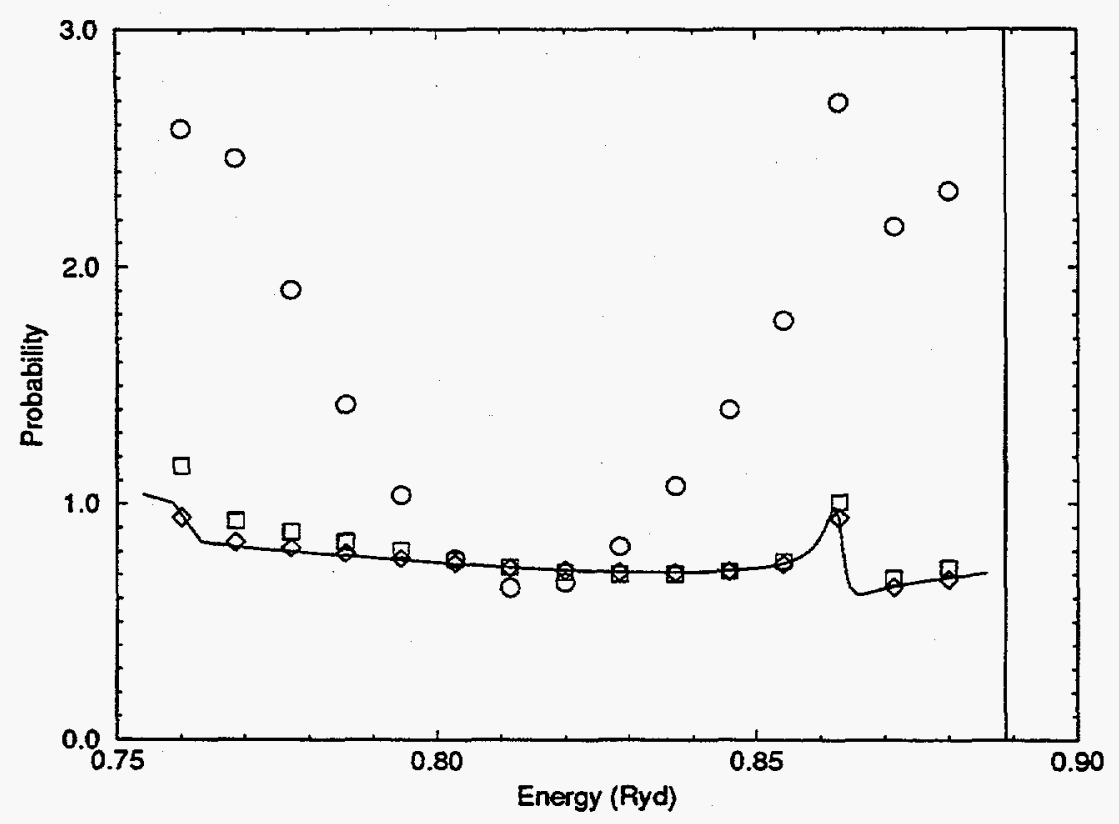

Figure 4.11: $\alpha$ convergence of $\phi_{1 s} \rightarrow \phi_{1 s}$ transition probability $P_{11}$. Circle: $\alpha=10$; Square: $\alpha=30$; Diamond: $\alpha=50$; Solid line: $\alpha=80$. Vertical line is $n=3$ threshold.

from the particle densities of the $\phi_{1 s}, \phi_{2 s}$, and $\phi_{2 p}$ hydrogen states, transition probabilities which involve scattering wavefunctions with large $\phi_{1 s}$ components require a high density of radial DVR points near the origin, and are mostly likely to exhibit slow convergence with respect to this parameter. This is clearly demonstrated in Figures 4.11 through 4.13 , in which the transition probabilities out of the $\phi_{1 s}$ hydrogen state are almost universally incorrect throughout the energy region. Interestingly, the resonance structure just above 0.86 Ryd still manifests itself even when the density of radial DVR points near the origin is not particularly high.

Not surprisingly, the transition probabilities between the $\phi_{2 s}$ and $\phi_{2 p}$ states converge much more rapidly with respect to this parameter. As mentioned previously, this is in all likelihood due to the fact that the total scattering wavefunction in such 


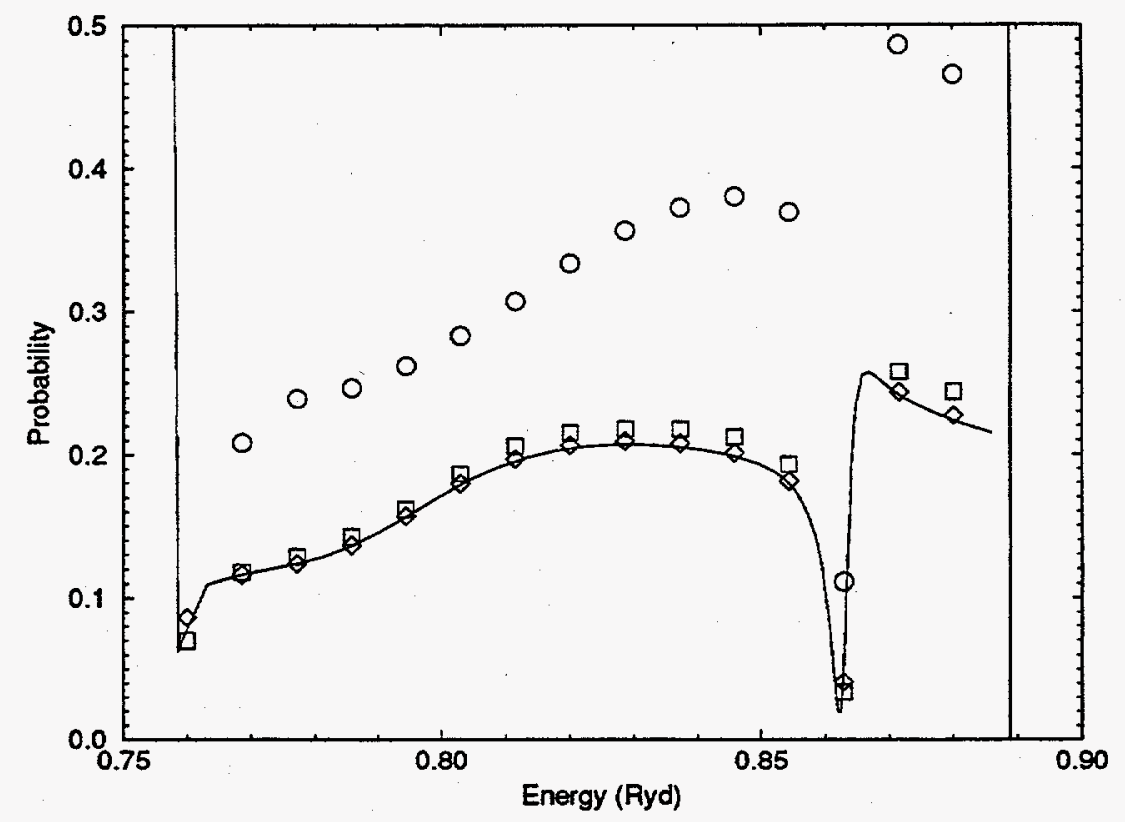

Figure 4.12: $\alpha$ convergence of $\phi_{1 s} \rightarrow \phi_{2 s}$ transition probability $P_{12}$. Circle: $\alpha=10$; Square: $\alpha=30$; Diamond: $\alpha=50$; Solid line: $\alpha=80$. Vertical line is $n=3$ threshold.

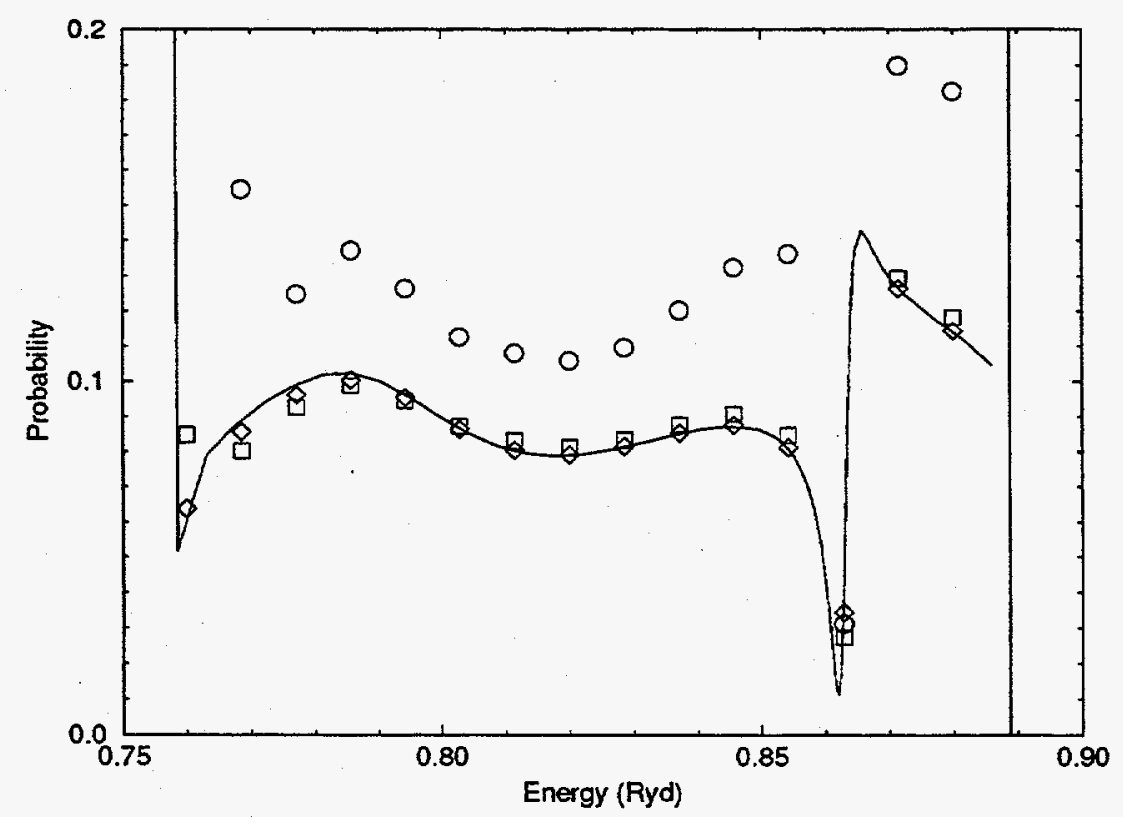

Figure 4.13: $\alpha$ convergence of $\phi_{1 s} \rightarrow \phi_{2 p}$ transition probability $P_{13}$. Circle: $\alpha=10$; Square: $\alpha=30$; Diamond: $\alpha=50$; Solid line: $\alpha=80$. Vertical line is $n=3$ threshold. 


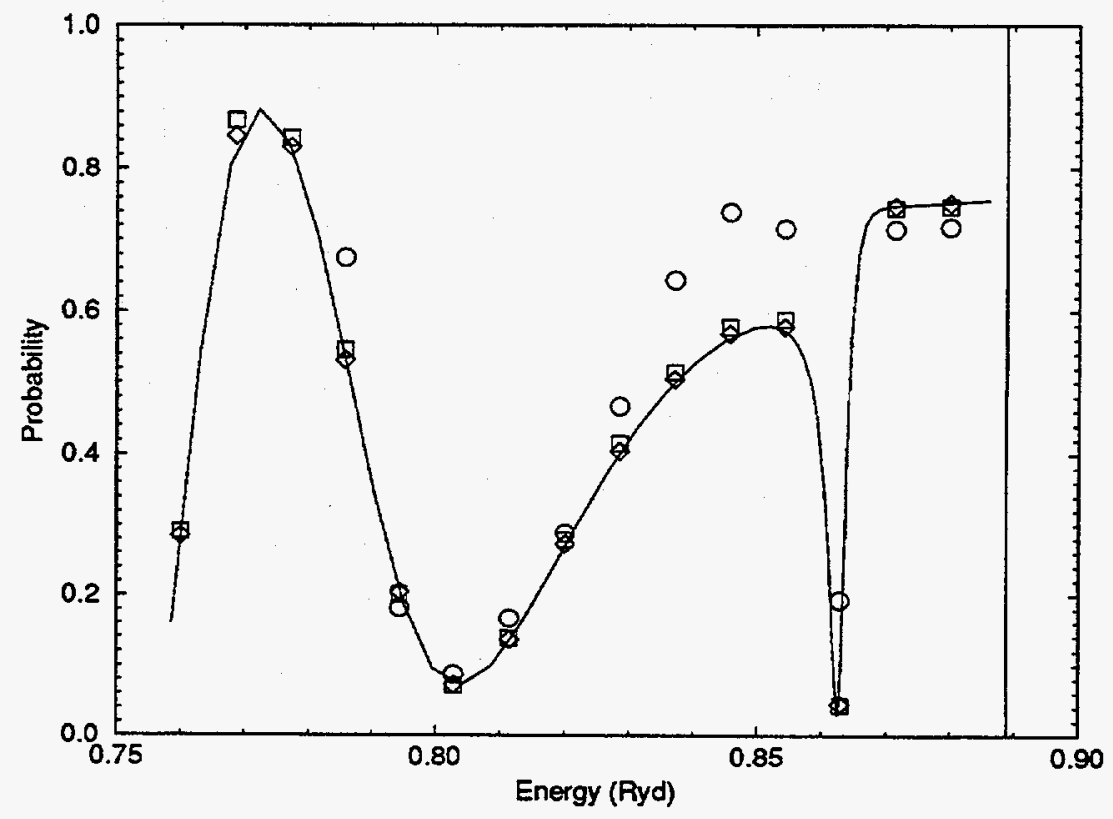

Figure 4.14: $\alpha$ convergence of $\phi_{2 s} \rightarrow \phi_{2 s}$ transition probability $P_{22}$. Circle: $\alpha=10$; Square: $\alpha=30$; Diamond: $\alpha=50$; Solid line: $\alpha=80$. Vertical line is $n=3$ threshold.

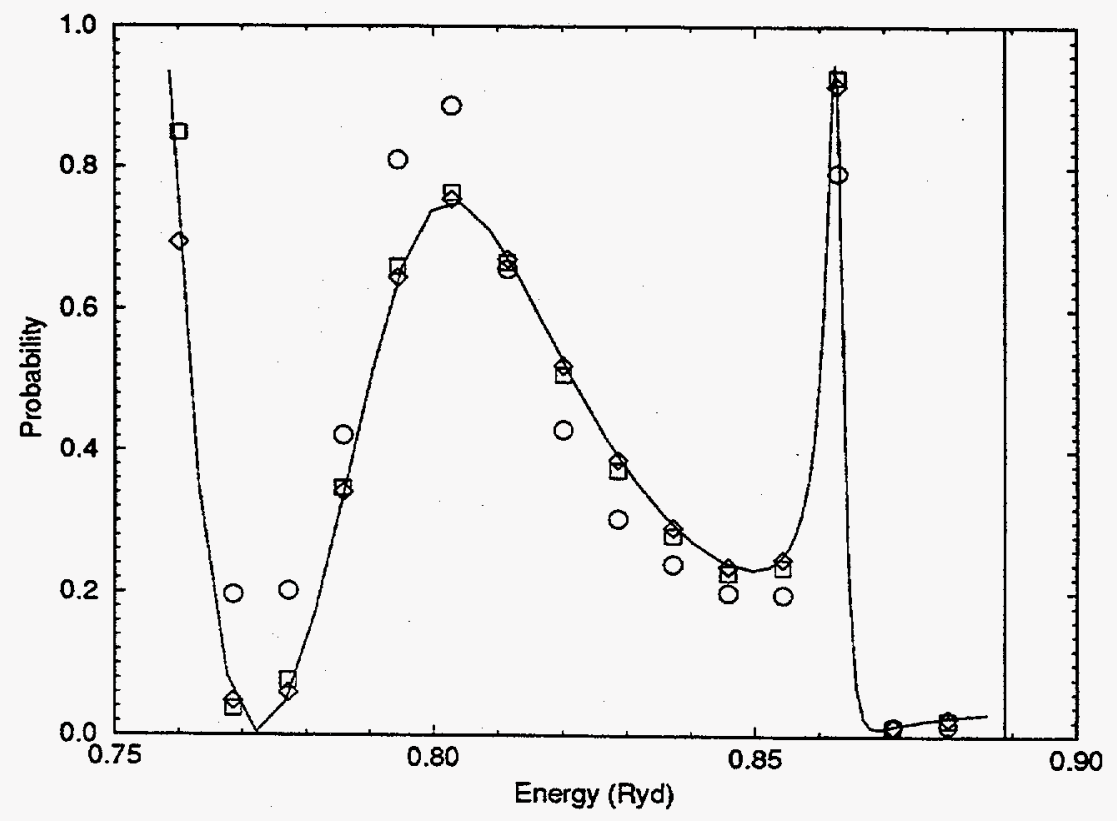

Figure 4.15: $\alpha$ convergence of $\phi_{2 s} \rightarrow \phi_{2 p}$ transition probability $P_{23}$. Circle: $\alpha=10$; Square: $\alpha=30$; Diamond: $\alpha=50$; Solid line: $\alpha=80$. Vertical line is $n=3$ threshold. 


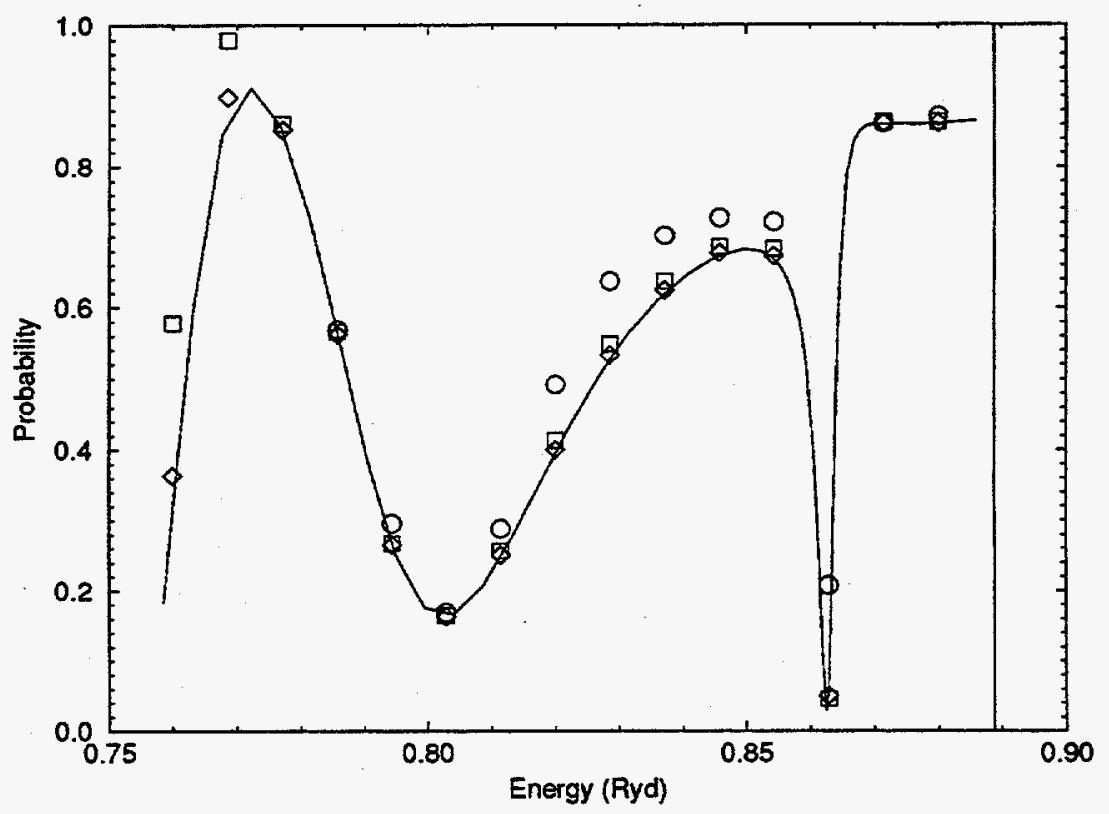

Figure 4.16: $\alpha$ convergence of $\phi_{2 p} \rightarrow \phi_{2 p}$ transition probability $P_{33}$. Circle: $\alpha=10$; Square: $\alpha=30$; Diamond: $\alpha=50$; Solid line: $\alpha=80$. Vertical line is $n=3$ threshold.

transitions has a very small projection into the $\phi_{1 s}$ state and therefore very few radial DVR points are needed to accurately represent the wavefunction.

One curious feature present in all six of the transitions represented by Figures 4.11 through 4.16 is the fact that errors incurred as a result of not having an adequate density of radial DVR points near the origin appear to overestimate the transition probability, regardless of energy. It is also evident that convergence is stronger nearer to the $n=3$ threshold than near the $n=2$ threshold. In fact, the ability of the calculation to attain quantitative results just above the $n=2$ threshold is demonstrably weaker than anywhere else in this particular energy region.

The reasons for this have been discussed elsewhere by Schwartz [18] and Burke and Schey [89]. The ability of the reciprocal $R$ expansion to adequately represent the scattering free functions at low kinetic energies is not very good. As a result, the interaction region becomes quite large and the limited extent of the radial DVR becomes a serious liability as it is no longer able to compensate for the weakness of the reciprocal $R$ expansion. 
A similar problem is avoided just below the $n=3$ threshold. In this part of the energy spectrum difficulties usually arise from the fact that the exponential tails on the projection of the scattering wavefunction onto the closed $n=3$ channels become very long and therefore move outside of the interaction region. But the implementation of this particular radial DVR enables a better treatment of larger interaction regions by virtue of the fact that the density of such points becomes relatively constant for scattering regions beyond a particular value of $R$, unlike densities associated with standard Gaussian quadrature DVRs. This results in an ability to place the radial DVR points more efficiently, and therefore to use them to span a larger interaction region. (As will be seen later in the section dealing with the convergence of the individual $\mathbf{S}$ matrix elements, the exponential tails of the radial wavefunction still pose difficulties at energies very close to the $n=3$ threshold, but these difficulties are minimized with the present method.)

The convergence of singlet transition probabilities with respect to the number of angular Gauss-Legendre DVR points $n_{\gamma}$ in demonstrated in Figures 4.17 through 4.22. Not surprisingly, the most rapid convergence is demonstrated for the $\phi_{1 s} \rightarrow \phi_{1 s}$ transition. Once again, the lack of mixing between the $\phi_{1 s}$ and other hydrogen states permits this scattering wavefunction to be accurately represented by fewer angular DVR points. This must in large part be due to the spherical symmetry of the strongest bound state component. It will be noticed that convergence of the $\phi_{2 s} \rightarrow \phi_{2 s}$ transition shown in Figure 4.20 is not nearly as strong, in spite of the spherical symmetry of the $\phi_{2 s}$ state. This is very likely due to the strong projection of the associated scattering wavefunction into the strongly mixing $\phi_{2 p}$ state, which does not have spherical symmetry. As a general rule, convergence of all transition probabilities is adequate with merely three Gauss-Legendre angular DVR points, with rather good convergence being attained with four.

One of the most striking features of these particular convergence studies is the almost complete lack of any resonance structure near 0.86 Ryd when only one angular DVR point is employed, even in Figure 4.17 which shows convergence of the $\phi_{1 s} \rightarrow \phi_{1 s}$ transition probability. This is clear evidence that while spherical symmetry obviously dominates the scattering wavefunction in the asymptotic region 


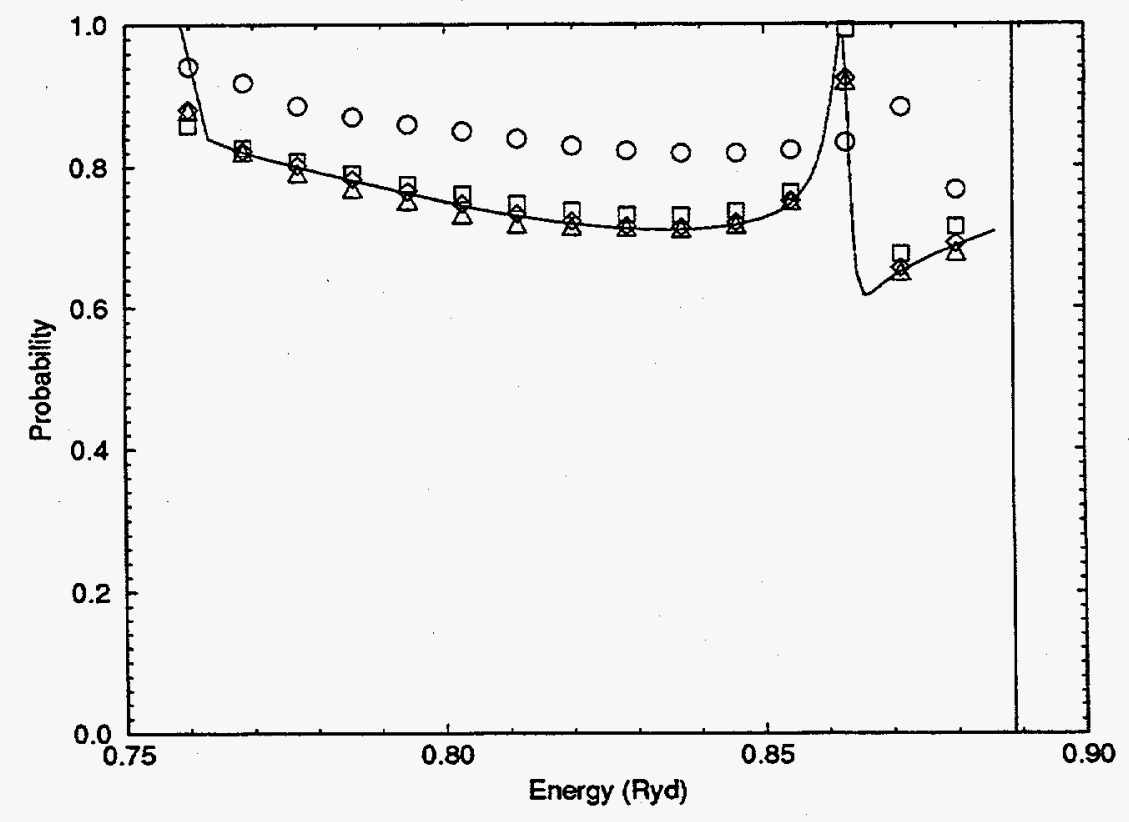

Figure 4.17: $n_{\gamma}$ convergence of $\phi_{1 s} \rightarrow \phi_{1 s}$ transition probability $P_{11}$. Circle: $n_{\gamma}=1$; Square: $n_{\gamma}=2$; Diamond: $n_{\gamma}=3$; Solid line: $n_{\gamma}=4$; Up triangle: $n_{\gamma}=5$. Vertical line is $n=3$ threshold.

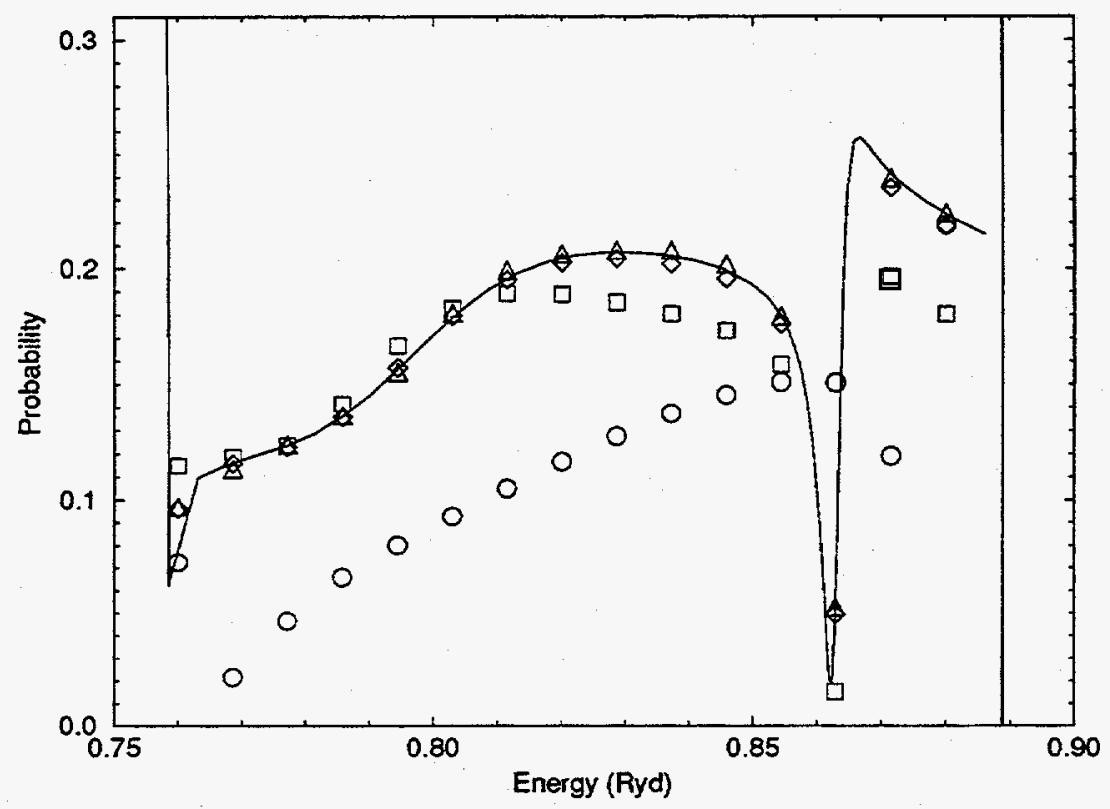

Figure 4.18: $n_{\gamma}$ convergence of $\phi_{1 s} \rightarrow \phi_{2 s}$ transition probability $P_{12}$. Circle: $n_{\gamma}=1$; Square: $n_{\gamma}=2$; Diamond: $n_{\gamma}=3$; Solid line: $n_{\gamma}=4$; Up triangle: $n_{\gamma}=5$. Vertical line is $n=3$ threshold. 


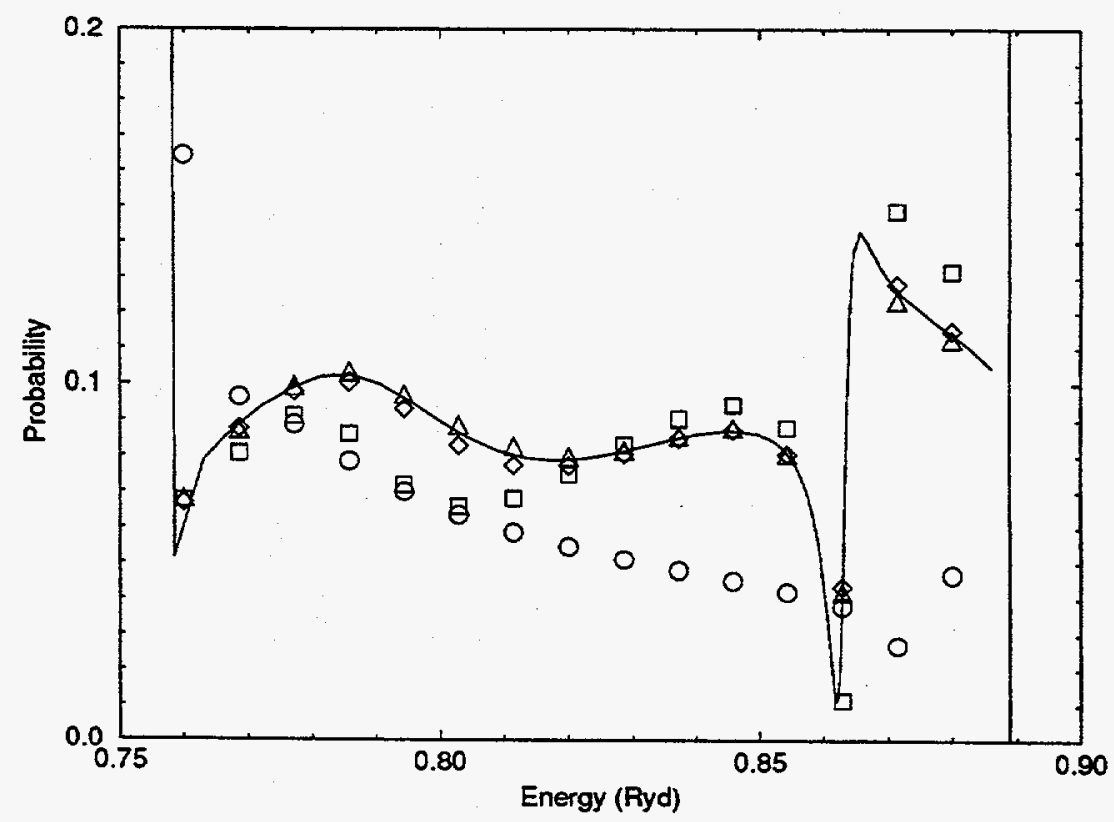

Figure 4.19: $n_{\gamma}$ convergence of $\phi_{1 s} \rightarrow \phi_{2 p}$ transition probability $P_{13}$. Circle: $n_{\gamma}=1$; Square: $n_{\gamma}=2$; Diamond: $n_{\gamma}=3$; Solid line: $n_{\gamma}=4$; Up triangle: $n_{\gamma}=5$. Vertical line is $n=3$ threshold.

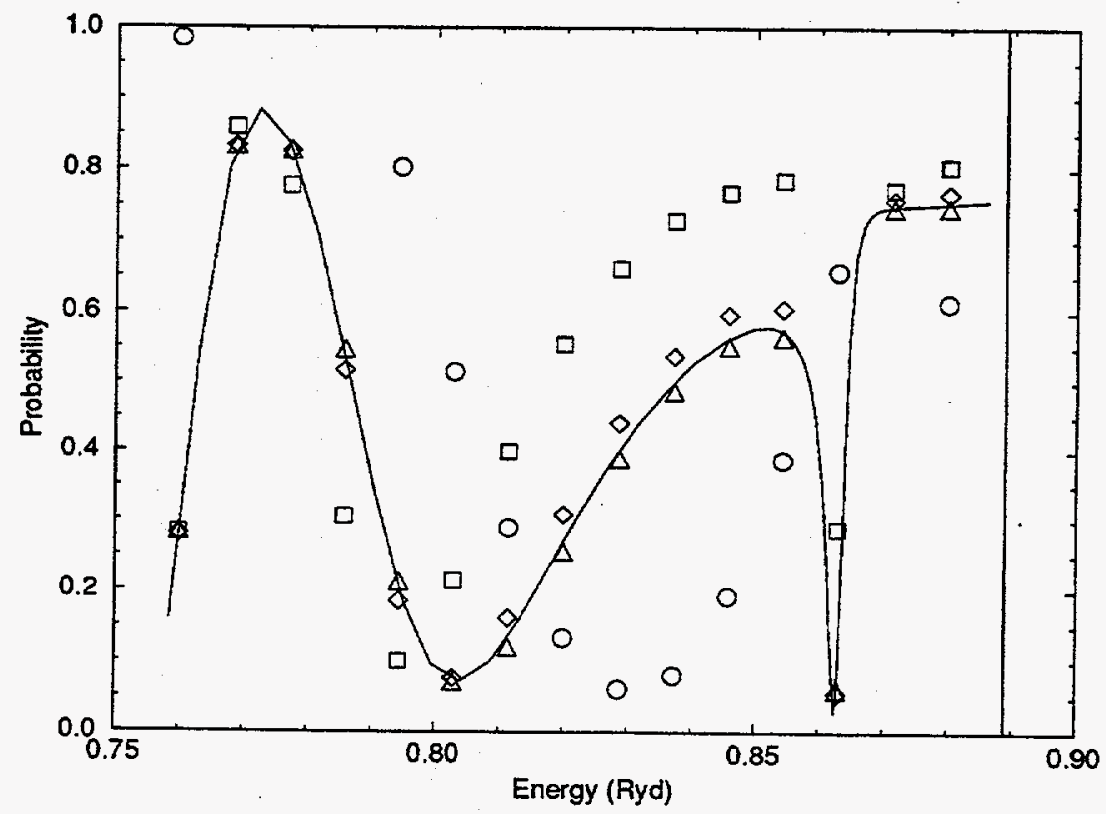

Figure 4.20: $n_{\gamma}$ convergence of $\phi_{2 s} \rightarrow \phi_{2 s}$ transition probability $P_{22}$. Circle: $n_{\gamma}=1$; Square: $n_{\gamma}=2$; Diamond: $n_{\gamma}=3$; Solid line: $n_{\gamma}=4 ;$ Up triangle: $n_{\gamma}=5$. Vertical line is $n=3$ threshold. 


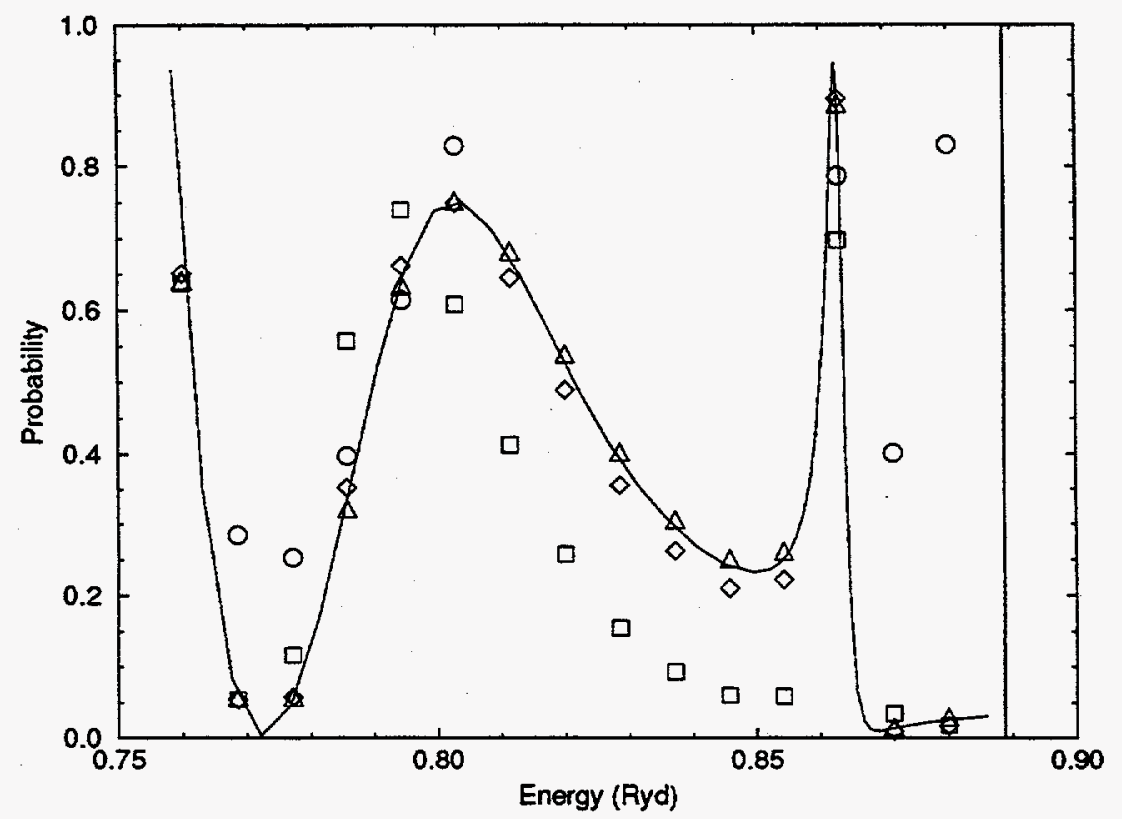

Figure 4.21: $n_{\gamma}$ convergence of $\phi_{2 s} \rightarrow \phi_{2 p}$ transition probability $P_{23}$. Circle: $n_{\gamma}=1$; Square: $n_{\gamma}=2$; Diamond: $n_{\gamma}=3$; Solid line: $n_{\gamma}=4$; Up triangle: $n_{\gamma}=5$. Vertical line is $n=3$ threshold.

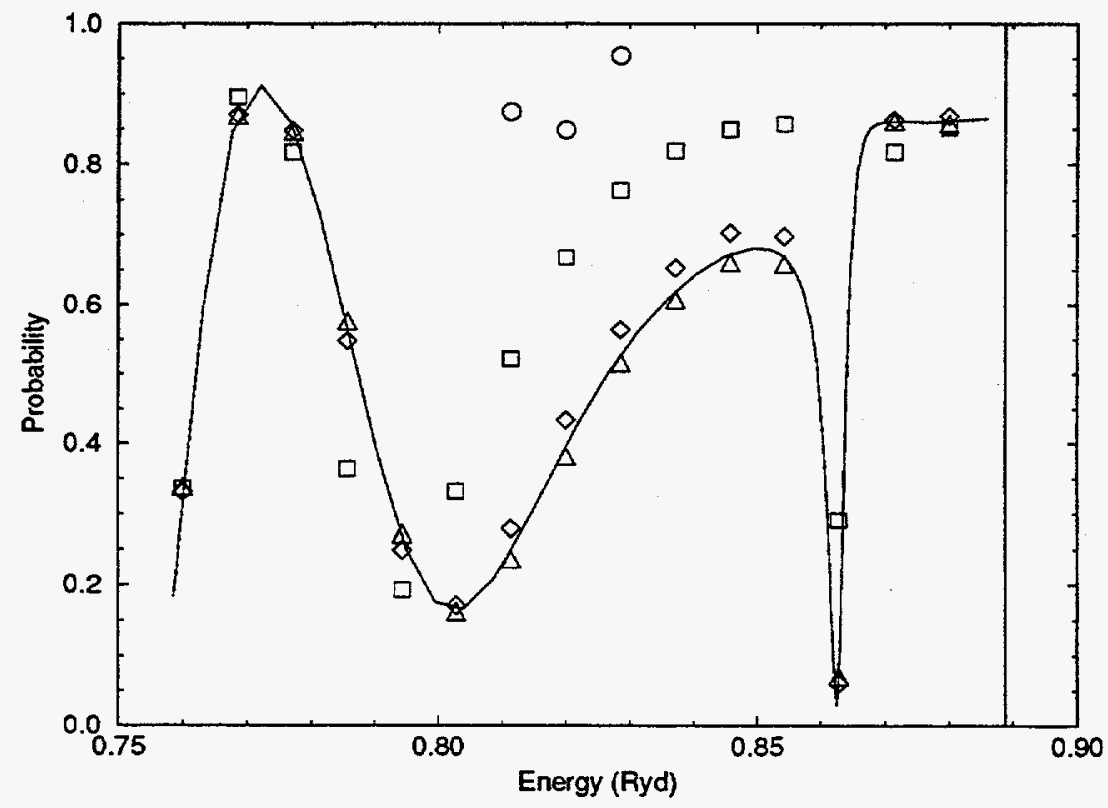

Figure 4.22: $n_{\gamma}$ convergence of $\phi_{2 p} \rightarrow \phi_{2 p}$ transition probability $P_{33}$. Circle: $n_{\gamma}=1$; Square: $n_{\gamma}=2$; Diamond: $n_{\gamma}=3$; Solid line: $n_{\gamma}=4$; Up triangle: $n_{\gamma}=5$. Vertical line is $n=3$ threshold. 
of the radial coordinate, such is not the case within the interaction region where interelectron repulsion evidently distorts this symmetry. Inclusion of two angular DVR points is sufficient to cause at least a slight manifestation of the resonance behavior in all of the transition probabilities. The weakest convergence is demonstrated in Figure 4.22 , but full convergence appears to be attained with only four angular DVR points.

Convergence of singlet transition probabilities with respect to the position of the last radial DVR point $R_{\max }$ is shown in Figure 4.23 through Figure 4.28. Here $R_{\max }$ represents the position of the center of the radial DVR function located

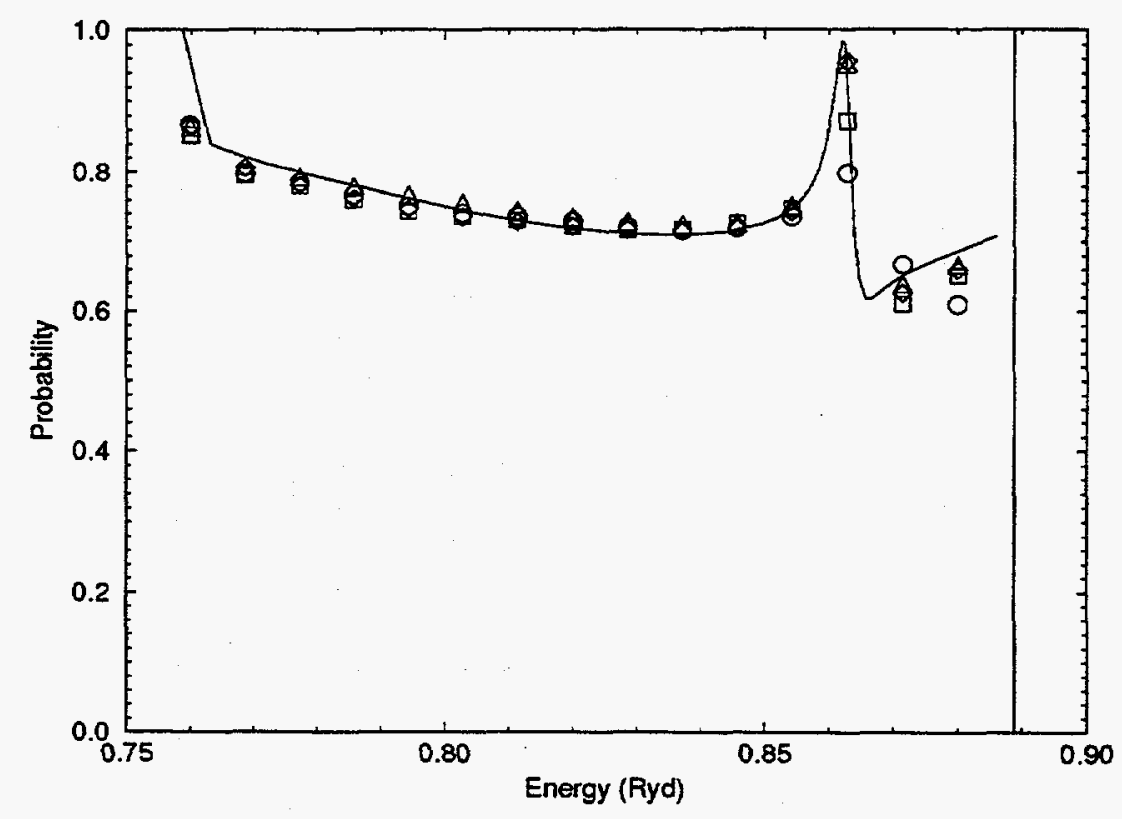

Figure 4.23: $R_{\max }$ convergence of $\phi_{1 s} \rightarrow \phi_{1 s}$ transition probability $P_{11}$. Circle: $R_{\max }=27$ au; Square: $R_{\max }=29$ au; Diamond: $R_{\max }=31 \mathrm{au} ; \mathrm{Up}$ triangle: $R_{\max }=33$ au; Solid line: $R_{\max }=35$ au. Vertical line is $n=3$ threshold.

furthest from the origin. As such, it is a direct measure of the extent of the interaction region spanned by the $L^{2}$ basis set. The highly localized DVR functions allow one to study the extent of this interaction region in a very physical way, without having to interpret results in terms of projections onto spatially diffuse functions (such as is required when conventional delocalized $L^{2}$ basis functions are used).

The most noteworthy characteristic present in these particular convergence 


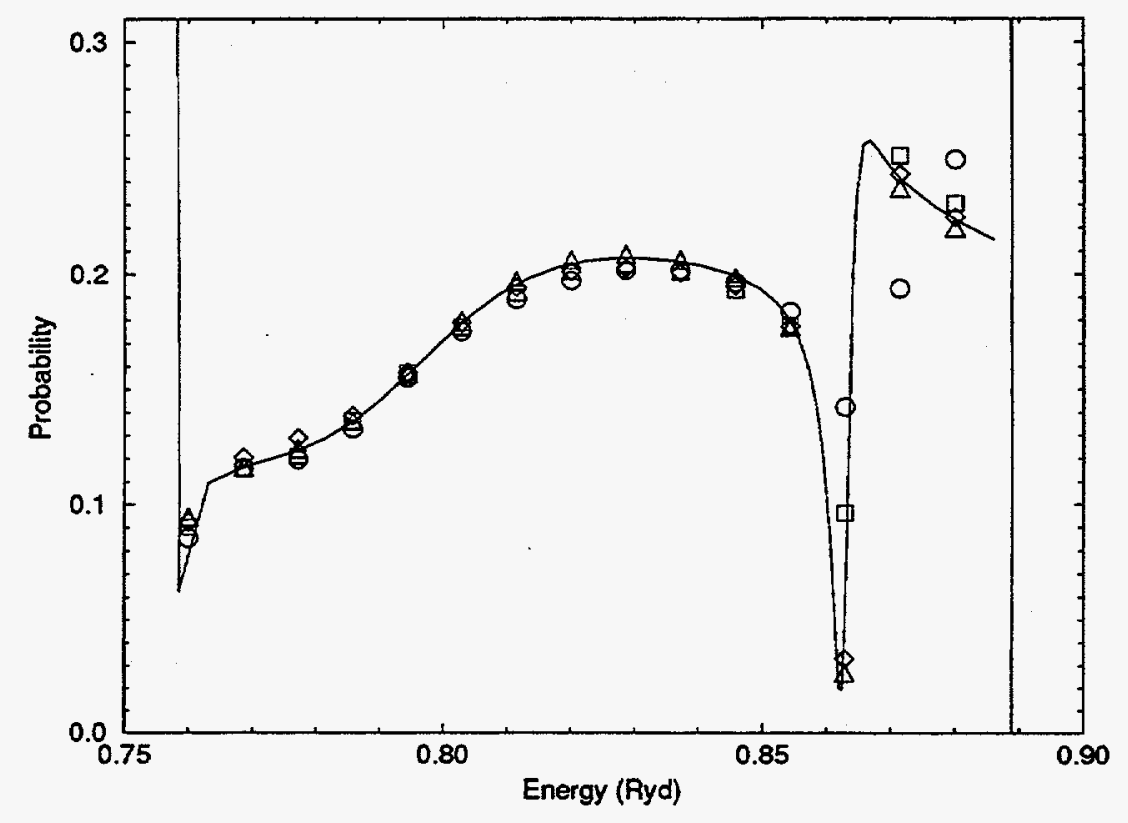

Figure 4.24: $R_{\max }$ convergence of $\phi_{1 s} \rightarrow \phi_{2 s}$ transition probability $P_{12}$. Circle: $R_{\max }=27 \mathrm{au} ;$ Square: $R_{\max }=29 \mathrm{au} ;$ Diamond: $R_{\max }=31 \mathrm{au}$; Up triangle: $R_{\max }=33$ au; Solid line: $R_{\max }=35$ au. Vertical line is $n=3$ threshold.

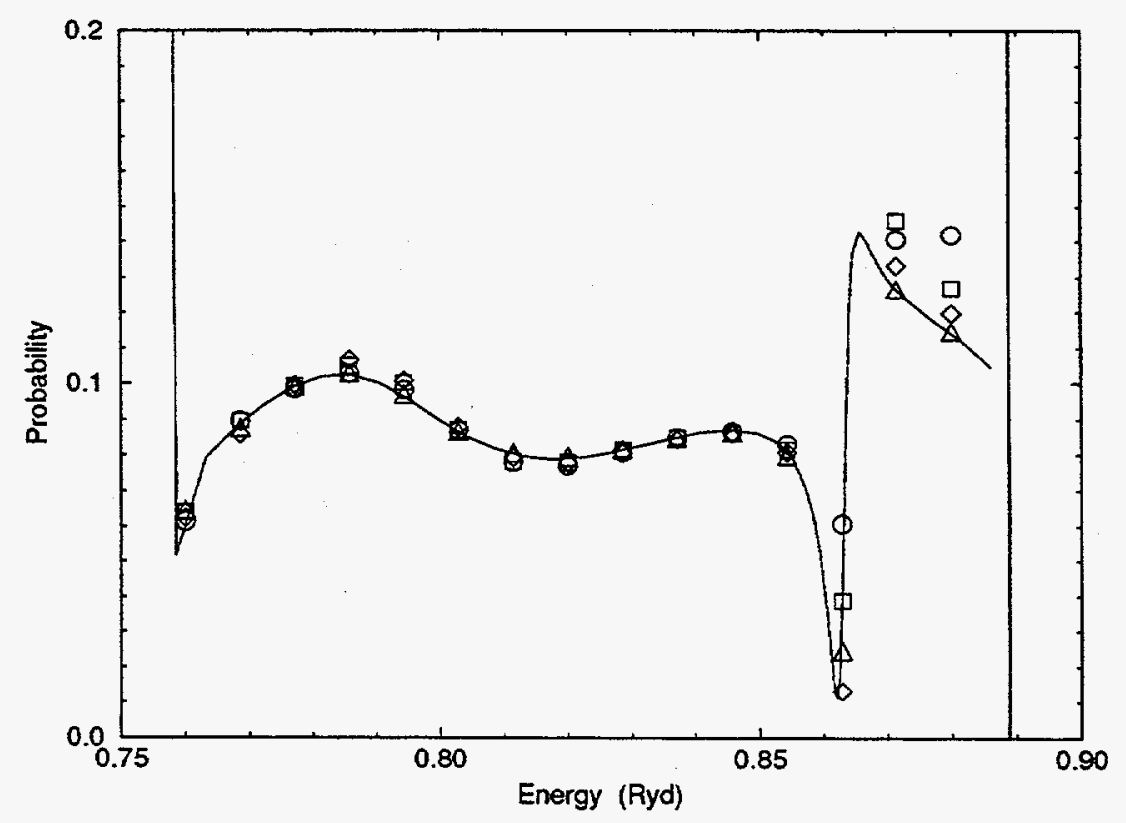

Figure 4.25: $R_{\max }$ convergence of $\phi_{1 s} \rightarrow \phi_{2 p}$ transition probability $P_{13}$. Circle: $R_{\max }=27 \mathrm{au} ;$ Square: $R_{\max }=29 \mathrm{au} ;$ Diamond: $R_{\max }=31 \mathrm{au} ;$ Up triangle: $R_{\max }=33 \mathrm{au}$; Solid line: $R_{\max }=35$ au. Vertical line is $n=3$ threshold. 


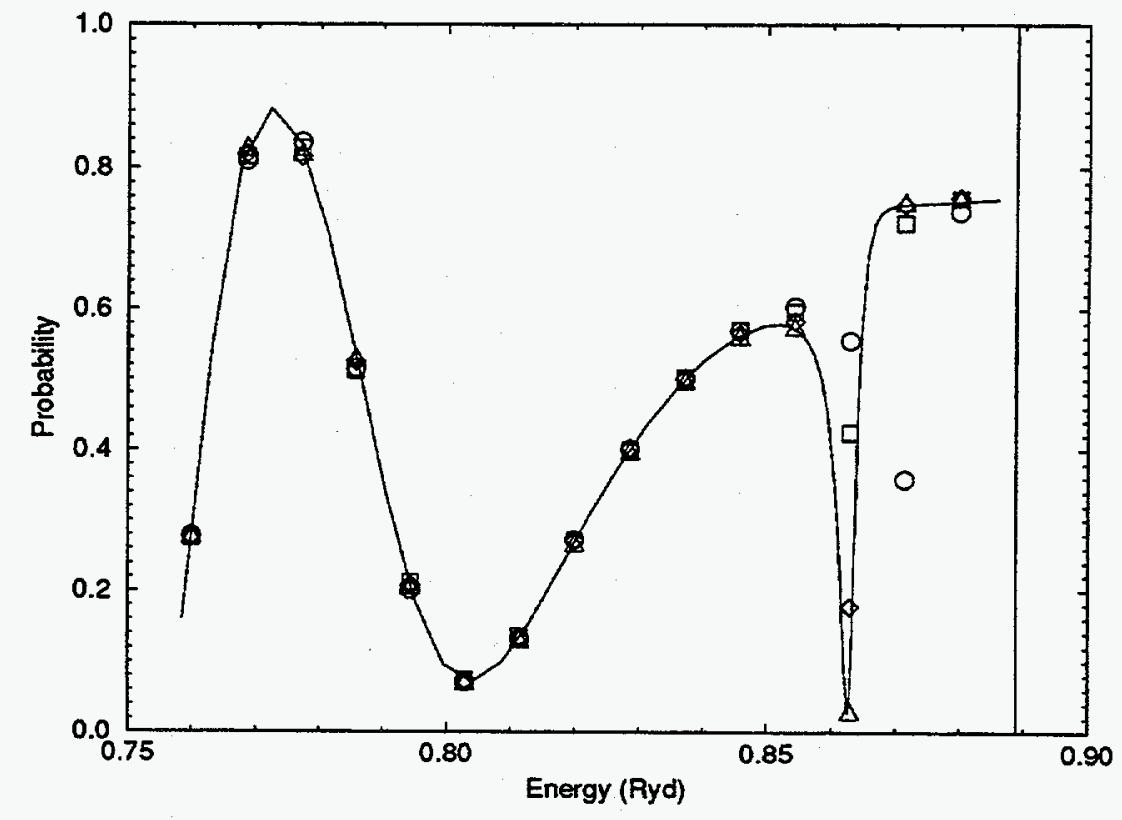

Figure 4.26: $R_{\max }$ convergence of $\phi_{2 s} \rightarrow \phi_{2 s}$ transition probability $P_{22}$. Circle: $R_{\text {max }}=27$ au; Square: $R_{\max }=29$ au; Diamond: $R_{\max }=31$ au; Up triangle: $R_{\max }=33 \mathrm{au}$; Solid line: $R_{\max }=35$ au. Vertical line is $n=3$ threshold.

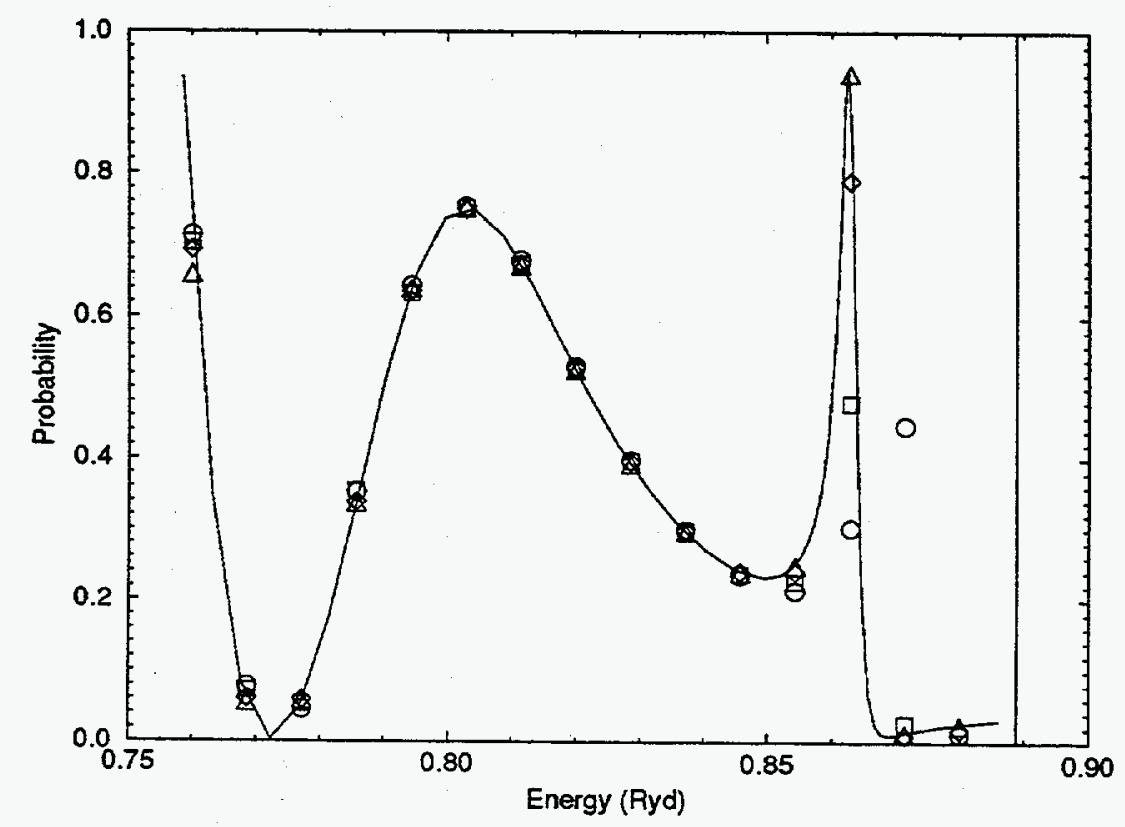

Figure 4.27: $R_{\max }$ convergence of $\phi_{2 s} \rightarrow \phi_{2 p}$ transition probability $P_{23}$. Circle: $R_{\max }=27$ au; Square: $R_{\max }=29$ au; Diamond: $R_{\max }=31$ au; Up triangle: $R_{\max }=33 \mathrm{au}$; Solid line: $R_{\max }=35$ au. Vertical line is $n=3$ threshold. 


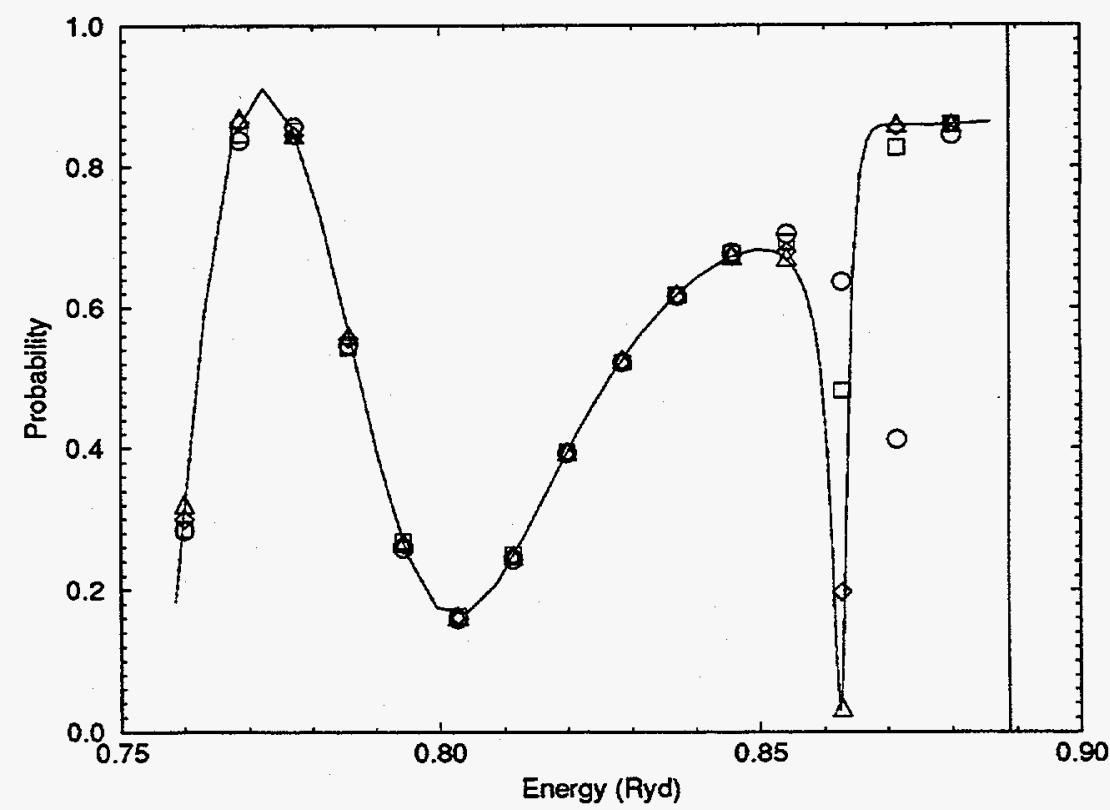

Figure 4.28: $R_{\max }$ convergence of $\phi_{2 p} \rightarrow \phi_{2 p}$ transition probability $P_{33}$. Circle: $R_{\max }=27$ au; Square: $R_{\max }=29$ au; Diamond: $R_{\max }=31$ au; Up triangle: $R_{\max }=33 \mathrm{au}$; Solid line: $R_{\max }=35$ au. Vertical line is $n=3$ threshold.

studies is the fact that (with the exception of energy regions just above the $n=2$ threshold, just below the $n=3$ threshold, and immediately surrounding the resonance at $0.862 \mathrm{Ryd}$ ) the transition probabilities are relatively converged when $R_{\max }=27$ au. The strength and sharpness of this resonance increases steadily until it also becomes converged when $R_{\max }=35$ au. This is most dramatically demonstrated in Figures 4.26 through 4.28 which show transition probabilities between the $\phi_{2 s}$ and $\phi_{2 p}$ hydrogen states. When $R_{\max }=27$ au, the resonance behavior does not seem to manifest itself at all, indicating that the free functions employed in the calculation near this resonance energy are not adequate to represent the scattering wavefunction without more assistance from the $L^{2}$ radial DVR basis set.

Convergence with respect to the $R_{\text {max }}$ parameter is difficult in two other energy regions. For example, significant difficulty is encountered just above the $n=2$ threshold. In fact, even qualitatively correct results are not obtained below 0.76 Ryd. This is not unexpected behavior. The reciprocal $R$ expansion which has been employed in the calculation of the asymptotic form of the free functions has significant 
sine/cosine character far from the origin, as evidenced by (3.103). But when the magnitude of the asymptotic wave vector $k \approx 0$, the physically correct wavefunction behaves more as $R^{-p}$, where $p$ can be as small as zero. This "straightline" behavior of the wavefunction is badly represented by the present sine/cosine expansion, and requires inclusion of terms of the form

$$
\gamma_{p}^{\mathbf{n} n n} R^{-p} \quad \text { for } p=0 \rightarrow \infty .
$$

where $\mathbf{n}$ indicates the barely open channel. (Terms of this kind are discussed by Schwartz [18] and Burke and Schey [89].) But a non-zero coefficient cannot be assigned to the $p=0$ term (unlike the $\alpha_{0}^{\mathbf{n} n n}$ and $\beta_{0}^{\mathbf{n} n n}$ terms). They therefore cannot be included in the present recurrence expansion, which requires a non-zero starting coefficient. Another way to look at this behavior is to realize that when $k \approx 0$, the range of influence of the bound hydrogen atom on the wavefunction of the scattering electron increases, thereby requiring non-conventional asymptotic forms. A standard open-channel Numerov propagation would probably provide better results here since it imposes no functional form on the asymptotic wavefunction.

Convergence is also slow just below the $n=3$ threshold. Scattering energies just below a threshold require a diffuse $L^{2}$ basis in order to represent the slow decay of the exponential wavefunctions associated with the negative kinetic energies of the nearby closed channel. Once again, this causes an effective increase in the range of the interaction region, and the value of $R_{\max }$ in the present technique must therefore increase to prohibitively large values.

Convergence of singlet transition probabilities with respect to the number of free function reciprocal $R$ expansion terms $p_{\max }$ is examined in Figures 4.29 through 4.34. It is noticed that convergence of the transition probabilities involving the $\phi_{1 s}$ state is quite good regardless of the number of terms included in the expansion of the free function. Even a simple expansion in which no reciprocal powers of $R$ are included (equivalent to a simple free particle wavefunction) gives good results and still demonstrates resonance behavior near 0.86 Ryd.

The importance of additional terms in the reciprocal $R$ expansion is most clearly demonstrated in the transition probabilities between the $\phi_{2 s}$ and $\phi_{2 p}$ states. 


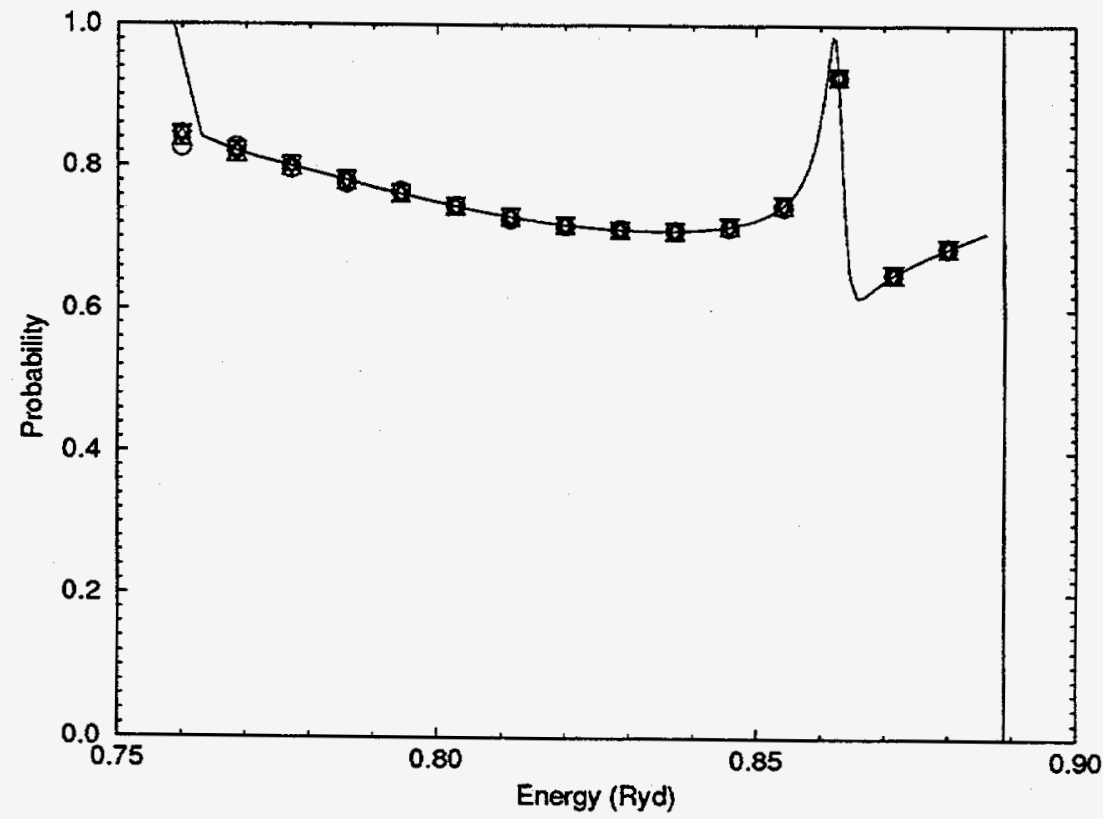

Figure 4.29: $p_{\max }$ convergence of $\phi_{1 s} \rightarrow \phi_{1 s}$ transition probability $P_{11}$. Circle: $p_{\max }=$ 0; Square: $p_{\max }=1$; Diamond: $p_{\max }=2$; Up triangle: $p_{\max }=3$; Down triangle: $p_{\max }=4$; Solid line: $p_{\max }=8$. Vertical line is $n=3$ threshold.

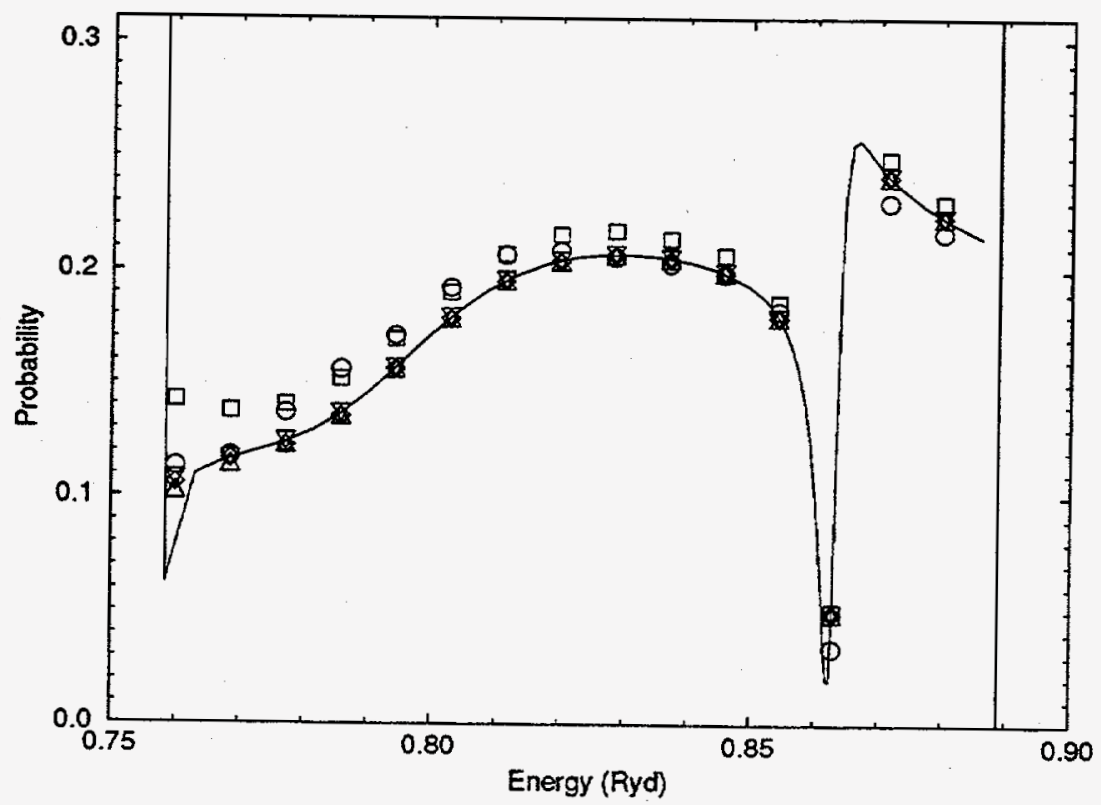

Figure 4.30: $p_{\max }$ convergence of $\phi_{1 s} \rightarrow \phi_{2 s}$ transition probability $P_{12}$. Circle: $p_{\max }=$ 0; Square: $p_{\max }=1$; Diamond: $p_{\max }=2$; Up triangle: $p_{\max }=3$; Down triangle: $p_{\max }=4$; Solid line: $p_{\max }=8$. Vertical line is $n=3$ threshold. 


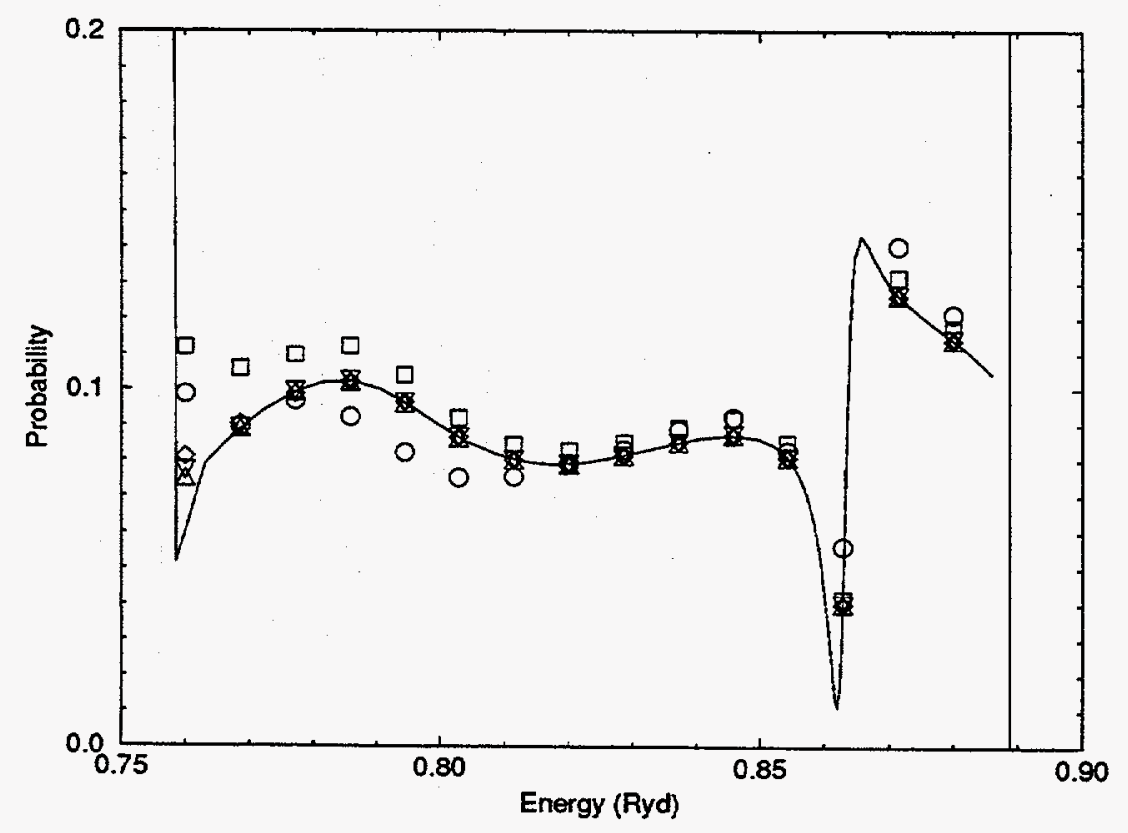

Figure 4.31: $p_{\max }$ convergence of $\phi_{1 s} \rightarrow \phi_{2 p}$ transition probability $P_{13}$. Circle: $p_{\max }=$ 0 ; Square: $p_{\max }=1$; Diamond: $p_{\max }=2$; Up triangle: $p_{\max }=3$; Down triangle: $p_{\max }=4$; Solid line: $p_{\max }=8$. Vertical line is $n=3$ threshold.

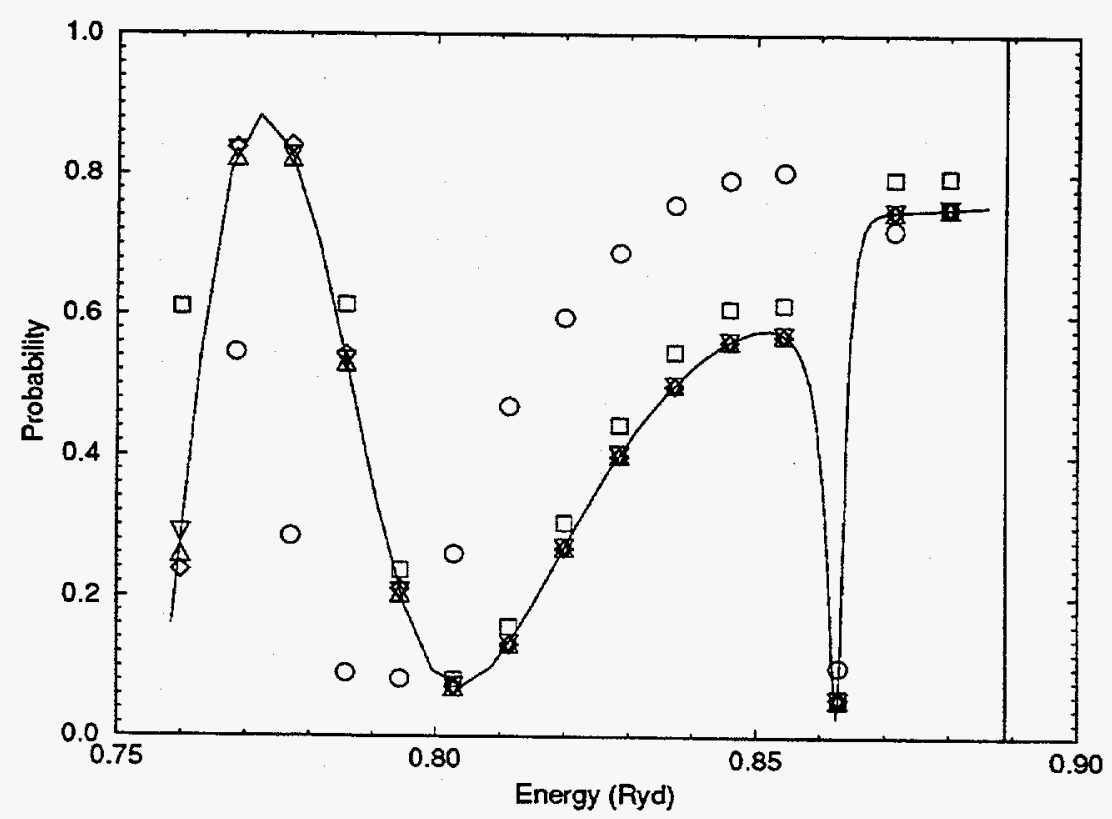

Figure 4.32: $p_{\max }$ convergence of $\phi_{2 s} \rightarrow \phi_{2 s}$ transition probability $P_{22}$. Circle: $p_{\max }=$ 0; Square: $p_{\max }=1$; Diamond: $p_{\max }=2$; Up triangle: $p_{\max }=3$; Down triangle: $p_{\max }=4$; Solid line: $p_{\max }=8$. Vertical line is $n=3$ threshold. 


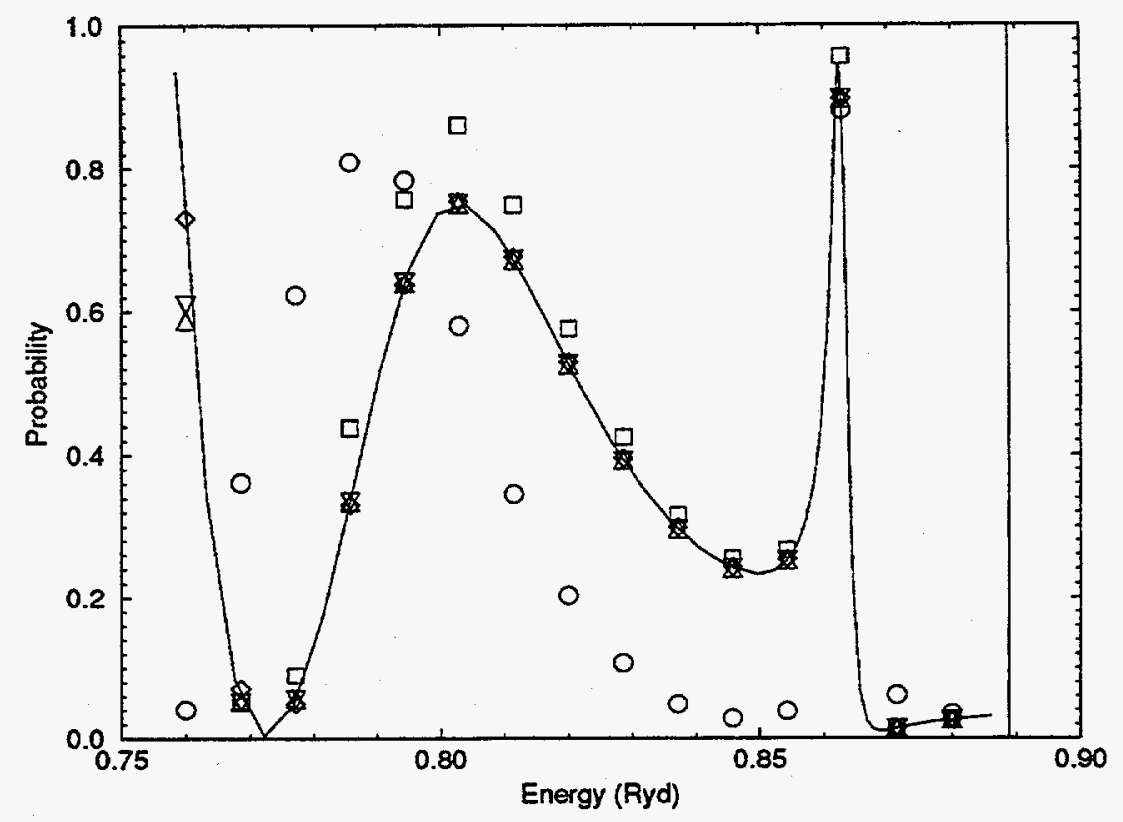

Figure 4.33: $p_{\max }$ convergence of $\phi_{2 s} \rightarrow \phi_{2 p}$ transition probability $P_{23}$. Circle: $p_{\max }=$ 0; Square: $p_{\max }=1$; Diamond: $p_{\max }=2$; Up triangle: $p_{\max }=3$; Down triangle: $p_{\max }=4$; Solid line: $p_{\max }=8$. Vertical line is $n=3$ threshold.

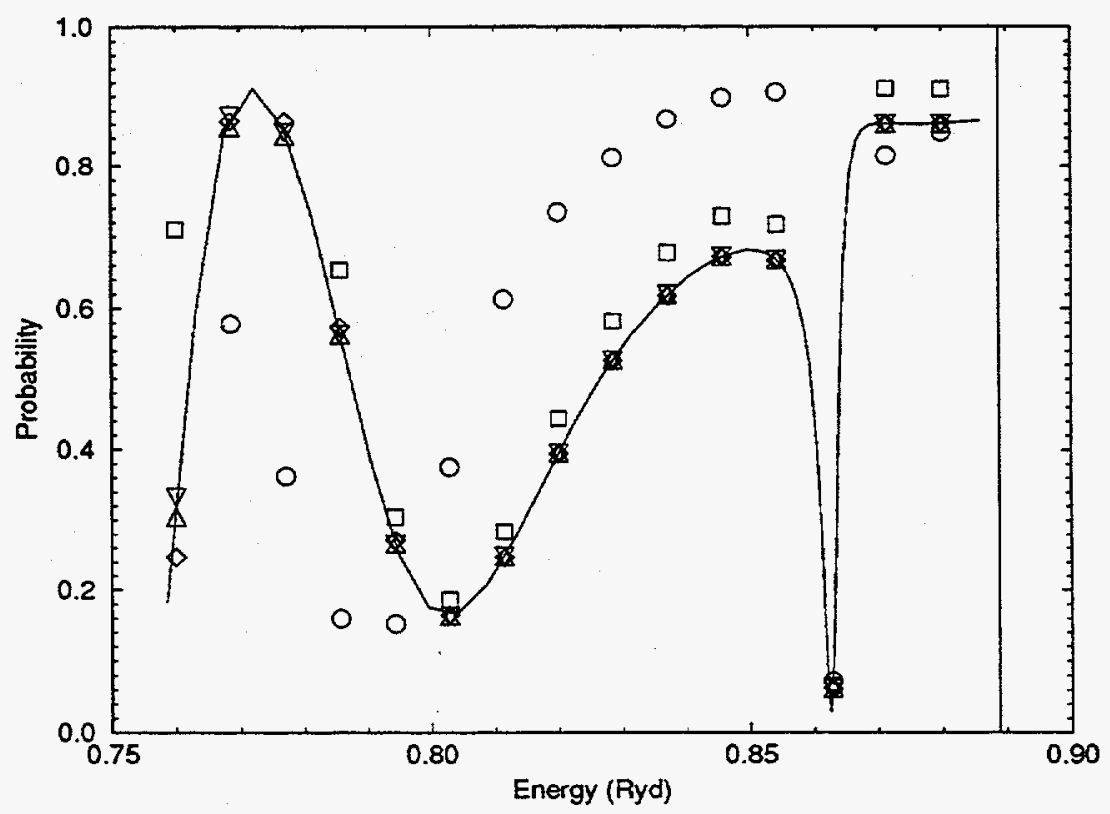

Figure 4.34: $p_{\max }$ convergence of $\phi_{2 p} \rightarrow \phi_{2 p}$ transition probability $P_{33}$. Circle: $p_{\max }=$ 0; Square: $p_{\max }=1$; Diamond: $p_{\max }=2$; Up triangle: $p_{\max }=3$; Down triangle: $p_{\max }=4$; Solid line: $p_{\max }=8$. Vertical line is $n=3$ threshold. 
Here the dipole moment coupling between these two states demands the additional terms of the expansion, and it is seen that leaving them out gives a very poor result. Inclusion of just the first non-zero power of $R$ is still not sufficient to attain truly converged results. Although, the resonance behavior near 0.86 Ryd is still quite evident. And as before in previous convergence studies, convergence of the scattering calculation at very low asymptotic kinetic energies is difficult, due to the inability of the reciprocal $R$ expansion to adequately represent the free function with simple sine and cosine functions, thereby requiring an increase in the size of the interaction region spanned by the $L^{2}$ functions.

Convergence with respect to $n_{R}^{e}$ (the number of eigenvalues of the strengthened static 2s potential $\tilde{V}_{2 s}$ used in the diagonalization of the position operator $\hat{\mathbf{x}}$ ) is demonstrated in Figures 4.35 through 4.40. The parameter $n_{R}^{e}$ does not lend itself easily to a simple physical interpretation, but it is roughly proportional to the density of radial DVR points (with all other parameters held constant). Convergence with respect to this parameter is fairly typical. The resonance near $0.86 \mathrm{Ryd}$ is visible at most of the DVR point densities represented, and convergence appears to be slowest just above the $n=2$ threshold. Interpretation of this particular convergence study must be done cautiously since alteration of the value of $n_{R}^{e}$ causes changes in every region of the scattering coordinate within the interaction region, both close to the origin (where representation of the $\phi_{1 s}$ function is most likely to be affected) and at the end of the interaction region (where representation of high kinetic energy free functions are mostly likely to be affected).

\subsubsection{Convergence of Singlet S Matrices}

In this section the convergence of the $\mathbf{S}$ matrix elements for singlet scattering is discussed. This gives a more complete picture of the convergence process since the $S$ matrix contains ALL information about the asymptotic state of the scattering wavefunction in the form of both an amplitude and complex phase associated with a particular bound state. (The transition probability only provides a value for the amplitude.) 


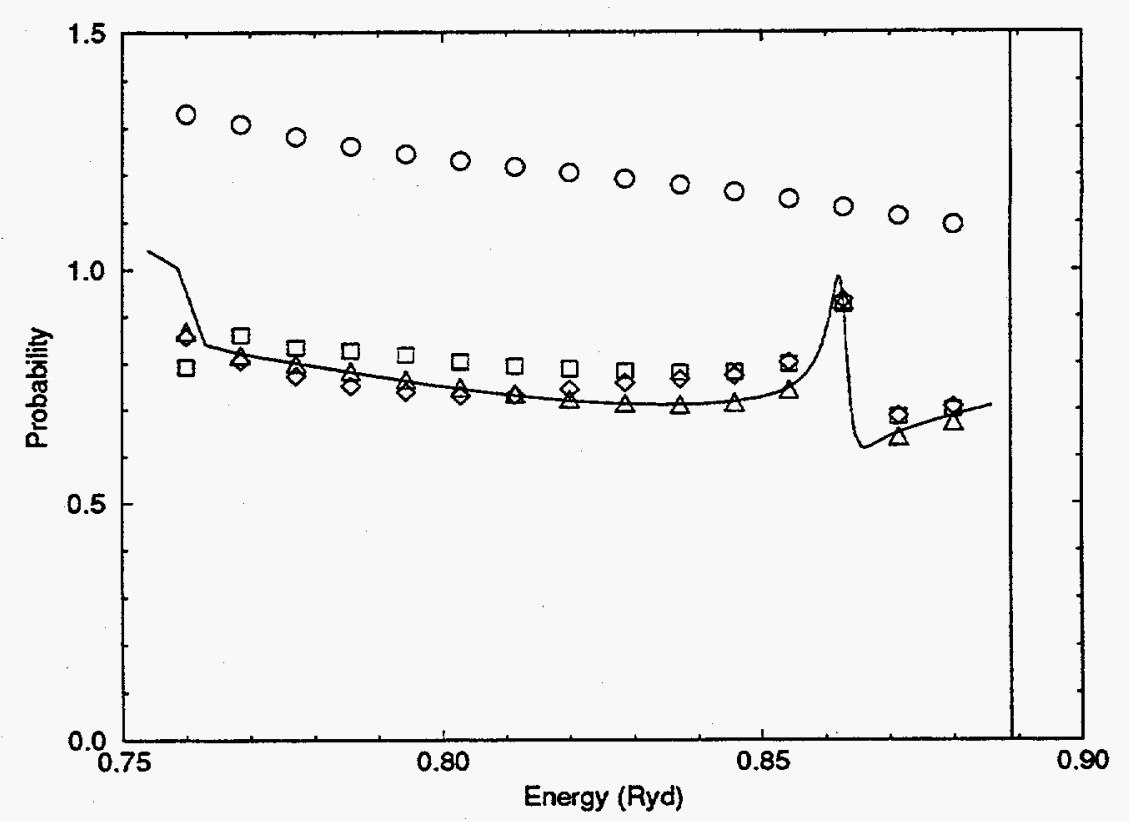

Figure 4.35: $n_{R}^{e}$ convergence of $\phi_{1 s} \rightarrow \phi_{1 s}$ transition probability $P_{11}$. Circle: $n_{R}^{e}=30$; Square: $n_{R}^{e}=35$; Diamond: $n_{R}^{e}=40$; Up triangle: $n_{R}^{e}=45$; Solid line: $n_{R}^{e}=50$. Vertical line is $n=3$ threshold.

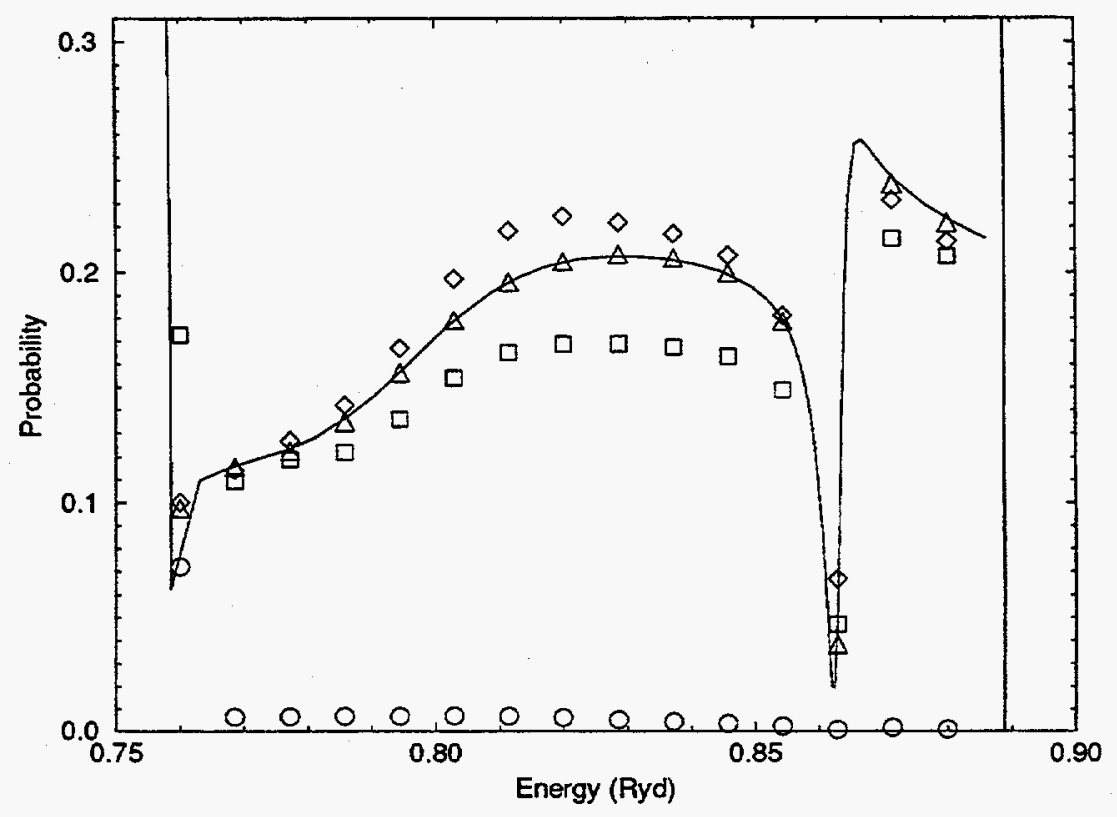

Figure 4.36: $n_{R}^{e}$ convergence of $\phi_{1 s} \rightarrow \phi_{2 s}$ transition probability $P_{12}$. Circle: $n_{R}^{e}=30$; Square: $n_{R}^{e}=35$; Diamond: $n_{R}^{e}=40$; Up triangle: $n_{R}^{e}=45$; Solid line: $n_{R}^{e}=50$. Vertical line is $n=3$ threshold. 


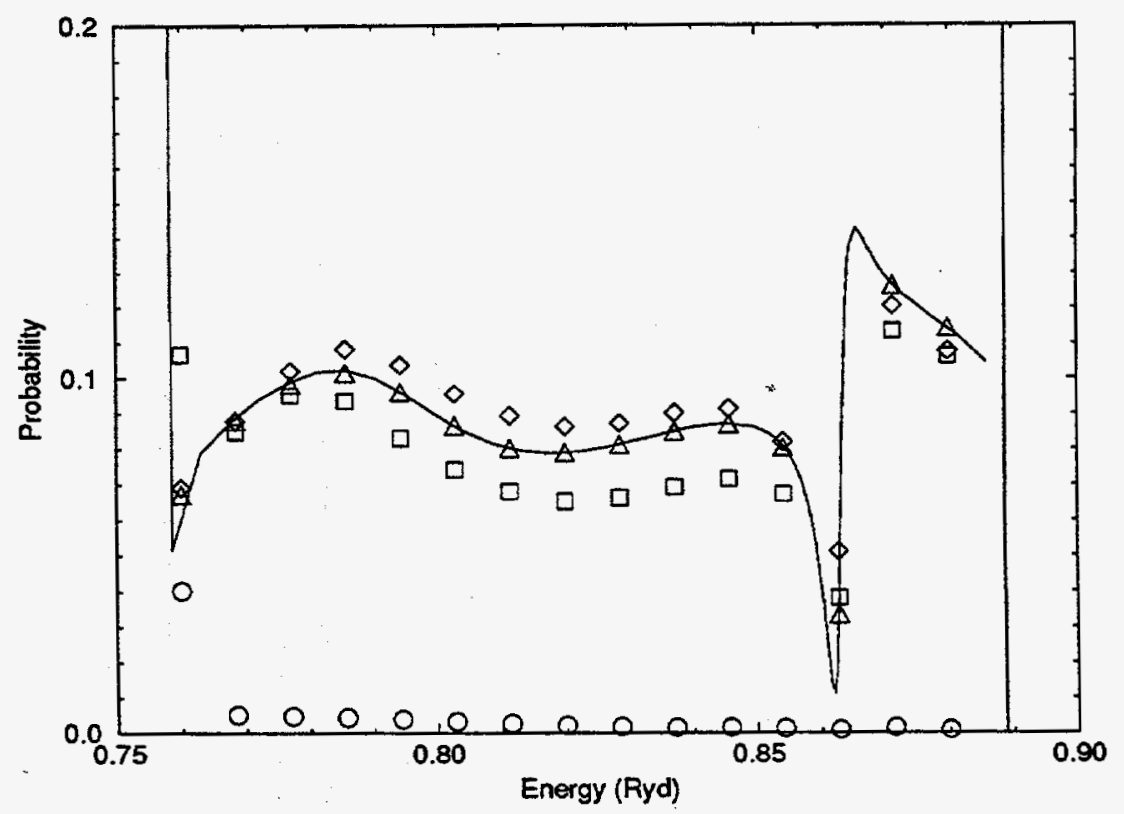

Figure 4.37: $n_{R}^{e}$ convergence of $\phi_{1 s} \rightarrow \phi_{2 p}$ transition probability $P_{13}$. Circle: $n_{R}^{e}=30$; Square: $n_{R}^{e}=35$; Diamond: $n_{R}^{e}=40$; Up triangle: $n_{R}^{e}=45$; Solid line: $n_{R}^{e}=50$. Vertical line is $n=3$ threshold.

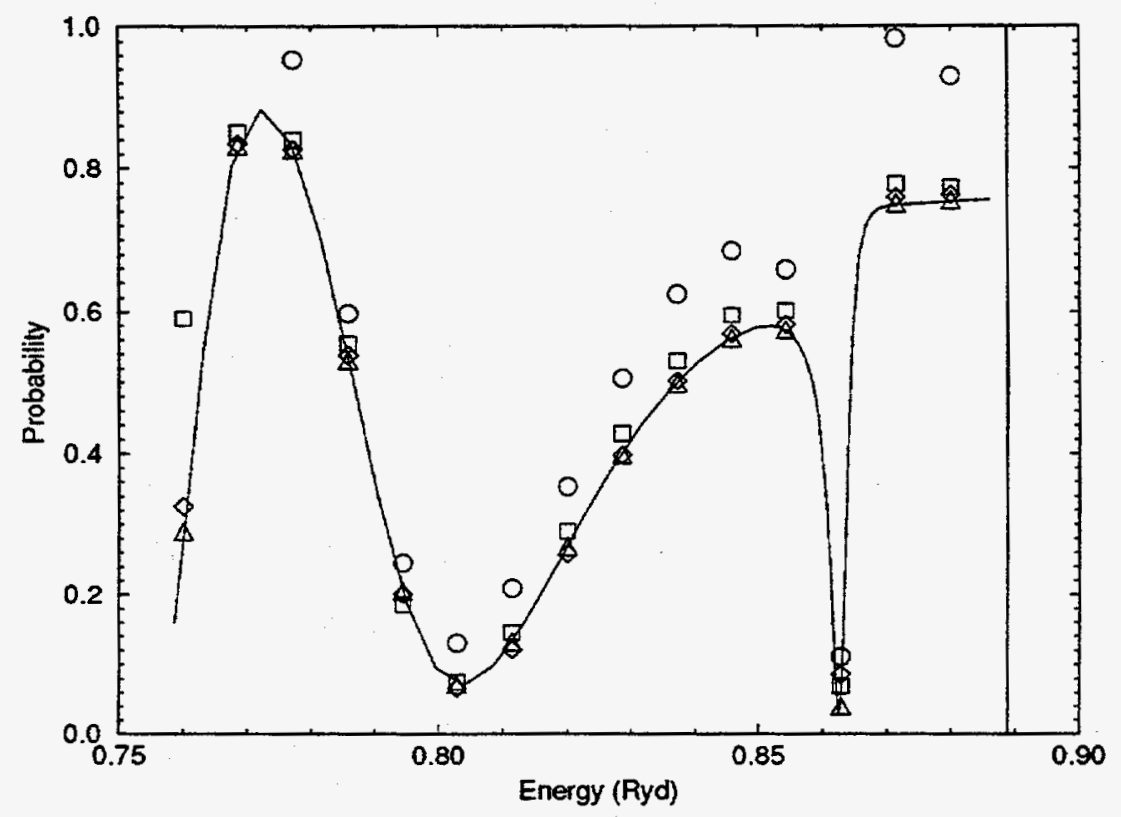

Figure 4.38: $n_{R}^{e}$ convergence of $\phi_{2 s} \rightarrow \phi_{2 s}$ transition probability $P_{22}$. Circle: $n_{R}^{e}=30$; Square: $n_{R}^{e}=35$; Diamond: $n_{R}^{e}=40$; Up triangle: $n_{R}^{e}=45$; Solid line: $n_{R}^{e}=50$. Vertical line is $n=3$ threshold. 


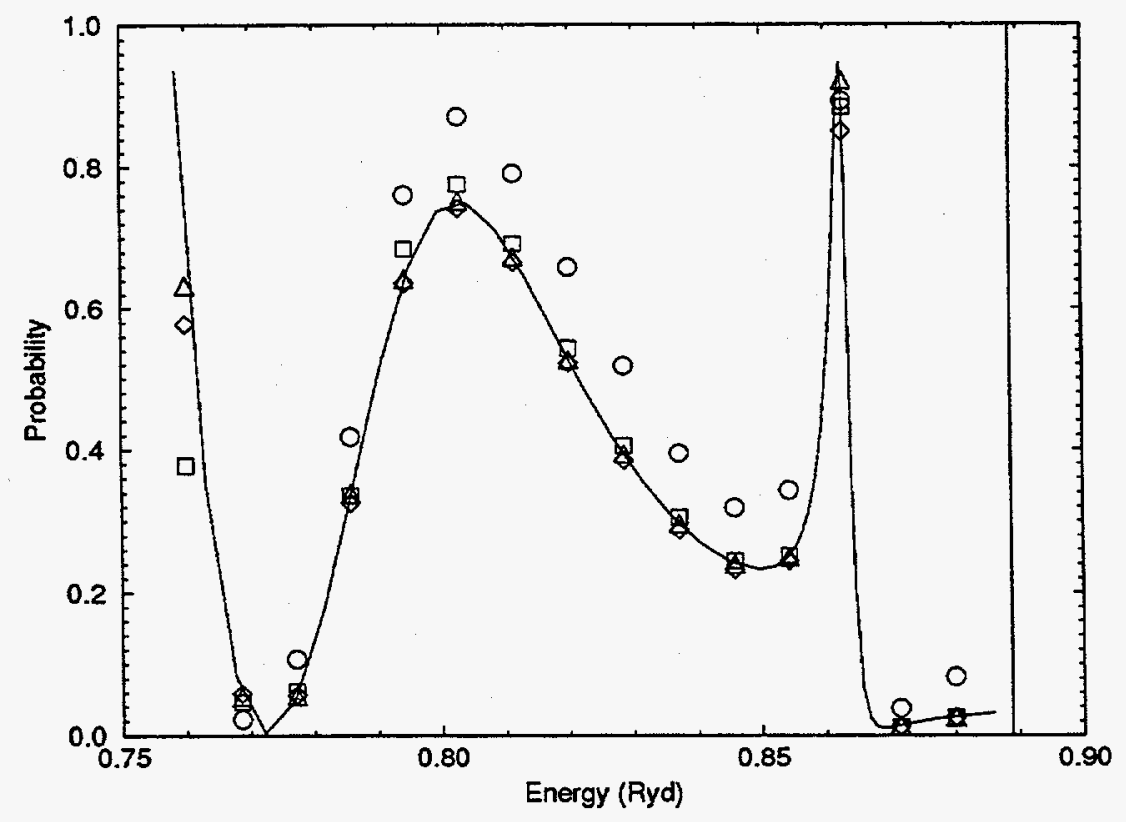

Figure 4.39: $n_{R}^{e}$ convergence of $\phi_{2 s} \rightarrow \phi_{2 p}$ transition probability $P_{23}$. Circle: $n_{R}^{e}=30$; Square: $n_{R}^{e}=35$; Diamond: $n_{R}^{e}=40$; Up triangle: $n_{R}^{e}=45$; Solid line: $n_{R}^{e}=50$. Vertical line is $n=3$ threshold.

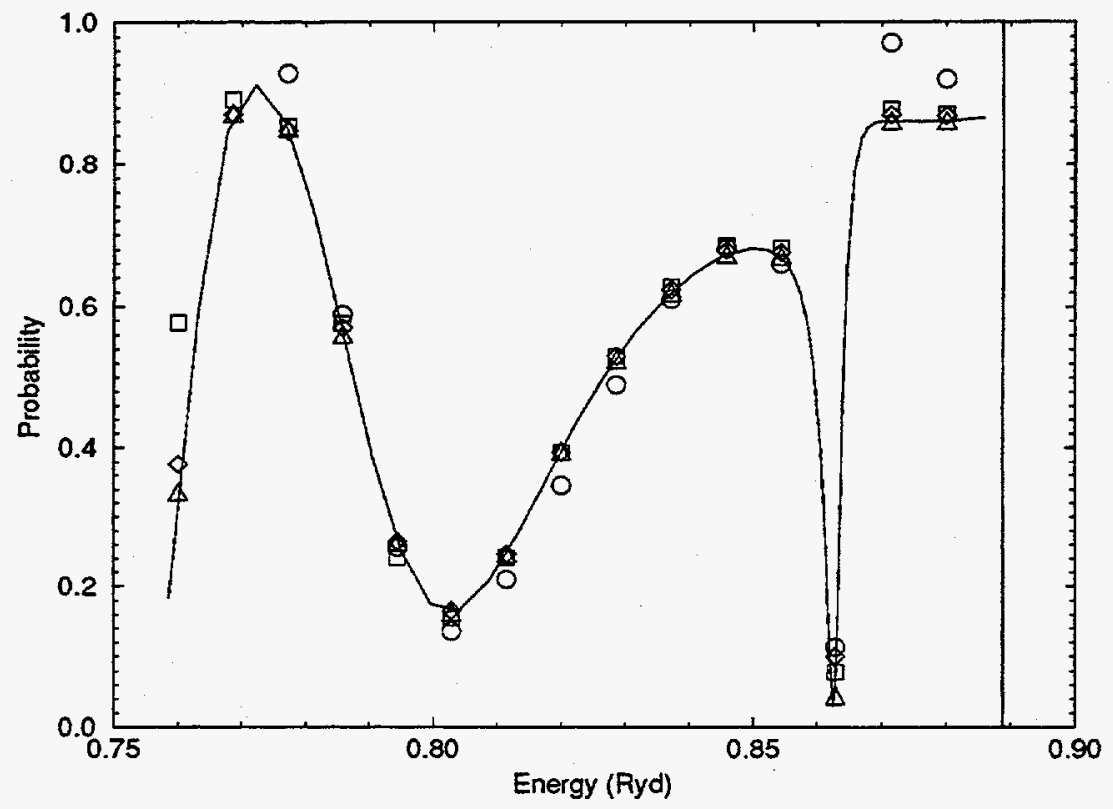

Figure 4.40: $n_{R}^{e}$ convergence of $\phi_{2 p} \rightarrow \phi_{2 p}$ transition probability $P_{33}$. Circle: $n_{R}^{e}=30$; Square: $n_{R}^{e}=35$; Diamond: $n_{R}^{e}=40$; Up triangle: $n_{R}^{e}=45$; Solid line: $n_{R}^{e}=50$. Vertical line is $n=3$ threshold. 
The convergence of the unitarity of the $\mathbf{S}$ matrix is first examined. Because of the manner in which the $\mathbf{S}$ matrix formulation of the Kohn variational principle imposes the asymptotic boundary conditions on the scattering wavefunction, the calculated $\mathbf{S}$ matrix is not necessarily unitary for a finite basis set. But the $\mathbf{S}$ matrix will approach unitarity as the basis set approaches completeness. As such, the unitarity of the $\mathbf{S}$ matrix provides yet another criterion for judging the accuracy of the calculation.

One of the most interesting parameters to vary in studying the convergence of the unitarity of the $\mathbf{S}$ matrix is the strength parameter $\alpha$. As was discovered previously, increasing values of $\alpha$ have the effect of placing more radial DVR points nearer to the origin. It would be expected that the density of radial DVR points would have the greatest effect on the accuracy of $\mathrm{S}$ matrix elements associated with the $\phi_{1 s}$ state (either as an incoming or outgoing channel) since this state has the highest percentage of its particle density close to the origin. This is in fact what is found. Examination of the diagonal elements of $\mathbf{S} \cdot \mathbf{S}^{\dagger}$ reveals that the $\left(\mathbf{S} \cdot \mathbf{S}^{\dagger}\right)_{11}$ element is the slowest to converge with respect to the $\alpha$ parameter. (The other two diagonal elements are actually quite converged for even modest values of $\alpha$.) Table 4.1 shows convergence of the unitarity of the real portion of $\left(\mathbf{S} \cdot \mathbf{S}^{\dagger}\right)_{11}$ for various values of the $\alpha$ parameter at several energies between the $n=2$ and $n=3$ thresholds (singlet). (The imaginary portion of the diagonal elements of $\mathbf{S} \cdot \mathbf{S}^{\dagger}$ is uniformly zero since $\mathbf{S}$ is complex symmetric.)

It is seen that the unitarity of the $\mathbf{S}$ matrix is weakest just above the $n=2$ threshold for all values of $\alpha$ studied. This is undoubtedly true because of the greater size of the interaction region in scattering calculations at very low asymptotic kinetic energies, and has little to do with the density of radial DVR points near the origin. At the higher kinetic energies close to the $n=3$ threshold it is found that larger values of $\alpha$ give better unitarity. There is also an unusual cyclic pattern demonstrated in the unitarity as the asymptotic kinetic energy is increased. This probably results from the fact that the actual distribution of radial DVR points provides a good representation of the scattering wavefunction for certain wavelengths in which peaks and troughs correspond to actual positions of the radial DVR points. This cyclic pattern can be 


\begin{tabular}{||c||c|c|c||}
\hline E (Ryd) & $\alpha=40$ & $\alpha=70$ & $\alpha=80$ \\
\hline 0.75400 & 4.94327 & 7.37066 & 9.66050 \\
0.75856 & 1.75667 & 1.11999 & 1.11524 \\
0.76310 & 1.04507 & 1.02939 & 1.00731 \\
0.76766 & 1.02964 & 1.02657 & 1.00379 \\
0.77220 & 1.02125 & 1.02510 & 1.00205 \\
0.77676 & 1.01464 & 1.02354 & 1.00104 \\
0.78132 & 1.00938 & 1.02170 & 1.00063 \\
0.78586 & 1.00545 & 1.01961 & 1.00080 \\
0.79042 & 1.00286 & 1.01730 & 1.00154 \\
0.79496 & 1.00166 & 1.01482 & 1.00278 \\
0.79952 & 1.00182 & 1.01227 & 1.00445 \\
0.80406 & 1.00332 & 1.00971 & 1.00643 \\
0.80862 & 1.00604 & 1.00728 & 1.00863 \\
0.81318 & 1.00983 & 1.00508 & 1.01096 \\
0.81772 & 1.01453 & 1.00322 & 1.01330 \\
0.82228 & 1.01991 & 1.00180 & 1.01554 \\
0.82682 & 1.02575 & 1.00090 & 1.01754 \\
0.83138 & 1.03181 & 1.00056 & 1.01921 \\
0.83594 & 1.03789 & 1.00082 & 1.02050 \\
0.84048 & 1.04380 & 1.00168 & 1.02140 \\
0.84504 & 1.04937 & 1.00311 & 1.02193 \\
0.84958 & 1.05451 & 1.00506 & 1.02215 \\
0.85414 & 1.05924 & 1.00748 & 1.02212 \\
0.85868 & 1.06445 & 1.01052 & 1.02217 \\
0.86324 & 1.07126 & 1.01739 & 1.02027 \\
0.86780 & 1.06426 & 1.01681 & 1.01834 \\
0.87234 & 1.06517 & 1.01954 & 1.01738 \\
0.87690 & 1.06516 & 1.02245 & 1.01608 \\
0.88144 & 1.06424 & 1.02522 & 1.01461 \\
0.88600 & 1.06252 & 1.02770 & 1.01306 \\
\hline
\end{tabular}

Table 4.1: Convergence of $\operatorname{Re}\left(\mathbf{S} \cdot \mathbf{S}^{\dagger}\right)_{11}$ with respect to $\alpha$ for $\alpha=40,70,80$ au. 
expected to vanish as the density of radial DVR points increases such that several points are available to represent a single wavelength.

Tables $4.2,4.3$, and 4.4 show convergence of the three $S_{1 x}$ matrix elements associated with the incoming $\phi_{1 s}$ state with respect to the positioning of the final radial DVR point at $R_{\max }$ for low, medium, and high kinetic energies between the $n=2$ and $n=3$ thresholds. (Convergence of the transition probabilities with respect to this parameter was shown in Figures 4.23 through 4.25 . Once again, convergence of the $\mathbf{S}$ matrix elements which do not involve the $\phi_{1 s}$ state is much more rapid and is not shown here.)

For the section of the energy spectrum closer to the $n=2$ threshold, the convergence of $S_{1 x}$ is obviously quite good. This is also true for the more intermediate kinetic energies. Convergence at the higher kinetic energies is more problematic, although the calculation at $R_{\max }=35$ au appears to achieve reasonable convergence for most of these energies also. But there is clearly much more difficulty entailed in converging $S_{1 x}$ near 0.86286 Ryd, where there is a Feshbach resonance (a narrow resonance due to the temporary capture of the free electron by an excited state of the hydrogen atom). This difficulty was also seen in the convergence analysis of the singlet transition probabilities in Figures 4.23 through 4.25.

One of the great benefits of this particular type of study (in which the $L^{2}$ basis is so localized) is that it provides an excellent mechanism for examining how large the interaction region must be in order to achieve a successful result. Conventional Slater-type basis sets utilize functions which are very delocalized and therefore cannot really provide this information in as pure a form. This series of calculations clearly shows that as the scattering energy approaches the $n=3$ threshold, the contributions of these closed channels becomes much more important. And while an interaction region $35 \mathrm{au}$ in length is adequate to represent the radial portions of the $n=3$ hydrogen states, the exponential tails of the scattering radial wavefunction at these higher kinetic energies are quite long and demand eventual inclusion of the closed $n=3$ channels in the reciprocal $R$ expansion composing the free functions. (It will be remembered that closed channels were not included in the reciprocal $R$ expansion because of the inability to assign non-zero coefficients to such terms in the 


\begin{tabular}{||c||r|r||r|r||r|r||}
\hline E (Ryd) & $\operatorname{Re} S_{11}$ & $\operatorname{Im} S_{11}$ & $\operatorname{Re} S_{12}$ & $\operatorname{Im} S_{12}$ & $\operatorname{Re} S_{13}$ & $\operatorname{Im} S_{13}$ \\
\hline 0.76000 & -0.02886 & 0.93092 & 0.28691 & -0.05804 & 0.03482 & 0.24548 \\
& -0.04154 & 0.92259 & 0.29621 & -0.05030 & 0.02521 & 0.25187 \\
& -0.06999 & 0.92848 & 0.30157 & -0.04003 & 0.00114 & 0.24974 \\
& -0.08429 & 0.92610 & 0.30533 & -0.03708 & -0.00072 & 0.25464 \\
& -0.07659 & 0.93575 & 0.30781 & -0.03744 & 0.00092 & 0.25934 \\
\hline 0.76858 & -0.02239 & 0.89314 & 0.22685 & -0.25436 & 0.21253 & 0.21053 \\
& -0.01673 & 0.89208 & 0.22677 & -0.25365 & 0.21252 & 0.21071 \\
& -0.03843 & 0.89739 & 0.22878 & -0.26134 & 0.20526 & 0.20892 \\
& -0.04797 & 0.89822 & 0.22771 & -0.25461 & 0.20846 & 0.21006 \\
& -0.04016 & 0.90523 & 0.22919 & -0.25272 & 0.20954 & 0.21191 \\
\hline 0.77714 & -0.01794 & 0.88623 & 0.09804 & -0.33183 & 0.28830 & 0.12345 \\
& -0.01521 & 0.88345 & 0.09748 & -0.33387 & 0.28985 & 0.12348 \\
& -0.03060 & 0.88353 & 0.10369 & -0.34411 & 0.28969 & 0.12509 \\
& -0.04052 & 0.88933 & 0.10477 & -0.33685 & 0.28771 & 0.12705 \\
& -0.03317 & 0.89405 & 0.10640 & -0.33534 & 0.28734 & 0.12897 \\
\hline 0.78572 & -0.01370 & 0.87623 & -0.01881 & -0.36441 & 0.31616 & 0.05295 \\
& -0.01234 & 0.87124 & -0.01759 & -0.36913 & 0.31933 & 0.05428 \\
& -0.02188 & 0.87273 & -0.01306 & -0.37241 & 0.32148 & 0.05737 \\
& -0.03332 & 0.88215 & -0.00907 & -0.36916 & 0.31531 & 0.05895 \\
& -0.02684 & 0.88341 & -0.00764 & -0.36883 & 0.31431 & 0.06056 \\
\hline 0.79428 & -0.00464 & 0.86640 & -0.11183 & -0.37750 & 0.31329 & -0.00206 \\
& -0.00423 & 0.86192 & -0.10938 & -0.38139 & 0.31656 & 0.00170 \\
& -0.01228 & 0.86422 & -0.11142 & -0.38077 & 0.31750 & 0.00384 \\
& -0.02558 & 0.87549 & -0.10517 & -0.38173 & 0.31111 & 0.00328 \\
& -0.01982 & 0.87297 & -0.10416 & -0.38158 & 0.31026 & 0.00398 \\
\hline
\end{tabular}

Table 4.2: $R_{\max }$ convergence of singlet $S_{1 x}$ matrix elements at lower kinetic energies for $R_{\max }=27,29,31,33$, and 35 au. 


\begin{tabular}{||c||c|c||c|c||c|c||}
\hline E (Ryd) & $\operatorname{Re} S_{11}$ & $\operatorname{Im} S_{11}$ & $\operatorname{Re} S_{12}$ & $\operatorname{Im} S_{12}$ & $\operatorname{Re} S_{13}$ & $\operatorname{Im} S_{13}$ \\
\hline 0.80286 & 0.00591 & 0.86113 & -0.18186 & -0.37724 & 0.29035 & -0.05043 \\
& 0.00313 & 0.85815 & -0.18109 & -0.37984 & 0.29145 & -0.04657 \\
& -0.00281 & 0.85814 & -0.18566 & -0.38017 & 0.29266 & -0.04701 \\
& -0.01756 & 0.86913 & -0.18027 & -0.38348 & 0.29035 & -0.04882 \\
& -0.01212 & 0.86305 & -0.17960 & -0.38294 & 0.29005 & -0.04858 \\
\hline 0.81142 & 0.01362 & 0.85810 & -0.23410 & -0.36640 & 0.26124 & -0.09950 \\
& 0.00743 & 0.85490 & -0.23543 & -0.36925 & 0.26213 & -0.09793 \\
& 0.00578 & 0.85344 & -0.23818 & -0.37067 & 0.26404 & -0.09788 \\
& -0.00893 & 0.86288 & -0.23628 & -0.37546 & 0.26623 & -0.09825 \\
& -0.00381 & 0.85443 & -0.23530 & -0.37455 & 0.26567 & -0.09793 \\
\hline 0.82000 & 0.01975 & 0.85372 & -0.27482 & -0.34868 & 0.23702 & -0.14506 \\
& 0.01260 & 0.85006 & -0.27637 & -0.35291 & 0.23906 & -0.14440 \\
& 0.01393 & 0.84949 & -0.27601 & -0.35338 & 0.23965 & -0.14333 \\
& 0.00057 & 0.85742 & -0.27754 & -0.35925 & 0.24384 & -0.14163 \\
& 0.00417 & 0.84799 & -0.27588 & -0.35823 & 0.24257 & -0.14169 \\
\hline 0.82858 & 0.02748 & 0.84852 & -0.30597 & -0.32882 & 0.21858 & -0.18172 \\
& 0.02080 & 0.84656 & -0.30506 & -0.33318 & 0.22092 & -0.18071 \\
& 0.02196 & 0.84655 & -0.30417 & -0.33162 & 0.22006 & -0.17977 \\
& 0.01061 & 0.85329 & -0.30746 & -0.33782 & 0.22430 & -0.17714 \\
& 0.01109 & 0.84390 & -0.30549 & -0.33726 & 0.22295 & -0.17786 \\
\hline 0.83714 & 0.03753 & 0.84550 & -0.32707 & -0.30785 & 0.20450 & -0.20762 \\
& 0.03029 & 0.84685 & -0.32353 & -0.31003 & 0.20599 & -0.20610 \\
& 0.03009 & 0.84520 & -0.32447 & -0.30864 & 0.20484 & -0.20600 \\
& 0.02130 & 0.85095 & -0.32759 & -0.31443 & 0.20855 & -0.20341 \\
& 0.01708 & 0.84275 & -0.32598 & -0.31489 & 0.20790 & -0.20455 \\
\hline
\end{tabular}

Table 4.3: $R_{\max }$ convergence of singlet $S_{1 x}$ matrix elements at medium kinetic energies for $R_{\max }=27,29,31,33$, and 35 au. 


\begin{tabular}{||c||c|c||r|r||r|r||}
\hline E (Ryd) & $\operatorname{Re} S_{11}$ & $\operatorname{Im} S_{11}$ & $\operatorname{Re} S_{12}$ & $\operatorname{Im} S_{12}$ & $\operatorname{Re} S_{13}$ & $\operatorname{Im} S_{13}$ \\
\hline 0.84572 & 0.04840 & 0.84696 & -0.33851 & -0.28574 & 0.19419 & -0.22132 \\
& 0.03955 & 0.85107 & -0.33359 & -0.28586 & 0.19484 & -0.21950 \\
& 0.03897 & 0.84722 & -0.33512 & -0.28717 & 0.19520 & -0.21950 \\
& 0.03321 & 0.85217 & -0.33646 & -0.29205 & 0.19852 & -0.21690 \\
& 0.02289 & 0.84642 & -0.33592 & -0.29391 & 0.19917 & -0.21801 \\
\hline 0.85428 & 0.05933 & 0.85560 & -0.33695 & -0.26477 & 0.18988 & -0.21685 \\
& 0.05002 & 0.86288 & -0.32747 & -0.26548 & 0.19262 & -0.21072 \\
& 0.04996 & 0.86023 & -0.32358 & -0.26975 & 0.19676 & -0.20508 \\
& 0.04782 & 0.86565 & -0.31986 & -0.27383 & 0.20090 & -0.19899 \\
& 0.03082 & 0.86401 & -0.31977 & -0.27750 & 0.20352 & -0.19858 \\
\hline 0.86286 & 0.06804 & 0.89107 & -0.28828 & -0.24378 & 0.19708 & -0.14807 \\
& 0.04761 & 0.93294 & -0.21705 & -0.22205 & 0.18822 & -0.05803 \\
& -0.00749 & 0.97749 & -0.13345 & -0.12329 & 0.08591 & 0.07590 \\
& -0.09784 & 0.97151 & -0.15781 & 0.04234 & -0.12852 & 0.09003 \\
& -0.15010 & 0.95157 & -0.20484 & 0.08374 & -0.19492 & 0.04638 \\
\hline 0.87142 & -0.12012 & 0.80776 & -0.42620 & 0.10954 & -0.29242 & -0.23519 \\
& -0.00458 & 0.78117 & -0.49054 & -0.10141 & -0.03117 & -0.38102 \\
& 0.01460 & 0.79100 & -0.46966 & -0.14987 & 0.03674 & -0.36327 \\
& 0.02570 & 0.79871 & -0.45761 & -0.16661 & 0.06173 & -0.35050 \\
& -0.00118 & 0.80676 & -0.45854 & -0.17680 & 0.07219 & -0.34836 \\
\hline 0.88000 & 0.03772 & 0.78002 & -0.48492 & -0.11950 & 0.01137 & -0.37669 \\
& 0.04651 & 0.80517 & -0.45452 & -0.15487 & 0.06255 & -0.35067 \\
& 0.04309 & 0.81037 & -0.44228 & -0.17046 & 0.08425 & -0.33574 \\
& 0.05098 & 0.81455 & -0.43360 & -0.17886 & 0.09634 & -0.32486 \\
& 0.01987 & 0.82834 & -0.43412 & -0.18772 & 0.10496 & -0.32111 \\
\hline
\end{tabular}

Table 4.4: $R_{\max }$ convergence of singlet $S_{1 x}$ matrix elements at higher kinetic energies for $R_{\text {max }}=27,29,31,33$, and 35 au. 
asymptotic region.) It is fairly clear that completeness within the spatial confines of the interaction region should not be the troubling factor, as the kinetic energies of the radial scattering wavefunction are well represented by the grid spacing employed, as demonstrated in Figures 4.7 through 4.10 .

\subsubsection{Transition Probabilities and S Matrices}

Tables 4.5 through 4.13 show values of calculated $S$ matrices for singlet scattering using the convergence parameters previously reported. The tables show values for both the real and imaginary parts of the $\mathbf{S}$ matrix for a wide range of energies between the $n=2$ and $n=3$ thresholds, as well as individual transition probabilities. (Only values of $S_{11}, S_{12}, S_{13}, S_{22}, S_{23}$, and $S_{33}$ are shown because of the inherent symmetry of the $\mathbf{S}$ matrix.) Calculations were performed in a series of sequences each using equally spaced values of total energy. In areas where Feshbach resonances were previously reported to have been found by Callaway [97] a finer energy grid was employed to attempt to duplicate these results. The spacing of the energy grid in these regions was based upon the widths of the resonances reported by Callaway.

Callaway reports resonances just below the $n=3$ threshold at 0.86199 , $0.88445,0.88773$, and 0.88799 Ryd for $J=0$ and $S=0$. Only the first (and widest) resonance at 0.86199 Ryd is seen in the current DVR calculations at 0.86200 Ryd. The other three resonances are located very close to the $n=3$ threshold and it is suspected that the current calculation would encounter difficulty in this energy region because of the lack of extremely long range $L^{2}$ functions in the basis set needed to accurately represent the long exponential tails present in the scattering wavefunction when the scattering energy is just beneath a threshold. On the other hand, more recent calculations by Wang and Callaway [69] which employ a direct numerical solution (Numerov algorithm) for the same problem report only the first resonance and not the other three.

Figures 4.41 through 4.43 show the final calculated values of the transition probabilities for singlet scattering from each of the $\phi_{1 s}, \phi_{2 s}$, and $\phi_{2 p}$ states for energies 


\begin{tabular}{||c||r|r|r|r|r|r||}
\hline E (Ryd) & $\operatorname{Re} S_{11}$ & $\operatorname{Re} S_{12}$ & $\operatorname{Re} S_{13}$ & $\operatorname{Re} S_{22}$ & \multicolumn{1}{c}{$\operatorname{Re} S_{23}$} & \multicolumn{1}{c}{$\operatorname{Re} S_{33}$} \\
& $\operatorname{Im} S_{11}$ & $\operatorname{Im} S_{12}$ & $\operatorname{Im} S_{13}$ & $\operatorname{Im} S_{22}$ & $\operatorname{Im} S_{23}$ & \multicolumn{1}{|c}{$S_{33}$} \\
& $\left|S_{11}\right|^{2}$ & $\left|S_{12}\right|^{2}$ & $\left|S_{13}\right|^{2}$ & $\left|S_{22}\right|^{2}$ & $\left|S_{23}\right|^{2}$ & $\left|S_{33}\right|^{2}$ \\
\hline 0.75856 & -0.15287 & 0.25947 & -0.11315 & 0.25310 & -0.86210 & -0.43159 \\
& 0.97906 & 0.06473 & 0.22135 & 0.30388 & -0.40211 & -0.09755 \\
& 0.98193 & 0.07151 & 0.06180 & 0.15640 & 0.90491 & 0.19579 \\
\hline 0.76310 & -0.05474 & 0.30296 & 0.10541 & 0.73666 & -0.58857 & -0.76255 \\
& 0.90127 & -0.14155 & 0.26285 & 0.03303 & -0.03458 & 0.10476 \\
& 0.81528 & 0.11182 & 0.08020 & 0.54376 & 0.34761 & 0.59246 \\
\hline 0.76766 & -0.05068 & 0.24524 & 0.19406 & 0.85083 & -0.29771 & -0.86705 \\
& 0.89336 & -0.23579 & 0.22303 & -0.27393 & 0.03683 & 0.28975 \\
& 0.80066 & 0.11574 & 0.08740 & 0.79895 & 0.08999 & 0.83573 \\
\hline 0.77220 & -0.05087 & 0.17936 & 0.25100 & 0.79581 & -0.04851 & -0.86475 \\
& 0.88584 & -0.29629 & 0.17829 & -0.50026 & -0.04028 & 0.40158 \\
& 0.78730 & 0.11996 & 0.09479 & 0.88357 & 0.00398 & 0.90906 \\
\hline 0.77676 & -0.05031 & 0.11525 & 0.28715 & 0.67267 & 0.13805 & -0.82188 \\
& 0.87844 & -0.33517 & 0.13698 & -0.61773 & -0.17240 & 0.42238 \\
& 0.77419 & 0.12562 & 0.10122 & 0.83408 & 0.04878 & 0.85389 \\
\hline 0.78132 & -0.04890 & 0.05507 & 0.30778 & 0.52914 & 0.27263 & -0.76445 \\
& 0.87293 & -0.35841 & 0.10008 & -0.65354 & -0.30670 & 0.38042 \\
& 0.76440 & 0.13149 & 0.10474 & 0.70710 & 0.16839 & 0.72910 \\
\hline 0.79496 & -0.05001 & -0.10552 & 0.31002 & 0.07687 & 0.56866 & -0.49522 \\
& 0.86395 & -0.38287 & 0.00663 & -0.42288 & -0.58033 & -0.04367 \\
& 0.74891 & 0.15772 & 0.09616 & 0.18474 & 0.66016 & 0.24715 \\
\hline 0.78586 & -0.04817 & -0.00206 & 0.31670 & 0.38083 & 0.38121 & -0.69800 \\
& 0.86955 & -0.37091 & 0.06689 & -0.62685 & -0.42856 & 0.28550 \\
& 0.75844 & 0.13758 & 0.10477 & 0.53797 & 0.32898 & 0.56871 \\
\hline 0.79042 & -0.04882 & -0.05626 & 0.31659 & 0.22956 & 0.48236 & -0.61195 \\
& 0.86697 & -0.37789 & 0.03628 & -0.54344 & -0.52562 & 0.13540 \\
& 0.75402 & 0.14597 & 0.10155 & 0.34802 & 0.50895 & 0.39282 \\
\hline 0.79952 & -0.05035 & -0.14778 & 0.30022 & -0.06664 & 0.62292 & -0.36039 \\
& 0.86033 & -0.38634 & -0.02264 & -0.29718 & -0.59227 & -0.20827 \\
& 0.74270 & 0.17110 & 0.09064 & 0.09276 & 0.73881 & 0.17326 \\
\hline 0
\end{tabular}

Table 4.5: Converged $\mathbf{S}$ matrix elements for singlet scattering with $\alpha=80, n_{\gamma}=4$, $R_{\max }=35$ au, $p_{\max }=8$, and $n_{R}^{e}=50$. 


\begin{tabular}{|c|c|c|c|c|c|c|}
\hline$E(\mathrm{Ryd})$ & $\begin{array}{c}\operatorname{Re} S_{11} \\
\operatorname{Im} S_{11} \\
\left|S_{11}\right|^{2}\end{array}$ & $\begin{array}{r}\operatorname{Re} S_{12} \\
\operatorname{Im} S_{12} \\
\left|S_{12}\right|^{2}\end{array}$ & $\begin{array}{c}\text { Re } S_{13} \\
\operatorname{Im} S_{13} \\
\left|S_{13}\right|^{2}\end{array}$ & $\begin{array}{r}\operatorname{Re} S_{22} \\
\operatorname{Im} S_{22} \\
\left|S_{22}\right|^{2}\end{array}$ & $\begin{array}{r}\operatorname{Re} S_{23} \\
\operatorname{Im} S_{23} \\
\left|S_{23}\right|^{2}\end{array}$ & $\begin{array}{r}\operatorname{Re} S_{33} \\
\operatorname{Im} S_{33} \\
\left|S_{33}\right|^{2}\end{array}$ \\
\hline \multirow[t]{3}{*}{0.80406} & -0.04890 & -0.18268 & 0.28915 & -0.19120 & 0.64592 & -0.22769 \\
\hline & 0.85698 & -0.38743 & -0.05047 & -0.18542 & -0.57520 & -0.34006 \\
\hline & 0.73681 & 0.18347 & 0.08615 & 0.07094 & 0.74807 & 0.16748 \\
\hline \multirow[t]{3}{*}{0.80862} & -0.04581 & -0.21100 & 0.27703 & -0.29858 & 0.64898 & -0.10087 \\
\hline & 0.85496 & -0.38535 & -0.07623 & -0.09011 & -0.53911 & -0.44312 \\
\hline & 0.73306 & 0.19302 & 0.08256 & 0.09727 & 0.71181 & 0.20653 \\
\hline \multirow[t]{3}{*}{0.81318} & -0.04200 & -0.23410 & 0.26401 & -0.39399 & 0.63742 & 0.02228 \\
\hline & 0.85468 & -0.37966 & -0.10038 & -0.00797 & -0.49111 & -0.52222 \\
\hline & 0.73224 & 0.19894 & 0.07978 & 0.15529 & 0.64749 & 0.27321 \\
\hline \multirow[t]{3}{*}{0.81772} & -0.03835 & -0.25371 & 0.25096 & -0.47681 & 0.61158 & 0.13538 \\
\hline & 0.85554 & -0.37053 & -0.12342 & 0.06445 & -0.44074 & -0.57932 \\
\hline & 0.73342 & 0.20166 & 0.07821 & 0.23150 & 0.56828 & 0.35394 \\
\hline \multirow[t]{3}{*}{0.82228} & -0.03498 & -0.27131 & 0.23888 & -0.54255 & 0.57464 & 0.22765 \\
\hline & 0.85657 & -0.35897 & -0.14516 & 0.12994 & -0.39675 & -0.61888 \\
\hline & 0.73494 & 0.20247 & 0.07814 & 0.31124 & 0.48762 & 0.43484 \\
\hline \multirow[t]{3}{*}{0.82682} & -0.03137 & -0.28751 & 0.22833 & -0.58940 & 0.53345 & 0.29538 \\
\hline & 0.85703 & -0.34645 & -0.16502 & 0.18954 & -0.36187 & -0.64699 \\
\hline & 0.73548 & 0.20269 & 0.07937 & 0.38332 & 0.41552 & 0.50585 \\
\hline \multirow[t]{3}{*}{0.83138} & -0.02678 & -0.30200 & 0.21950 & -0.62039 & 0.49450 & 0.34412 \\
\hline & 0.85684 & -0.33416 & -0.18238 & 0.24205 & -0.33361 & -0.66714 \\
\hline & 0.73489 & 0.20287 & 0.08144 & 0.44347 & 0.35583 & 0.56349 \\
\hline \multirow[t]{3}{*}{0.83594} & -0.02065 & -0.31396 & 0.21244 & -0.64088 & 0.46163 & 0.38320 \\
\hline & 0.85651 & -0.32265 & -0.19662 & 0.28559 & -0.30860 & -0.67958 \\
\hline & 0.73404 & 0.20267 & 0.08379 & 0.49229 & 0.30834 & 0.60867 \\
\hline \multirow[t]{3}{*}{0.84048} & -0.01290 & -0.32250 & 0.20715 & -0.65541 & 0.43636 & 0.42008 \\
\hline & 0.85687 & -0.31185 & -0.20697 & 0.31932 & -0.28546 & -0.68327 \\
\hline & 0.73439 & 0.20126 & 0.08575 & 0.53153 & 0.27190 & 0.64333 \\
\hline \multirow[t]{3}{*}{0.84504} & -0.00383 & -0.32667 & 0.20372 & -0.66612 & 0.41981 & 0.45835 \\
\hline & 0.85895 & -0.30142 & -0.21225 & 0.34333 & -0.26537 & -0.67677 \\
\hline & 0.73781 & 0.19757 & $0: 08655$ & 0.56159 & 0.24666 & 0.66810 \\
\hline
\end{tabular}

Table 4.6: Converged $S$ matrix elements for singlet scattering with $\alpha=80, n_{\gamma}=4$, $R_{\max }=35$ au, $p_{\max }=8$, and $n_{R}^{e}=50$. 


\begin{tabular}{|c|c|c|c|c|c|c|}
\hline$E(R y d)$ & $\begin{array}{l}\operatorname{Re} S_{11} \\
\operatorname{Im} S_{11} \\
\left|S_{11}\right|^{2}\end{array}$ & $\begin{array}{r}\operatorname{Re} S_{12} \\
\operatorname{Im} S_{12} \\
\left|S_{12}\right|^{2}\end{array}$ & $\begin{array}{l}\operatorname{Re} S_{13} \\
\operatorname{Im} S_{13} \\
\left|S_{13}\right|^{2}\end{array}$ & $\begin{array}{r}\operatorname{Re} S_{22} \\
\operatorname{Im} S_{22} \\
\left|S_{22}\right|^{2}\end{array}$ & $\begin{array}{r}\operatorname{Re} S_{23} \\
\operatorname{Im} S_{23} \\
\left|S_{23}\right|^{2}\end{array}$ & $\begin{array}{r}\operatorname{Re} S_{33} \\
\operatorname{Im} S_{33} \\
\left|S_{33}\right|^{2}\end{array}$ \\
\hline \multirow[t]{3}{*}{0.84958} & 0.00612 & -0.32469 & 0.20270 & -0.67310 & 0.41592 & 0.49982 \\
\hline & 0.86412 & -0.29126 & -0.20992 & 0.35606 & -0.25121 & -0.65622 \\
\hline & 0.74674 & 0.19026 & 0.08515 & 0.57984 & 0.23610 & 0.68044 \\
\hline \multirow[t]{3}{*}{0.85200} & 0.01165 & -0.31942 & 0.20367 & -0.67492 & 0.42318 & 0.52461 \\
\hline & 0.86910 & -0.28604 & -0.20335 & 0.35543 & -0.24739 & -0.63520 \\
\hline & 0.75547 & 0.18385 & 0.08283 & 0.58185 & 0.24028 & 0.67869 \\
\hline \multirow[t]{3}{*}{0.85306} & 0.01409 & -0.31557 & 0.20456 & -0.67513 & 0.42990 & 0.53658 \\
\hline & 0.87211 & -0.28377 & -0.19851 & 0.35241 & -0.24670 & -0.62208 \\
\hline & 0.76077 & 0.18011 & 0.08125 & 0.57999 & 0.24567 & 0.67490 \\
\hline \multirow[t]{3}{*}{0.85410} & 0.01654 & -0.31034 & 0.20579 & -0.67481 & 0.43985 & 0.54957 \\
\hline & 0.87587 & -0.28144 & -0.19191 & 0.34690 & -0.24663 & -0.60525 \\
\hline & 0.76742 & 0.17552 & 0.07918 & 0.57571 & 0.25429 & 0.66835 \\
\hline \multirow[t]{3}{*}{0.85414} & 0.01662 & -0.31015 & 0.20583 & -0.67479 & 0.44022 & 0.55000 \\
\hline & 0.87600 & -0.28137 & -0.19167 & 0.34668 & -0.24663 & -0.60466 \\
\hline & 0.76765 & 0.17536 & 0.07910 & 0.57553 & 0.25462 & 0.66811 \\
\hline \multirow[t]{3}{*}{0.85516} & 0.01894 & -0.30318 & 0.20735 & -0.67367 & 0.45430 & 0.56384 \\
\hline & 0.88067 & -0.27894 & -0.18285 & 0.33794 & -0.24706 & -0.58313 \\
\hline & 0.77594 & 0.16973 & 0.07643 & 0.56803 & 0.26743 & 0.65796 \\
\hline \multirow[t]{3}{*}{0.85622} & 0.02117 & -0.29327 & 0.20914 & -0.67110 & 0.47529 & 0.57955 \\
\hline & 0.88699 & -0.27600 & -0.17019 & 0.32404 & -0.24766 & -0.55311 \\
\hline & 0.78720 & 0.16218 & 0.07270 & 0.55538 & 0.28724 & 0.64181 \\
\hline \multirow[t]{3}{*}{0.85726} & 0.02293 & -0.27922 & 0.21077 & -0.66577 & 0.50624 & 0.59651 \\
\hline & 0.89560 & -0.27197 & -0.15206 & 0.30274 & -0.24745 & -0.51069 \\
\hline & 0.80263 & 0.15193 & 0.06755 & 0.53490 & 0.31751 & 0.61663 \\
\hline \multirow[t]{3}{*}{0.85832} & 0.02343 & -0.25867 & 0.21093 & -0.65449 & 0.55312 & 0.61299 \\
\hline & 0.90784 & -0.26527 & -0.12506 & 0.26994 & -0.24362 & -0.44765 \\
\hline & 0.82472 & 0.13728 & 0.06013 & 0.50122 & 0.36529 & 0.57615 \\
\hline \multirow[t]{3}{*}{0.85868} & 0.02297 & -0.24911 & 0.21002 & -0.64777 & 0.57544 & 0.61771 \\
\hline & 0.91348 & -0.26159 & -0.11229 & 0.25434 & -0.24013 & -0.41766 \\
\hline & 0.83497 & 0.13049 & 0.05672 & 0.48429 & 0.38879 & 0.55601 \\
\hline
\end{tabular}

Table 4.7: Converged $\mathbf{S}$ matrix elements for singlet scattering with $\alpha=80, n_{\gamma}=4$, $R_{\max }=35$ au, $p_{\max }=8$, and $n_{R}^{e}=50$. 


\begin{tabular}{||c||r|r|r|r|r|r||}
\hline E (Ryd) & $\operatorname{Re} S_{11}$ & $\operatorname{Re} S_{12}$ & $\operatorname{Re} S_{13}$ & $\operatorname{Re} S_{22}$ & $\operatorname{Re} S_{23}$ & $\operatorname{Re} S_{33}$ \\
& $\operatorname{Im} S_{11}$ & $\operatorname{Im} S_{12}$ & $\operatorname{Im} S_{13}$ & $\operatorname{Im} S_{22}$ & $\operatorname{Im} S_{23}$ & $\operatorname{Im} S_{33}$ \\
& $\left|S_{11}\right|^{2}$ & $\left|S_{12}\right|^{2}$ & $\left|S_{13}\right|^{2}$ & $\left|S_{22}\right|^{2}$ & $\left|S_{23}\right|^{2}$ & $\left|S_{33}\right|^{2}$ \\
\hline 0.85936 & 0.02046 & -0.22758 & 0.20542 & -0.62888 & 0.62675 & 0.62165 \\
& 0.92611 & -0.25156 & -0.08295 & 0.21886 & -0.22769 & -0.34815 \\
& 0.85810 & 0.11508 & 0.04908 & 0.44339 & 0.44466 & 0.50766 \\
\hline 0.86040 & 0.00753 & -0.18159 & 0.18057 & -0.56653 & 0.74156 & 0.59436 \\
& 0.95323 & -0.21883 & -0.01686 & 0.14299 & -0.17368 & -0.18691 \\
& 0.90870 & 0.08086 & 0.03289 & 0.34140 & 0.58008 & 0.38820 \\
\hline 0.86042 & 0.00706 & -0.18047 & 0.17963 & -0.56452 & 0.74446 & 0.59294 \\
& 0.95390 & -0.21778 & -0.01518 & 0.14117 & -0.17175 & -0.18271 \\
& 0.90998 & 0.08000 & 0.03250 & 0.33861 & 0.58372 & 0.38496 \\
\hline 0.86080 & -0.00371 & -0.15956 & 0.15767 & -0.52055 & 0.79996 & 0.55650 \\
& 0.96649 & -0.19447 & 0.01733 & 0.10742 & -0.12758 & -0.10001 \\
& 0.93412 & 0.06328 & 0.02516 & 0.28251 & 0.65621 & 0.31969 \\
\hline 0.86120 & -0.02140 & -0.13761 & 0.12022 & -0.45324 & 0.86247 & 0.48648 \\
& 0.98015 & -0.15839 & 0.05481 & 0.07347 & -0.05462 & -0.00030 \\
& 0.96115 & 0.04402 & 0.01746 & 0.21082 & 0.74684 & 0.23666 \\
\hline 0.86148 & -0.03857 & -0.12540 & 0.08257 & -0.39110 & 0.90212 & 0.41075 \\
& 0.98831 & -0.12465 & 0.07958 & 0.05651 & 0.01700 & 0.07028 \\
& 0.97824 & 0.03126 & 0.01315 & 0.15615 & 0.81411 & 0.17365 \\
\hline 0.86160 & -0.04811 & -0.12154 & 0.06117 & -0.35749 & 0.91739 & 0.36607 \\
& 0.99121 & -0.10624 & 0.08962 & 0.05226 & 0.05720 & 0.10116 \\
& 0.98481 & 0.02606 & 0.01177 & 0.13053 & 0.84488 & 0.14424 \\
\hline 0.86200 & -0.08502 & -0.12312 & -0.02445 & -0.23243 & 0.93826 & 0.17867 \\
& 0.99327 & -0.03674 & 0.10757 & 0.06533 & 0.21516 & 0.17369 \\
& 0.99381 & 0.01651 & 0.01217 & 0.05829 & 0.92663 & 0.06209 \\
\hline 0.86240 & -0.12732 & -0.15813 & -0.12898 & -0.09802 & 0.88539 & -0.06629 \\
& 0.97730 & 0.04025 & 0.08626 & 0.14009 & 0.40242 & 0.16328 \\
& 0.97133 & 0.02663 & 0.02408 & 0.02923 & 0.94586 & 0.03105 \\
\hline 0.86252 & -0.13972 & -0.17768 & -0.16153 & -0.06092 & 0.84842 & -0.14668 \\
& 0.96751 & 0.06222 & 0.06834 & 0.17882 & 0.45928 & 0.13421 \\
& 0.95560 & 0.03544 & 0.03076 & 0.03569 & 0.93075 & 0.03953 \\
\hline & & & & & & \\
\hline
\end{tabular}

Table 4.8: Converged $S$ matrix elements for singlet scattering with $\alpha=80, n_{\gamma}=4$, $R_{\max }=35$ au, $p_{\max }=8$, and $n_{R}^{e}=50$. 


\begin{tabular}{|c|c|c|c|c|c|c|}
\hline $\mathrm{E}(\mathrm{Ryd})$ & $\begin{array}{r}\operatorname{Re} S_{11} \\
\operatorname{Im} S_{11} \\
\left|S_{11}\right|^{2}\end{array}$ & $\begin{array}{c}\operatorname{Re} S_{12} \\
\operatorname{Im} S_{12} \\
\left|S_{12}\right|^{2}\end{array}$ & $\begin{array}{l}\operatorname{Re} S_{13} \\
\operatorname{Im} S_{13} \\
\left|S_{13}\right|^{2}\end{array}$ & $\begin{array}{r}\operatorname{Re} S_{22} \\
\operatorname{Im} S_{22} \\
\left|S_{22}\right|^{2}\end{array}$ & $\begin{array}{r}\operatorname{Re} S_{23} \\
\operatorname{Im} S_{23} \\
\left|S_{23}\right|^{2}\end{array}$ & $\begin{array}{c}\operatorname{Re} S_{33} \\
\operatorname{Im} S_{33} \\
\left|S_{33}\right|^{2}\end{array}$ \\
\hline \multirow[t]{3}{*}{0.86280} & -0.16102 & -0.23190 & -0.22181 & -0.00207 & 0.73716 & -0.30394 \\
\hline & 0.93939 & 0.09879 & 0.01163 & 0.28288 & 0.56158 & 0.02663 \\
\hline & 0.90838 & 0.06354 & 0.04933 & 0.08003 & 0.85878 & 0.09309 \\
\hline \multirow[t]{3}{*}{0.86320} & -0.17207 & -0.32351 & -0.26696 & 0.01416 & 0.53263 & -0.44506 \\
\hline & 0.89005 & 0.11469 & -0.09735 & 0.45260 & 0.62972 & -0.20598 \\
\hline & 0.82180 & 0.11781 & 0.08074 & 0.20505 & 0.68024 & 0.24051 \\
\hline \multirow[t]{3}{*}{0.86324} & -0.17179 & -0.33264 & -0.26834 & 0.01121 & 0.51132 & -0.45226 \\
\hline & 0.88504 & 0.11376 & -0.10894 & 0.46920 & 0.63075 & -0.23196 \\
\hline & 0.81281 & 0.12359 & 0.08387 & 0.22027 & 0.65929 & 0.25834 \\
\hline \multirow[t]{3}{*}{0.86358} & -0.16183 & -0.39813 & -0.26046 & -0.03601 & 0.35325 & -0.46446 \\
\hline & 0.84855 & 0.09281 & -0.19615 & 0.58654 & 0.60531 & -0.43413 \\
\hline & 0.74623 & 0.16712 & 0.10631 & 0.34533 & 0.49119 & 0.40419 \\
\hline \multirow[t]{3}{*}{0.86360} & -0.16086 & -0.40158 & -0.25905 & -0.03996 & 0.34463 & -0.46288 \\
\hline & 0.84660 & 0.09090 & -0.20097 & 0.59262 & 0.60213 & -0.44567 \\
\hline & 0.74261 & 0.16953 & 0.10750 & 0.35280 & 0.48133 & 0.41288 \\
\hline \multirow[t]{3}{*}{0.86400} & -0.13856 & -0.45234 & -0.22057 & -0.12388 & 0.21245 & -0.40040 \\
\hline & 0.81739 & 0.04878 & -0.27603 & 0.68066 & 0.52265 & -0.63115 \\
\hline & 0.68733 & 0.20699 & 0.12484 & 0.47864 & 0.31830 & 0.55867 \\
\hline \multirow[t]{3}{*}{0.86440} & -0.11470 & -0.47957 & -0.17383 & -0.20697 & 0.13365 & -0.30857 \\
\hline & 0.80101 & 0.00544 & -0.32230 & 0.72579 & 0.43185 & -0.75379 \\
\hline & 0.65477 & 0.23002 & 0.13409 & 0.56961 & 0.20436 & 0.66341 \\
\hline \multirow[t]{3}{*}{0.86464} & -0.10200 & -0.48795 & -0.14782 & -0.24947 & 0.10587 & -0.25421 \\
\hline & 0.79567 & -0.01709 & -0.33920 & 0.73881 & 0.38245 & -0.80178 \\
\hline & 0.64349 & 0.23839 & 0.13691 & 0.60808 & 0.15748 & 0.70747 \\
\hline \multirow[t]{3}{*}{0.86568} & -0.05917 & -0.49420 & -0.05683 & -0.38515 & 0.06321 & -0.05209 \\
\hline & 0.78996 & -0.09015 & -0.36806 & 0.74380 & 0.21353 & -0.90042 \\
\hline & 0.62754 & 0.25236 & 0.13870 & 0.70158 & 0.04959 & 0.81347 \\
\hline \multirow[t]{3}{*}{0.86674} & -0.03436 & -0.48452 & -0.00335 & -0.45702 & 0.06943 & 0.07482 \\
\hline & 0.79424 & -0.12927 & -0.36742 & 0.72408 & 0.11655 & -0.91793 \\
\hline & 0.63200 & 0.25147 & 0.13501 & 0.73316 & 0.01840 & 0.84819 \\
\hline
\end{tabular}

Table 4.9: Converged $S$ matrix elements for singlet scattering with $\alpha=80, n_{\gamma}=4$, $R_{\max }=35$ au, $p_{\max }=8$, and $n_{R}^{e}=50$. 


\begin{tabular}{|c|c|c|c|c|c|c|}
\hline E (Ryd) & $\begin{array}{l}\operatorname{Re} S_{11} \\
\operatorname{Im} S_{11} \\
\left|S_{11}\right|^{2}\end{array}$ & $\begin{array}{r}\operatorname{Re} S_{12} \\
\operatorname{Im} S_{12} \\
\left|S_{12}\right|^{2}\end{array}$ & $\begin{array}{r}\operatorname{Re} S_{13} \\
\operatorname{Im} S_{13} \\
\left|S_{13}\right|^{2}\end{array}$ & $\begin{array}{c}\operatorname{Re} S_{22} \\
\operatorname{Im} S_{22} \\
\left|S_{22}\right|^{2}\end{array}$ & $\begin{array}{r}\operatorname{Re} S_{23} \\
\operatorname{Im} S_{23} \\
\left|S_{23}\right|^{2}\end{array}$ & $\begin{array}{r}\operatorname{Re} S_{33} \\
\operatorname{Im} S_{33} \\
\left|S_{33}\right|^{2}\end{array}$ \\
\hline \multirow[t]{3}{*}{0.86778} & -0.01898 & -0.47409 & 0.02898 & -0.49744 & 0.08374 & 0.15537 \\
\hline & 0.79957 & -0.15118 & -0.36085 & 0.70502 & 0.05878 & -0.91422 \\
\hline & 0.63967 & .24762 & .13105 & 0.74450 & .01047 & 0.85994 \\
\hline \multirow[t]{3}{*}{0.86780} & -0.01894 & 5.47405 & 0.02906 & -0.49754 & 0.08379 & 0.15559 \\
\hline & 0.79959 & -0.15124 & -0.36083 & 0.70497 & 0.05863 & -0.91420 \\
\hline & 0.63970 & 0.24760 & 0.13104 & 0.74453 & 0.01046 & 0.85997 \\
\hline \multirow[t]{3}{*}{0.86884} & -0.00857 & -0.46539 & 0.04978 & -0.52195 & 0.09738 & 0.20965 \\
\hline & 0.80426 & -0.16425 & -0.35386 & 0.69010 & 0.02203 & -0.90539 \\
\hline & 0.64691 & 0.24357 & 0.12769 & 0.74867 & 0.00997 & 0.86368 \\
\hline \multirow[t]{3}{*}{0.86990} & -0.00094 & -0.45848 & 0.06395 & -0.53777 & 0.10877 & 0.24841 \\
\hline & 0.80812 & -0.17244 & -0.347666 & 0.67890 & -0.00274 & -0.895955 \\
\hline & & 0.23994 & 0.12496 & 0.75010 & 0.01184 & 0.86443 \\
\hline \multirow[t]{3}{*}{0.87094} & 0.00501 & -0.45302 & 0.07406 & -0.54851 & 0.11797 & 0.27751 \\
\hline & & -0.17774 & -0.34238 & 0.67053 & -0.02019 & -0.88718 \\
\hline & & 3682 & & 0.75 & 0.0 & 0.86410 \\
\hline \multirow[t]{3}{*}{0.87200} & 0.00988 & -0.44864 & 0.08155 & -0.55613 & 0.12536 & 0.30032 \\
\hline & 1388 & -0.18124 & -0.33791 & 0.66422 & -0.03294 & -0.87934 \\
\hline & 0.66250 & 3413 & 0.12083 & 0.75047 & 0.01680 & 0.86343 \\
\hline \multirow[t]{3}{*}{0.87234} & 0.01131 & -0.44740 & 0.08359 & -0.55816 & 0.12745 & 0.30680 \\
\hline & 1464 & -0.18211 & -0.33659 & 0.66250 & -0.03637 & -0.87696 \\
\hline & & & & 045 & 757 & 0.86319 \\
\hline \multirow[t]{3}{*}{0.87690} & 2599 & -0.43606 & 027 & -0.57470 & 566 & 0.36772 \\
\hline & & & & 837 & 309 & -0.85197 \\
\hline & 7621 & 0.22523 & 0.11464 & 0.75066 & 0.02520 & 0.86107 \\
\hline \multirow[t]{3}{*}{0.88144} & 0.03741 & -0.42805 & $\overline{926}$ & -0.58830 & 0.15623 & 0.41158 \\
\hline & & -0.18925 & -0.31251 & 0.63747 & -0.07550 & -0.83179 \\
\hline & 0.68597 & 21904 & 10960 & 0.75246 & 0.03011 & 0.86127 \\
\hline \multirow[t]{3}{*}{0.88322} & 0.04165 & -0.42461 & 0.11228 & -0.59631 & 0.16022 & 0.42848 \\
\hline & & -0.19073 & -0.30762 & 0.63068 & -0.07929 & -0.82356 \\
\hline & 0.69010 & 0.21667 & 0.10724 & 0.75334 & 0.03196 & 0.86185 \\
\hline
\end{tabular}

Table 4.10: Converged $\mathbf{S}$ matrix elements for singlet scattering with $\alpha=80, n_{\gamma}=4$, $R_{\max }=35$ au, $p_{\max }=8$, and $n_{R}^{e}=50$. 


\begin{tabular}{|c|c|c|c|c|c|c|}
\hline$E$ (Ryd) & $\begin{array}{r}\operatorname{Re} S_{11} \\
\operatorname{Im} S_{11} \\
\left|S_{11}\right|^{2}\end{array}$ & $\begin{array}{c}\operatorname{Re} S_{12} \\
\operatorname{Im} S_{12} \\
\left|S_{12}\right|^{2}\end{array}$ & $\begin{array}{c}\operatorname{Re} S_{13} \\
\operatorname{Im} S_{13} \\
\left|S_{13}\right|^{2}\end{array}$ & $\begin{array}{l}\operatorname{Re} S_{22} \\
\operatorname{Im} S_{22} \\
\left|S_{22}\right|^{2}\end{array}$ & $\begin{array}{c}\operatorname{Re} S_{23} \\
\operatorname{Im} S_{23} \\
\left|S_{23}\right|^{2}\end{array}$ & $\begin{array}{c}\operatorname{Re} S_{33} \\
\operatorname{Im} S_{33} \\
\left|S_{33}\right|^{2}\end{array}$ \\
\hline \multirow[t]{3}{*}{0.88348} & 0.04223 & -0.42409 & 0.11269 & -0.59766 & 0.16080 & 0.43085 \\
\hline & 0.83002 & -0.19101 & -0.30688 & 0.62949 & -0.07979 & -0.82238 \\
\hline & 0.69072 & 0.21634 & 0.10687 & 0.75346 & 0.03222 & 0.86194 \\
\hline \multirow[t]{3}{*}{0.88372} & 0.04282 & -0.42357 & 0.11311 & -0.59909 & 0.16139 & 0.43325 \\
\hline & 0.83035 & -0.19132 & -0.30613 & 0.62822 & -0.08029 & -0.82119 \\
\hline & 0.69131 & 0.21601 & 0.10651 & 0.75357 & 0.03249 & 0.86206 \\
\hline \multirow[t]{3}{*}{0.88396} & 0.04340 & -0.42303 & 0.11352 & -0.60061 & 0.16199 & 0.43567 \\
\hline & 0.83070 & -0.19167 & -0.30536 & 0.62685 & -0.08077 & -0.81997 \\
\hline & 0.69195 & 0.21569 & 0.10613 & 0.75367 & 0.03276 & 0.86216 \\
\hline \multirow[t]{3}{*}{0.88420} & 0.04399 & -0.42247 & 0.11393 & -0.60222 & 0.16261 & 0.43811 \\
\hline & 0.83104 & -0.19204 & -0.30457 & 0.62539 & -0.08125 & -0.81875 \\
\hline & 0.69256 & 0.21536 & 0.10574 & 0.75378 & 0.03304 & 0.86229 \\
\hline \multirow[t]{3}{*}{0.88446} & 0.04459 & -0.42190 & 0.11435 & -0.60394 & 0.16325 & 0.44057 \\
\hline & 0.83140 & -0.19245 & -0.30376 & 0.62380 & -0.08172 & -0.81750 \\
\hline & 0.6 & 0.21504 & 0.10535 & 0.75387 & 0.03333 & 0.86241 \\
\hline \multirow[t]{3}{*}{0.88470} & 0.04519 & -0.42132 & 0.11476 & -0.60578 & 0.16390 & 0.44307 \\
\hline & 0.83176 & -0.19290 & -0.30292 & 0.62209 & -0.08218 & -0.81623 \\
\hline & 0.69387 & 0.21472 & 0.10493 & 0.75397 & 0.03362 & 0.86254 \\
\hline \multirow[t]{3}{*}{0.88494} & 0.04579 & -0.42071 & 0.11517 & -0.60775 & 0.16457 & 0.44559 \\
\hline & 0.83212 & -0.19340 & -0.30206 & 0.62022 & -0.08263 & -0.81494 \\
\hline & 0.69452 & 0.21440 & 0.10450 & 0.75403 & 0.03391 & 0.86268 \\
\hline \multirow[t]{3}{*}{0.88518} & 0.04640 & -0.42009 & 0.11559 & -0.60988 & 0.16525 & 0.44814 \\
\hline & 0.83249 & -0.19396 & -0.30117 & 0.61819 & -0.08306 & -0.81364 \\
\hline & 0.69519 & 0.21410 & 0.10406 & 0.75411 & 0.03421 & 0.86284 \\
\hline \multirow[t]{3}{*}{0.88542} & 0.04702 & -0.41944 & 0.11600 & -0.61218 & 0.16596 & 0.45072 \\
\hline & 0.83286 & -0.19457 & -0.30026 & 0.61596 & -0.08347 & -0.81231 \\
\hline & 0.69587 & 0.21379 & 0.10361 & 0.75417 & 0.03451 & 0.86300 \\
\hline \multirow[t]{3}{*}{0.88568} & 0.04765 & -0.41877 & 0.11641 & -0.61467 & 0.16669 & 0.45334 \\
\hline & 0.83323 & -0.19525 & -0.29931 & 0.61351 & -0.08386 & -0.81095 \\
\hline & 0.69654 & 0.21349 & 0.10314 & 0.75421 & 0.03482 & 0.86316 \\
\hline
\end{tabular}

Table 4.11: Converged $\mathrm{S}$ matrix elements for singlet scattering with $\alpha=80, n_{\gamma}=4$, $R_{\max }=35$ au, $p_{\max }=8$, and $n_{R}^{e}=50$. 


\begin{tabular}{|c|c|c|c|c|c|c|}
\hline $\bar{E}(\mathrm{Ryd})$ & $\begin{array}{c}\operatorname{Re} S_{11} \\
\operatorname{Im} S_{11} \\
\left|S_{11}\right|^{2}\end{array}$ & $\begin{array}{c}\operatorname{Re} S_{12} \\
\operatorname{Im} S_{12} \\
\left|S_{12}\right|^{2}\end{array}$ & $\begin{array}{r}\operatorname{Re} S_{13} \\
\operatorname{Im} S_{13} \\
\left|S_{13}\right|^{2}\end{array}$ & $\begin{array}{c}\operatorname{Re} S_{22} \\
\operatorname{Im} S_{22} \\
\left|S_{22}\right|^{2}\end{array}$ & $\begin{array}{c}\operatorname{Re} S_{23} \\
\operatorname{Im} S_{23} \\
\left|S_{23}\right|^{2}\end{array}$ & $\begin{array}{l}\operatorname{Re} S_{33} \\
\operatorname{Im} S_{33} \\
\left|S_{33}\right|^{2}\end{array}$ \\
\hline \multirow[t]{3}{*}{0.88600} & 0.04851 & -0.41782 & 0.11696 & -0.61838 & 0.16771 & 0.45692 \\
\hline & 0.83374 & -0.19630 & -0.29799 & 0.60981 & -0.08432 & -0.80910 \\
\hline & 0.69748 & 0.21311 & 0.10248 & 0.75426 & 0.03524 & 0.86342 \\
\hline \multirow[t]{3}{*}{0.88742} & 0.05270 & -0.41292 & 0.11920 & -0.64197 & 0.17271 & 0.47341 \\
\hline & 0.83591 & -0.20356 & -0.29136 & 0.58470 & -0.08499 & -0.80056 \\
\hline & 0.70152 & 0.21194 & 0.09910 & 0.75400 & 0.03705 & 0.86501 \\
\hline \multirow[t]{3}{*}{0.88748} & 0.05291 & -0.41266 & 0.11928 & -0.64343 & 0.17296 & 0.47417 \\
\hline & 0.83600 & -0.20404 & -0.29103 & 0.58306 & -0.08493 & -0.80017 \\
\hline & 0.70170 & 0.21192 & 0.09893 & 0.75396 & 0.03713 & 0.86511 \\
\hline \multirow[t]{3}{*}{0.88754} & 0.05313 & -0.4 & & -0.64495 & 0.17320 & 0.47494 \\
\hline & 0.83608 & -0.20454 & -0.29070 & 0.58135 & -0.08486 & -0.79978 \\
\hline & 0.70185 & 0.21191 & 0.09875 & 0.75393 & 0.03720 & 0.86522 \\
\hline \multirow[t]{3}{*}{0.88760} & 0.05335 & -0.41213 & 0.11945 & -0.64652 & 0.17345 & 0.47572 \\
\hline & 0.83617 & -0.20506 & -0.29036 & 0.57956 & -0.08477 & -0.79939 \\
\hline & 0.70203 & 0.21190 & 0.09858 & 0.75388 & 0.03727 & 0.86533 \\
\hline \multirow[t]{3}{*}{0.88766} & 0.05357 & -0.41186 & 0.11952 & -0.64815 & 0.17370 & 0.47650 \\
\hline & 0.83625 & -0.20560 & -0.29001 & 0.57770 & -0.08467 & -0.79900 \\
\hline & 0.70218 & 0.21190 & 0.09839 & 0.75384 & 0.03734 & 0.86545 \\
\hline \multirow[t]{3}{*}{0.88772} & 0.05380 & -0.41157 & 0.11960 & -0.64985 & 0.17395 & 0.47728 \\
\hline & 0.83634 & -0.20617 & -0.28966 & 0.57575 & -0.08455 & -0.79860 \\
\hline & 0.70236 & 0.21190 & 0.09821 & 0.75379 & 0.03741 & 0.86556 \\
\hline \multirow[t]{3}{*}{0.88780} & 0.05403 & -0.41128 & 0.11967 & -0.65161 & 0.17420 & 0.47807 \\
\hline & 0.83642 & -0.20676 & -0.28930 & 0.57370 & -0.08442 & -0.79821 \\
\hline & 0.70252 & 0.21190 & 0.09802 & 0.75373 & 0.03747 & 0.86569 \\
\hline \multirow[t]{3}{*}{0.88786} & 0.05427 & -0.41099 & 0.11974 & -0.65344 & 0.17445 & 0.47887 \\
\hline & 0.83649 & -0.20738 & -0.28894 & 0.57156 & -0.08427 & -0.79781 \\
\hline & 0.70266 & 0.21192 & 0.09782 & 0.75366 & 0.03753 & 0.86582 \\
\hline \multirow[t]{3}{*}{0.88788} & 0.05438 & -0.41085 & 0.11978 & -0.65430 & 0.17457 & 0.47923 \\
\hline & 0.83653 & -0.20767 & -0.28878 & 0.57056 & -0.08419 & -0.79763 \\
\hline & 0.70274 & 0.21192 & 0.09774 & 0.75365 & 0.03756 & 0.86588 \\
\hline
\end{tabular}

Table 4.12: Converged $\mathbf{S}$ matrix elements for singlet scattering with $\alpha=80, n_{\gamma}=4$, $R_{\max }=35$ au, $p_{\max }=8$, and $n_{R}^{e}=50$. 


\begin{tabular}{|c|c|c|c|c|c|c|}
\hline$E(R y d)$ & $\begin{array}{c}\operatorname{Re} S_{11} \\
\operatorname{Im} S_{11} \\
\left|S_{11}\right|^{2}\end{array}$ & $\begin{array}{l}\operatorname{Re} S_{12} \\
\operatorname{Im} S_{12} \\
\left|S_{12}\right|^{2}\end{array}$ & $\begin{array}{c}\operatorname{Re} S_{13} \\
\operatorname{Im} S_{13} \\
\left|S_{13}\right|^{2}\end{array}$ & $\begin{array}{c}\operatorname{Re} S_{22} \\
\operatorname{Im} S_{22} \\
\left|S_{22}\right|^{2}\end{array}$ & $\begin{array}{r}\operatorname{Re} S_{23} \\
\operatorname{Im} S_{23} \\
\left|S_{23}\right|^{2}\end{array}$ & $\begin{array}{c}\operatorname{Re} S_{33} \\
\operatorname{Im} S_{33} \\
\left|S_{33}\right|^{2}\end{array}$ \\
\hline \multirow[t]{3}{*}{0.88790} & 0.05449 & -0.41071 & 0.11980 & -0.65514 & 0.17468 & 0.47958 \\
\hline & 0.83656 & -0.20796 & -0.28862 & 0.56957 & -0.08411 & -0.79746 \\
\hline & 0.70280 & 0.21193 & 0.09765 & 0.75362 & 0.03759 & 0.86594 \\
\hline \multirow[t]{3}{*}{0.88792} & 0.05452 & -0.41068 & 0.11981 & -0.65536 & 0.17471 & 0.47967 \\
\hline & 0.83657 & -0.20803 & -0.28858 & 0.56932 & -0.08409 & -0.79742 \\
\hline & 0.70282 & 0.21193 & 0.09763 & 0.75362 & 0.03759 & 0.86596 \\
\hline \multirow[t]{3}{*}{0.88794} & 0.05460 & -0.41058 & 0.11983 & -0.65599 & 0.17479 & 0.47993 \\
\hline & 0.83659 & -0.20825 & -0.28846 & 0.56857 & -0.08403 & -0.79729 \\
\hline & 0.70286 & 0.21194 & 0.09757 & 0.75359 & 0.03761 & 0.86600 \\
\hline \multirow[t]{3}{*}{0.88796} & 0.05471 & -0.41044 & 0.11986 & -0.65686 & 0.17491 & 0.48028 \\
\hline & 0.83663 & -0.20855 & -0.28829 & 0.56754 & -0.08395 & -0.79711 \\
\hline & 0.70294 & 0.21195 & 0.09748 & 0.75357 & 0.03764 & 0.86605 \\
\hline \multirow[t]{3}{*}{0.88798} & 0.05477 & -0.41037 & 0.11988 & -0.65735 & 0.17497 & 0.48048 \\
\hline & 0.83664 & -0.20872 & -0.28820 & 0.56696 & -0.08390 & -0.79702 \\
\hline & 0.70297 & 0.21197 & 0.09743 & 0.75355 & 0.03765 & 0.86610 \\
\hline \multirow[t]{3}{*}{0.88800} & 0.05482 & -0.41030 & 0.11989 & -0.65775 & 0.17502 & 0.48064 \\
\hline & 0.83666 & -0.20885 & -0.28813 & 0.56649 & -0.08386 & -0.79694 \\
\hline & 0.70301 & 0.21196 & 0.09739 & 0.75355 & 0.03766 & 0.86613 \\
\hline \multirow[t]{3}{*}{0.88802} & 0.05493 & -0.41016 & 0.11991 & -0.65865 & 0.17513 & 0.48099 \\
\hline & 0.83669 & -0.20916 & -0.28797 & 0.56541 & -0.08377 & -0.79677 \\
\hline & 0.70307 & 0.21198 & 0.09731 & 0.75351 & 0.03769 & 0.86619 \\
\hline \multirow[t]{3}{*}{0.88804} & 0.05503 & -0.41004 & 0.11994 & -0.65943 & 0.17523 & 0.48129 \\
\hline & 0.83672 & -0.20944 & -0.28782 & 0.56448 & -0.08369 & -0.79662 \\
\hline & 0.70313 & 0.21200 & 0.09723 & 0.75349 & 0.03771 & 0.86624 \\
\hline \multirow[t]{3}{*}{0.88808} & 0.05516 & -0.40988 & 0.11996 & -0.66050 & 0.17536 & 0.48170 \\
\hline & 0.83675 & -0.20981 & -0.28763 & 0.56319 & -0.08357 & -0.79642 \\
\hline & 0.70319 & 0.21202 & 0.09712 & 0.75344 & 0.03774 & 0.86632 \\
\hline \multirow[t]{3}{*}{0.88810} & 0.05528 & -0.40973 & 0.11999 & -0.66146 & 0.17547 & 0.48206 \\
\hline & 0.83678 & -0.21014 & -0.28746 & 0.56204 & -0.08346 & -0.79625 \\
\hline & 0.70326 & 0.21204 & 0.09703 & 0.75342 & 0.03776 & 0.86640 \\
\hline
\end{tabular}

Table 4.13: Converged $S$ matrix elements for singlet scattering with $\alpha=80, n_{\gamma}=4$, $R_{\max }=35$ au, $p_{\max }=8$, and $n_{R}^{e}=50$. 
between the $n=2$ and $n=3$ thresholds using the same scattering parameter values employed in the previous reporting of the individual $\mathbf{S}$ matrices. The resonance

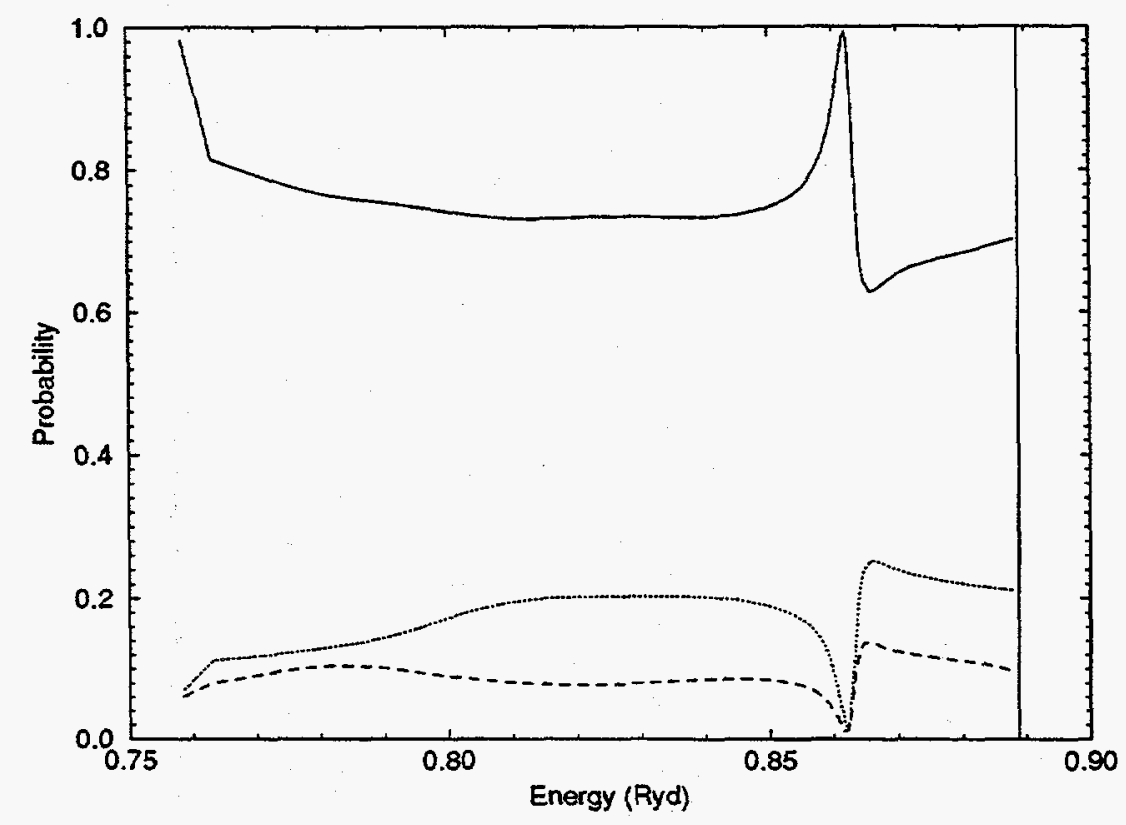

Figure 4.41: Converged transition probabilities $P_{1 x}$ for singlet scattering from $\phi_{1 s}$ state with $\alpha=80, n_{\gamma}=4, R_{\max }=35 \mathrm{au}, p_{\max }=8$, and $n_{R}^{e}=50$. Solid line: $\left|S_{11}\right|^{2}$; Dotted line: $\left|S_{12}\right|^{2}$; Dashed line: $\left|S_{13}\right|^{2}$. Vertical line: $n=3$ threshold.

feature located at $0.862 \mathrm{Ryd}$ has been resolved with a fine energy grid. It is apparent that within this energy region there is not much mixing between the $\phi_{1 s}$ state and the two degenerate $\phi_{2 s}$ and $\phi_{2 p}$ states. But there is a great deal of mixing between the $\phi_{2 s}$ and $\phi_{2 p}$ states, with transition probabilities between the two states being very dependent on the total energy of the system. Interestingly, there is a region just above 0.77 Ryd in which there is virtually no transition between these two degenerate states. Here, the $\mathbf{S}$ matrix is strongly diagonal. Results below 0.76 Ryd were found to be inaccurate due to poor unitarity and symmetry of the $\mathbf{S}$ matrix. Therefore, transition probabilities slightly above the $n=2$ threshold are not shown here.

Tables 4.14 through 4.16 show values of calculated $\mathrm{S}$ matrices for triplet scattering using the convergence parameters previously reported. Interestingly, it was found that achieving the same quality of unitarity for the triplet $\mathbf{S}$ matrix as for the singlet $\mathbf{S}$ matrix was more difficult. Errors in the triplet unitarity extended 


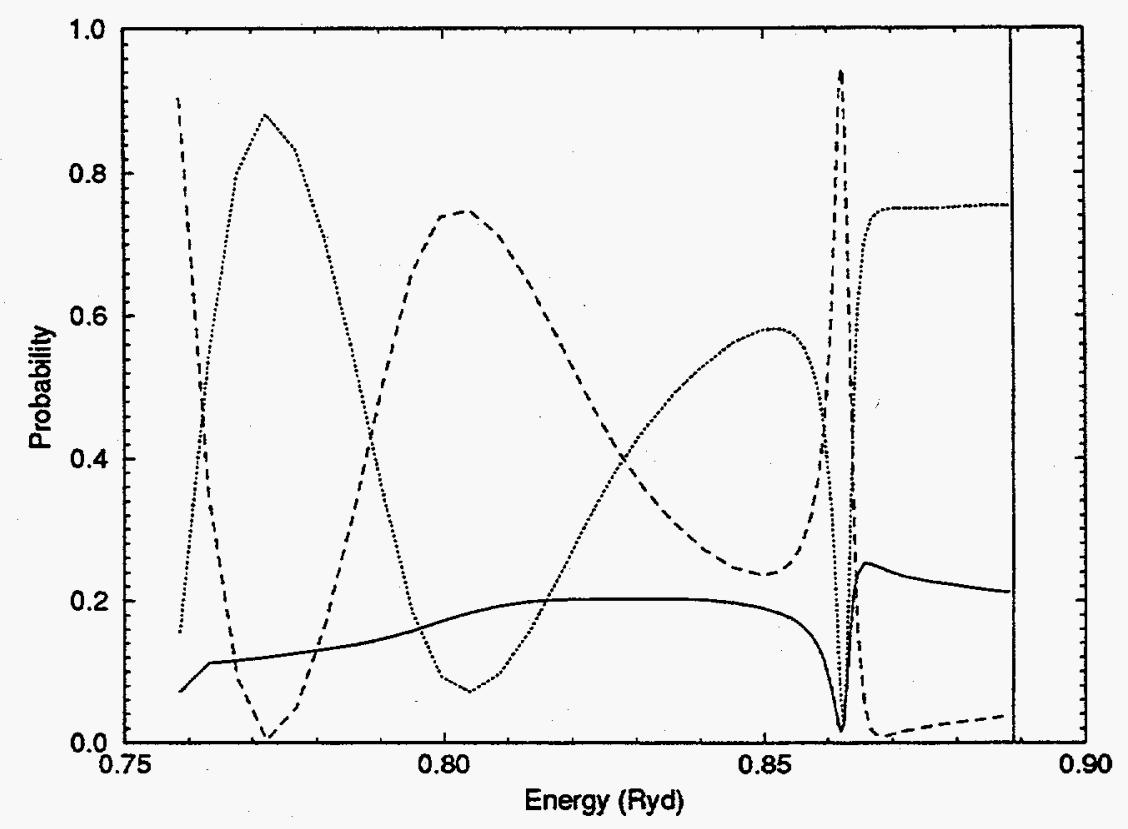

Figure 4.42: Converged transition probabilities $P_{2 x}$ for singlet scattering from $\phi_{2 s}$ state with $\alpha=80, n_{\gamma}=4, R_{\max }=35$ au, $p_{\max }=8$, and $n_{R}^{e}=50$. Solid line: $\left|S_{21}\right|^{2}$; Dotted line: $\left|S_{22}\right|^{2}$; Dashed line: $\left|S_{23}\right|^{2}$. Vertical line: $n=3$ threshold.

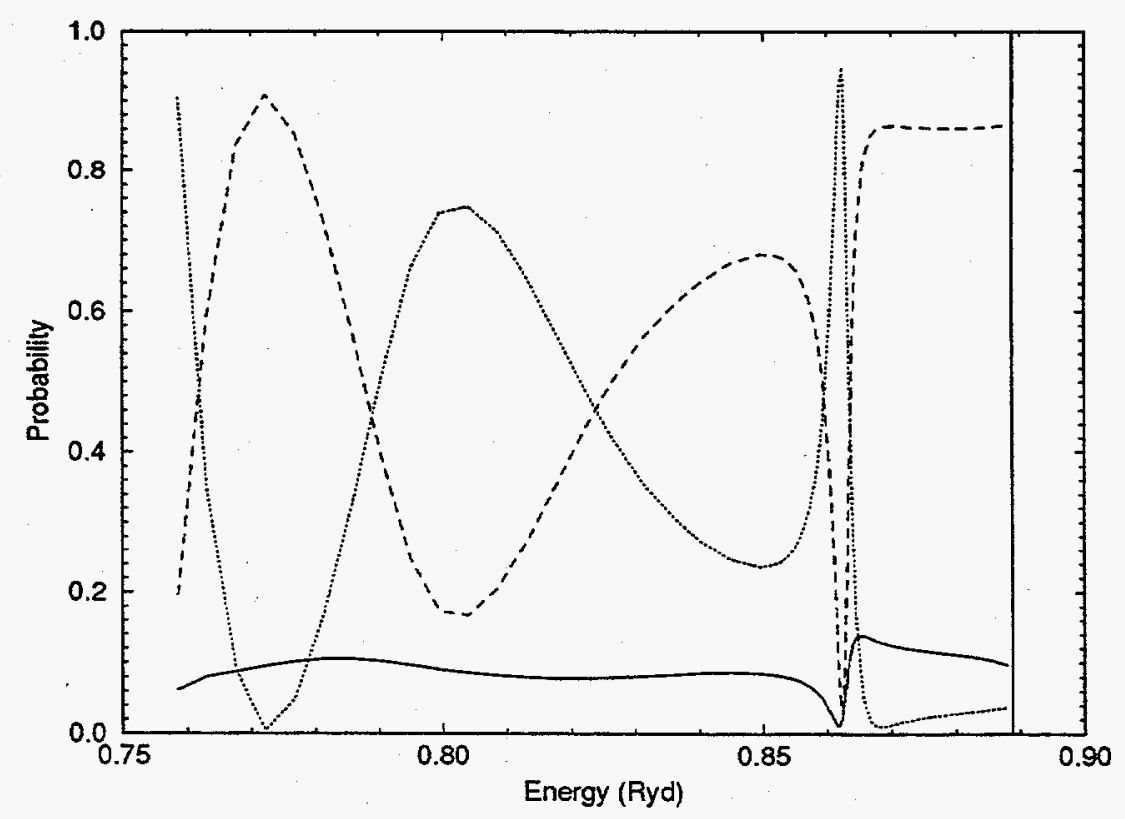

Figure 4.43: Converged transition probabilities $P_{3 x}$ for singlet scattering from $\phi_{2 p}$ state with $\alpha=80, n_{\gamma}=4, R_{\max }=35$ au, $p_{\max }=8$, and $n_{R}^{e}=50$. Solid line: $\left|S_{31}\right|^{2}$; Dotted line: $\left|S_{32}\right|^{2}$; Dashed line: $\left|S_{33}\right|^{2}$. Vertical line: $n=3$ threshold. 


\begin{tabular}{|c|c|c|c|c|c|c|}
\hline $\bar{E}(\mathrm{Ryd})$ & $\begin{array}{l}\operatorname{Re} S_{11} \\
\operatorname{Im} S_{11} \\
\left|S_{11}\right|^{2}\end{array}$ & $\begin{array}{l}\operatorname{Re} S_{12} \\
\operatorname{Im} S_{12} \\
\left|S_{12}\right|^{2}\end{array}$ & $\begin{array}{l}\operatorname{Re} S_{13} \\
\operatorname{Im} S_{13} \\
\left|S_{13}\right|^{2}\end{array}$ & $\begin{array}{c}\operatorname{Re} S_{22} \\
\operatorname{Im} S_{22} \\
\left|S_{22}\right|^{2}\end{array}$ & $\begin{array}{c}\operatorname{Re} S_{23} \\
\operatorname{Im} S_{23} \\
\left|S_{23}\right|^{2}\end{array}$ & $\begin{array}{r}\operatorname{Re} S_{33} \\
\operatorname{Im} S_{33} \\
\left|S_{33}\right|^{2}\end{array}$ \\
\hline \multirow[t]{3}{*}{0.75400} & -0.99856 & 0.01374 & -0.13154 & -0.40855 & 1.83792 & -0.74746 \\
\hline & 0.11547 & 0.15578 & 0.01537 & -2.64079 & -0.51728 & 2.31402 \\
\hline & 1.01046 & 0.02446 & 0.01754 & 7.14068 & 3.64553 & 5.91339 \\
\hline \multirow[t]{3}{*}{0.75856} & -1.00111 & 0.06888 & -0.16490 & -1.13417 & 0.83114 & -0.17078 \\
\hline & 0.10130 & 0.16642 & 0.01846 & -1.25745 & -1.19904 & 1.55523 \\
\hline & 1.01248 & 0.03244 & 0.02753 & 2.86752 & 2.12849 & 2.44791 \\
\hline \multirow[t]{3}{*}{0.76310} & -0.99504 & 0.01063 & -0.01552 & -0.08840 & -0.71895 & -0.22735 \\
\hline & 0.12343 & 0.02078 & 0.01043 & 0.24044 & -0.65187 & 0.11985 \\
\hline & 1.00534 & 0.00054 & 0.00035 & 0.06563 & 0.94182 & 0.06605 \\
\hline \multirow[t]{3}{*}{0.76766} & -0.99357 & 0.01439 & -0.01040 & 0.29462 & -0.80564 & -0.46841 \\
\hline & 0.12923 & 0.01440 & 0.01327 & 0.37145 & -0.37279 & 0.02612 \\
\hline & 1.00388 & 0.00041 & 0.00028 & 0.22478 & 0.78803 & 0.22009 \\
\hline \multirow[t]{3}{*}{0.77220} & -0.99212 & 0.01878 & -0.00757 & 0.59488 & -0.74083 & -0.65670 \\
\hline & 0.13481 & 0.01011 & 0.01520 & 0.30222 & -0.14009 & 0.07234 \\
\hline & 1.00248 & 0.00045 & 0.00029 & 0.44522 & 0.56845 & 0.43649 \\
\hline \multirow[t]{3}{*}{0.77676} & -0.99073 & 0.02287 & -0.00496 & 0.78601 & -0.61199 & -0.76959 \\
\hline & 0.14032 & & 0.01659 & 0.13627 & 0.01797 & 0.18649 \\
\hline & 1.00124 & 0.00056 & 0.00030 & 0.63638 & 0.37485 & 0.62705 \\
\hline \multirow[t]{3}{*}{0.78132} & -0.98943 & 0.02626 & -0.00226 & 0.88354 & -0.46264 & -0.82152 \\
\hline & 0.14577 & 0.00160 & 0.01754 & -0.05546 & 0.10081 & 0.31826 \\
\hline & 1.00022 & 0.00069 & 0.00031 & 0.78372 & 0.22420 & 0.77618 \\
\hline \multirow[t]{3}{*}{0.78586} & -0.98822 & 0.02880 & 0.00059 & 0.91170 & -0.31783 & -0.82777 \\
\hline & 0.15113 & -0.00267 & 0.01811 & -0.23866 & 0.12494 & 0.44513 \\
\hline & 0.99942 & 0.00084 & 0.00033 & 0.88816 & 0.11663 & 0.88334 \\
\hline \multirow[t]{3}{*}{0.79042} & -0.98713 & 0.03049 & 0.00356 & 0.89130 & -0.19035 & -0.80006 \\
\hline & 0.15638 & -0.00681 & 0.01836 & -0.39991 & 0.10806 & 0.55861 \\
\hline & 0.99888 & 0.00098 & 0.00035 & 0.95434 & 0.04791 & 0.95214 \\
\hline \multirow[t]{3}{*}{0.79496} & -0.98617 & 0.03143 & 0.00655 & 0.83894 & -0.08527 & -0.74867 \\
\hline & 0.16148 & -0.01073 & 0.01832 & -0.53416 & 0.06395 & 0.65454 \\
\hline & 0.99861 & 0.00110 & 0.00038 & 0.98915 & 0.01136 & 0.98893 \\
\hline
\end{tabular}

Table 4.14: Converged S matrix elements for triplet scattering with $\alpha=80, n_{\gamma}=4$, $R_{\max }=35 \mathrm{au}, p_{\max }=8$, and $n_{R}^{e}=50$. 


\begin{tabular}{|c|c|c|c|c|c|c|}
\hline $\mathrm{E}$ (Ryd) & $\begin{array}{l}\operatorname{Re} S_{11} \\
\operatorname{Im} S_{11} \\
\left|S_{11}\right|^{2}\end{array}$ & $\begin{array}{c}\operatorname{Re} S_{12} \\
\operatorname{Im} S_{12} \\
\left|S_{12}\right|^{2}\end{array}$ & $\begin{array}{c}\operatorname{Re} S_{13} \\
\operatorname{Im} S_{13} \\
\left|S_{13}\right|^{2}\end{array}$ & $\begin{array}{c}\operatorname{Re} S_{22} \\
\operatorname{Im} S_{22} \\
\left|S_{22}\right|^{2}\end{array}$ & $\begin{array}{c}\operatorname{Re} S_{23} \\
\operatorname{Im} S_{23} \\
\left|S_{23}\right|^{2}\end{array}$ & $\begin{array}{r}\operatorname{Re} S_{33} \\
\operatorname{Im} S_{33} \\
\left|S_{33}\right|^{2}\end{array}$ \\
\hline \multirow[t]{3}{*}{0.79952} & -0.98534 & 0.03174 & 0.00948 & 0.76719 & -0.00355 & -0.68217 \\
\hline & 0.16640 & -0.01437 & 0.01803 & -0.64102 & 0.00320 & 0.73149 \\
\hline & 0.99858 & 0.00121 & 0.00041 & 0.99949 & 0.00002 & 1.00043 \\
\hline \multirow[t]{3}{*}{0.80406} & -0.98464 & 0.03154 & 0.01227 & 0.68453 & 0.05589 & -0.60663 \\
\hline & 0.17111 & -0.01774 & 0.01750 & -0.72318 & -0.06608 & 0.79055 \\
\hline & 0.99879 & 0.00131 & 0.00046 & 0.99157 & 0.00749 & 0.99297 \\
\hline \multirow[t]{3}{*}{0.80862} & -0.98408 & 0.03096 & 0.01489 & 0.59631 & 0.09528 & -0.52614 \\
\hline & 0.17559 & -0.02087 & 0.01675 & -0.78436 & -0.13837 & 0.83384 \\
\hline & 0.99925 & 0.00139 & 0.00050 & 0.97081 & 0.02822 & 0.97211 \\
\hline \multirow[t]{3}{*}{0.81318} & -0.98365 & 0.03010 & 0.01730 & 0.50618 & 0.11731 & -0.44382 \\
\hline & 0.17984 & -0.02380 & 0.01578 & -0.82769 & -0.21029 & 0.86323 \\
\hline & 0.99991 & 0.00147 & 0.00055 & 0.94129 & 0.05798 & 0.94214 \\
\hline \multirow[t]{3}{*}{0.81772} & -0.98332 & 0.02906 & 0.01953 & 0.41705 & 0.12472 & -0.36239 \\
\hline & 0.18386 & -0.02658 & 0.01463 & -0.85551 & -0.27970 & 0.88020 \\
\hline & 1.00072 & 0.00155 & 0.00060 & 0.90583 & 0.09379 & 0.90608 \\
\hline \multirow[t]{3}{*}{0.82228} & -0.98307 & 0.02792 & 0.02158 & 0.33123 & 0.12002 & -0.28393 \\
\hline & 0.18768 & -0.02925 & 0.01332 & -0.86994 & -0.34505 & 0.88633 \\
\hline & 1.00165 & 0.00164 & 0.00064 & 0.86651 & 0.13346 & 0.86620 \\
\hline \multirow[t]{3}{*}{0.82682} & -0.98289 & 0.02673 & 0.02347 & 0.25029 & 0.10535 & -0.20966 \\
\hline & 0.19136 & -0.03183 & 0.01187 & -0.87311 & -0.40510 & 0.88338 \\
\hline & 1.00269 & 0.00173 & 0.00069 & 0.82497 & 0.17520 & 0.82432 \\
\hline \multirow[t]{3}{*}{0.83138} & -0.98275 & 0.02549 & 0.02524 & 0.17508 & 0.08255 & -0.14002 \\
\hline & 0.19494 & -0.03433 & 0.01032 & -0.86713 & -0.45908 & 0.87313 \\
\hline & 1.00380 & 0.00183 & 0.00074 & 0.78257 & 0.21757 & 0.78196 \\
\hline \multirow[t]{3}{*}{0.83594} & -0.98260 & 0.02421 & 0.02692 & 0.10598 & 0.05318 & -0.07505 \\
\hline & 0.19851 & -0.03678 & 0.00868 & -0.85386 & -0.50667 & 0.85699 \\
\hline & 1.00491 & 0.00194 & 0.00080 & 0.74031 & 0.25954 & 0.74006 \\
\hline \multirow[t]{3}{*}{0.84048} & -0.98241 & 0.02288 & 0.02853 & 0.04322 & 0.01865 & -0.01476 \\
\hline & 0.20215 & -0.03915 & 0.00697 & -0.83481 & -0.54793 & 0.83602 \\
\hline & 1.00599 & 0.00206 & 0.00086 & 0.69878 & 0.30058 & 0.69915 \\
\hline
\end{tabular}

Table 4.15: Converged $\mathrm{S}$ matrix elements for triplet scattering with $\alpha=80, n_{\gamma}=4$, $R_{\max }=35$ au, $p_{\max }=8$, and $n_{R}^{e}=50$. 


\begin{tabular}{|c|c|c|c|c|c|c|}
\hline$E$ (Ryd) & $\begin{array}{l}\operatorname{Re} S_{11} \\
\operatorname{Im} S_{11} \\
\left|S_{11}\right|^{2}\end{array}$ & $\begin{array}{r}\operatorname{Re} S_{12} \\
\operatorname{Im} S_{12} \\
\left|S_{12}\right|^{2}\end{array}$ & $\begin{array}{r}\operatorname{Re} S_{13} \\
\operatorname{Im} S_{13} \\
\left|S_{13}\right|^{2}\end{array}$ & $\begin{array}{r}\operatorname{Re} S_{22} \\
\operatorname{Im} S_{22} \\
\left|S_{22}\right|^{2}\end{array}$ & $\begin{array}{c}\operatorname{Re} S_{23} \\
\operatorname{Im} S_{23} \\
\left|S_{23}\right|^{2}\end{array}$ & $\begin{array}{r}\operatorname{Re} S_{33} \\
\operatorname{Im} S_{33} \\
\left|S_{33}\right|^{2}\end{array}$ \\
\hline \multirow[t]{3}{*}{0.84504} & -0.98215 & 0.02146 & 0.03011 & -0.01310 & -0.01975 & 0.04075 \\
\hline & 0.20593 & -0.04145 & 0.00520 & -0.81133 & -0.58308 & 0.81105 \\
\hline & 1.00703 & 0.00218 & 0.00093 & 0.65843 & 0.34037 & 0.65946 \\
\hline \multirow[t]{3}{*}{0.84958} & -0.98179 & 0.01994 & 0.03167 & -0.06302 & -0.06090 & 0.09142 \\
\hline & 0.20993 & -0.04365 & 0.00340 & -0.78466 & -0.61232 & 0.78289 \\
\hline & 1.00798 & 0.00230 & 0.00101 & 0.61966 & 0.37864 & 0.62127 \\
\hline \multirow[t]{3}{*}{0.85414} & -0.98129 & 0.01830 & 0.03321 & -0.10692 & -0.10380 & 0.13732 \\
\hline & 0.21424 & -0.04572 & 0.00159 & -0.75594 & -0.63585 & 0.75233 \\
\hline & 1.00883 & 0.00243 & 0.00111 & 0.58288 & 0.41508 & 0.58486 \\
\hline \multirow[t]{3}{*}{0.85868} & -0.98062 & 0.01653 & 0.03474 & -0.14539 & -0.14769 & 0.17865 \\
\hline & 0.21889 & -0.04762 & -0.00022 & -0.72611 & -0.65392 & 0.72009 \\
\hline & 1.00953 & 0.00254 & 0.00121 & 0.54837 & 0.44942 & 0.55045 \\
\hline \multirow[t]{3}{*}{0.86324} & -0.97977 & 0.01462 & 0.03623 & -0.17913 & -0.19200 & 0.21571 \\
\hline & 0.22394 & -0.04931 & -0.00202 & -0.69573 & -0.66691 & 0.68668 \\
\hline & 1.01010 & 0.00265 & 0.00132 & 0.51613 & 0.48163 & 0.51806 \\
\hline \multirow[t]{3}{*}{0.86780} & -0.97871 & 0.01260 & 0.03766 & -0.20872 & -0.23628 & 0.24867 \\
\hline & 0.22938 & -0.05079 & -0.00382 & -0.66508 & -0.67537 & 0.65245 \\
\hline & 1.01049 & 0.00274 & 0.00143 & 0.48590 & 0.51195 & 0.48753 \\
\hline \multirow[t]{3}{*}{0.87234} & -0.97745 & 0.01046 & 0.03904 & -0.23451 & -0.28018 & 0.27757 \\
\hline & 0.23521 & -0.05203 & -0.00560 & -0.63422 & -0.67986 & 0.61762 \\
\hline & 1.01073 & 0.00282 & 0.00156 & 0.45723 & 0.54071 & 0.45850 \\
\hline \multirow[t]{3}{*}{0.87690} & -0.97598 & 0.00824 & 0.04033 & -0.25668 & -0.32330 & 0.30240 \\
\hline & 0.24140 & -0.05304 & -0.00737 & -0.60328 & -0.68092 & 0.58251 \\
\hline & 1.01081 & 0.00288 & 0.00168 & 0.42983 & 0.56817 & 0.43076 \\
\hline \multirow[t]{3}{*}{0.88144} & -0.97431 & 0.00598 & 0.04154 & -0.27538 & -0.36514 & 0.32320 \\
\hline & 0.24790 & -0.05385 & -0.00912 & -0.57265 & -0.67893 & 0.54766 \\
\hline & 1.01073 & 0.00294 & 0.00181 & 0.40376 & 0.59427 & 0.40439 \\
\hline \multirow[t]{3}{*}{0.88600} & -0.97245 & 0.00374 & 0.04263 & -0.29095 & -0.40493 & 0.34017 \\
\hline & 0.25462 & -0.05445 & -0.01081 & -0.54307 & -0.67413 & 0.51410 \\
\hline & 1.01049 & 0.00298 & 0.00193 & 0.37958 & 0.61842 & 0.38001 \\
\hline
\end{tabular}

Table 4.16: Converged S matrix elements for triplet scattering with $\alpha=80, n_{\gamma}=4$, $R_{\max }=35 \mathrm{au}, p_{\max }=8$, and $n_{R}^{e}=50$. 
further in energy from the threshold regions near $n=2$ and $n=3$, and this is reflected in the tabulated results for the triplet $\mathbf{S}$ matrix. The same oscillatory pattern found in the unitarity of the singlet $\mathbf{S}$ matrix is manifested here also. The most striking feature of these triplet tables is the almost complete lack of transition from the $\phi_{1 s}$ state to either the $\phi_{2 s}$ state or $\phi_{2 p}$ state. One may then conclude that (at least within this energy region) the excitation of the hydrogen $\phi_{1 s}$ state must be accomplished by collision with an electron of opposite spin to that of the bound electron. This is very likely due to the fact that the Pauli exclusion principle prevents the scattering electron from penetrating the $\phi_{1 s}$ electron cloud. The bound 1 s electron then acts as a "hard target" during triplet scattering.

Figures 4.44 and 4.45 show the final values of the transition probabilities for triplet scattering from the $\phi_{2 s}$ and $\phi_{2 p}$ states for energies between the $n=2$ and $n=3$ thresholds using the same scattering parameter values employed in calculating the individual $\mathbf{S}$ matrices. (Transition probabilities for triplet scattering from the $\phi_{1 s}$ state are not shown here since $\left|S_{11}\right|^{2}$ is essentially unity and both $\left|S_{12}\right|^{2}$ and $\left|S_{13}\right|^{2}$ are vanishingly small.) There is an interesting region near $0.80 \mathrm{Ryd}$ where transitions from $\phi_{2 s}$ to $\phi_{2 p}$ (and vice versa) do not occur. This makes the triplet $\mathbf{S}$ matrix at this energy completely diagonal. It will be noticed that de-excitation from the $\phi_{2 s}$ and $\phi_{2 p}$ states into the $\phi_{1 s}$ state has a very low probability for the triplet symmetry. This, of course, is a natural consequence of the symmetry of the transition probability matrix $\mathbf{P}$ and the extremely low $\phi_{1 s}$ triplet excitation probabilities described previously.

\subsubsection{Cross Sections}

Figures 4.46 and 4.47 show elastic and inelastic cross sections composed of properly statistically weighted singlet and triplet contributions. The formula for the cross section is given by

$$
\sigma_{i j}=\frac{\pi}{k_{i}^{2}}\left(\frac{1}{4}\left|\delta_{i j}-{ }^{1} S_{i j}\right|^{2}+\frac{3}{4}\left|\delta_{i j}-{ }^{3} S_{i j}\right|^{2}\right)
$$

where the superscripts " 1 " and " 3 " indicate singlet and triplet respectively. In the elastic scattering case, one can see that the sharpness of the Feshbach resonance near 


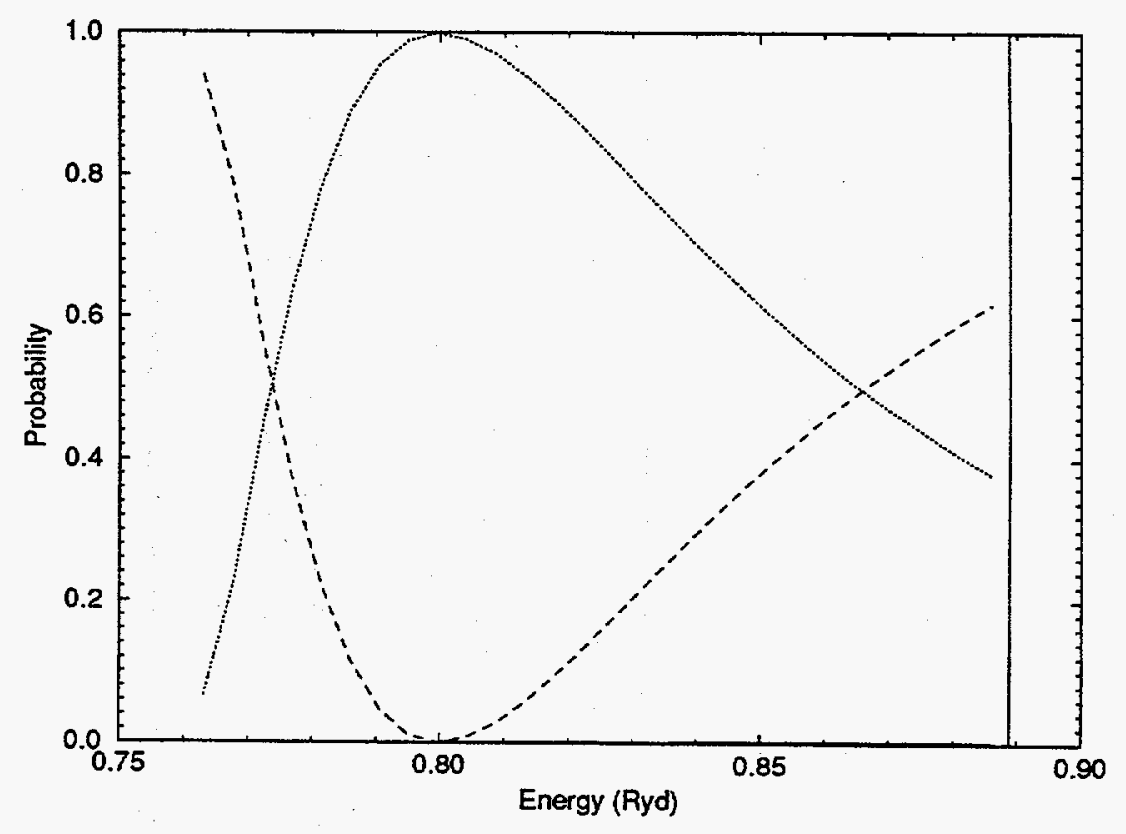

Figure 4.44: Converged transition probabilities $P_{2 x}$ for triplet scattering from $\phi_{2 s}$ state with $\alpha=80, n_{\gamma}=4, R_{\max }=35$ au, $p_{\max }=8$, and $n_{R}^{e}=50$. Solid line: $\left|S_{21}\right|^{2}$; Dotted line: $\left|S_{22}\right|^{2}$; Dashed line: $\left|S_{23}\right|^{2}$. Vertical line: $n=3$ threshold. The solid line is barely distinguishable from the energy axis. 


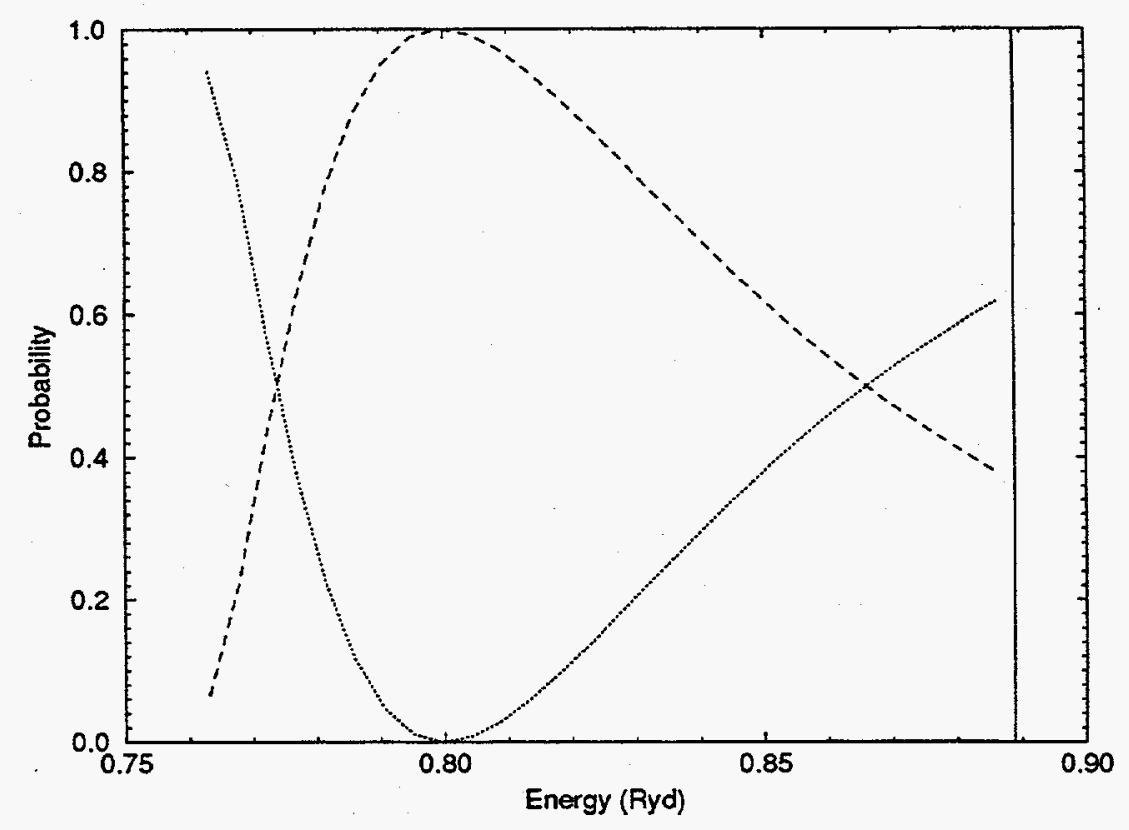

Figure 4.45: Converged transition probabilities $P_{3 x}$ for triplet scattering from $\phi_{2 p}$ state with $\alpha=80, n_{\gamma}=4, R_{\max }=35$ au, $p_{\max }=8$, and $n_{R}^{e}=50$. Solid line: $\left|S_{31}\right|^{2}$; Dotted line: $\left|S_{32}\right|^{2}$; Dashed line: $\left|S_{33}\right|^{2}$. Vertical line: $n=3$ threshold. The solid line is barely distinguishable from the energy axis.

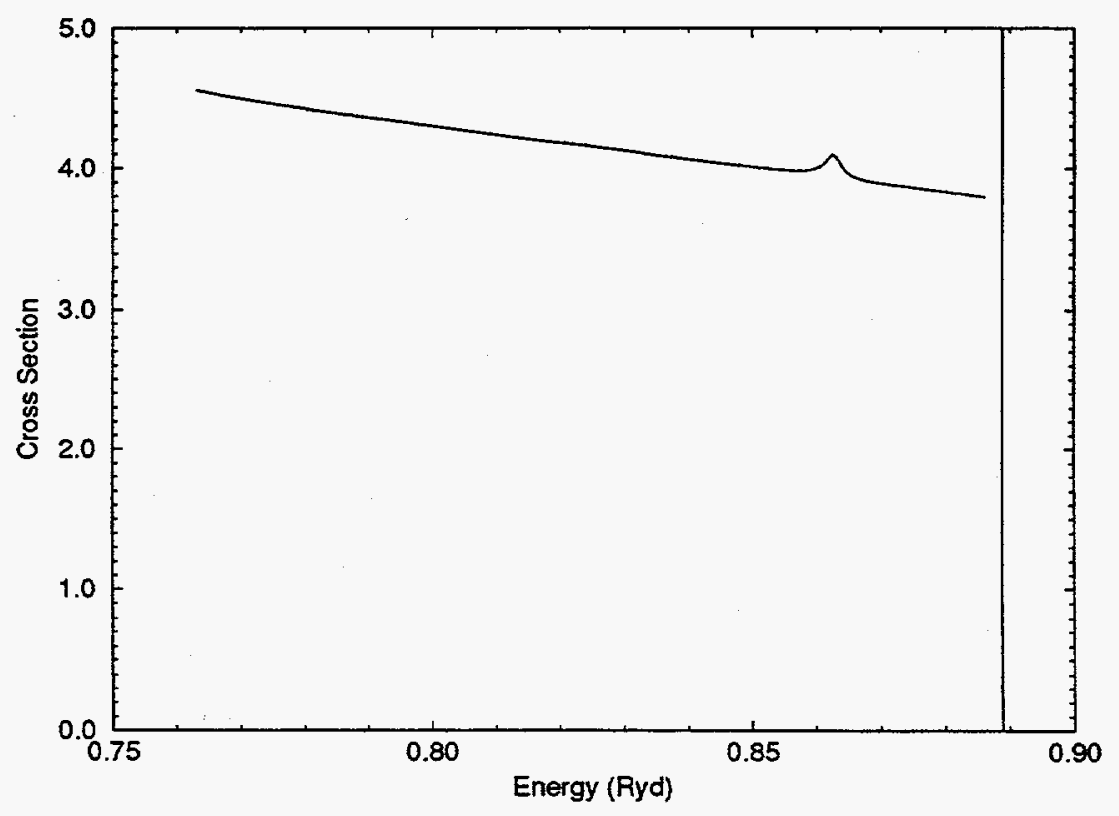

Figure 4.46: Elastic cross section $\sigma_{1 s \rightarrow 1 s}$ (in units of $\pi a_{0}^{2}$ ) derived from singlet and triplet calculations with $\alpha=80, n_{\gamma}=4, R_{\max }=35 \mathrm{au}, p_{\max }=8$, and $n_{R}^{e}=50$. Vertical line: $n=3$ threshold. 


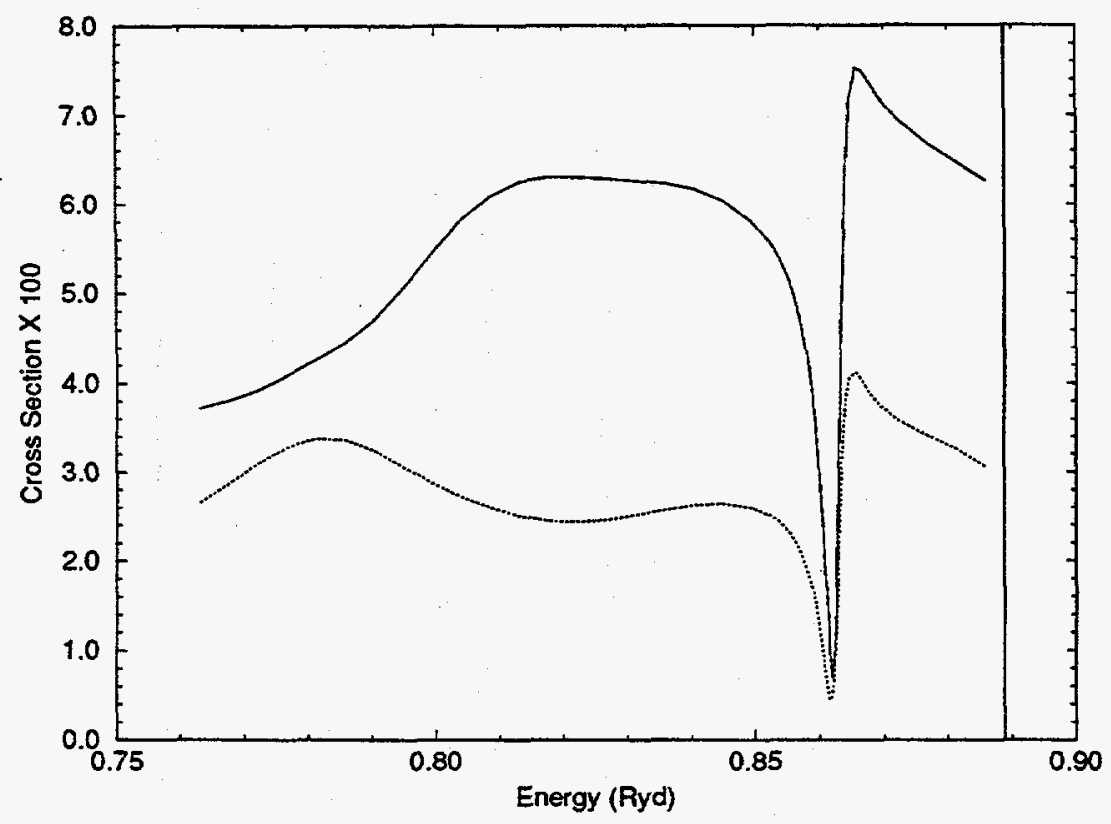

Figure 4.47: Inelastic cross sections $\sigma_{1 s \rightarrow 2 s}$ and $\sigma_{1 s \rightarrow 2 p}$ (in units of $\pi a_{0}^{2}$ ) derived from singlet and triplet calculations with $\alpha=80, n_{\gamma}=4, R_{\max }=35$ au, $p_{\max }=8$, and $n_{R}^{e}=50$. Solid line: $\sigma_{1 s \rightarrow 2 s}$; Dotted line: $\sigma_{1 s \rightarrow 2 p}$; Vertical line: $n=3$ threshold.

0.862 Ryd has been smoothed out by the triplet contribution, leaving only a small deviation from a nearly straight line. But this sharpness is retained in the case of inelastic scattering.

\subsection{5 "Distinguishable" Electrons}

In order to gain some insight into the effect of symmetry constraints on the results of these calculations, it is instructive to examine the hydrogen states resulting from the scattering of "distinguishable" electrons whose total wavefunctions (spatial and spin) are not subject to the constraint of being anti-symmetric. A simple linear combination of singlet and triplet scattering wavefunctions provides the correct formulae for transition probabilities pertaining to distinguishable (albeit non-physical) electrons.

$$
\begin{aligned}
& P_{i j}^{d i r}=\frac{1}{4}\left|{ }^{1} S_{i j}+{ }^{3} S_{i j}\right|^{2}, \quad \text { and } \\
& P_{i j}^{e x}=\frac{1}{4}\left|{ }^{1} S_{i j}-{ }^{3} S_{i j}\right|^{2}
\end{aligned}
$$


where $P_{i j}^{d i r}$ and $P_{i j}^{e x}$ are the direct and exchange scattering transition probabilities respectively, and ${ }^{1} S_{i j}$ and ${ }^{3} S_{i j}$ are singlet and triplet $\mathbf{S}$ matrix elements.

One of the first features which is noticeable is the nearly constant value of $P_{11}^{\text {dir }}$ throughout the entire energy region in Figure 4.48 except for the small area around the resonance at $0.862 \mathrm{Ryd}$. This is in contrast to the singlet scattering results

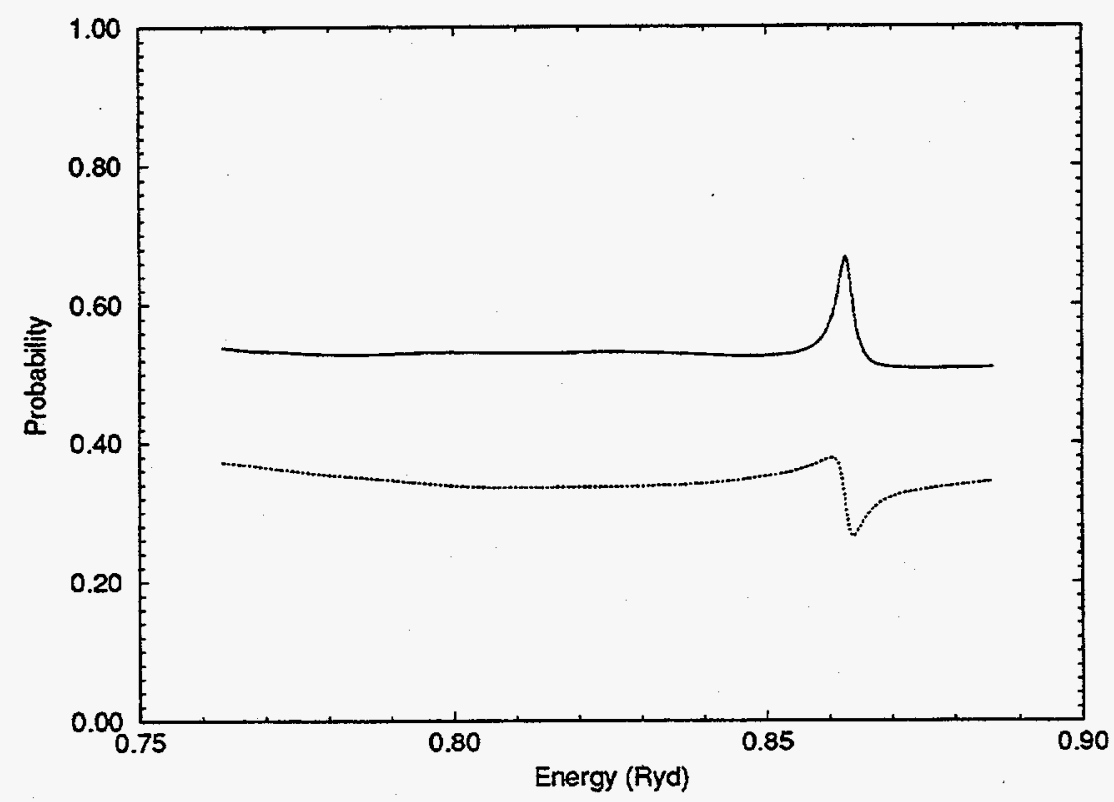

Figure 4.48: Direct and exchange transition probabilities $P_{11}^{d i r}$ and $P_{11}^{e x}$ for distinguishable elastic $e^{-}$scattering from $\phi_{1 s}$ state. Solid line: $P_{11}^{d i r}$; Dotted line: $P_{11}^{e x}$.

seen in Figure 4.41 where there is slightly more variation in the transition probability.

Figures $4.49,4.50$, and 4.51 demonstrate how the importance of electron exchange varies greatly for scattering between the two $n=2$ hydrogen states. It is especially seen to be important in the resonance region. It is particularly interesting to note that there is a peculiar oscillation in the direct and exchange contributions to the transition probabilities. (This is particularly noticeable in the $\phi_{2 p} \rightarrow \phi_{2 p}$ transitions shown in Figure 4.51.)

Another interesting feature is the near perfect destructive interference between the direct and exchange contributions to the $\phi_{2 s} \rightarrow \phi_{2 p}$ transition probability at 0.80 Ryd, shown in Figure 4.50. This causes the triplet contribution to this transition to completely vanish (making the triplet $\mathbf{S}$ matrix almost completely diagonal at 


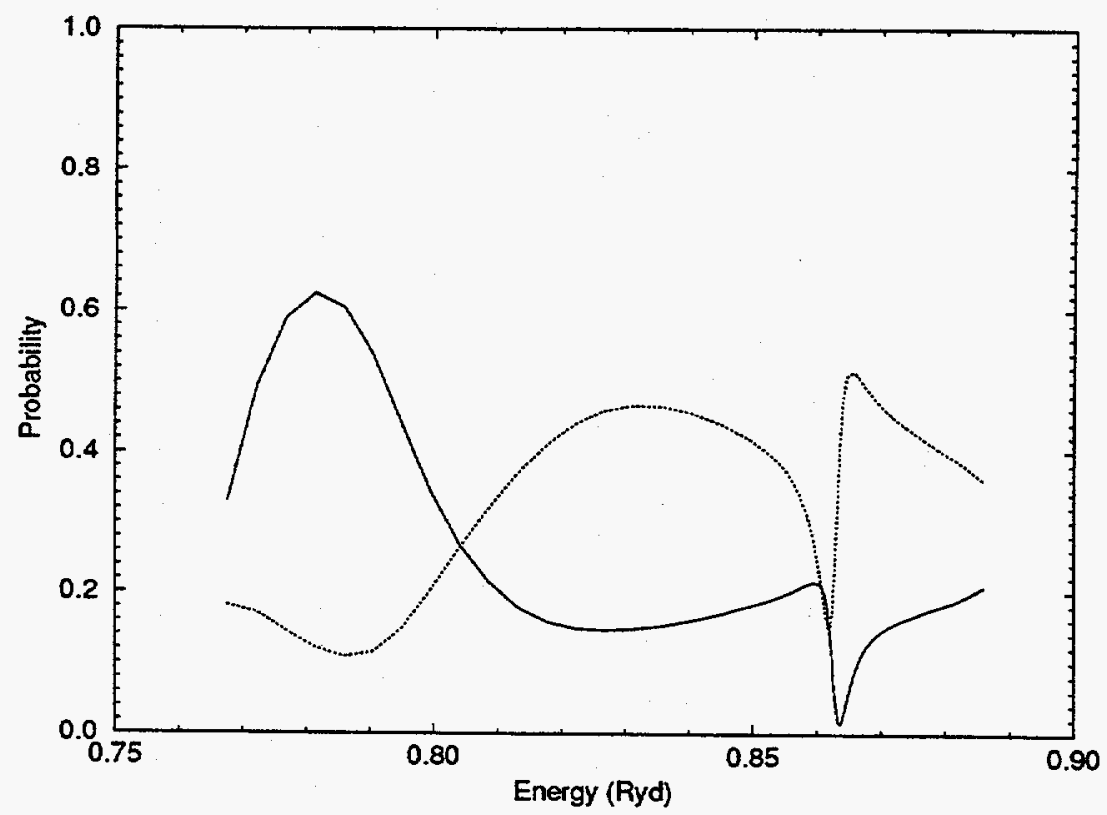

Figure 4.49: Direct and exchange transition probabilities $P_{22}^{\text {dir }}$ and $P_{22}^{e x}$ for distinguishable elastic $e^{-}$scattering from $\phi_{2 s}$ state. Solid line: $P_{22}^{\text {dir }}$; Dotted line: $P_{22}^{e x}$.

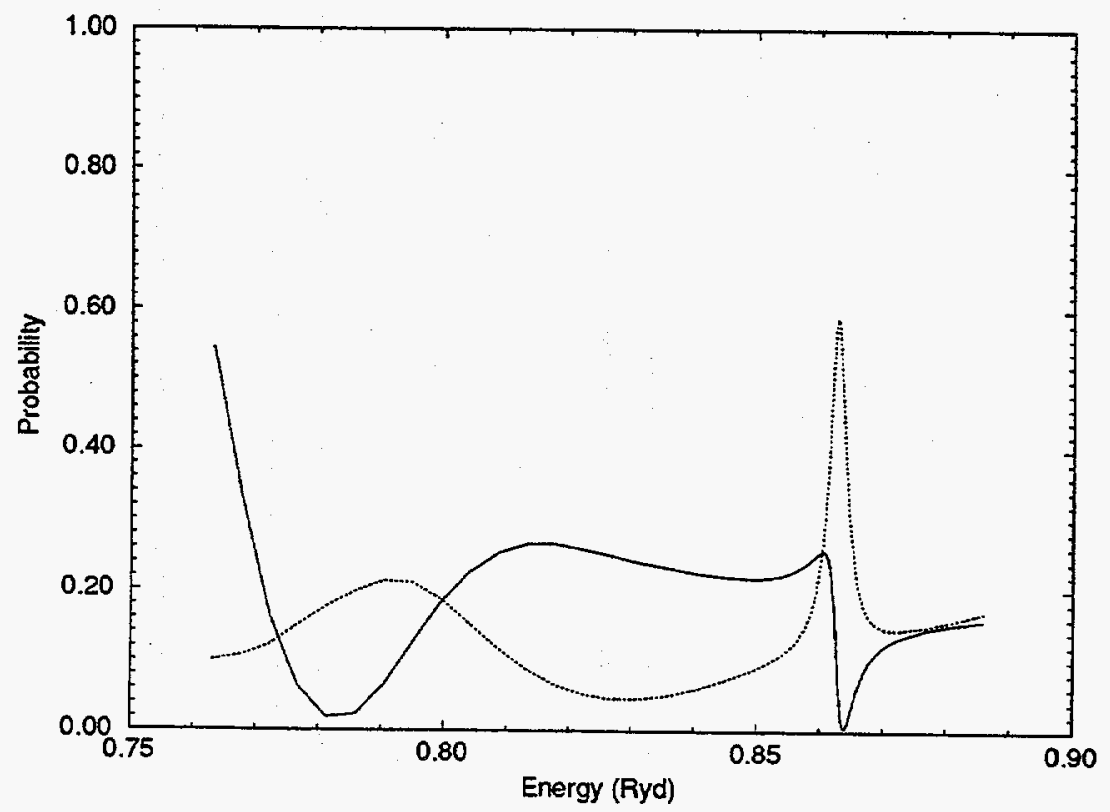

Figure 4.50: Direct and exchange transition probabilities $P_{23}^{\text {dir }}$ and $P_{23}^{e x}$ for distinguishable inelastic $e^{-}$scattering from $\phi_{2 s}$ state to $\phi_{2 p}$ state. Solid line: $P_{23}^{\text {dir }} ;$ Dotted line: $P_{23}^{e x}$. 


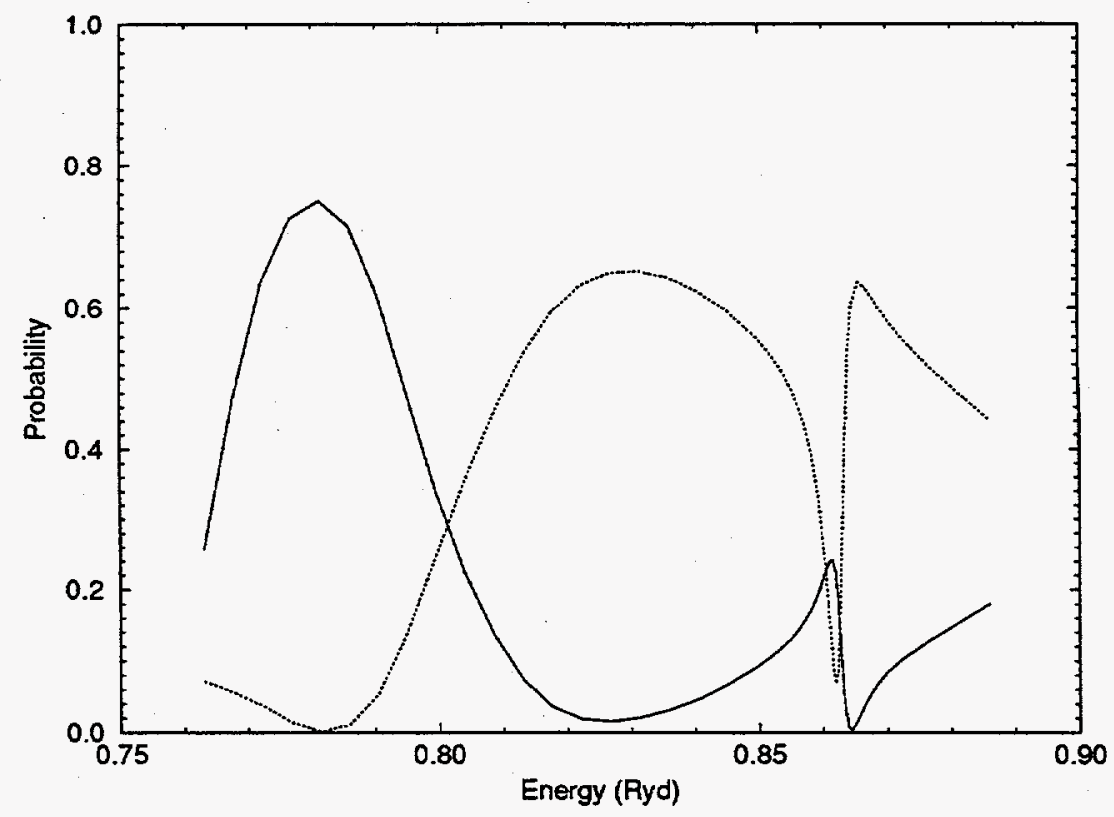

Figure 4.51: Direct and exchange transition probabilities $P_{33}^{\text {dir }}$ and $P_{33}^{e x}$ for distinguishable elastic $e^{-}$scattering from $\phi_{2 p}$ state. Solid line: $P_{33}^{\text {dir }}$; Dotted line: $P_{33}^{e x}$.

this energy). It is also interesting to see that the rather complicated structure seen in the direct and exchange $\phi_{2 s} \rightarrow \phi_{2 p}$ transition probabilities results in a rather simple triplet transition probability structure in Figure 4.44.

\subsection{Comparisons with other Theoretical Results}

\subsubsection{Cross Section Comparisons}

In this section, the results from the present potential-optimized DVR are compared with other theoretical studies which have examined $J_{\text {total }}=0$ electron scattering from a hydrogen atom at total energies between the $n=2$ and $n=3$ thresholds. A comparison of singlet cross sections is first performed. Table 4.17 shows a comparison of the present DVR results with calculations performed by Callaway [97] and Wang and Callaway [69] respectively. These results show calculated elastic and excitation cross sections for singlet scattering at five representative energies between the $n=2$ and $n=3$ thresholds. 


\begin{tabular}{||c||c|c|c|c|}
\hline$k^{2}$ (Ryd) & Study & $\sigma_{1 s \rightarrow 1 s}$ & $\sigma_{1 s \rightarrow 2 s}$ & $\sigma_{1 s \rightarrow 2 p}$ \\
\hline 0.76000 & C & 0.663 & 0.0373 & 0.0281 \\
& WC & 0.678 & 0.0381 & 0.0265 \\
& DVR & 0.664 & 0.0328 & 0.0233 \\
\hline 0.78000 & C & 0.611 & 0.0411 & 0.0349 \\
& WC & 0.627 & 0.0434 & 0.0349 \\
& DVR & 0.598 & 0.0416 & 0.0334 \\
\hline 0.80000 & C & 0.578 & 0.0534 & 0.0294 \\
& WC & 0.581 & 0.0545 & 0.0285 \\
& DVR & 0.576 & 0.0539 & 0.0282 \\
\hline 0.81000 & C & 0.562 & 0.0591 & 0.0257 \\
& WC & 0.561 & 0.0578 & 0.0279 \\
& DVR & 0.562 & 0.0603 & 0.0252 \\
\hline 0.82000 & C & 0.547 & 0.0616 & 0.0245 \\
& WC & 0.557 & 0.0584 & 0.0265 \\
& DVR & 0.551 & 0.0617 & 0.0238 \\
\hline
\end{tabular}

Table 4.17: Comparison of singlet excitation cross sections (in units of $\pi a_{0}^{2}$ ) for different theoretical methods. C: Variational close-coupling calculation by Callaway [97]; WC: Direct numerical calculation by Wang and Callaway [69]; DVR: Present Potential-optimized DVR method. All cross sections contain the proper statistical weighting factor for singlet scattering. 
The two Callaway calculations are very different in character. The earlier calculation by Callaway utilizes a more conventional variational calculation which expands the wavefunction in a basis of pseudo-states. A basis of $7 \mathrm{~s}, 5 \mathrm{p}, 3 \mathrm{~d}, 2 \mathrm{f}$, and $1 \mathrm{~g}$ states are used to describe the hydrogen atom. This basis includes exact hydrogenic eigenfunctions through $n=3$ plus the exact atomic $4 \mathrm{f}$ state. Eleven other pseudostates $(4 \mathrm{~s}, 3 \mathrm{p}, 2 \mathrm{~d}, 1 \mathrm{f}$, and $1 \mathrm{~g})$ are also included. The parameters describing the entire basis are given in Table 7 of Callaway [12].

The later calculation by Wang and Callaway utilizes a direct numerical approach to the solving of the Schrödinger equation in which the total wavefunction is expanded in angular states (up to $l=3$ for the individual electrons), symmetrized according to spin, and the remaining two radial degrees of freedom solved on a grid of equally-spaced points through a Numerov propagation mechanism. (A detailed description of the method is provided by Poet [68].) For the particular results quoted, the Numerov propagation was performed out to a value of the radial coordinate $R_{\max }=40$ au with a step size $h=0.2$. The $\mathrm{S}$ matrix is then determined by a least-squares fit with known asymptotic solutions provided by the method of Burke and Schey [89]. It will be noted that these are the same solutions which provide asymptotic functions in this present DVR method.

It is immediately apparent that the closest agreement of the present method is with the earlier variational close-coupling method of Callaway. This is perhaps not very surprising in light of the fact that the basic equations in the present DVR method are variational in character also. Callaway also includes in his basis set functions of the form described by (3.101). This can be thought of as partially substituting for the reciprocal $R$ expansion of Burke and Schey [89] used in the present DVR method. Although the present DVR method is derived from equations which are variational in character, it must be pointed out that there can be no rigorous variational principle associated with the resulting calculation. This is because the potential energy matrix elements calculated in this method are necessarily a quadrature approximation in the spirit of the method derived by Harris, Engerholm, and Gwinn [25]. Of course, in the limit of a large set of closely-spaced, localized DVR functions true variational character will be approached as the quadrature approximation improves. In all, the 
cross sections calculated by the present method appear to duplicate the results of the two Callaway calculations to a large degree.

\subsubsection{Eigenphase Sum Comparisons}

A better comparison of different methods is achieved through calculation of the eigenphase sum. A diagonalization of the calculated $\mathbf{S}$ matrix yields a sequence of diagonal elements each of which is associated with a single open channel. These values can be placed in the form

$$
\tilde{S}_{i i}=e^{2 i \tilde{\delta}_{i i}}
$$

where the index $i$ is an open channel index and the tilde indicates diagonalization. The sum $\sum_{i} \tilde{\delta}_{i i}$ is then the eigenphase sum. It will be noticed that an arbitrary integral number of terms $\pi$ can be added to or subtracted from $\tilde{\delta}_{i i}$ while $\tilde{S}_{i i}$ will not change. A unique convention must be established for deriving a unique $\tilde{\delta}_{i i}$ from a value of $\tilde{S}_{i i}$ in order for the concept of an eigenphase to have meaning.

The usefulness of the eigenphase sum as a measure of the effectiveness of a particular calculation is based on the fact that the exact values of the tangents of the eigenphases are upper bounds on the same quantities calculated in close-coupling calculations in which exact target wavefunctions are present in all open channels [4, p.132]. The size of the eigenphase sum can therefore be thought of as a measure of the quality of the scattering calculation.

Table 4.18 compares the singlet eigenphase sums calculated with the present DVR method to those calculated with the direct numerical (Numerov) method of Wang and Callaway [69], the conventional variational close-coupling method of Callaway [97], and the $R$ matrix propagation methods of Taylor and Burke [98] and Geltman and Burke [99]. The two calculations of Callaway are described previously in the comparison of singlet cross sections.

Both of the Burke calculations use the $\mathrm{R}$ matrix propagation method which is described in some detail in a lengthy review article by Burke and Smith [11]. Generally, the method involves transforming the Schrödinger equation for the electronhydrogen system into a set of coupled-channel equations through the conventional 


\begin{tabular}{||c||r|r|r|r|r||}
\hline \multirow{2}{*}{$k^{2}$ (Ryd) } & \multicolumn{5}{|c||}{$\sum_{i} \tilde{\delta}_{i i}$} \\
\cline { 2 - 6 } & DVR & C-NUM & C-VAR & \multicolumn{1}{c|}{ TB } & \multicolumn{1}{c|}{ GB } \\
\hline 0.76000 & -0.464 & -0.434 & -0.455 & -0.508 & -0.535 \\
0.78000 & 1.697 & 1.714 & 1.706 & 1.640 & 1.620 \\
0.80000 & 1.645 & 1.657 & 1.625 & & \\
0.81000 & 1.686 & 1.673 & 1.659 & 1.618 & 1.559 \\
0.82000 & 1.719 & 1.687 & 1.675 & & \\
0.83000 & 1.701 & & & 1.615 & 1.562 \\
0.85000 & 1.695 & 1.695 & 1.665 & & \\
0.85500 & 1.749 & 1.806 & 1.726 & & \\
0.86000 & 2.037 & 2.241 & 2.127 & & \\
0.86200 & 2.626 & 3.094 & 3.085 & & \\
\hline
\end{tabular}

Table 4.18: Comparison of singlet eigenphase sums. DVR: Present results; C-NUM: Direct numerical (Numerov) results of Wang and Callaway [69]; C-VAR: Variational close-coupling results of Callaway [97]; TB: $\mathrm{R}$ matrix propagation method of Taylor and Burke [98]; GB: R matrix propagation method of Geltman and Burke [99].

expansion of the total wavefunction in terms of hydrogen eigenstates supplimented by various types of pseudo-states in order to attain some measure of completeness. The resulting coupled differential equations are then solved by numerical integration from the origin step by step (by a Runge-Kutta algorithm or other techniques). Because closed channels may be included in the coupled-channel expansion, the propagation of the coefficient matrix is stopped before the non-physical rising exponential solutions associated with the closed channels become too large. A second propagation is started in the asymptotic region, and the resulting wavefunction is matched to the previously propagated solution at some convenient value of $R$. $\left(0^{\text {th }}\right.$ and $1^{\text {st }}$ order derivatives are matched.) Explicitly symmetrizing the form of the total wavefunction to include electron exchange effects transforms the resulting equations into integro-differential equations which are then solved iteratively until self-consistency of a desired degree is attained.

The calculation of Geltman and Burke [99] used only six states in the coupled-channel expansion - the exact $1 s, 2 s$, and $2 p$ eigenstates of hydrogen and three pseudo-states. The calculation of Taylor and Burke [98] used the lowest six exact hydrogen eigenstates (through the $3 \mathrm{~d}$ state) together with variationally-determined 
Hylleraas-type correlation terms given by

$$
\phi_{i}^{L M_{L}}\left(\mathbf{r}_{1} \mathbf{r}_{2}\right)=r_{1}^{p_{i}} r_{2}^{q_{i}} r_{12}^{s_{i}} \exp \left\{-\frac{1}{2} K\left(r_{1}+r_{2}\right)\right\} \mathcal{Y}_{L l_{i_{i}} l_{2_{i}}}^{M_{L}}\left(\hat{\mathbf{r}}_{1} \hat{\mathbf{r}}_{2}\right)
$$

where $K$ is a length scale parameter and

$$
\mathcal{Y}_{L l_{1} l_{2}}^{M M_{x}}\left(\hat{\mathbf{r}}_{1} \hat{\mathbf{r}}_{2}\right)=\sum_{m_{l_{1}} m_{l_{2}}} C\left(l_{1} l_{2} L ; m_{l_{1}} m_{l_{2}} M_{L}\right) Y_{l_{1}}^{m_{l_{1}}}\left(\hat{\mathbf{r}}_{1}\right) Y_{l_{2}}^{m_{l_{2}}}\left(\hat{\mathbf{r}}_{2}\right) \text {. }
$$

All exponents are integers and provide powers of $r_{1}$ and $r_{2}$ which satisfy the proper boundary conditions at the origin for their corresponding values of $l_{1}$ and $l_{2}$. (These are the same types of functions used by Schwartz [18].)

Examination of the comparison between these different methods indicates that the present DVR method achieves the most improvement over the other methods at the intermediate range energies which are furthest from the threshold regions. For example, just above the $n=2$ threshold the DVR method does not attain as large an eigenphase sum as the two Callaway calculations. This can be explained by the fact that the reciprocal $R$ expansion forming the asymptotic form of the total wavefunction is weakest just above and just below thresholds, energy regions which will therefore require an $L^{2}$ basis set to span a wider range of the scattering coordinate. (Wang and Callaway [69] comment that terms beyond $R^{-3}$ have little effect on results except at $k^{2}=0.76$ Ryd.) Beyond the $n=2$ threshold the DVR method achieves equally good or better eigenphase sums up until the energy enters the Feshbach resonance region around 0.862 Ryd. Within this resonance region the results are not as good as those achieved by the two Callaway calculations. (This mirrors the previously noted lack of convergence in the value of the $\mathbf{S}$ matrix in this energy region.)

It should be noted that the direct numerical (Numerov) calculation performed by Callaway carried the propagation up to a value of $R=40$ au in the scattering coordinate. The present DVR calculation placed the center of the furthest DVR basis function at $R=35 \mathrm{au}$. This fact may account for inaccuracies at energies just above the $n=2$ threshold due to the increase in size of the interaction region caused by weakness in the reciprocal $R$ expansion. Lack of convergence within the Feshbach resonance region may indicate that the spatial extent of a resonant virtual state extends beyond the maximum value for $R$ spanned by the DVR basis functions. 
Table 4.19 compares the triplet eigenphase sums calculated with the present DVR method to those calculated with the 18-state variational close-coupling method of Callaway [97], the $R$ matrix propagation methods of Taylor and Burke [98] and Geltman and Burke [99], and the 11-state variational close-coupling method of Callaway and Wooten [87]. Each of these methods has been described, with the exception

\begin{tabular}{||c||c|c|c|c|c||}
\hline \multirow{2}{*}{$k^{2}$ (Ryd) } & \multicolumn{5}{c||}{$\sum_{i} \tilde{\delta}_{i i}$} \\
\cline { 2 - 6 } & DVR & C & TB & GB & CW \\
\hline 0.76000 & 1.038 & 1.030 & 2.634 & 1.024 & 1.01 \\
0.78000 & 2.904 & 2.953 & 2.924 & 2.947 & 2.94 \\
0.81000 & 2.059 & 2.112 & 2.097 & 2.104 & 2.10 \\
0.83000 & 1.675 & 1.721 & 1.693 & 1.714 & 1.71 \\
\hline
\end{tabular}

Table 4.19: Comparison of triplet eigenphase sums. DVR: Present results; C: 18-state variational close-coupling results of Callaway [97]; TB: $R$ matrix propagation method of Taylor and Burke [98]; GB: R matrix propagation method of Geltman and Burke [99]; CW: 11-state variational close-coupling results of Callaway and Wooten [87].

of the Callaway and Wooten calculation. This study is similar to the 18-state calculation of Callaway [97], except that only eleven basis functions are used. These include the exact 1s, 2s, 2p, and 3d states together with seven pseudo-states.

Unfortunately, the eigenphase sums calculated with the present DVR do not seem to give as large eigenphase sums as those calculated with the other methods. As was mentioned previously, the attainment of good convergence and unitarity for the $\mathbf{S}$ matrix in the triplet scattering calculation was more difficult than in the singlet case. This weaker convergence was demonstrated throughout the entire energy range from the $n=2$ to $n=3$ threshold. The most reasonable explanation for this is a need for a larger region of the scattering coordinate being spanned by the $L^{2}$ DVR basis in the triplet case than in the singlet case. This would not be surprising since the symmetry of the triplet spatial wavefunctions of the electrons requires that they not occupy the same regions of space. 


\section{Chapter 5}

\section{Improvements}

One of the most attractive features of discrete variable representations is that the resulting Hamiltonian matrix is very sparse (due to the fact that the potential energy matrix $\mathrm{V}$ is diagonal). This sparsity normally permits the use of special iterative algorithms in the solution of simultaneous equations (such as Lanczos-based algorithms [100,32]) as well as in the finding of eigenvalues [33]. Such algorithms have the tremendous advantage that they do not require the storage of the entire Hamiltonian matrix, but usually only require a user-provided subroutine which performs the multiplication of the Hamiltonian by a column vector input:

$$
\mathbf{y}=\mathbf{H} \cdot \mathbf{x}
$$

Such a subroutine can usually be easily constructed in such a way as to require only a small amount of core memory, far less than is required by complete storage of the Hamiltonian matrix. For example, in this work such a subroutine is used to calculate the bound-free matrix elements in $M_{0}$ of (3.11).

Unfortunately, the rate of convergence (and hence the usefulness) of these iterative algorithms depends heavily upon the condition number of the matrix which is being inverted. The condition number $\kappa$ of a symmetric matrix $\mathbf{A}$ is given by [101] as

$$
\kappa(\mathbf{A})=\|\mathbf{A}\| \cdot\left\|\mathbf{A}^{-1}\right\|
$$


where $\|\mathbf{A}\|$ is the Euclidean norm of the matrix $\mathbf{A}$ given by

$$
\|\mathbf{A}\|=\max \frac{\|\mathbf{A} \mathbf{x}\|}{\|\mathbf{x}\|} \quad \text { for }(\mathbf{x} \neq 0) \text {. }
$$

This can be thought of as the maximum "amplification" ability of the matrix $\mathbf{A}$. This is obviously equal to the largest value of $|\lambda|$ where $\left\{\lambda_{i}\right\}$ is the set of all eigenvalues of A. Therefore the condition number of the matrix $\mathbf{A}$ is

$$
\kappa(\mathbf{A})=\frac{\max |\lambda|}{\min |\lambda|}
$$

Unfortunately, the present formulation of the electron-atom scattering DVR requires the inversion of a matrix with a very large condition number $\kappa$, and this ensures that the convergence of an iterative sparse matrix simultaneous equation solving algorithm is prohibitively slow, much slower than full-storage inversions utilizing a simple $\mathrm{LU}$ decomposition. The large matrix which requires inversion in the $\mathrm{S}$ matrix formulation of the Kohn variational principle is the matrix $\mathbf{H}-\mathbf{E}$. Upon examination of the $L^{2}$ basis, it is not difficult to see why this matrix has a large condition number.

It has already been mentioned that one of the greatest difficulties encountered in trying to develop a DVR for electron-atom scattering is the requirement that a high density of radial points be present near the nucleus in order to adequately represent wavefunctions with a significant amount of S-wave character. The lack of a centrifugal barrier in this partial wave together with the presence of a Coulomb singularity requires the presence of such points.

But because the $L^{2}$ space is composed of a direct product of the two radial spaces with the angular space, these points which are very close to the nucleus are also used to represent wavefunctions with finite angular momentum. Because these points are very close to the nucleus (and hence well-within any centrifugal barrier associated with the non-S-wave scattering), this is equivalent to including very high energy basis functions in the $L^{2}$ basis set. This has the undesirable effect of greatly increasing the largest eigenvalue of the $\mathrm{H}-\mathrm{E}$ matrix, thereby increasing the condition number of the matrix and causing a lack of convergence in any iterative simultaneous equation inversion algorithm. In fact, one witnesses a dramatic increase in the condition number of the $\mathbf{H}-\mathbf{E}$ matrix as one increases the value of the scale parameter 
$\alpha$ defined in (3.29). It will be remembered that this "tuning" parameter determines the density of radial DVR points near the origin.

The usual procedure used to reduce the condition number of the $\mathbf{H}-\mathbf{E}$ matrix is to simply remove those points in the direct product composite space which contribute to including unwanted high energy $L^{2}$ functions in the basis set. This technique is commonly used in current applications of DVR to molecular scattering processes [41], and typically involves establishing a potential energy "cutoff" value $V_{\text {cut }}$. Associated with each composite DVR point there is a value for the potential $V_{i j k l \ldots . . .}$. This point is included in the basis set only if $V_{i j k l \ldots}<V_{c u t}$. The rationale is that the wavefunction is likely to vanish at composite DVR points located in high energy regions of the potential energy surface, and therefore these points need not be included in the $L^{2}$ basis.

Unfortunately, this prescription does not work well in the present study of electron scattering. The composite DVR points most responsible for raising the condition number of the $\mathbf{H}-\mathbf{E}$ matrix are those associated with states in which at least one of the electrons is very close to the nucleus. And while truncating these points from the $L^{2}$ basis would reduce the condition number of the $\mathbf{H}-\mathbf{E}$ matrix, it would also prevent an accurate representation of the most-important $\mathrm{S}$-wave portion of the scattering wavefunction. Since each element of the one-dimensional angular DVR is used to represent both the low and high angular momentum states, it is not possible to eliminate any of these points without compromising accuracy.

But because the number of one-dimensional angular DVR points is quite small compared to the number of points in the radial DVR, it is possible to transform the representation of the angular degree of freedom from a DVR to an FBR (finite basis representation) without sacrificing much of the inherent sparcity of the $\mathbf{H}-\mathbf{E}$ matrix. This is accomplished through the use of the one-dimensional transformation matrix $\mathbf{A}$ defined by (3.60). Since this is an orthogonal matrix, the sparcity of the three-dimensional kinetic energy and overlap matrices is not decreased. The transformation of the potential energy matrix preserves its diagonal character with the respect to the radial DVR indices; only the diagonal character with respect to the angular index is sacrificed. This transformation is tantamount to using the potential- 
optimized DVR in the two radial degrees of freedom only, and a standard Legendre polynomial basis set in the angular degree of freedom.

$$
V_{i j k i^{\prime} j^{\prime} k^{\prime}}=V_{i j k} \delta_{i i^{\prime}} \delta_{j j^{\prime}} \delta_{k k^{\prime}}
$$

is transformed into

$$
V_{i j n i^{\prime} j^{\prime} n^{\prime}}=V_{i j n n^{\prime}} \delta_{i i^{\prime}} \delta_{j j^{\prime}}
$$

Following this transformation, the centrifugal barrier can then be included in the potential energy used in the $V_{\text {cut }}$-based truncation of the $L^{2}$ basis. Then the only radial DVR points located close to the origin which will be included in the $L^{2}$ basis will be those associated with S-wave scattering. This transformation will then keep the condition number $\kappa$ of the $\mathbf{H}-\mathbf{E}$ matrix low enough that an iterative inversion algorithm becomes viable. The calculation of the new $V_{i j n n^{\prime}}$ matrix elements can be performed inexpensively using the original Gauss-Legendre quadrature. Figure 5.1 shows graphically the result of eliminating the angular DVR in favor of an angular FBR.
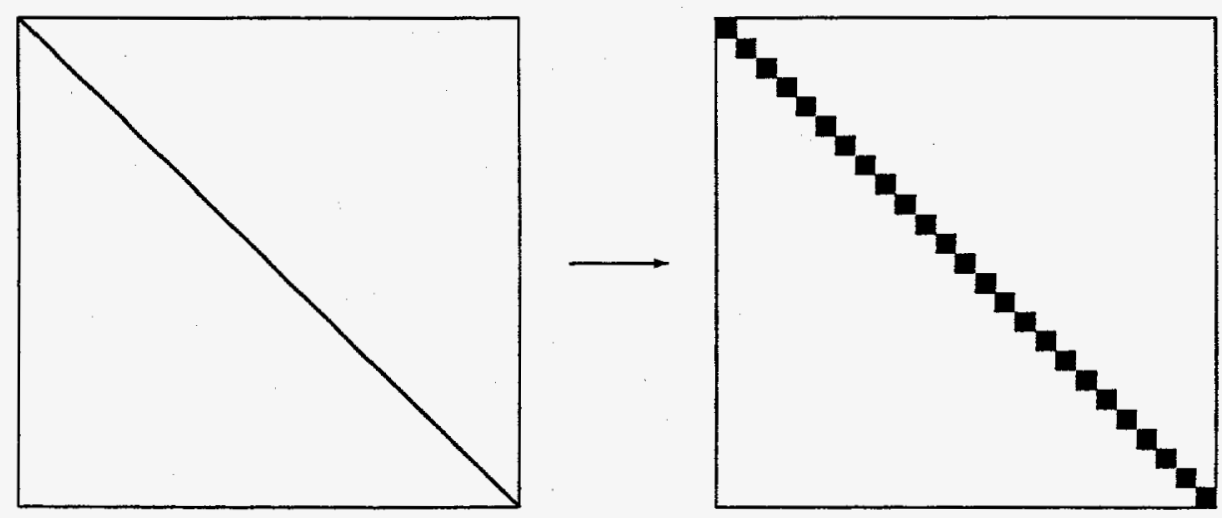

Figure 5.1: Effect on $\mathrm{V}$ of transforming the angular DVR to an FBR. The majority of the sparcity is preserved. The angular index is here assumed to be the fastest varying index.

The substitution of an angular FBR for a DVR would permit calculations requiring expansion of the scattering wavefunction in a much larger number of $L^{2}$ basis functions, since LU decomposition and Gaussian elimination (with their considerable core memory requirement) would no longer be needed to perform the inversion of the 
$\mathbf{H}-\mathbf{E}$ matrix. An alternate technique is presented by Groenenboom and Colbert [43] in which the high energy states associated with DVR points close to the origin in combination with high angular momentum states are projected out of the Krylov space at each iteration of the algorithm. This clearly would have the same effect as the current proposition.

It is clear than the study of an electron scattering from a hydrogen atom does not demonstrate the full power of applying sparse matrix inversion methods to electron-atom scattering. The low core memory requirements of such techniques suggest that more dramatic applications can be found in studying scattering events involving many more degrees of freedom, such as in electron-atom scattering involving multi-electron target atoms. 


\section{Bibliography}

[1] T. Y. Wu and T. Ohmura, Quantum Theory of Scattering (Prentice-Hall, Englewood Cliffs, N.J., 1962).

[2] N. F. Mott and H. S. W. Massey, The Theory of Atomic Collisions, 3rd ed. (Oxford University Press, Oxford, 1965).

[3] R. G. Newton, Scattering Theory of Waves and Particles (McGraw-Hill, New York, 1966).

[4] S. Geltman, Topics in Atomic Collision Theory (Academic Press, New York, 1969).

[5] K. Smith, Calculation of Atomic Collision Processes (Wiley-Interscience, New York, 1971).

[6] C. J. Joachain, Quantum Collision Theory (North-Holland, Amsterdam, 1975).

[7] J. R. Taylor, Scattering Theory (Robert E. Krieger Publishing Company, Malabar, Florida, 1983).

[8] P. G. Burke and M. J. Seaton, Methods Comput. Phys. 10, 1 (1971).

[9] W. A. Lester, in Dynamics of Molecular Collisions, edited by W. H. Miller (Plenum Press, New York, 1976), pp. 1-32.

[10] L. Castillejo, I. C. Percival, and M. J. Seaton, Proc. Roy. Soc. A234, 259 (1960).

[11] P. G. Burke and K. Smith, Rev. Mod. Phys. 34, 458 (1962). 
[12] J. Callaway, Phys. Reports 45, 89 (1978).

[13] R. K. Nesbet, Variational Methods in Electron-Atom Scattering Theory (Plenum Press, New York, 1980).

[14] J. Schwinger, Phys. Rev. 72, 742 (1947).

[15] B. A. Lippmann and J. Schwinger, Phys. Rev. 79, 469 (1950).

[16] M. A. P. Lima et al., Phys. Rev. A 41, 327 (1990).

[17] W. Kohn, Phys. Rev. 74, 1763 (1948).

[18] C. Schwartz, Phys. Rev. 124, 1468 (1961).

[19] E. A. Hylleraas, Z. Phys. 48, 469 (1928).

[20] D. G. Truhlar, J. Abdallah, and R. L. Smith, Adv. Chem. Phys. 25, 211 (1974).

[21] R. K. Nesbet, Adv. At. Mol. Phys. 13, 315 (1977).

[22] C. W. McCurdy and T. N. Rescigno, Phys. Rev. A 39, 4487 (1989).

[23] W. H. Miller and B. M. D. D. J. op de Haar, J. Chem. Phys. 86, 8213 (1987).

[24] J. Z. H. Zhang, S. I. Chu, and W. H. Miller, J. Chem. Phys. 88, 6233 (1988).

[25] D. O. Harris, G. G. Engerholm, and W. D. Gwinn, J. Chem. Phys. 43, 1515 (1965).

[26] A. S. Dickinson and P. R. Certain, J. Chem. Phys. 49, 4209 (1968).

[27] V. I. Krylov, Approximate Calculation of Integrals (Macmillan, New York, 1962).

[28] J. V. Lill, G. A. Parker, and J. C. Light, Chem. Phys. Lett. 89, 483 (1982).

[29] J. C. Light, I. P. Hamilton, and J. V. Lill, J. Chem. Phys. 82, 1400 (1985).

[30] C. Lanczos, J. Res. Natl. Bur. Stand. 45, 255 (1950). 
[31] J. K. Cullum and R. A. Willoughby, Lanczos Algorithms for Large Symmetric Eigenvalue Computations (Birkhä̈ser, Boston, 1985).

[32] C. C. Paige and M. A. Saunders, SIAM J. Numer. Anal. 12, 617 (1975).

[33] B. N. Parlett, The Symmetric Eigenvalue Problem (Prentice-Hall, Englewood Cliffs, N.J., 1980).

[34] J. V. Lill, G. A. Parker, and J. C. Light, J. Chem. Phys. 85, 900 (1986).

[35] S. E. Choi and J. C. Light, J. Chem. Phys. 92, 2129 (1990).

[36] C. Leforestier, J. Chem. Phys. 94, 6388 (1991).

[37] Handbook of Mathematical Functions, edited by M. Abramowitz and I. A. Stegun (National Bureau of Standards Applied Math Series, U. S. GPO, Washington, D.C., 1964).

[38] D. E. Manolopoulos and R. E. Wyatt, Chem. Phys. Lett. 152, 23 (1988).

[39] W. H. Miller, J. Chem. Phys. 50, 407 (1969).

[40] C. Leforestier and W. H. Miller, J. Chem. Phys. 100, 733 (1994).

[41] D. T. Colbert and W. H. Miller, J. Chem. Phys. 96, 1982 (1992).

[42] C. Schwartz, J. Math. Phys. 26, 411 (1985).

[43] G. C. Groenenboom and D. T. Colbert, J. Chem. Phys. 99, 9681 (1993).

[44] T. Seideman and W. H. Miller, J. Chem. Phys. 96, 4412 (1992).

[45] W. H. Thompson and W. H. Miller, Chem. Phys. Lett. 206, 123 (1993).

[46] U. Manthe and W. H. Miller, J. Chem. Phys. 99, 3411 (1993).

[47] U. Manthe, T. Seideman, and W. H. Miller, J. Chem. Phys. 99, 10078 (1993).

[48] R. Kosloff, J. Phys. Chem. 92, 2087 (1988). 
[49] L. F. X. Gaucher and W. H. Miller, Israel J. Chem. 29, 349 (1989).

[50] B. Apagyi, P. Lévay, and K. Ladányi, Phys. Rev. A 37, 4577 (1988).

[51] J. Z. H. Zhang and W. H. Miller, Chem. Phys. Lett. 140, 329 (1987).

[52] J. Z. H. Zhang and W. H. Miller, J. Chem. Phys. 88, 4549 (1988).

[53] J. Z. H. Zhang and W. H. Miller, Chem. Phys. Lett. 153, 465 (1988).

[54] J. Z. H. Zhang and W. H. Miller, J. Chem. Phys. 91, 1528 (1989).

[55] C. W. McCurdy, T. N. Rescigno, and B. I. Schneider, Phys. Rev. A 36, 2061 (1987).

[56] T. N. Rescigno, C. W. McCurdy, and B. I. Schneider, Phys. Rev. A 38, 5921 (1988).

[57] S. D. Parker, C. W. McCurdy, T. N. Rescigno, and B. H. Lengsfield III, Phys. Rev. A 43, 3514 (1991).

[58] J. Schwinger, Phys. Rev. 78, 135 (1950).

[59] M. A. P. Lima, T. L. Gibson, K. Takatsuka, and V. McKoy, Phys. Rev. A 30, $1741(1984)$.

[60] A. J. F. Siegert, Phys. Rev. 56, 750 (1939).

[61] C. Winstead and V. McKoy, Phys. Rev. A 41, 49 (1990).

[62] K. Ladányi, P. Lévay, and B. Apagyi, Phys. Rev. A 38, 3365 (1988).

[63] D. Baye and P.-H. Heenen, J. Phys. A 19, 2041 (1986).

[64] M. Vincke, L. Malegat, and D. Baye, J. Phys. B 26, 811 (1993).

[65] J. Botero and J. Shertzer, Phys. Rev. A 46, R1155 (1992).

[66] K. J. Bathe, Finite Element Procedures in Engineering Analysis (Prentice-Hall, Englewood Cliffs, N.J., 1982). 
[67] G. Strang and G. J. Fix, An Analysis of the Finite Element Method (PrenticeHall, Englewood Cliffs, N.J., 1973).

[68] R. Poet, J. Phys. B 13, 2995 (1980).

[69] Y. D. Wang and J. Callaway, Phys. Rev. A 48, 2058 (1993).

[70] C. L. Pekeris, Phys. Rev. 126, 1470 (1962).

[71] M. Rotenberg, Anṇ. Phys. (N.Y.) 19, 262 (1962).

[72] H. Shull and P.-O. Löwdin, J. Chem. Phys. 30, 617 (1959).

[73] G. R. Taylor and R. G. Parr, Proc. Natl. Acad. Sci. U.S. 38, 154 (1952).

[74] E. Yurtsever, O. Yilmaz, and D. D. Shillady, Chem. Phys. Lett. 85, 111 (1982).

[75] M. Rotenberg, Advan. At. Mol. Phys. 6, 233 (1970).

[76] P. M. Morse and W. P. Allis, Phys. Rev. 44, 269 (1933).

[77] J. Echave and D. C. Clary, Chem. Phys. Lett. 190, 225 (1992).

[78] Table of Integrals, Series, and Products, edited by I. S. Gradshteyn and I. M. Ryzhik (Harcourt Brace Jovanovich, Academic Press, Inc., New York, 1980).

[79] G. Arfken, Mathematical Methods for Physicists, 3rd ed. (Harcourt Brace Jovanovich, Academic Press, Inc., New York, 1985).

[80] S. Kanfer and M. Shapiro, J. Phys. Chem. 88, 3964 (1984).

[81] J. J. Sakurai, Modern Quantum Mechanics (Benjamin/Cummings Publishing Co., Menlo Park, CA, 1985).

[82] F. A. Cotton, Chemical Applications of Group Theory, 2nd ed. (WileyInterscience, New York, 1971).

[83] R. D. Levine and R. B. Bernstein, Molecular Reaction Dynamics and Chemical Reactivity (Oxford University Press, New York, 1987). 
[84] M. Gailitis and R. Damburg, Proc. Phys. Soc. 82, 192 (1963).

[85] M. E. Rose, Elementary Theory of Angular Momentum (John Wiley and Sons, Inc., New York, 1957).

[86] G. J. Seiler, R. S. Oberoi, and J. Callaway, Phys. Rev. A 3, 2006 (1971).

[87] J. Callaway and J. W. Wooten, Phys. Rev. A 9, 1924 (1973).

[88] R. S. Oberoi and R. K. Nesbet, J. Comp. Phys. 12, 526 (1973).

[89] P. G. Burke and H. M. Schey, Phys. Rev. 126, 147 (1962).

[90] B. R. Johnson, J. Chem. Phys. 69, 4678 (1978).

[91] B. R. Johnson, National Resource for Computation in Chemisty, University of California, Berkeley, NRCC, Proceedings No. 5, p.86 (1979).

[92] L. M. Hubbard, S.-H. Shi, and W. H. Miller, J. Chem. Phys. 78, 2381 (1983).

[93] G. C. Groenenboom, (private communication).

[94] T. Seideman and W. H. Miller, J. Chem. Phys. 97, 2499 (1992).

[95] H. W. Jang and J. C. Light, J. Chem. Phys. 99, 1057 (1993).

[96] T. N. Rescigno and B. I. Schneider, Phys. Rev. A 37, 1044 (1988).

[97] J. Callaway, Phys. Rev. A 26, 199 (1982).

[98] A. J. Taylor and P. G. Burke, Proc. Phys. Soc. 92, 366 (1967).

[99] S. Geltman and P. G. Burke, J. Phys. B 3, 1062 (1970).

[100] B. N. Parlett, Lin. Alg. Appl. 34, 269 (1980).

[101] G. Strang, Linear Algebra and Its Applications (Academic Press, Orlando, Florida, 1980). 